\begin{abstract}
Title of Document:

EXPERIMENTAL DETECTION AND QUANTITATIVE INTERROGATION OF DAMAGE IN A JOINTED COMPOSITE STRUCTURE

Vanessa J. Gentzen, Master of Science, 2008

Directed By:

Professor Norman Wereley, Department of Aerospace Engineering
\end{abstract}

The aerospace field has concentrated substantial attention toward the development of structural health monitoring (SHM) systems for multidisciplinary applications. Research is motivated by catastrophic failures of operational systems which may have been avoided with the prior implementation of a successful damage prediction method. This research utilizes a smart sensor array to collect sensing information over a variety of damaged scenarios on a composite lap-joint assembly. Damage was implemented as bolt torque loss within the joint. A damage index was used as the key diagnostic feature to interrogate damage within the structure. Pattern recognition of the damage index, in addition to a rule-based, statistical discrimination method was employed to detect, localize and quantify damage due to bolt torque loss in the structure. The methodology accurately detected the presence of damage within the joint, localized the damage within the structures four quadrants, and assessed the level of torque loss. 


\title{
EXPERIMENTAL DETECTION AND QUANTITATIVE INTERROGATION OF DAMAGE IN A JOINTED COMPOSITE STRUCTURE
}

\author{
By \\ Vanessa J. Gentzen \\ Thesis submitted to the Faculty of the Graduate School of the \\ University of Maryland, College Park, in partial fulfillment \\ of the requirements for the degree of \\ Master of Science \\ 2008
}

Advisory Committee:

Professor Norman Wereley, Chair/Advisor

Professor Darryll Pines

Professor Sung Lee 


\section{Acknowledgements}

I would like to sincerely thank my advisor, Professor Norman Wereley for not only providing me the opportunity to explore an exciting and challenging area of research, but for consistently believing in my abilities even when I found myself in doubt. Also, thanks to Dr. Young-Tai Choi for his continual guidance and enthusiasm in the research lab. In particular, I must thank Dr. Mary Bowden and Dr. James Hubbard for providing me the first sparks of inspiration upon entering College Park. I am monumentally grateful to my advisory committee and to the entire faculty of the University of Maryland's Department of Aerospace Engineering; my brief time in the department has been more rewarding than I could have anticipated.

To my fellow graduate students, you provided a sense of community to a new face on campus and for that, I am deeply grateful. I have learned a great deal from you as colleagues and friends.

I would be remiss not to extend my deep appreciation to my family and friends for their abiding patience, love and support. In particular, I'd like to thank my dear friend Gregory Miller for keeping me positive throughout my graduate experience.

Most importantly, I acknowledge the strength and support that is continually provided to me by God. For that, I am the most thankful and forever will be. 


\section{Table of Contents}

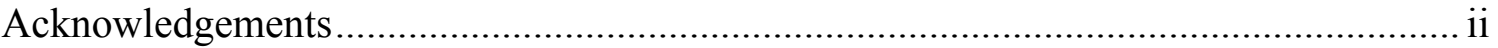

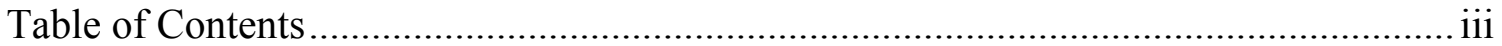

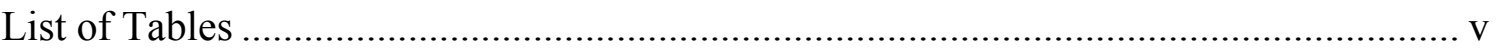

List of Figures .................................................................................................. vi

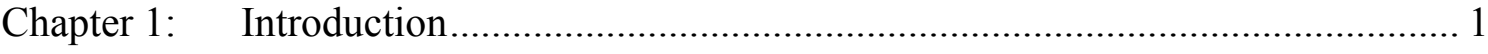

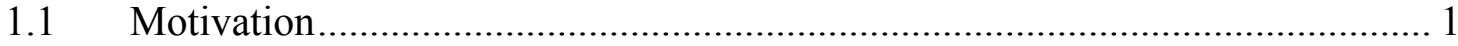

1.2 Overview of the Structural Health Monitoring Process...................................... 4

$1.3 \quad$ Previous Work and Fundamental Theory …………............................................ 7

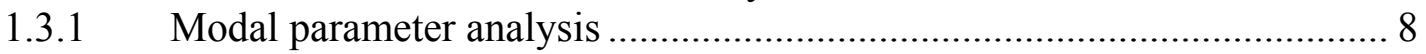

1.3.2 Acousto-Ultrasonics..................................................................... 9

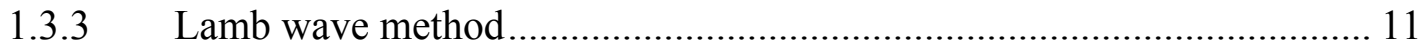

1.3.4 Signal processing using wavelet transformation....................................... 12

1.3.5 Interrogation of bolted composite structures ............................................ 13

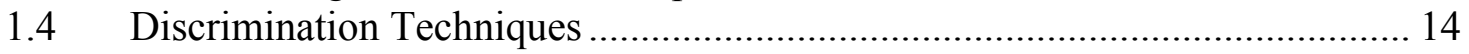

$1.5 \quad$ Research Aim......................................................................................... 15

Chapter 2: Experimental Procedure.................................................................... 17

2.1 Jointed Composite Panel......................................................................... 17

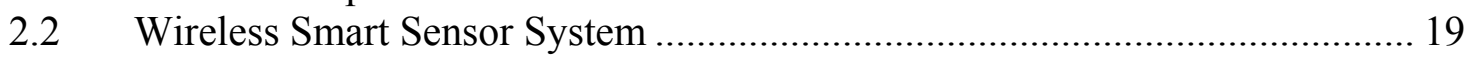

2.3 Signal Noise Filtration and Running Average Method...................................... 22

2.4 Damage Due to Bolt Torque Loss .............................................................. 23

$2.5 \quad$ Data Compression: Damage Index ……………....................................... 24

2.6 Baseline Sensing Variation Over Time.............................................................. 27

2.7 Baseline Sensing Variation due to Temperature Increase …………………... 28

Chapter 3: Damage Due to Bolt Torque Loss ………………………………........... 30

$3.1 \quad$ Bolt 0 damage due to torque loss............................................................. 30

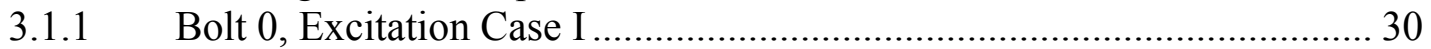

3.1.2 Bolt 0, Excitation Case II...................................................................... 39

3.1.3 Bolt 0, Excitation Case III .................................................................... 48

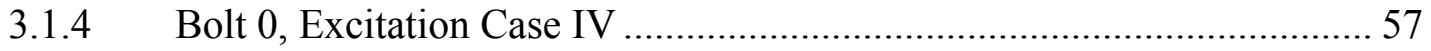

3.1.5 Comparison of Bolt 0 excitation configurations ......................................... 66

3.1.6 Detection of smaller torque loss percentages............................................ 70

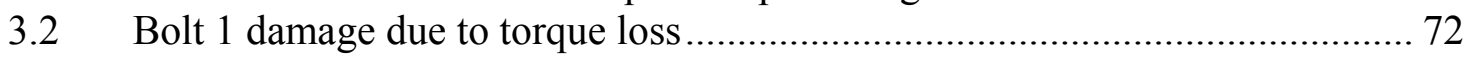

3.2.1 Bolt 1, Excitation Case I ................................................................... 72

3.2.2 Bolt 1, Excitation Case II...................................................................... 77

3.2.3 Bolt 1, Excitation Case III ……………………................................ 81

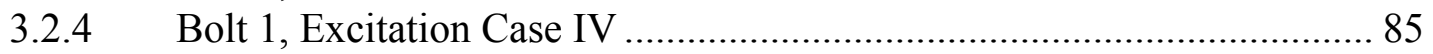

3.2.5 Comparison of Bolt 1 excitation configurations....................................... 89

3.3 Bolt 2 damage due to torque loss............................................................... 92

3.3.1 Bolt 2, Excitation Case I .................................................................... 93

3.3.2 Bolt 2, Excitation Case II.................................................................... 97

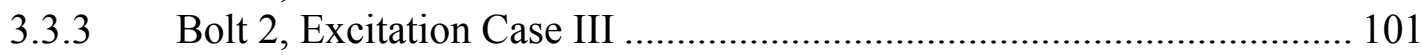

3.3.4 Bolt 2, Excitation Case IV ...................................................................... 105

3.3.5 Comparison of Bolt 2 excitation configurations..................................... 109 


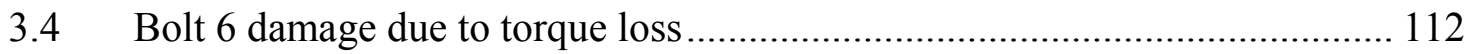

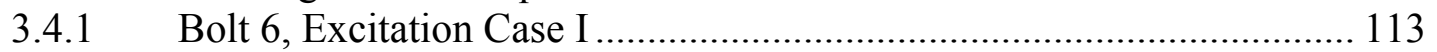

3.4.2 Bolt 6, Excitation Case II................................................................. 116

3.4.3 Bolt 6, Excitation Case III ................................................................. 119

3.4.4 Bolt 6, Excitation Case IV ................................................................ 122

3.4.5 Comparison of Bolt 6 excitation configurations.................................... 125

3.5 Dual-bolt damage due to torque loss ..................................................... 129

3.5.1 Bolt 0 and Bolt 1, Excitation Case I …….......................................... 130

3.5.2 Bolt 0 and Bolt 1, Excitation Case II .................................................. 133

3.5.3 Bolt 0 and Bolt 1, Excitation Case III................................................... 136

3.5.4 Bolt 0 and Bolt 1, Excitation Case IV................................................... 139

3.5.5 Comparison of dual Bolt 0 and Bolt 1 excitation configurations ........... 142

3.5.6 Bolt 0 and Bolt 6, Excitation Case I .................................................... 145

3.5.7 Bolt 0 and Bolt 6, Excitation Case II .................................................. 149

3.5.8 Bolt 0 and Bolt 6, Excitation Case III.............................................. 152

3.5.9 Bolt 0 and Bolt 6, Excitation Case IV.................................................. 155

3.5.10 Comparison of dual Bolt 0 and Bolt 6 excitation configurations ............ 158

3.6 Sensing Variation of Undamaged Composite Jointed Panel over Time......... 162

3.7 Sensing Variation due to Temperature Increase of an Active Sensor ............ 168

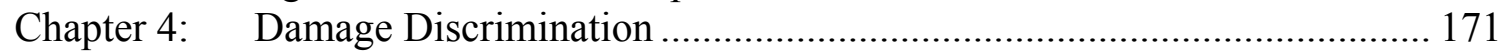

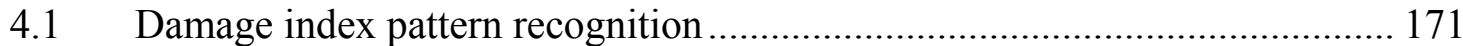

4.2 Level 2: Damage localization algorithm................................................... 174

4.3 Level 3: Damage quantification algorithm ................................................. 180

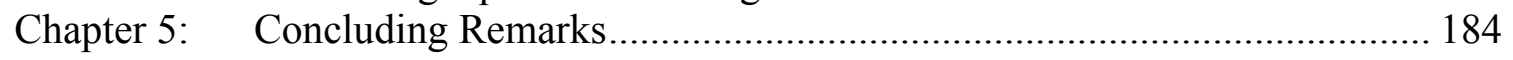

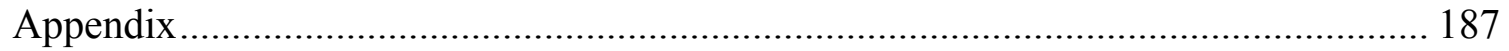

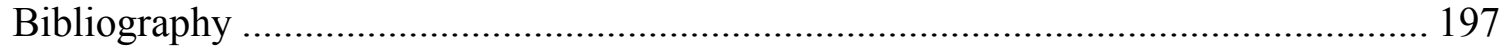




\section{List of Tables}

Table 4-1-1: DI grid, 100\% B0 torque loss .................................... 170

Table 4-1-2: $D I$ grid, 75\% B1 torque loss ......................................... 172

Table 4-1-3: DI grid, 25\% B2 torque loss .................................... 172

Table 4-1-4: $D I_{\text {mag }}$; B0 100\% torque loss, B1 75\% torque loss, B2 25\% torque loss ....173

Table 4-1-5: Validation experimental run matrix 


\section{List of Figures}

Figure 1-1-1: Fuselage damage to Aloha Airlines Flight 243, April 1988 ....................... 2

Figure 1-1-2: Military aircraft graveyard............................................................... 3

Figure 1-2-1. Flowchart, implementation of a structural health monitoring program [8] .. 5

Figure 1-3-1. Acousto-Ultrasonic schematic within a structure. ……………………...... 10

Figure 2-1-1: The jointed composite panel............................................................... 18

Figure 2-2-1: The jointed composite panel with piezoelectric patch assembly............... 19

Figure 2-2-2: Formula excitation output signal with $20 \mathrm{kHz}$ excitation frequency.......... 20

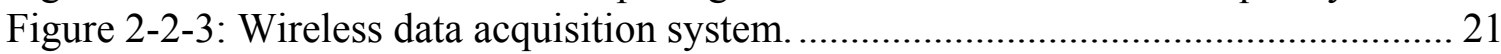

Figure 2-2-4: Specimen quadrants and configuration Cases I, II, III, IV......................... 22

Figure 2-7-1: Baseline temperature variation of S1, E2 excitation. ................................. 29

Figure 3-1-1. Excitation Case I, Bolt 0 torque loss........................................................ 30

Figure 3-1-2. Sensing time response, B0 torque loss, E1 $40 \mathrm{kHz}$ excitation.................... 31

Figure 3-1-3. Scattering signals, B0 torque loss, E1 $40 \mathrm{kHz}$ excitation. .......................... 33

Figure 3-1-4. Normalized scattering signals, B0 torque loss, E1 $40 \mathrm{kHz}$ excitation........ 34

Figure 3-1-5. Damage index using the energy magnitudes in frequency vs. B0 torque loss

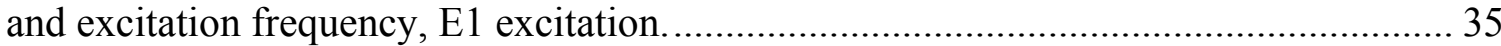

Figure 3-1-6. Damage index using the signals in time vs. B0 torque loss and excitation

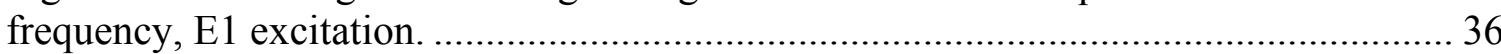

Figure 3-1-7. Average damage index, B0 torque loss, E1 excitation. ............................. 38

Figure 3-1-8. Total damage index, $\mathrm{B} 0$ torque loss, E1 excitation. ..................................... 39

Figure 3-1-9. Excitation Case II, variable Bolt 0 torque loss. .......................................... 40

Figure 3-1-10. Sensing time response, B0 torque loss, E2 $40 \mathrm{kHz}$ excitation................... 41

Figure 3-1-11. Scattering signals, B0 torque loss, E2 $40 \mathrm{kHz}$ excitation........................ 42

Figure 3-1-12. Normalized Scattering signals, B0 torque loss, E2 $40 \mathrm{kHz}$ excitation. .... 43

Figure 3-1-13. Damage index using the energy magnitudes in frequency vs. $\mathrm{B} 0$ torque loss and excitation frequency, E2 excitation.

Figure 3-1-14. Damage index using signals in time in vs. B0 torque loss and excitation

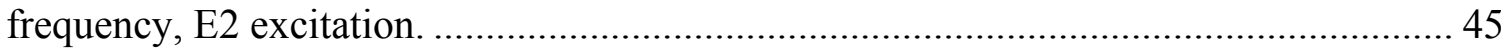

Figure 3-1-15. Average damage index, B0 torque loss, E2 excitation. ........................... 47

Figure 3-1-16. Total damage index, B0 torque loss, E2 excitation. .................................48

Figure 3-1-17. Excitation Case III, variable Bolt 0 torque loss........................................ 49

Figure 3-1-18. Sensing time response, B0 torque loss, E3 $40 \mathrm{kHz}$ excitation.................. 50

Figure 3-1-19. Scattering signals, B0 torque loss, E3 $40 \mathrm{kHz}$ excitation. ........................ 51

Figure 3-1-20. Normalized scattering signals, B0 torque loss, E3 $40 \mathrm{kHz}$ excitation...... 52

Figure 3-1-21. Damage index using the energy magnitudes in frequency vs. B0 torque loss and excitation frequency, E3 excitation.

Figure 3-1-22. Damage index using the signals in time vs. B0 torque loss and excitation

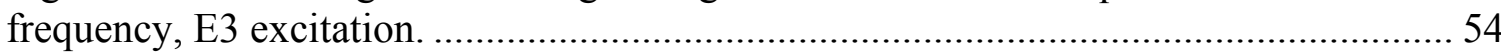

Figure 3-1-23. Average damage index, B0 torque loss, E3 excitation. ........................... 56

Figure 3-1-24. Total damage index, B0 torque loss, E3 excitation. ................................ 57

Figure 3-1-25. Excitation Case IV, variable Bolt 0 torque loss........................................ 58

Figure 3-1-26. Sensing time response, B0 torque loss, E4 $40 \mathrm{kHz}$ excitation...................59

Figure 3-1-27. Scattering signals, B0 torque loss, E4 $40 \mathrm{kHz}$ excitation......................... 60 
Figure 3-1-28. Normalized scattering signals, B0 torque loss, E4 $40 \mathrm{kHz}$ excitation...... 61 Figure 3-1-29. Damage index using the energy magnitudes in frequency vs. B0 torque loss and excitation frequency, E4 excitation.

Figure 3-1-30. Damage index using the signals in time vs. B0 torque loss and excitation

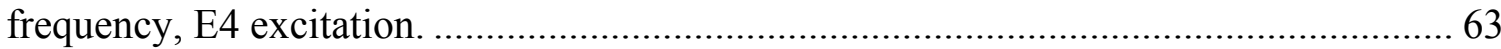

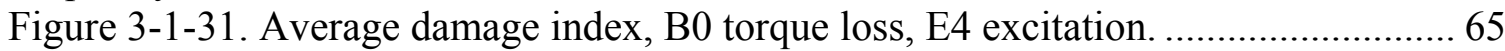

Figure 3-1-32. Total damage index, B0 torque loss, E4 excitation. ............................... 66

Figure 3-1-33. Comparison of normalized average damage index, B0 torque loss......... 68

Figure 3-1-34. Comparison of normalized average damage indices, B0 torque loss; $D I_{E 2, S 3}$

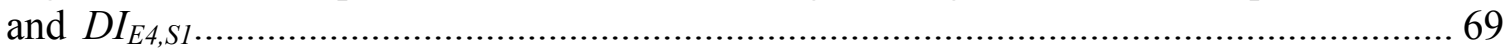

Figure 3-1-35. Estimated damage area due to B0 torque loss. .................................... 70

Figure 3-1-36. Normalized average damage index due to smaller B0 torque loss.......... 71

Figure 3-1-37. Total damage index due to smaller B0 torque loss............................. 72

Figure 3-2-1. Excitation Case I, variable Bolt 1 torque loss......................................... 73

Figure 3-2-2. Scattering signals, B1 torque loss, E1 $40 \mathrm{kHz}$ excitation......................... 74

Figure 3-2-3. Damage index using the energy magnitudes in frequency vs. B1 torque loss

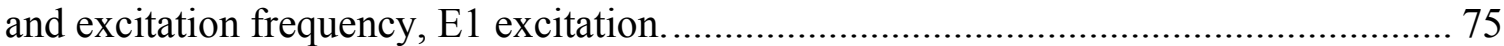

Figure 3-2-4. Damage index using the signals in time vs. B1 torque loss and excitation

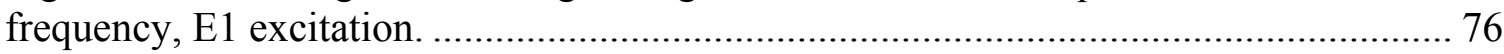

Figure 3-2-5. Excitation Case II, variable Bolt 1 torque loss. ...................................... 77

Figure 3-2-6. Scattering signals, B1 torque loss, E2 $40 \mathrm{kHz}$ excitation. ......................... 78

Figure 3-2-7. Damage index using the energy magnitudes in frequency vs. B1 torque loss and excitation frequency, E2 excitation................................................................ 79

Figure 3-2-8. Damage index using the signals in time vs. B1 torque loss and excitation

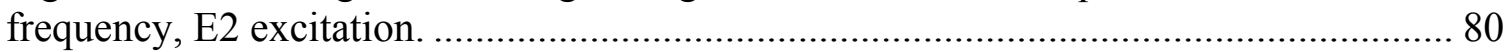

Figure 3-2-9. Excitation Case III, variable Bolt 1 torque loss..................................... 81

Figure 3-2-10. Scattering signals, B1 torque loss, E3 $40 \mathrm{kHz}$ excitation........................ 82

Figure 3-2-11. Damage index using the energy magnitudes in frequency vs. B1 torque

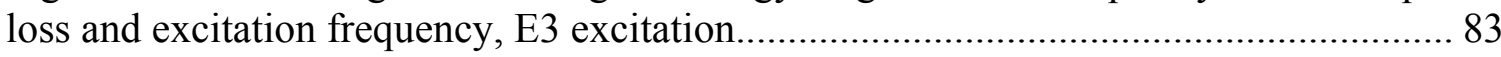

Figure 3-2-12. Damage index using the energy magnitudes in frequency vs. B1 torque

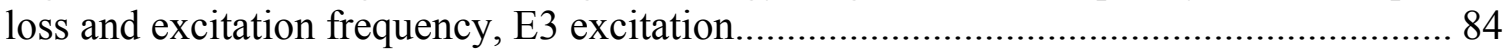

Figure 3-2-13. Excitation Case IV, variable Bolt 1 torque loss................................... 85

Figure 3-2-14. Scattering signals, B1 torque loss, E4 $40 \mathrm{kHz}$ excitation........................... 86

Figure 3-2-15. Damage index using the energy magnitudes in frequency vs. B1 torque

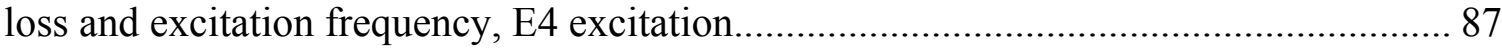

Figure 3-2-16. Damage index using the energy magnitudes in frequency vs. B1 torque

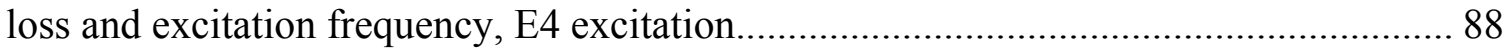

Figure 3-2-17. Comparison of normalized average damage index, B1 torque loss......... 90

Figure 3-2-18. Comparison of normalized average damage indices, B1 torque loss; $D I_{E 4, S I}$

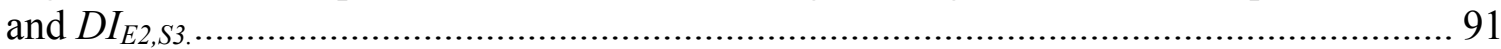

Figure 3-2-19. Estimated damage area due to B1 torque loss. .................................... 92

Figure 3-3-1. Excitation Case I, variable Bolt 2 torque loss....................................... 93

Figure 3-3-2. Scattering signals, B2 torque loss, E1 $40 \mathrm{kHz}$ excitation. ........................ 94

Figure 3-3-3. Damage index using the energy magnitudes in frequency vs. B2 torque loss

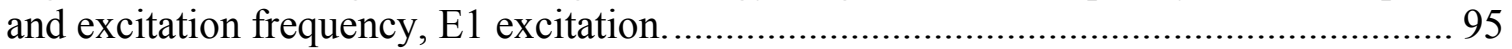


Figure 3-3-4. Damage index using the energy magnitudes in frequency vs. B2 torque loss

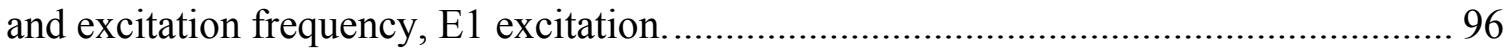
Figure 3-3-5. Excitation Case II, variable Bolt 2 torque loss. .......................................... 97 Figure 3-3-6. Scattering signals, B2 torque loss, E2 $40 \mathrm{kHz}$ excitation frequency.......... 98 Figure 3-3-7. Damage index using the energy magnitudes in frequency vs. B2 torque loss and excitation frequency, E2 excitation.

Figure 3-3-8. Damage index using the signals in time vs. B2 torque loss and excitation

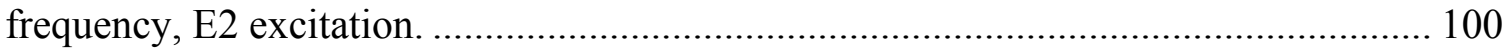
Figure 3-3-9. Excitation Case III, variable Bolt 2 torque loss........................................ 101 Figure 3-3-10. Scattering signals, B2 torque loss, E3 $40 \mathrm{kHz}$ excitation...................... 102 Figure 3-3-11. Damage index using the energy magnitudes in frequency vs. B2 torque loss and excitation frequency, E3 excitation................................................................. 103 Figure 3-3-12. Damage index using the energy signals in time vs. B2 torque loss and

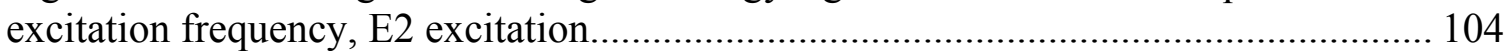
Figure 3-3-13. Excitation Case IV, variable Bolt 2 torque loss.................................... 105 Figure 3-3-14. Scattering signals, B2 torque loss, E4 $40 \mathrm{kHz}$ excitation frequency...... 106 Figure 3-3-15. Damage index using the energy magnitudes in frequency vs. B2 torque

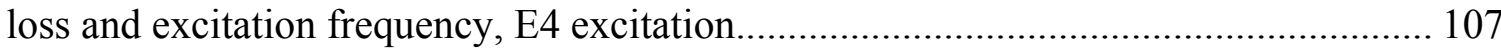
Figure 3-3-16. Damage index using the signals in time vs. B2 torque loss and excitation

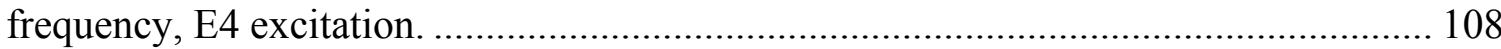
Figure 3-3-17. Comparison of normalized average damage index, B2 torque loss........ 110 Figure 3-3-18. Comparison of normalized average damage indices, B2 torque loss; $D I_{E 2, S 3}$

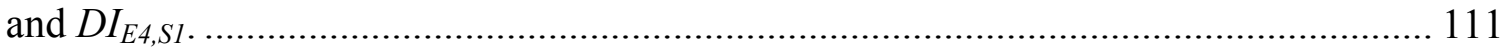
Figure 3-3-19. Estimated damage area due to B2 torque loss. ...................................... 112 Figure 3-4-1. Excitation Case I, variable Bolt 6 torque loss.......................................... 113 Figure 3-4-2. Damage index using the energy magnitudes in frequency vs. B6 torque loss

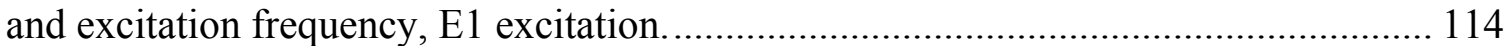
Figure 3-4-3. Damage index using the signals in time vs. B6 torque loss and excitation

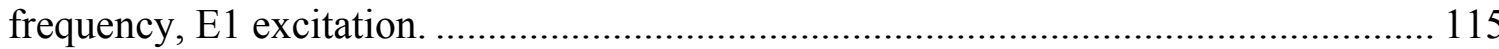
Figure 3-4-4. Excitation Case II, variable Bolt 6 torque loss. ....................................... 116 Figure 3-4-5. Damage index using the energy magnitudes in frequency vs. B6 torque loss and excitation frequency, E2 excitation................................................................ 117 Figure 3-4-6. Damage index using the signals in time vs. B6 torque loss and excitation

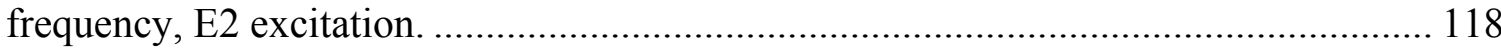
Figure 3-4-7. Excitation Case III, variable Bolt 6 torque loss....................................... 119 Figure 3-4-8. Damage index using the energy magnitudes in frequency vs. B6 torque loss and excitation frequency, E3 excitation...................................................................... 120 Figure 3-4-9. Damage index using the signals in time vs. B6 torque loss and excitation

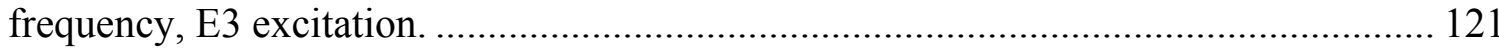

Figure 3-4-10. Excitation Case IV, variable Bolt 6 torque loss...................................... 122 Figure 3-4-11. Damage index using the energy magnitudes in frequency vs. B6 torque loss and excitation frequency, E4 excitation................................................................... 123 Figure 3-4-12. Damage index using the signals in time vs. B6 torque loss and excitation frequency, E4 excitation. ..................................................................................... 124 Figure 3-4-13. Comparison of normalized average damage index, B6 torque loss........ 126 
Figure 3-4-14. Comparison of normalized average damage indices, B6 torque loss; $D I_{E 3, S 2}$

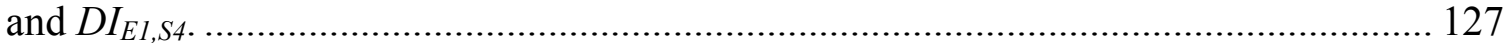
Figure 3-4-15. Comparison of normalized average damage indices, B6 torque loss; $D I_{E 1, S 2}$

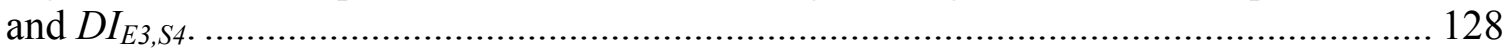
Figure 3-4-16. Estimated damage area due to B6 torque loss. ................................... 129 Figure 3-5-1. Excitation Case I, dual Bolt 0 and Bolt 1 variable torque loss................ 130 Figure 3-5-2. Damage index using the energy magnitudes in frequency vs. B0 and B1 torque loss and excitation frequency, E1 excitation.

Figure 3-5-3. Damage index using the signals in time vs. B0 and B1 torque loss and

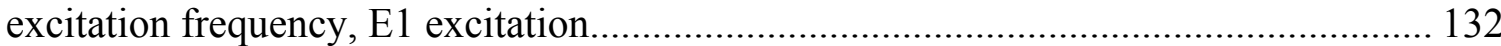
Figure 3-5-4. Excitation Case II, dual Bolt 0 and Bolt 1 variable torque loss............... 133 Figure 3-5-5. Damage index using the energy magnitudes in frequency vs. B0 and B1 torque loss and excitation frequency, E2 excitation.

Figure 3-5-6. Damage index using the signals in time vs. B0 and B1 torque loss and

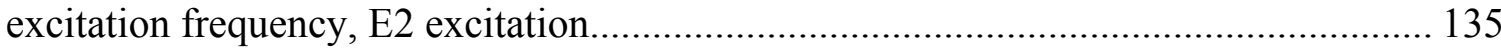

Figure 3-5-7. Excitation Case III, dual Bolt 0 and Bolt 1 variable torque loss. ............ 136 Figure 3-5-8. Damage index using the energy magnitudes in frequency vs. B0 and B1 torque loss and excitation frequency, E3 excitation.

Figure 3-5-9. Damage index using the signals in time vs. B0 and B1 torque loss and

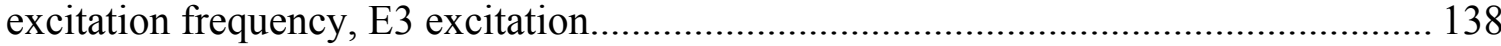

Figure 3-5-10. Excitation Case IV, dual Bolt 0 and Bolt 1 variable torque loss........... 139 Figure 3-5-11. Damage index using the energy magnitudes in frequency vs. B0 and B1 torque loss and excitation frequency, E4 excitation. .............................................. 140 Figure 3-5-12. Damage index using the signals in time vs. B0 and B1 torque loss and excitation frequency, E4 excitation.....

Figure 3-5-13. Comparison of normalized average damage index, Bolt 0 and Bolt 1 dualbolt torque loss.

Figure 3-5-14. Comparison of normalized average damage indices, Bolt 0 and Bolt 1

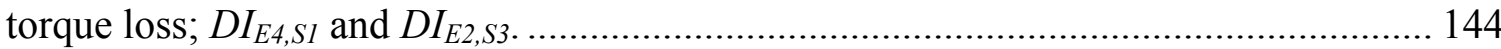

Figure 3-5-15. Estimated damage area due to Bolt 0 and Bolt 1 torque loss. ............... 145

Figure 3-5-16. Excitation Case I, dual Bolt 0 and Bolt 6 variable torque loss............. 146 Figure 3-5-17. Damage index using the energy magnitudes in frequency vs. B0 and B6 torque loss and excitation frequency, E1 excitation.

Figure 3-5-18. Damage index using the signals in time vs. B0 and B6 torque loss and

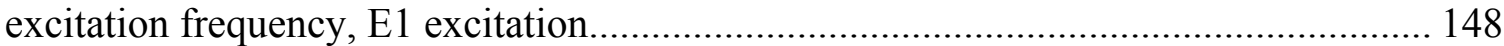
Figure 3-5-19. Excitation Case II, dual Bolt 0 and Bolt 6 variable torque loss............ 149 Figure 3-5-20. Damage index using the energy magnitudes in frequency vs. B0 and B6 torque loss and excitation frequency, E2 excitation.

Figure 3-5-21. Damage index using the signals in time vs. B0 and B6 torque loss and excitation frequency, E2 excitation.

Figure 3-5-22. Excitation Case III, dual Bolt 0 and Bolt 6 variable torque loss. .......... 152 Figure 3-5-23. Damage index using the energy magnitudes in frequency vs. B0 and B6 torque loss and excitation frequency, E3 excitation.

Figure 3-5-24. Damage index using the signals in time vs. B0 and B6 torque loss and excitation frequency, E3 excitation. 
Figure 3-5-26. Damage index using the energy magnitudes in frequency vs. B0 and B6 torque loss and excitation frequency, E4 excitation.

Figure 3-5-27. Damage index using the signals in time vs. B0 and B6 torque loss and excitation frequency, E4 excitation.

Figure 3-5-29. Comparison of normalized average damage indices, Bolt 0 and Bolt 6 torque loss; $D I_{E 4, S 1}$ and $D I_{E 2, S 3}$.

Figure 3-5-30. Comparison of normalized average damage indices, Bolt 0 and Bolt 6 torque loss; $D I_{E 3, S 2}$ and $D I_{E 1, S 4}$. 161

Figure 3-5-31. Estimated damage area due to Bolt 0 and Bolt 1 torque loss. ................ 162 Figure 3-6-1. Normalized baseline time response over five months, E2 $40 \mathrm{kHz}$ excitation. 164

Figure 3-6-2. Normalized baseline voltage response vs. E2 excitation frequency over five

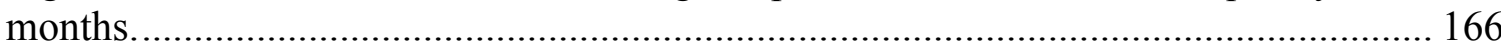

Figure 3-6-3. Average baseline time response, E2 $40 \mathrm{kHz}$ excitation.......................... 168

Figure 3-7-1. S1 maximum time response vs. temperature, E2 excitation..................... 169

Figure 3-7-2. S1 normalized time response vs. temperature, E2 excitation. …………... 170

Figure 4-1-1. Path of straight-across excitation....................................................... 175

Figure 4-1-2. Path of diagonally-across excitation on the composite jointed panel....... 177

Figure 4-1-3. Quadrant 1 vs. Quadrant 3 damage index sensing information................. 180

Figure 4-1-4. Quadrant 1 vs. Quadrant 3 damage with damage threshold bands........... 181

Figure 4-1-5. Quadrant 1 vs. Quadrant 3 discriminator validation run results. .............. 183 


\section{Chapter 1: Introduction}

\subsection{Motivation}

The ability to continually monitor an engineering structure has been the motivated a great deal of research in the structural health monitoring (SHM) arena. Research contributions across multi-disciplinary engineering fields have proven functional in various experimental and operational applications. In particular, the aerospace field has concentrated substantial attention toward the development of SHM systems for applications in space, rotorcraft, commercial and military aerospace structures.

Precipitous advances in engineering and technology demand that SHM methods increase their adaptability to include more complex structural applications. Massive, multifaceted structures have prompted the need for global damage detection methods. However, technological advances are not the sole motivation behind the demand for more concentrated SHM efforts. Federal funding of large-scale defense and civilian contracted engineering programs have gained the attention of tax-paying citizens and prompted ample media coverage. Spectacular failures can involve a colossal monetary loss, and more significantly, the loss of human life in the most tragic instances. Malfunction in the public eye results in exceeding concern and a new outcry for additional preventative research and maintenance advances. The public voice prompts political interest which provokes industry and regulatory agencies to re-direct spending toward the advancement of structural safety programs.

One incident in particular, the April 1988 in-flight fuselage detachment of Aloha Airlines Flight 243 resulted in the death of one flight attendant. Figure 1-1-1 shows the 
result of this structural failure. A pre-flight visual inspection according to Federal Aviation Administration (FAA) standards concluded operable flight conditions prior to take-off. Considerable media coverage of this incident triggered the prioritization of SHM development among the civilian aircraft community.

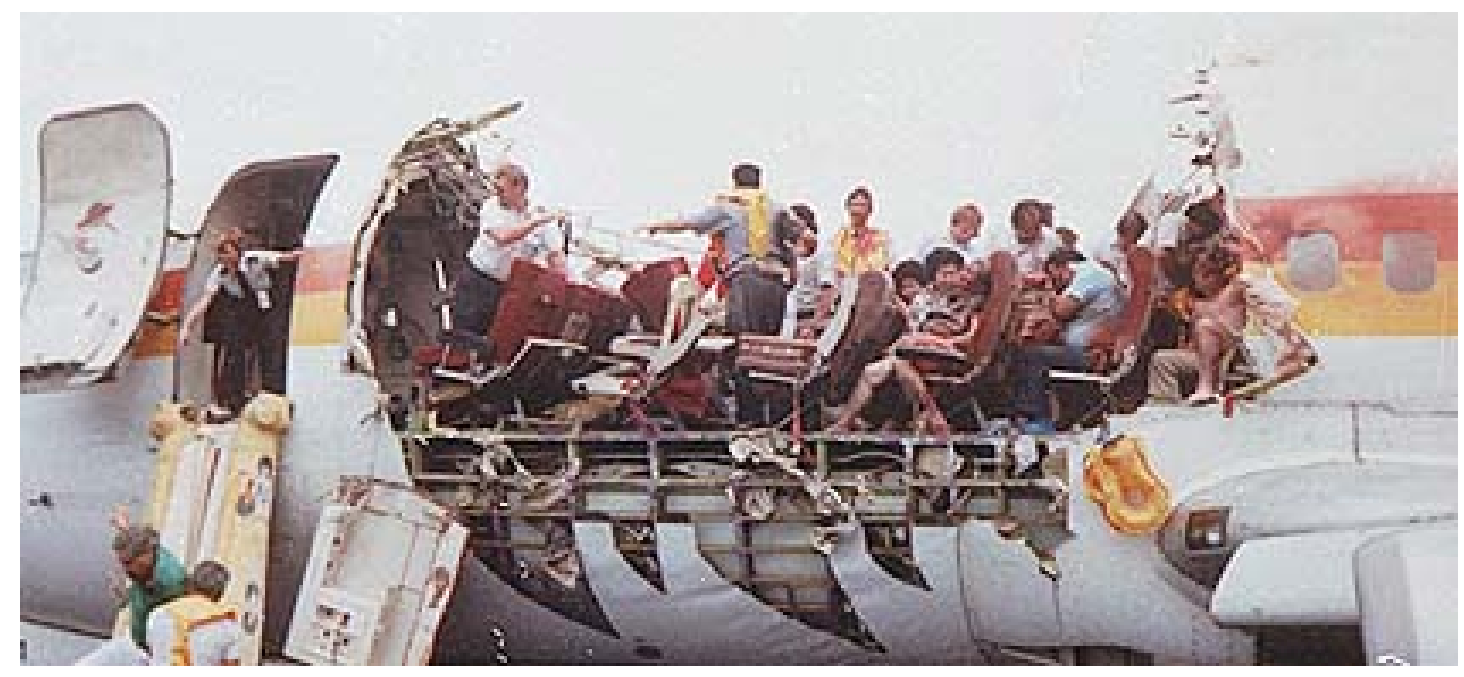

Figure 1-1-1: Fuselage damage to Aloha Airlines Flight 243, April 1988.

The spacecraft industry is another which has experienced heavy public scrutiny. Due to a thermal protection system failure on the leading edge of the ill-fated Space Shuttle Columbia, the vehicle suffered a catastrophic failure upon re-entry, resulting in the death of seven National Aeronautics and Space Administration (NASA) astronauts. This tragedy prompted a painstakingly detailed investigation of the structural basis for failure and brought light to a critical need for NASA's implementation of a reliable health monitoring system [1]. The development of a Shuttle Modal Inspection System (SMIS) addresses the difficulties of accessing and identifying structural damage underneath the shuttle's thermal protection system [2]. 
As the aerospace field continues to make advancements in aircraft and structural designs, the aging systems of the past are approaching the end of their predicted lifetimes. System maintenance becomes a prominent and costly topic for concern. Figure 1-1-2 shows the popular airplane graveyard at Davis-Monthan Air Force Base in Tucson, Arizona. The picture shows a fraction of the large number of military aircraft that are decommissioned due to exceeded lifetime, irreparable damage, or out-dated technology. While it is not always possible to prolong the lifetime of an aging aerospace structure, advancements in SHM and a similar program for Condition Based Maintenance (CBM) may extend the serviceability of these vehicles which are not easily or inexpensively replaced. A successful CBM system will assess a system's current structural health and provide a quantitative approach to establishing a vehicle's serviceable lifetime. This eliminates the unnecessary decommissioning of a structurally healthy aircraft based on a prior "safe-life" approach toward aircraft maintenance schedules [3]. Aerospace system retirement is not something that can be entirely avoided. However, engineers are capable of optimizing the use of costly existing systems and components, thus extending the aircraft's lifetime beyond the original design prediction.

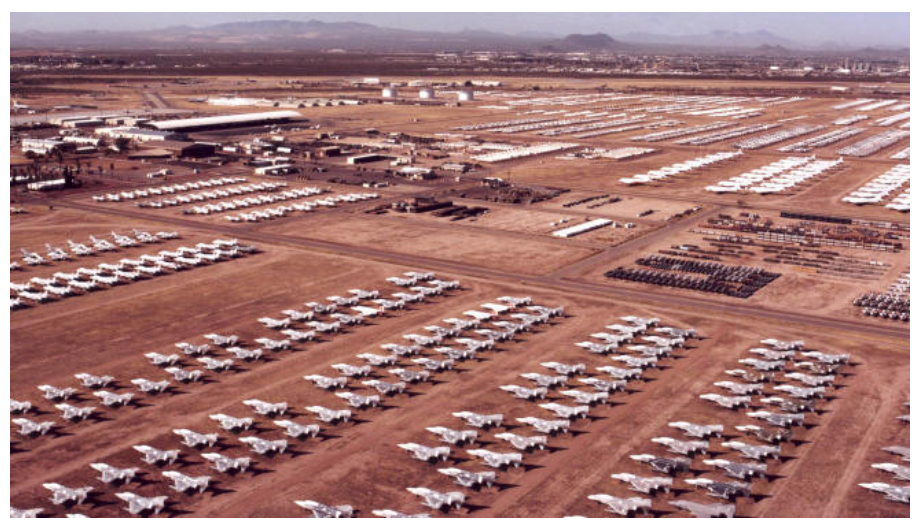

Figure 1-1-2: Military aircraft graveyard 
Supplemental benefits of a SHM system include the cost savings due to maintenance which becomes significant over the serviceable lifetime of an aerospace structure. In a study conducted by the U.S. Air Force, an average of $\$ 1.4$ million is spent on a single comprehensive F-15E maintenance event [4]. The FAA enforces four specific inspection checks on commercial transport aircraft before the aircraft is considered flightworthy [5]. The cost for these maintenance practices totals up to $11 \%$ of the total life cycle cost of the aircraft [6]. For a fleet of 108 Boeing 737 airplanes, this amount exceeds $\$ 4$ billion over a 25 year life cycle [7].

Several motivational factors have contributed to the recent spotlight and funding for SHM and CBM systems. The principal aim of developmental research and operational success continues to point toward safer and more reliable aircraft, rotorcraft and space vehicles to better ensure the safety of the crew. Prolonging structural serviceability and improving cost effectiveness in terms of routine maintenance, repair and system reusability adds to the overall benefit of implementing more efficient and comprehensive structural health monitoring systems.

\subsection{Overview of the Structural Health Monitoring Process}

A complete chart of the structural health monitoring process is summarized in Figure 1-2-1 [8]. This chart details a comprehensive approach toward the development and implementation of a structural health monitoring system for a specific application. The process begins with an operational evaluation, during which an experimental investigation and its corresponding limitations are defined. This research focuses on the application of this health monitoring process as it pertains to vibration-based, nondestructive techniques for damage detection in a multi-part, composite structure. 


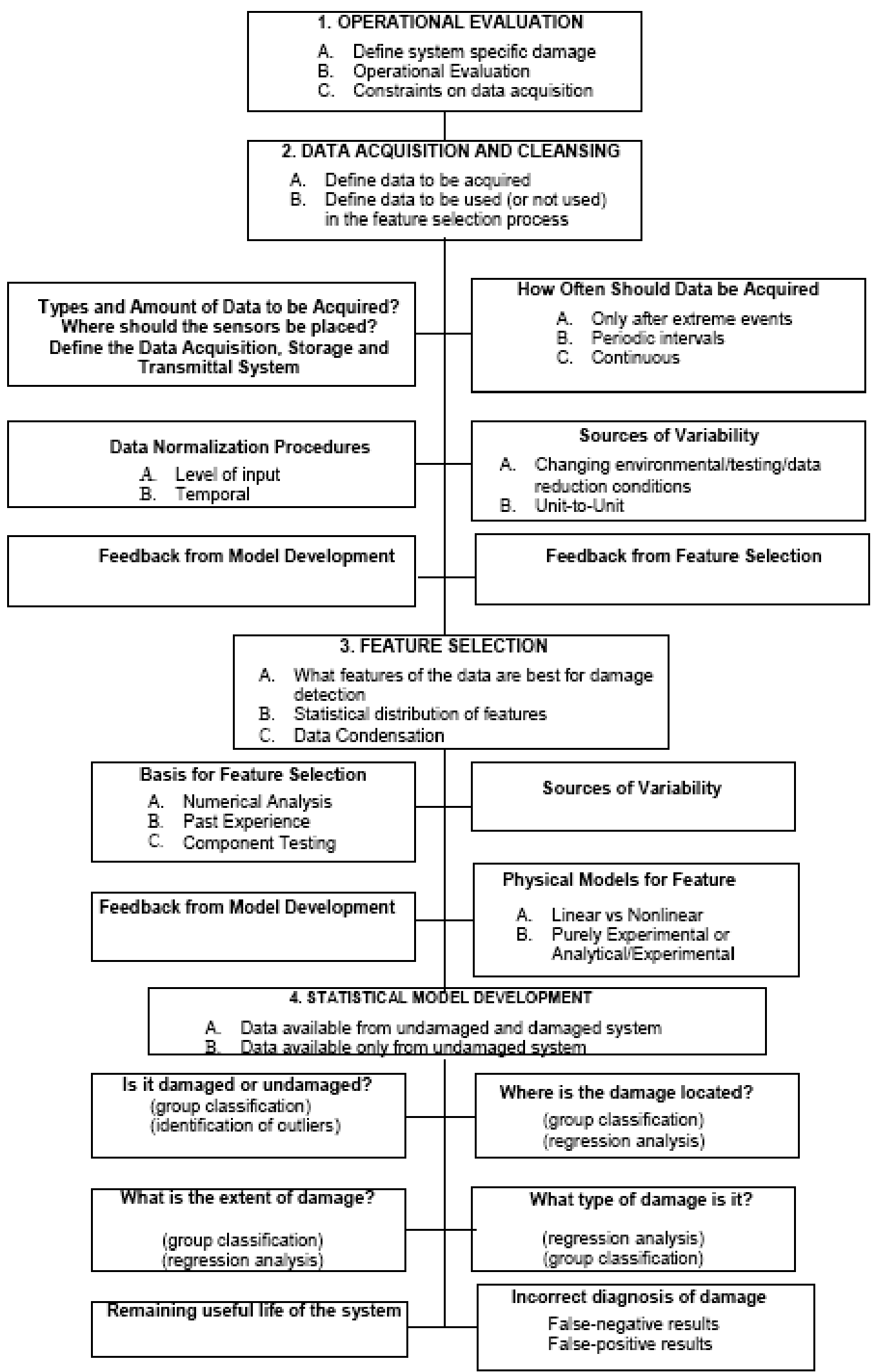

Figure 1-2-1. Flowchart, implementation of a structural health monitoring program [8] 
In a structural health monitoring application, data acquisition often ranges over a variety of damage scenarios, levels, types and quantities. Data compression is a necessity as the quantity of experimental data becomes large; a robust data compression technique can provide the ability to label key features which will give insight into damage discrimination from experimental data. Figure 1-2-1 emphasizes the importance of key features which afford insight to the identification between an undamaged and damaged system. This suggests the health monitoring system is a successful method for damage identification within the specific application.

It is often not enough to simply detect the presence of damage within a system; in multi-part systems, damage location may vary widely. Damage intensity may also vary within a system from negligible to potentially catastrophic. In addition to detection, damage localization and quantification can be achieved through the exploitation of key features to develop a comprehensive, statistical model. Statistical models provide the ability for quantitative damage interrogation within a specific structural system. An additional classification system for SHM methods is outlined by Rytter [9], which divides damage identification into four levels:

Level 1: Determination that damage is present in the structure

Level 2: Level 1 plus geometric localization of the damage

Level 3: Level 2 plus quantification of the damage severity

Level 4: Level 3 plus prediction of the remaining structure service life

The procedure outlined in Figure 1-2-1 encompasses all four levels of damage identification. 


\subsection{Previous Work and Fundamental Theory}

The earliest form of structural damage detection includes a global and/or locallyconducted visual inspection. Enhanced methods include localized experimental techniques which employ acoustic, ultrasonic, magnetic field, eddy-current, thermal field or radiography methods [10]. These methods have proven effective in experimental and operational applications, but are specifically localized to a single component or segment of a much larger structure. Further, these techniques require that the damaged component or the close proximity to damage be known for the localized experiment to be conducted successfully. This also implies that the damaged component or area must be readily accessible in order to apply the method.

Experimental and finite element efforts in the area of vibration-based damage diagnostic techniques have afforded the opportunity for quantitative damage assessments. Appropriately, the SHM community is directing new attention toward the development of comprehensive damage detection techniques which serve multiple purposes. Discerning the incidence of damage, localizing the damaged component or vicinity, distinguishing and quantifying the type of damage present are key elements to a cohesive diagnostic process.

Technological advancements in piezoelectric sensing agents have greatly contributed to the recent strides in vibration-based damage detection. Over years of structural health monitoring research and development, many vibration-based methods have been developed and employed which utilize sensing improvements [10]. Levels of success range widely and are typically application-specific. An investigation of the 
benefits and downfalls of widely-used modal parameter analysis and acousto-ultrasonic (AU) methods for damage diagnostics is included within this section.

\subsubsection{Modal parameter analysis}

The key idea behind a modal analysis approach as a damage detection method is the comparison between the structure's modal characteristics in a baseline (undamaged) condition and a secondary condition under which damage has been implemented. The structure's baseline information can be gathered experimentally or constructed analytically from identified modal parameters of the actual system. The structure will experience a shift in natural frequencies and mode shapes as damage progresses within the structure. A prominent example of a damage detection system which implements modal dynamic data analysis is NASA's space shuttle modal inspection system [2] which was developed to interrogate damage within the exterior surface under the protection of a thermal protection system. A review of damage detection methods based on modal dynamics has been compiled by Doebling, et. al [10] and Sohn, et. al [11]. Farrar, et. al [12] and Doebling, et. al [13] have shown that a structure's resonant frequencies are statistically resistant to random error sources as compared to other modal parameters. Modal analysis techniques have proven effective for a wide variety of civil, mechanical and architectural engineering applications [14] [15] [16]. The aerospace engineering community has utilized the method as well, specifically in the arena of composite materials research [17] [18].

Limitations of this method should be considered on an application-specific basis. Low sensitivity of frequency shifts due to progressive structural damage necessitates either very precise frequency measurements or limits experimentation to large levels of 
damage [13]. Further, frequency shift methods for damage diagnostics must be conducted on specimens with highly accurate, predetermined types and quantities of damage. Standard modal properties are experimentally approximated from sensor timehistory measurements; these approximations represent a form of data compression as the measurements are averaged. While data compression makes the modal properties easier to visualize, information about the current state of the experimental specimen is lost during the data reduction process. Experimentally measured modal parameters are also susceptible to changes due to fluctuating environmental conditions during a test.

For the purposes of this research, excitation frequency ranges outside the limitations of current modal parameter analysis methods. These methods utilize frequency information in the Hertz $(\mathrm{Hz})$ range; this research study intends to examine the jointed composite structure using frequencies in the $\mathrm{kHz}$ range. Since modal parameter methods do not provide high sensitivity from high frequency ranges, it is necessary to explore other means for damage detection.

\subsubsection{Acousto-Ultrasonics}

In particular, the implementation of the AU method has been popular in the structural health monitoring field primarily due to the method's non-destructive scheme for gathering acoustic wave information. Fundamentally, the AU approach attempts to detect small incidences of damage over a wide area using transducers attached to the structure. Figure 1-3-1 shows a simple AU schematic within an arbitrary structure. Sanders, et. al [19] investigated a similar experimental setup using a laminated composite beam and discovered good agreement between the experimental results and predictions. Purekar [20] and Mahapatra, et. al [21] have proven experimentally and through finite 
element means that the AU method can successfully capture the dynamics of a delaminated composite structure in structural health monitoring applications.

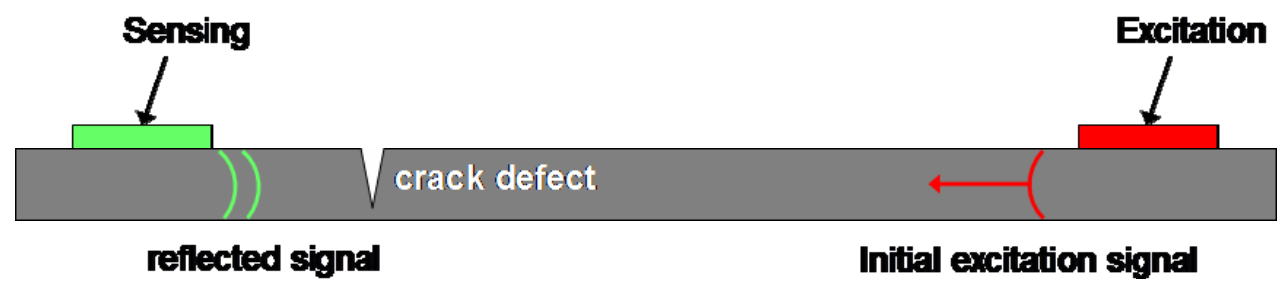

Figure 1-3-1. Acousto-Ultrasonic schematic within a structure.

Interrogation waves originate upon excitation at a singular point on the system. These waves propagate through the system and pass through any defects, whether due to structural cracks, composite delamination or thickness changes. In a bolted joint specimen, a possible damage scenario could be inherent to the joint, potentially as damage due to torque loss within one or more of the bolts; this research study suggests damage due to bolt torque loss within the composite lap joint. Active sensors located on the structure continually monitor the excitation waves during propagation and reflection about the structure.

Limitations of AU arise from the method's inherent goal of detecting small damages over large areas. There is a sensitivity exchange between detection of small damages and the ability to globally interrogate a structure. The understanding of wave propagation in a structure is paramount in the implementation of AU methods. Lamb wave propagation is typically examined for aerospace applications dealing with thin plates and is examined further in a subsequent section. 


\subsubsection{Lamb wave method}

Lamb waves classify the dispersive wave within a plate which propagates over the plate thickness and the plate width and length. Lamb wave propagation and speed are influenced by plate thickness, and thus are easily changed due to alterations in the thickness due to damage. This feature proves useful in the employment of Lamb wave analysis in a damage detection methodology. Lamb wave analysis can be used along with an active smart sensing system on a variety of experimental specimens to assess its success as a damage detection method [20]. As part of an ongoing research project at the University of Maryland, Lamb wave analysis techniques have been employed on thin plates and shells made from aluminum and carbon-fiber composite materials [22]. A variety of damage types have been instigated in each specimen: machined cracks, damage due to bolt torque loss and added mass. To date, each specimen has been a single-piece structure.

In particular, the aerospace community has devoted extensive research and monetary efforts toward the development and operation of composite materials. Considering the area of structural health monitoring using AU and Lamb wave analysis techniques, it becomes necessary to build a thorough understanding of the wave propagation properties in composite materials if a successful, vibration-based damage diagnostic method is to be implemented in composite structures. While classical laminated plate theory provides insight into wave propagation within composite materials, a popular route for accurately assessing the wave characteristics in specific composite materials moves toward a finite element modeling approach. Extensive finite element research has provided insight into the wave proliferation of undamaged 
composites. Galan and Abascal [23] developed a very useful wave dispersion prediction for an arbitrary composite laminate. Unlike isotropic materials, the orthogonality of composite plates plays an important role in the direction of wave dispersion and will vary depending on the specific composite lay-up scheme. Clark and Thwaites [24] showed through experimentation the importance of fiber orientation with wave propagation and wave speed in a composite material.

Another important phenomenon to be investigated is the wave propagation in a damaged composite, and how the damaged area influences the wave dispersion characteristics. Typical defects in a composite material can include cracks, thickness inconsistencies, and delamination sites. In addition to changes in normal wave propagation, modal parameters of the damaged composite are usually examined to detect the presence of a defect, assuming the baseline modal parameters are previously known. Tan, et. al [25] experimentally observed a decrease in amplitude as wave modes traveled through a composite delamination site. This observation indicated a detectable energy loss in the wave mode as a result of the composite damage.

\subsubsection{Signal processing using wavelet transformation}

In a wide variety of studies and applications, focus has been placed on the utilization of wavelet transformation and analysis in the field of damage diagnostics as a detection device. Yen and Lin [26] introduced wavelet packet transforms as an alternative method for extracting time-frequency information from vibration signatures recorded from the aft main power transmission of a U.S. Navy CH-46R helicopter. The wavelet packets are formed by taking linear combinations of typical wavelet functions, and proved to be more successful as compared to Fourier based feature results with the 
same application. $\mathrm{Ma}$ and $\mathrm{Fu}$ [27] used the wavelet analysis method applied to Lamb wave signals in conjunction with a smart active sensing technique on a composite plate. Their experimental method showed successful crack detection of a damaged composite plate. A similar experiment was conducted by Guo and Cawley [28] which investigated the interaction of Lamb waves with delamination sites in orthogonal laminates. Their study proved the Lamb wave analysis as a successful detection means for damage due to composite delamination. Wilcox, Lowe and Cawley [29] have published an extensive review on the transducer selection process for use in Lamb wave experimental and analysis methods; the selection is heavily reliant upon the required resolution, the particular defect and the available transduction options.

\subsubsection{Interrogation of bolted composite structures}

Adaptive materials have been employed to interrogate damage within a bolted joint as damage due to bolt torque loss. Yang and Chang [30] developed a nondestructive interrogation technique which used PZT (lead zirconate titanate) embedded sensor washers to constitute a sensor network on a carbon-carbon composite thermal protection system. The smart sensor system successfully identified loosened bolts after a series of shaker verification tests which simulated a re-entry process. Another investigation by Chang, Hung and Wang [31] examined the bearing failure of laminated composite double-lap metal/composite/metal bolted joints. This particular experiment investigated pure and bolt bearing damage. The experiment concluded that shear cracks formed due to compression failure, which could be suppressed with lateral supports and clamping pressure to increase bearing strength. 


\subsection{Discrimination Techniques}

To successfully achieve a Level 2 damage prediction within a structure, there becomes a need for a damage discrimination method. Not all research studies go beyond simple detection of damage, a Level 1 prediction. Arguably, the graduation past Level 1 prediction is paramount and provides the most useful contributions to the structural health monitoring field of study. Operational practices will benefit greatly from a geometric localization discriminator associated with a specific damage diagnostic method. Further, the quantification of damage can prove to be useful when determining the need for damage repair and assessing the operational lifetime of a structural system. Many discrimination techniques are developed to be application-specific. This is due to the importance of key features which are specific to an application and are vital to the discriminator development process. Discrimination methods are also heavily based on the goals of the technique: is the aim to distinguish a damaged scenario from an undamaged scenario, to distinguish the type of damage (crack, delamination, torque loss), or is the aim to quantify of damage level? The overall discriminatory goal could also be a combination of many singular damage detection aims. Expert discrimination systems are largely application-specific and rule-based, depending on feature selection. The trends and ideas behind an expert system can make it useful to other applications that are similar in experimental set-up or damage classification. The development of damage indices has been performed by Stubbs, et. al [32] [33] and applied to aerospace engineering structures. A number of statistical methods provide a means to validate a specific expert system. Fisher discrimination techniques [34] [35] can be used to statistically validate an expert-based system. Simpler methods such as the Euclidean 
distance classifier [36] can provide one-or-the-other discrimination capabilities, most useful in the distinction between damaged and undamaged scenarios. In this research study, a damage index is adopted and used as the key feature in damage detection and localization for a jointed composite specimen; this damage index is clearly defined in a subsequent chapter. Simple statistical means provide a method to reach Level 3 damage prediction due to bolt torque loss.

\section{5 $\quad$ Research Aim}

This research study utilizes the Lamb wave and smart active sensing method on a multi-part structure, consisting of composite plates and a bolted composite joint. It is the aim of this research study to utilize these techniques to develop a comprehensive damage diagnostic on the jointed composite structure which will undergo damage due to bolt torque loss. The goal is specific to the experimental specimen and damage type. The ideas and results are presented with five main intentions:

1) Apply a smart sensor array and utilize Lamb wave analysis techniques on the composite jointed specimen as a means for structural health monitoring.

2) Compress experimentally obtained signal information into a singular damage index for a variety of damage scenarios due to bolt torque loss in the composite jointed specimen.

3) Attain Level 1 prediction: utilize smart sensor signal response and damage index to determine the presence of damage due to bolt torque loss.

4) Attain Level 2 prediction: exploit pattern recognition of the damage index to develop a qualitative damage localization method. 
5) Attain Level 3 prediction: quantify damage due to bolt torque loss through rule-based and statistical discrimination methodology.

In combination, fulfilling these aims will successfully develop a comprehensive health monitoring system for the jointed composite structure up to a Level 3 prediction. Level 4 damage identification is typically associated with fracture mechanics, fatigue-life analysis or structural design assessment [9]. It is not the goal of this research study to achieve Level 4 damage prediction capability for the jointed composite structure. 


\section{Chapter 2: Experimental Procedure}

\section{$2.1 \quad$ Jointed Composite Panel}

The experimental specimen used in this research was prepared in the Composites Research (CORE) Laboratory of the University of Maryland at College Park. The specimen was composed of four flat Hexcel IM7/8552 carbon/epoxy composite pieces in an 8-layer [90/0/0/90]s lay-up. The experimental configuration of the jointed composite panel is shown in Figure 2-1-1. The joint was composed of 12 bolts and nuts. The distance between each bolt was 2" and each bolt was tightened using a hand-torque wrench to a value of $5 \mathrm{~N}-\mathrm{m}$. A bolt numbering scheme starting with Bolt 0 at the upper, right corner and progressed down the right side of the joint. Upon completed assembly, the jointed panel was 36 " long and 12 " wide. The panel was mounted on the left and right side to simulate a clamped-free-clamped-free boundary condition. The bottom side of the panel (unseen in Figure 2-1-1a) sat three inches from the laboratory table without obstruction. The assembly was allowed to rest unmoved in a temperature controlled laboratory at the University of Maryland. 


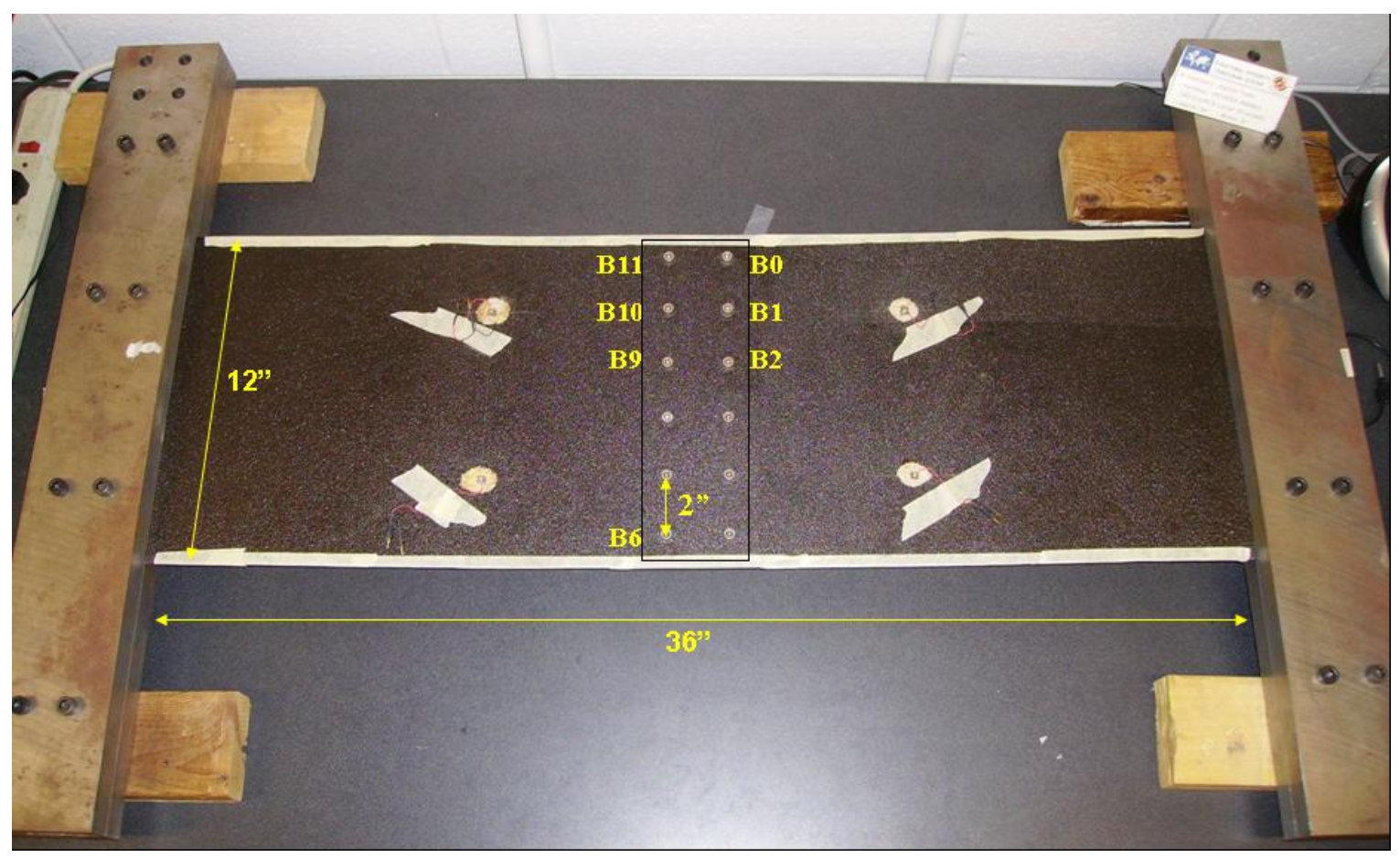

(a) Photograph of top view.

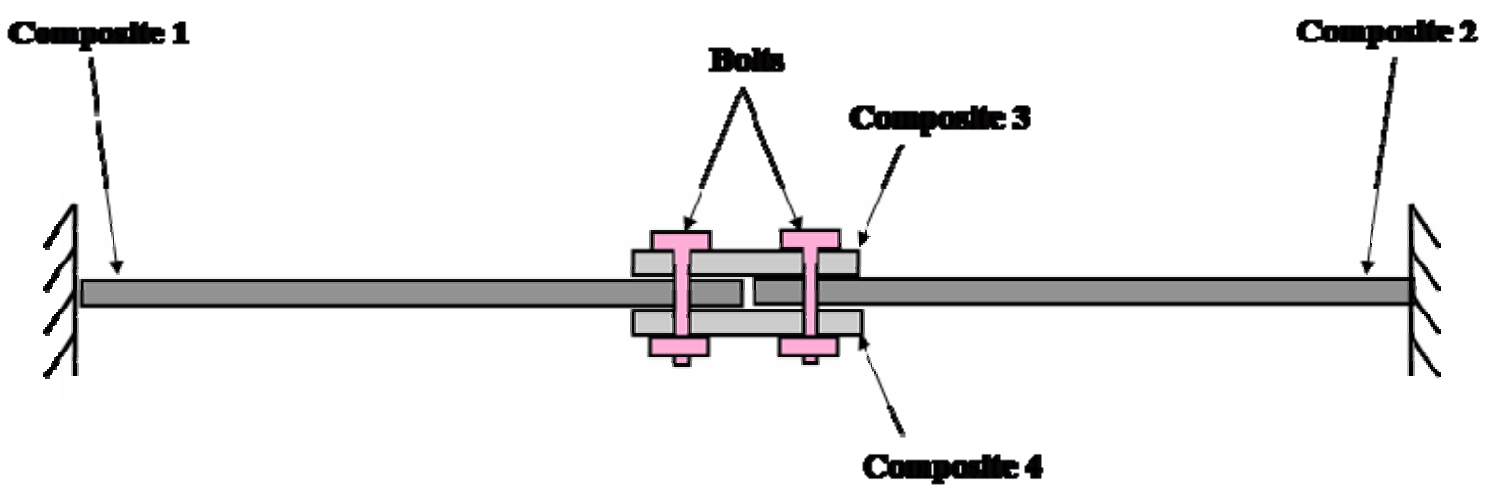

(b) side view

Figure 2-1-1: The jointed composite panel. 


\subsection{Wireless Smart Sensor System}

A square type of PZT-4 piezoelectric patch was bonded to composite panels 1 and 2; these piezo-patches are shown bonded to the jointed specimen in Figure 2-1-1 and below in Figure 2-2-1. The piezoelectric transducers were $0.25 "$ x 0.25 ". On composite 1, the center of piezo-patches 1 and 2 were placed 6" from the bolts on the left-hand side of the joint and 3" from the top and bottom edges of the experimental assembly. The patches were oriented 6" from one another. Patches 3 and 4 were situated on composite 2 in a mirrored configuration.

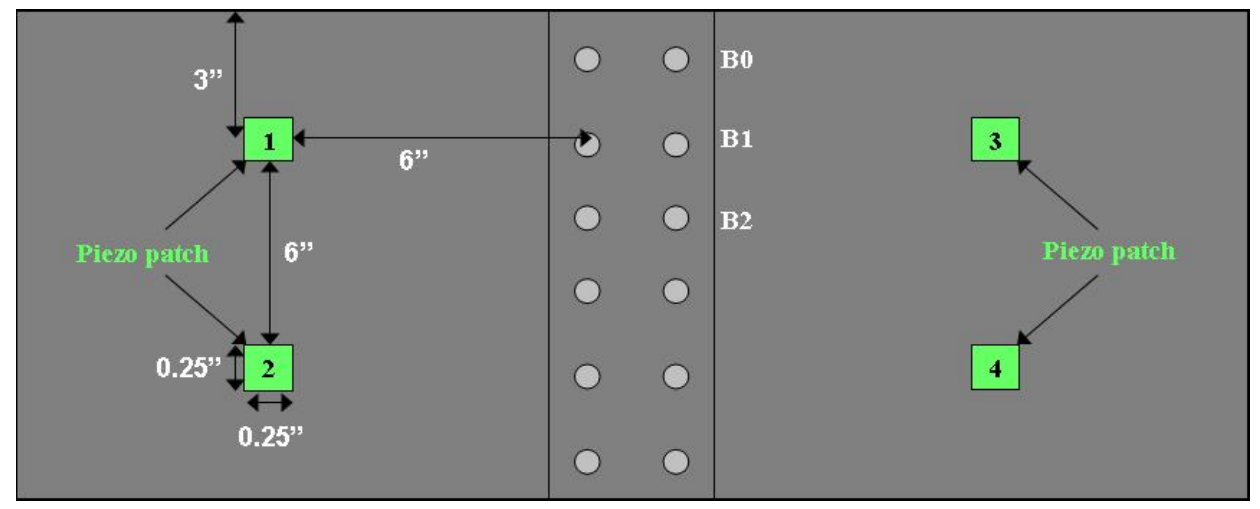

Figure 2-2-1: The jointed composite panel with piezoelectric patch assembly.

For excitation of the jointed panel, a windowed five-cycle tone burst was used at six interrogation frequencies: $20 \mathrm{kHz}, 40 \mathrm{kHz}, 60 \mathrm{kHz}, 80 \mathrm{kHz}, 100 \mathrm{kHz}$ and $120 \mathrm{kHz}$. An A/D and D/A data acquisition board (National Instruments: USB-6259) was used with a set sampling frequency of $1500 \mathrm{kHz}$. LabVIEW SignalExpress ${ }^{\mathrm{TM}}$ software created a formula excitation signal in the form of a Gaussian cosine burst signal according to the smart active sensing technique using a wavelet analysis method [30]. An example of this signal at an excitation frequency of $20 \mathrm{kHz}$ is shown in Figure 2-2-2. 


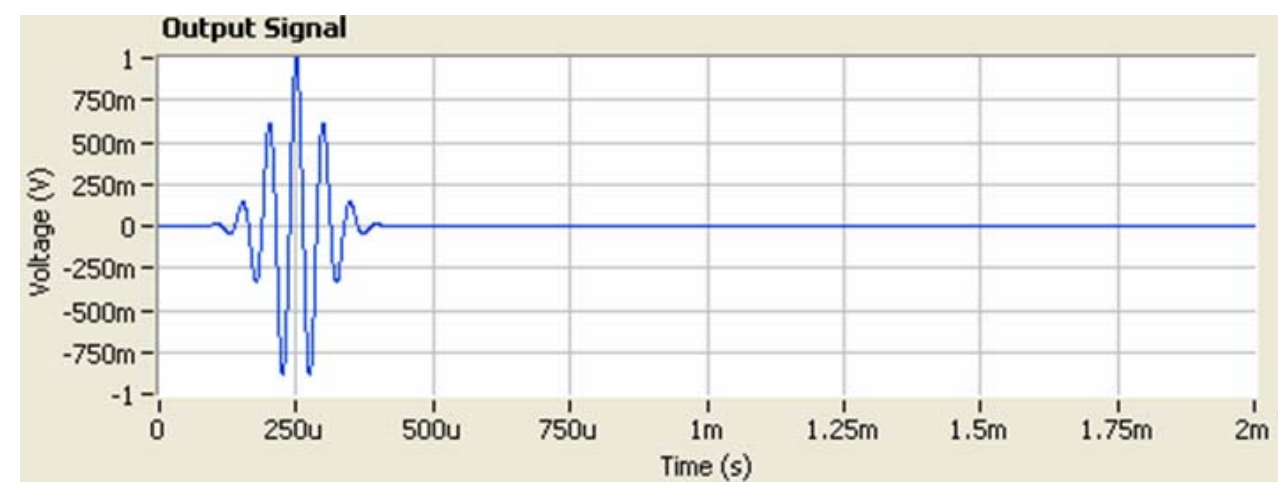

Figure 2-2-2: Formula excitation output signal with $20 \mathrm{kHz}$ excitation frequency.

A block size of 3000 samples was recorded at a sample rate of $1.500 \mathrm{kHz}$. In conjunction with the National Instruments data acquisition board, a Belkin ${ }^{\circledR}$ Bluetooth ${ }^{\circledR}$ Technology USB adapter was used to transmit signal information wirelessly. Both of these devices are shown in Figure 2-2-3.

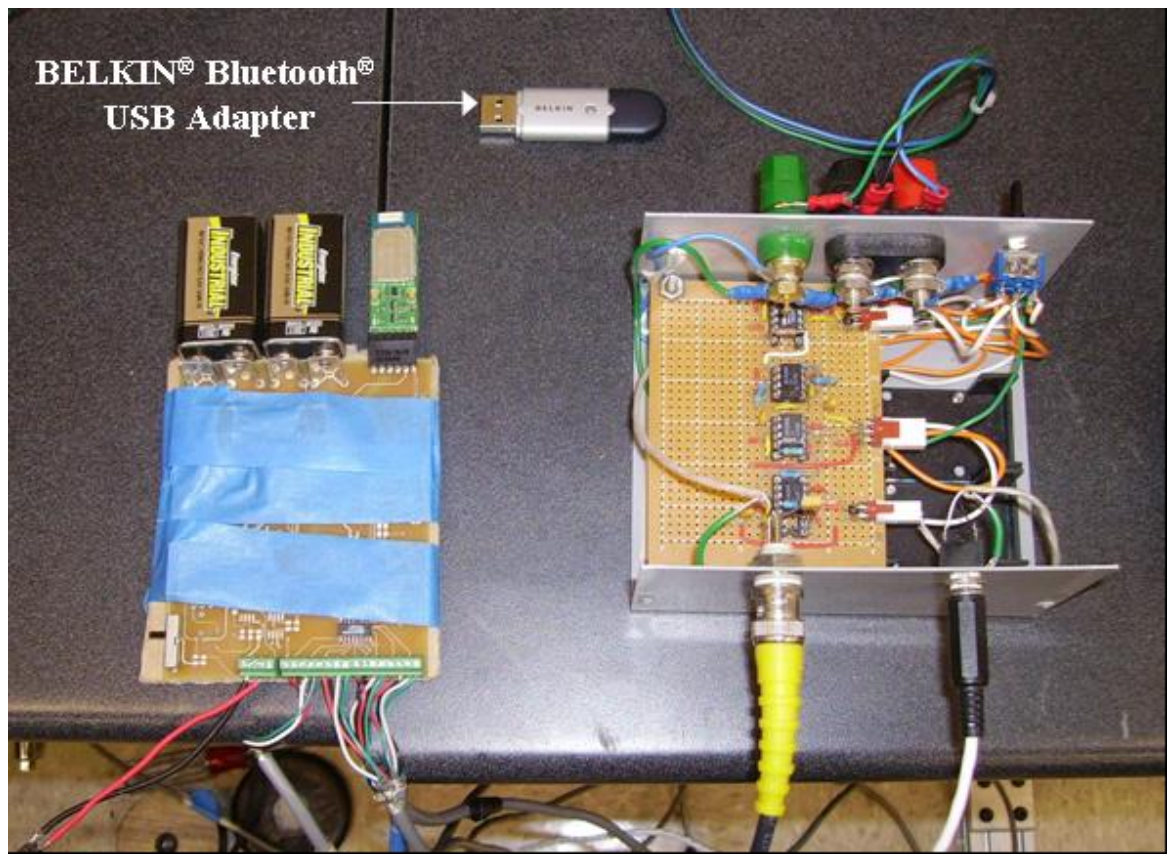

(a) National Instruments data acquisition board and Bluetooth ${ }^{\circledR}$ USB Adapter. 


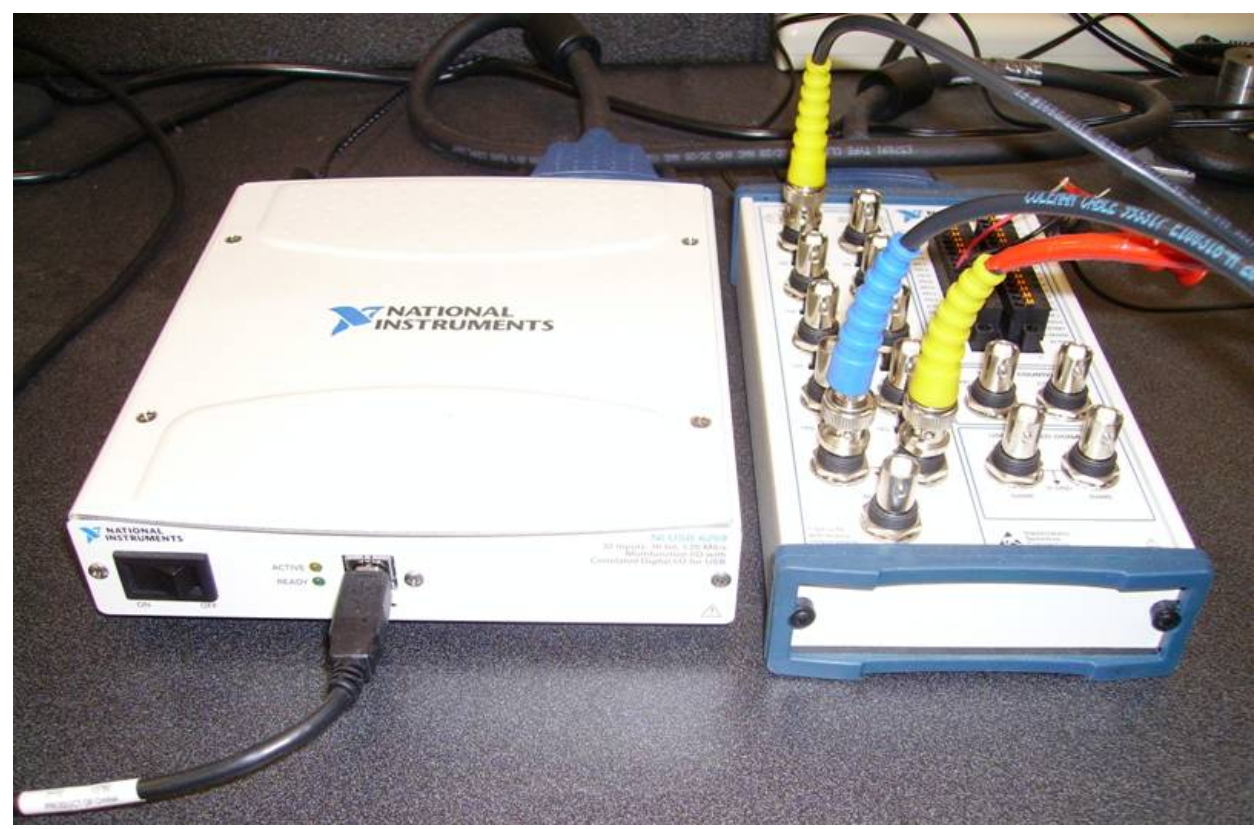

(b) National Instruments ${ }^{\mathrm{TM}}$ USB-6259.

Figure 2-2-3: Wireless data acquisition system.

For the purposes of experimentation, four excitation configuration cases were used corresponding to the numbered piezoelectric exciters (E1, E2, E3 and E4). The sensor array permitted active sensing capabilities at multiple points along the jointed composite specimen. Using one piezo-patch to excite the composite panel, time- and frequency-response information was gathered by the remaining three piezos and actively transmitted to the LabVIEW SignalExpress ${ }^{\mathrm{TM}}$ software. The jointed composite panel was separated into four quadrants, corresponding to the four piezo locations and numbers; these quadrants and the four excitation/active-sensing configuration cases are shown in Figure 2-2-4. Under this condition, the bolt-to-sensor ratio was 3:1; within each quadrant, a single piezo-patch is responsible for gathering information that would help to indicate damage within one or more of the quadrant's three bolts. 


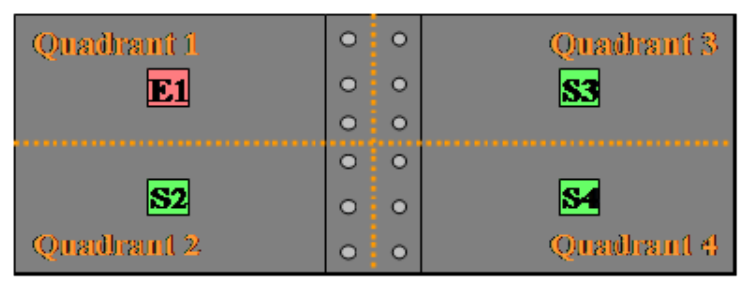

(a) Cane I

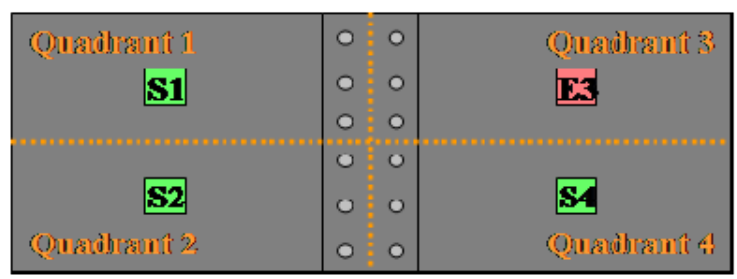

(c) Crse III

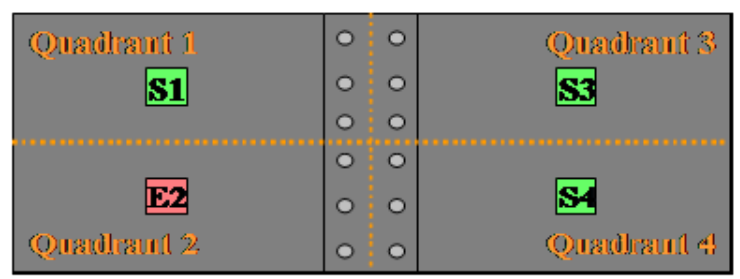

(b) Cuse II

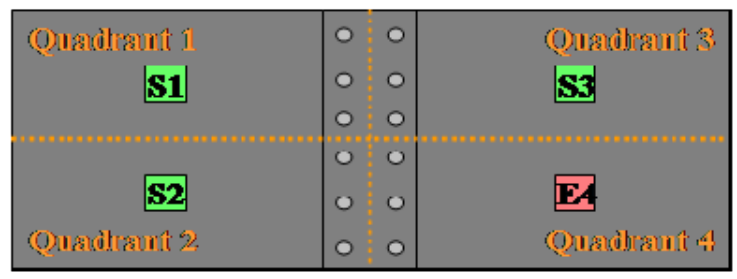

(d) Cose IV

Figure 2-2-4: Specimen quadrants and configuration Cases I, II, III, IV.

\subsection{Signal Noise Filtration and Running Average Method}

To evaluate the obstructive effects of noise on experimental method performance, $100 \mathrm{mVrms}$ and $200 \mathrm{mVrms}$ white Gaussian noise was added to the excitation tone burst input applied to the actuator piezo-patch. To smooth out the corrupted time response signal, a running-average method was imposed during collection of time-response data by the active sensors. The average number was 100 with an elapsed time of approximately two seconds. The resulting time response signal using the running average method had the effects of noise filtered out; thus, the resulting signal could give better insight into the damage severity present in the jointed composite specimen. 


\subsection{Damage Due to Bolt Torque Loss}

This research investigates the capability of the smart sensor system to detect damage due to bolt torque loss in the jointed composite panel. This represents a linear damage situation within the joint, since a loosened bolt does not change the linear-elastic properties of the composite structure. Initially, each of the 12 bolts was set to a torque value of $5 \mathrm{~N}-\mathrm{m}$ using a hand torque wrench; this initial condition represented an "undamaged" scenario within the composite specimen. To simulate a damage situation, a single bolt was intentionally loosened to percentage values of the undamaged torque: $25 \%$ torque loss $(3.75 \mathrm{~N}-\mathrm{m}), 50 \%$ torque loss $(2.5 \mathrm{~N}-\mathrm{m}), 75 \%$ torque loss $(1.25 \mathrm{~N}-\mathrm{m})$ and $100 \%$ torque loss $(0 \mathrm{~N}-\mathrm{m})$. The undamaged case and four damaged cases are implemented individually on Bolt 0 , Bolt 1 , Bolt 2 and Bolt 6 in the initial phase of experimentation. Smaller torque values were examined for Bolt 0 to test the sensitivity capabilities of the Lamb wave method: $5 \%$ torque loss $(4.75 \mathrm{~N}-\mathrm{m}), 10 \%$ torque loss $(4.5$ $\mathrm{N}-\mathrm{m}), 15 \%$ torque loss $(4.25 \mathrm{~N}-\mathrm{m})$ and $20 \%$ torque loss $(4 \mathrm{~N}-\mathrm{m})$ were examined and included in the Bolt 0 results.

A secondary experimentation phase investigated the effects of a dual-bolt torque loss configuration. Bolt pairs would undergo identical torque loss scenarios. The first bolt pair consisted of Bolt 0 and Bolt 1, each located within Quadrant 3. A second bolt pair consisted of Bolts 0 and 6, located on opposite sides of the composite joint and in diagonally oriented quadrants (Quadrant 3 and Quadrant 2). The bolt pairs were intentionally loosened to percentage values of the undamaged torque: $25 \%$ torque loss (3.75 N-m), $50 \%$ torque loss $(2.5 \mathrm{~N}-\mathrm{m}), 75 \%$ torque loss $(1.25 \mathrm{~N}-\mathrm{m})$ and $100 \%$ torque loss $(0 \mathrm{~N}-\mathrm{m})$, similar to the single-bolt experimentation. Collecting a variety of torque 
loss information using four excitation/sensing configurations provided a database of information specific to the jointed composite panel that was used to gain insight into the smart sensor array system's capability as an insightful damage detection and damage localization method.

\subsection{Data Compression: Damage Index}

For each undamaged and damaged scenario, excitation and active sensing information was gathered for Cases I, II, III and IV (excitation at E1, E2, E3 and E4) at the six aforementioned interrogation frequencies $(20 \mathrm{kHz}, 40 \mathrm{kHz}, 60 \mathrm{kHz}, 80 \mathrm{kHz}, 100$ $\mathrm{kHz}, 120 \mathrm{kHz}$ ). Six sets of three time- and frequency magnitude responses from the active sensing piezo-patches for a single configuration case yielded a total of 18 time and frequency-response data sets. Four configuration cases would bring the total number of time- and frequency magnitude response data sets to 72 . The variation of five bolt torque values at each of the four configuration cases yielded 360 time and frequency-response data sets for a single bolt consideration. The initial phase of experimentation focuses on four individual bolts (B0, B1, B2 and B6) which were each varied at $0,25 \%, 50 \%, 75 \%$ and $100 \%$ torque losses, totaling 2880 sets of time and frequency magnitude response data. A secondary experimentation phase examined smaller torque loss values in Bolt 0 (5\%, $10 \%, 15 \%$ and $20 \%$ torque loss) for the Case II configuration. This increased the total to 3072 pairs of time and frequency magnitude response data sets. Additionally, the consideration of two bolt pairs brought the total number of sensing information data sets to 4512. The large amount of data necessitated a data compression method of the timeand frequency magnitude response information gathered at each of the active sensing piezo-patches during each experimental scenario. 
According to the Lamb wave method, a total damage index (DI) was adopted [33] and used to identify, localize and quantify the damage present in the jointed composite system due to bolt torque loss. Initially, the active sensing data using the signals in time were separated into undamaged and damaged cases. At each excitation frequency, $\omega_{e}$, the time signal of the undamaged system, $x_{u}\left(t, \omega_{e}\right)$, and the time signal of the damaged system, $x_{d}\left(t, \omega_{e}\right)$ was used to calculate a damage index using the signals in time, $D I_{t}$. This damage index is defined by [22]

$$
D I_{t}=\frac{\int\left|x_{d}\left(t, \omega_{e}\right)-x_{u}\left(t, \omega_{e}\right)\right| d t}{\int\left|x_{u}\left(t, \omega_{e}\right)\right| d t}
$$

Further, the frequency magnitude of the spectrogram of undamaged systems, $\psi_{u}\left(t, \omega_{e}\right)$, and the frequency magnitude of the spectrogram of damaged systems, $\psi_{d}\left(t, \omega_{e}\right)$ was used to calculate a damage index using the frequency amplitude extracted from the spectrogram, $D I_{f}$. This damage index is defined by [22]

$$
D I_{f}=\frac{\int\left|\psi_{d}\left(t, \omega_{e}\right)-\psi_{u}\left(t, \omega_{e}\right)\right| d t}{\int\left|\psi_{u}\left(t, \omega_{e}\right)\right| d t}
$$

For improvement of the damage index sensitivity, the time and frequency magnitude response indices are combined and normalized according to [22]

$$
D I=\frac{D I_{f}}{\max \left(D I_{f}\right)} \times \frac{D I_{t}}{\max \left(D I_{t}\right)}
$$

where,

$$
\begin{aligned}
& \max \left(D I_{f}\right)=\max \left\{\max \left[D I_{f, 20 \mathrm{kHz}}, D I_{f, 40 \mathrm{kHz}}, D I_{f, 60 \mathrm{kHz}}, D I_{f, 80 \mathrm{kHz}}, D I_{f, 100 \mathrm{kHz}}, D I_{f, 120 \mathrm{kHz}}\right]\right\}_{\mathrm{S} 1, \mathrm{~S} 2, \mathrm{~S} 3, \mathrm{S4} 4} \\
& \max \left(D I_{t}\right)=\max \left\{\max \left[D I_{t, 20 \mathrm{kHz}}, D I_{t, 40 \mathrm{kHz}}, D I_{t, 60 \mathrm{kHz}}, D I_{t, 80 \mathrm{kHz}}, D I_{t, 100 \mathrm{kH} z}, D I_{t, 120 \mathrm{kHz}}\right]\right\}_{\mathrm{S} 1, \mathrm{~S} 2, \mathrm{~S} 3, \mathrm{~S} 4}
\end{aligned}
$$


As a note, for the E1 excitation case $(\mathrm{E} 1, \mathrm{~S} 2, \mathrm{~S} 3, \mathrm{~S} 4)$ the time- and frequency magnitude response at S1 does not exist since the piezo was acting as an actuator at the Quadrant 1 location. Thus, the S1 responses did not factor into (2-5-4) and (2-5-5) calculations. An average damage index at sensor $i$ location is given by [22]

$$
D I_{\text {average }, i}=\text { average }\left[D I_{20 k \mathrm{~Hz}}, D I_{40 \mathrm{kHz}}, D I_{60 \mathrm{kHz}}, D I_{80 \mathrm{kHz}}, D I_{100 \mathrm{kHz}}, D I I_{20 \mathrm{kHz}}\right]
$$

where,

$$
\begin{array}{cl}
\text { Case I: } & i=2,3,4 \\
\text { Case II: } & i=1,3,4 \\
\text { Case III: } & i=1,2,4 \\
\text { Case IV: } & i=1,2,3
\end{array}
$$

The total damage index, $D I_{\text {total }}$, proposed in this study was separated into two categories that focus on the individual sensor location. For the sensor pair located across the joint from the exciter, $D I_{\text {total, across joint, }}$ is defined as [22]

$$
D I_{\text {total, across joint }}=\text { average }\left[D I_{a, n}, D I_{a, m}\right]
$$

where,

Case I and II: $\quad m=3, n=4$

Case III and IV: $\quad m=1, n=2$ 
In each configuration case, combining all sensor data into a single total damage index, $D I_{\text {total, all sensors, }}$ is defined as [22]

$$
D I_{\text {total, all sensors }}=\text { average }\left[D I_{a, n}, D I_{a, m}, D I_{a, j}\right]
$$

where,

$$
\begin{array}{ll}
\text { Case I: } & j=2, m=3, n=4 \\
\text { Case II: } & j=1, m=3, n=4 \\
\text { Case III: } & j=4, m=1, n=2 \\
\text { Case IV: } & j=3, m=1, n=2
\end{array}
$$

This process is automated using a MATLAB code which is included in the Appendix. Utilizing the Lamb wave method and experimental damage index for the jointed composite system, this research study aims to fulfill three chief goals: damage indication, damage localization within the specimen's quadrants, and damage quantification within the identified quadrant.

\subsection{Baseline Sensing Variation Over Time}

A supplemental investigation took advantage of the large quantities of undamaged "baseline" time response data taken over the course of the research study. Over five months (October 2007 to February 2008) the undamaged data sets were compiled and compared against one another to examine the effects of time on the piezo-actuating and sensing capabilities and the overall experimental method. For experimental repeatability, 
it would be undesirable to notice that an aging piezo-patch yields significantly altered results over time when used on a specific composite specimen. If a noticeable change does occur, it is wise to predict the shift in data response and account for such a change over time in order to more accurately examine experimental results. To note any significant changes, the difference in baseline time response data was quantified and examined over the five months of experimentation.

\section{7 $\quad$ Baseline Sensing Variation due to Temperature Increase}

Another supplemental investigation was carried out to investigate the baseline variation and sensing capabilities of the piezo-patches due to temperature effects in the research lab. This issue has the potential to become more impacting in an operational environment that is subject to large temperature variation; the jointed composite specimen in this research study would only experience a variation of $10^{\circ} \mathrm{F}$ throughout the months of experimentation. To impose more elevated temperatures on the active sensor, a heat gun was used to elevate the temperature of the piezo-patch in Quadrant 1 from $22^{\circ} \mathrm{C}$ (room temperature) to $30^{\circ} \mathrm{C}, 40^{\circ} \mathrm{C}, 50^{\circ} \mathrm{C}, 60^{\circ} \mathrm{C}, 70^{\circ} \mathrm{C}$, and $80^{\circ} \mathrm{C}$. The panel was excited using the same aforementioned formula signal with excitation frequencies of 20 $\mathrm{kHz}, 40 \mathrm{kHz}, 60 \mathrm{kHz}, 80 \mathrm{kHz}, 100 \mathrm{kHz}$, and $120 \mathrm{kHz}$ at the piezo-actuator in Quadrant 2. All 12 bolts remained at their full undamaged torque value of $5 \mathrm{~N}-\mathrm{m}$ for the entire investigation. Active sensing information in the form of time- and frequency magnitude response was gathered from Quadrant 1; the path of excitation and the sensing location is shown in Figure 2-7-1. 


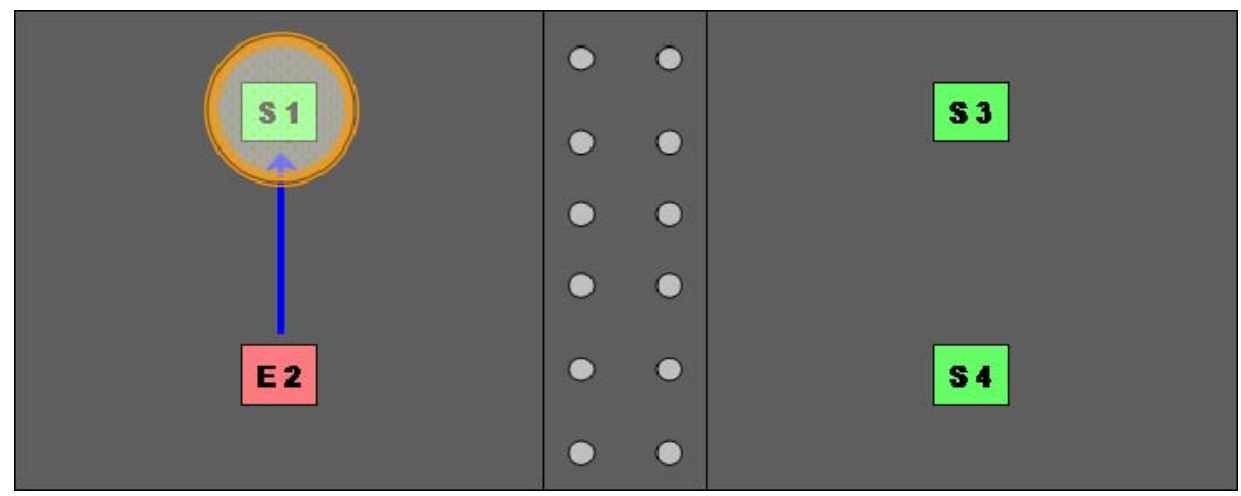

Figure 2-7-1: Baseline temperature variation of S1, E2 excitation. 


\section{Chapter 3: Damage Due to Bolt Torque Loss}

\subsection{Bolt 0 damage due to torque loss}

In this section, the torque of Bolt 0 was manipulated to evaluate the damage detection capabilities of the smart sensor array system on the jointed composite panel. Five torque values were tested at each of the four excitation configuration cases.

\subsubsection{Bolt 0, Excitation Case I}

Using the piezo-patch in Quadrant 1 (E1) for excitation of the jointed composite panel, sensing information is compiled at the three remaining sensors (S2, S3, S4) located within the remaining three quadrants. Figure 3-1-1 displays the excitation and sensing configuration and indicates the Bolt 0 location of simulated damage. Figure 3-1-2 represents the time response signals at S2, S3 and S4 sensors with respect to Bolt 0 torque loss at a $40 \mathrm{kHz}$ excitation frequency. Each figure displays the damaged scenario time response as compared to the undamaged (all bolts at $5 \mathrm{~N}-\mathrm{m}$ ) time response, for the purposes of overlap comparison over the signal duration.

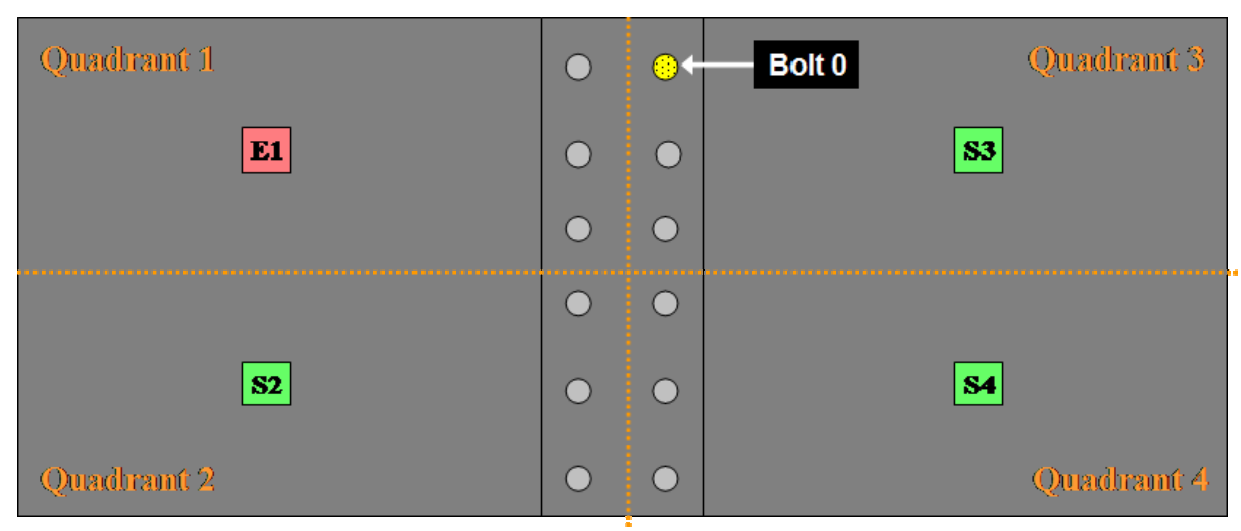

Figure 3-1-1. Excitation Case I, Bolt 0 torque loss. 


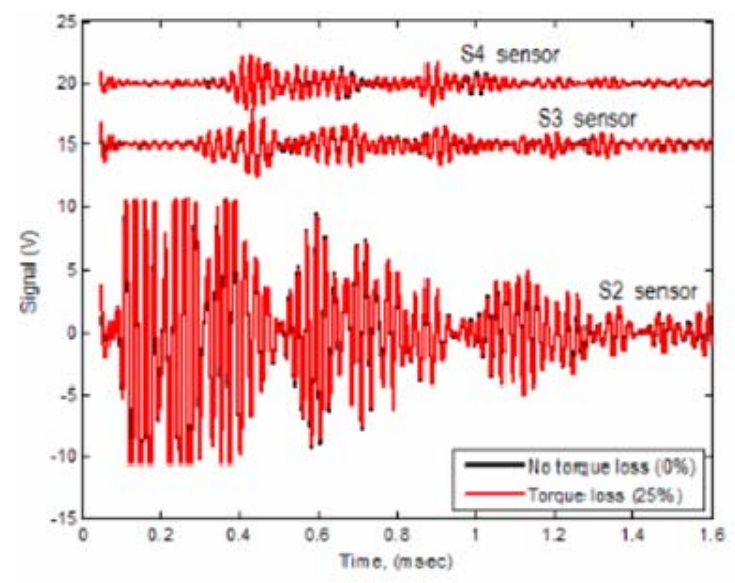

a) $25 \%$ torque loss

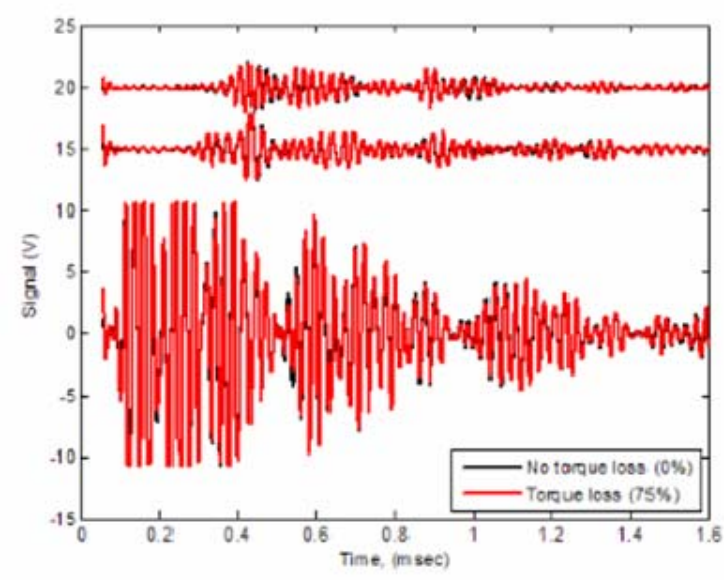

c) $75 \%$ torque loss

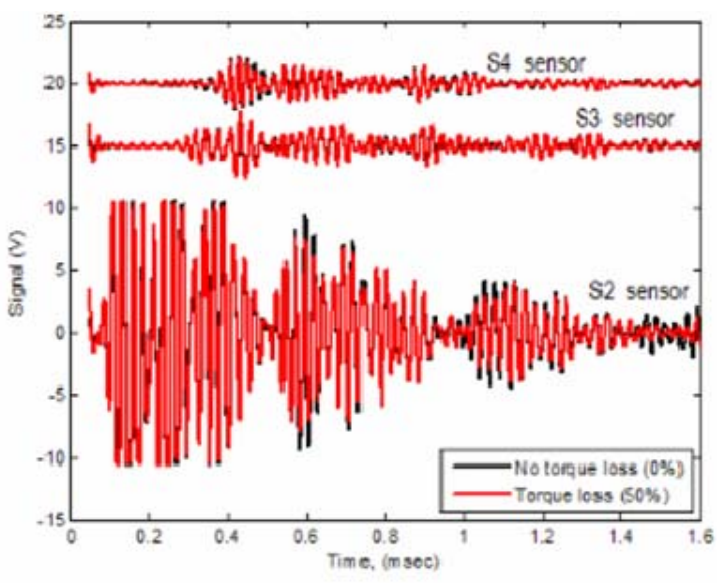

b) $50 \%$ torque loss

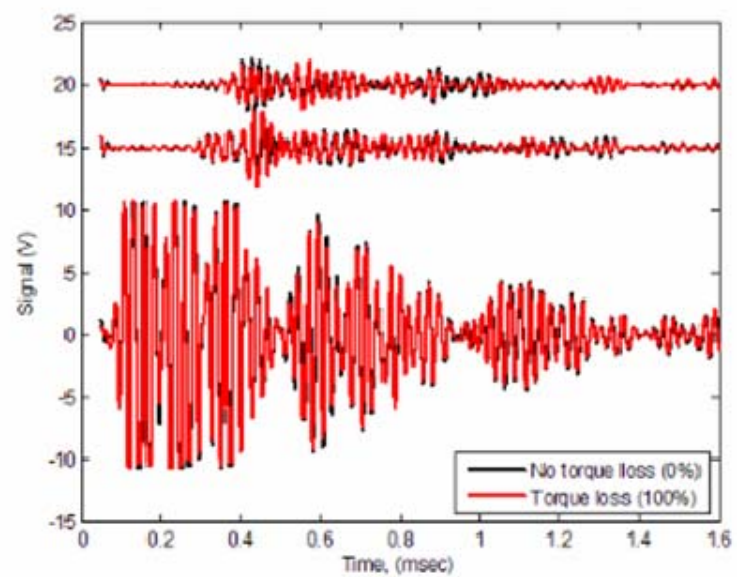

d) $100 \%$ torque loss

Figure 3-1-2. Sensing time response, B0 torque loss, E1 $40 \mathrm{kHz}$ excitation.

The magnitude of the time response signal from the S2 sensor location is noticeably greater than the sensor time response signal at locations S3 and S4. This higher magnitude occurs due to the sensor's shared panel location as the actuator. Since E1 and S2 are both located on composite 1, the majority of incident and reflected signals are measured without a great signal loss. The comparably smaller S3 and S4 time response signals are located on composite 2, across the bolted joint. Since the actuation signal must pass through the composite joint area consisting of two flat panels (composite 
3 and composite 4) and a series of tightened bolts, the incident signal magnitudes suffer a loss resulting in the smaller signal magnitude as compared to the S2 signal magnitude. While smaller in magnitude, the signals at S3 and S4 still provide insight toward the identification of damage within the jointed composite panel, as shown by their difference between undamaged and damaged time response signals.

The difference between the undamaged and damaged time response signals, shown in Figure 3-1-2 as the black and red lines, respectively, represents the scattering signal for a $40 \mathrm{kHz}$ excitation frequency under E1 actuation. This scattering signal is plotted separately and shown in Figure 3-1-3. The same scattering signal is normalized and shown in Figure 3-1-4. The signal scattering at S3 and S4 sensors increase in magnitude as torque loss increases. 


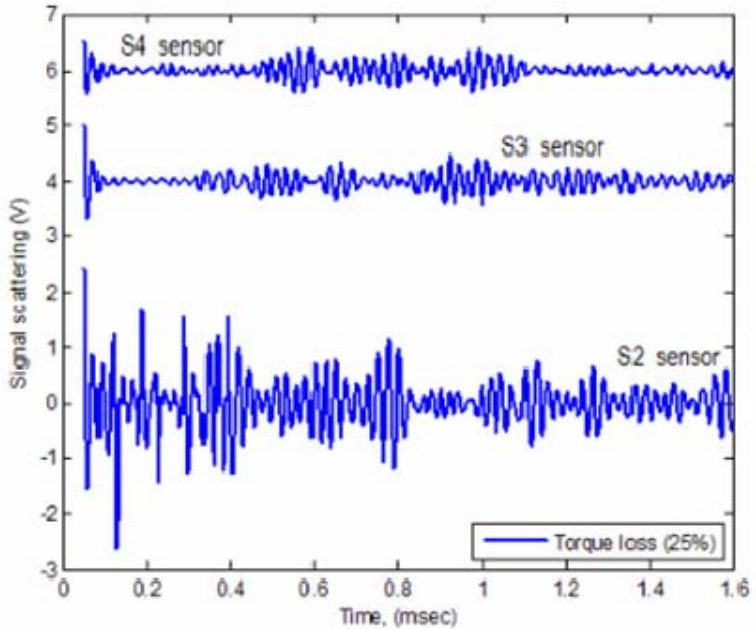

a) $25 \%$ torque loss

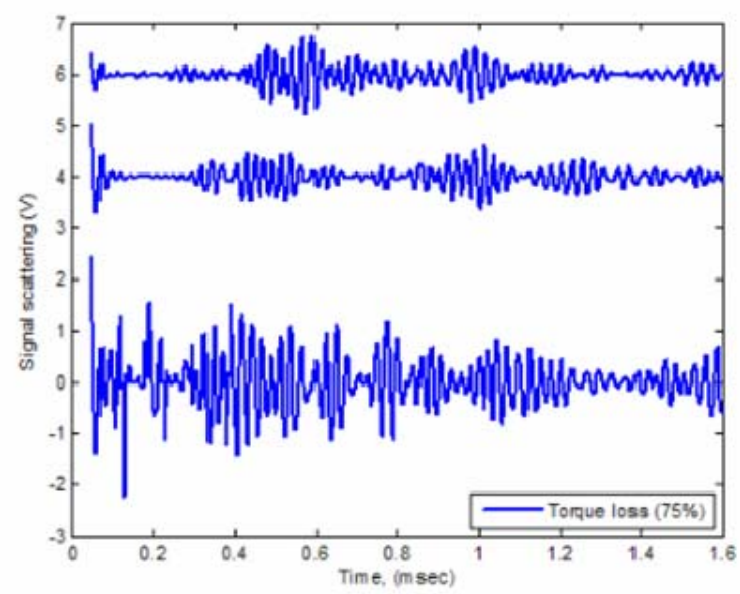

c) $75 \%$ torque loss

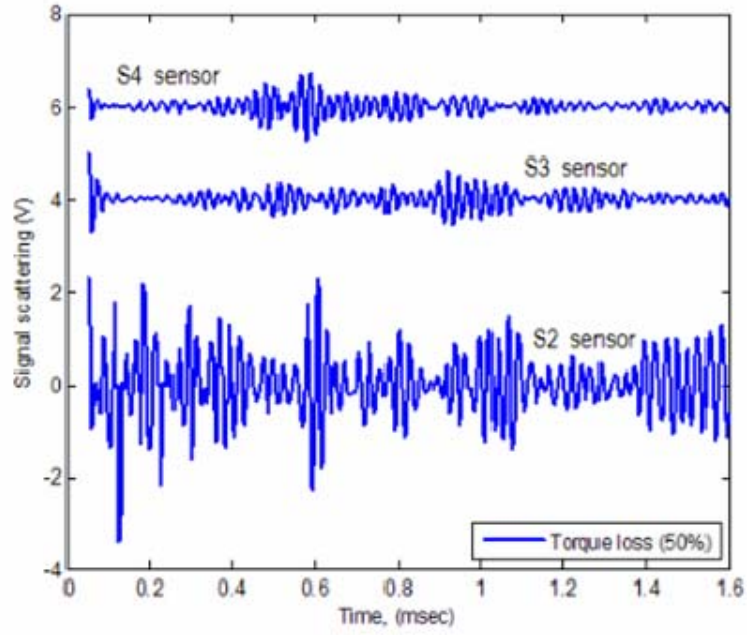

b) $50 \%$ torque loss

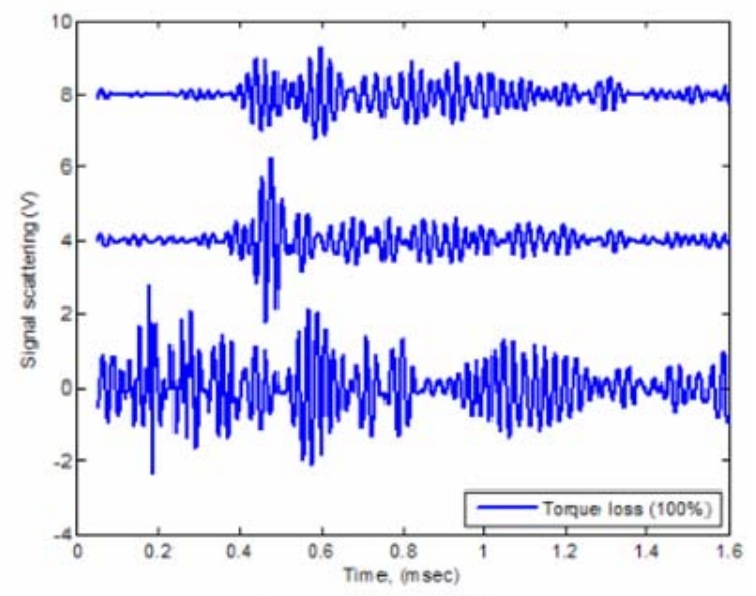

d) $100 \%$ torque loss

Figure 3-1-3. Scattering signals, B0 torque loss, E1 $40 \mathrm{kHz}$ excitation. 


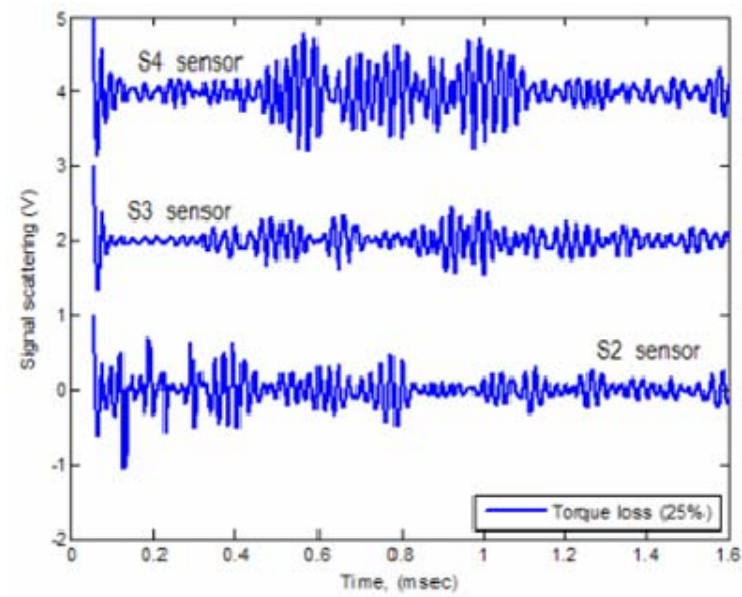

a) $25 \%$ torque loss

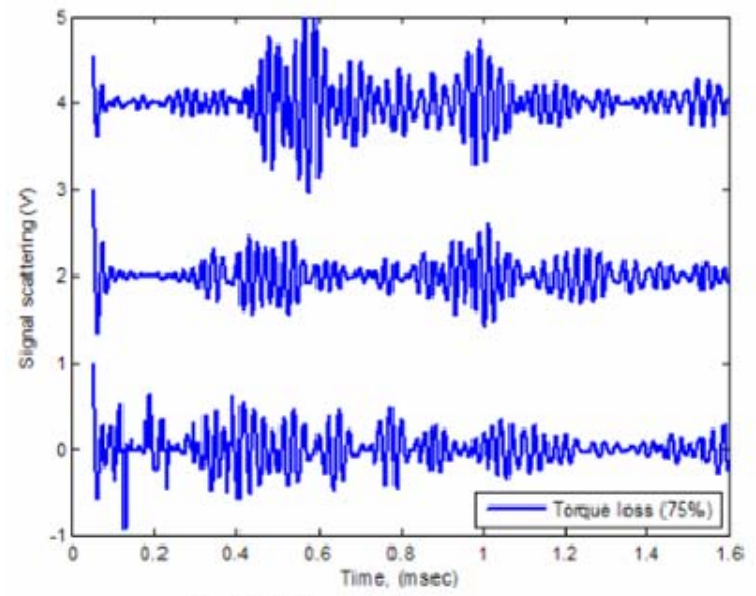

c) $75 \%$ tonque loss

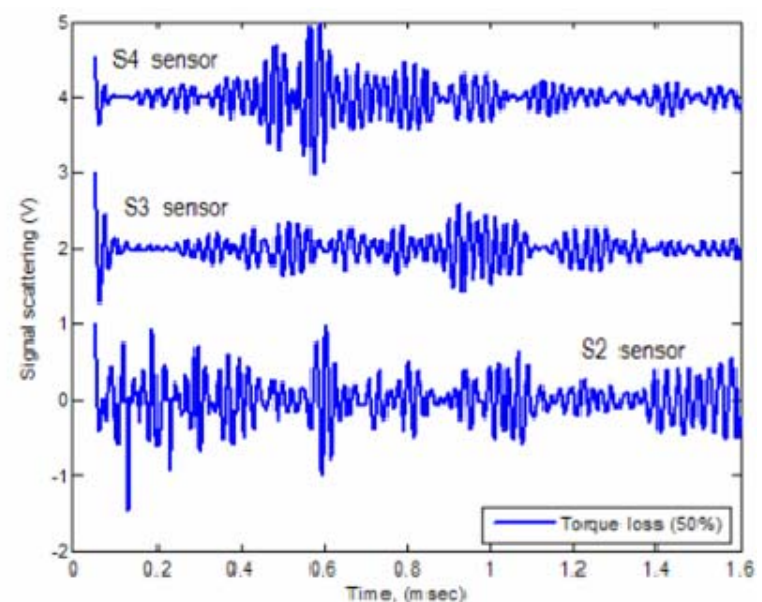

b) $50 \%$ torque loss

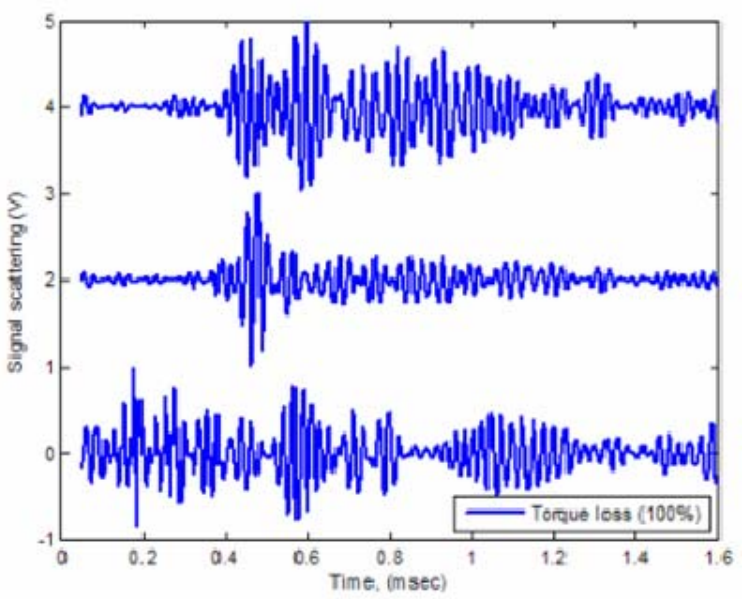

d) $100 \%$ tonque loss

Figure 3-1-4. Normalized scattering signals, B0 torque loss, E1 $40 \mathrm{kHz}$ excitation.

Figure 3-1-5 presents the damage index using the energy magnitudes due to frequency response $\left(D I_{f}\right)$ at $\mathrm{S} 2, \mathrm{~S} 3$ and $\mathrm{S} 4$ under E1 excitation. Figure 3-1-6 presents the damage index using the signals in time $\left(D I_{t}\right)$ at $\mathrm{S} 2, \mathrm{~S} 3$ and $\mathrm{S} 4$ sensing locations. For each, the damage index variation increased with bolt torque loss. There was less variation in damage index over the range of excitation frequencies. The damage index is more sensitive to changes in damage level as compared to changes in excitation frequency. 


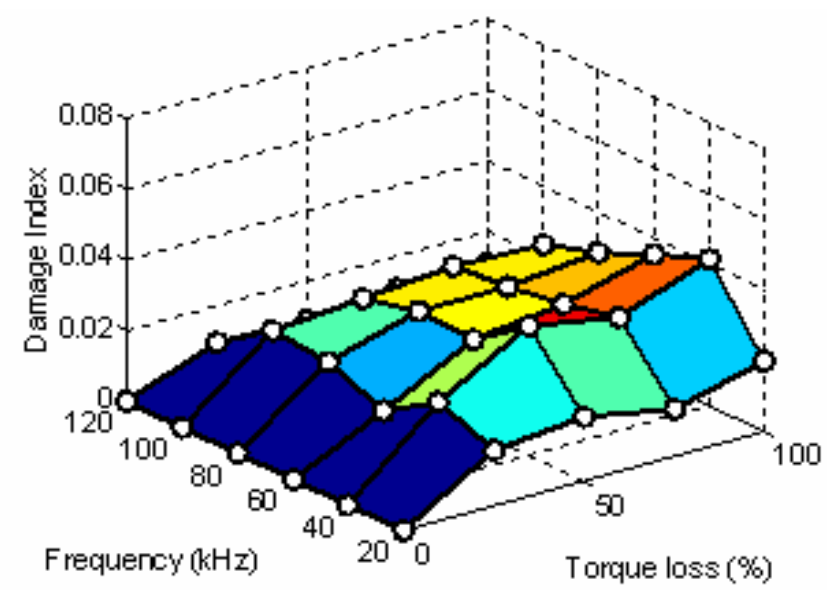

(a) S2 sensor

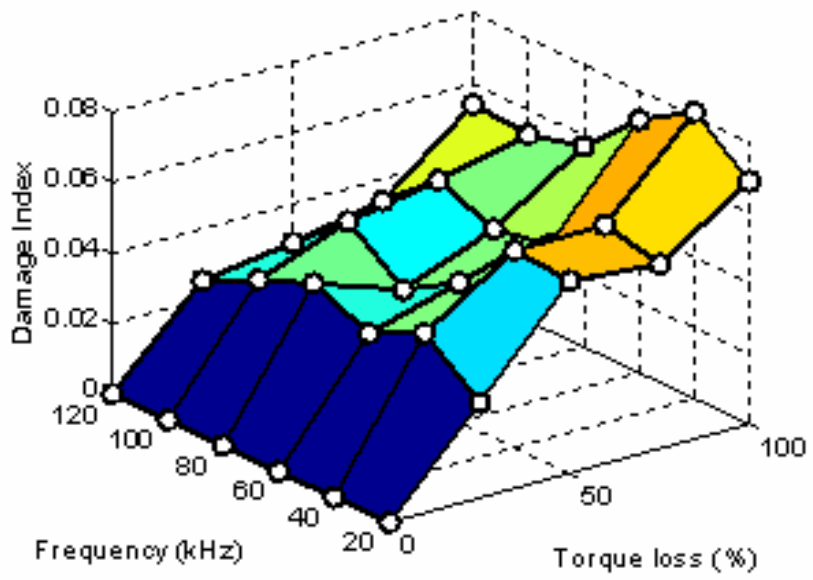

(b) S3 sensor

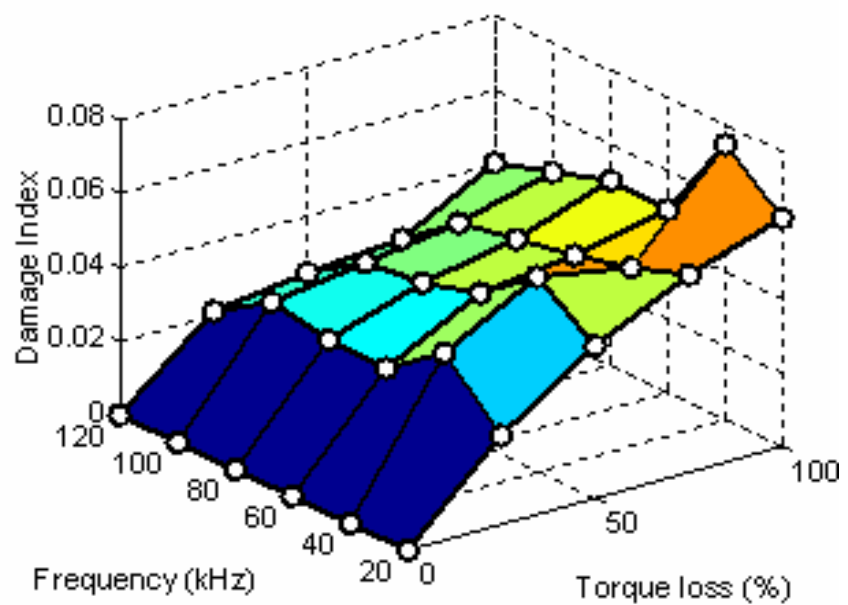

(c) S4 sensor

Figure 3-1-5. Damage index using the energy magnitudes in frequency vs. B0 torque loss and excitation frequency, E1 excitation. 


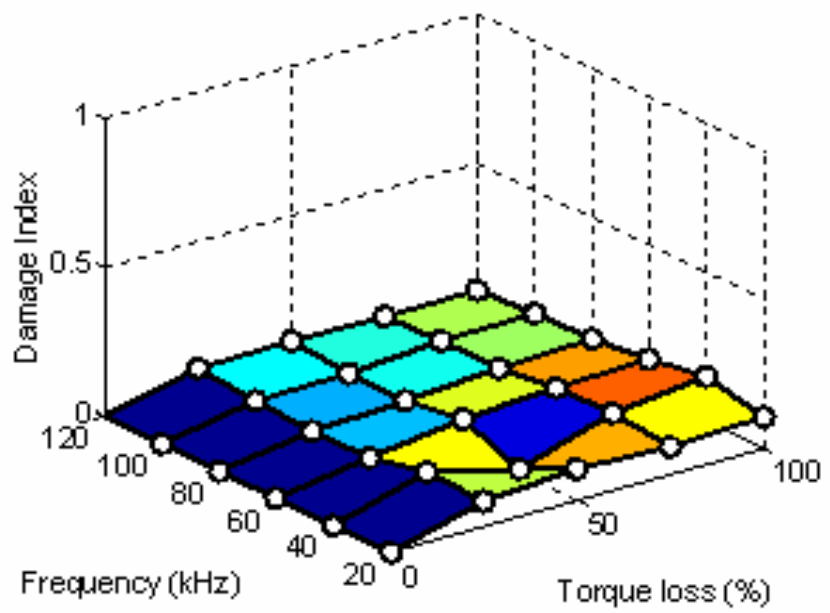

(a) S2 sensor

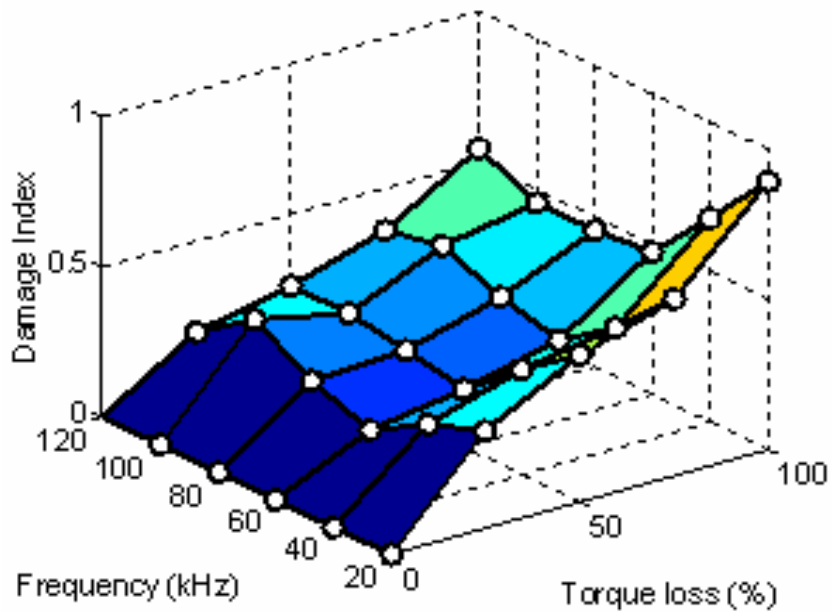

(b) S3 sensor

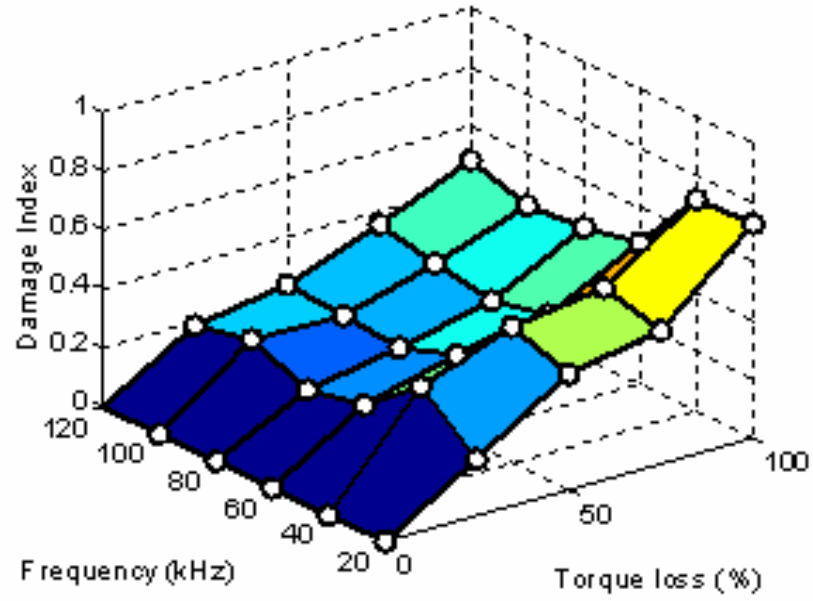

(c) S4 sensor

Figure 3-1-6. Damage index using the signals in time vs. B0 torque loss and excitation frequency, E1 excitation. 
Figure 3-1-6 displays smaller damage sensitivity in S2 as compared to the damage sensitivity in S3 and S4 sensors. This trend presents the idea that sensors located on the same panel as signal actuation will have a decreased ability to detect damage present within the bolted composite joint.

Figure 3-1-7 presents the normalized average damage index (DI average) of the jointed composite panel due to torque loss in Bolt 0 for E1 excitation. The details of this calculation are outlined in the previous chapter. The trend indicates an immediate increase in damage index from the undamaged scenario to the scenario of smallest damage, a torque loss of $25 \%(3.75 \mathrm{~N}-\mathrm{m})$. The damage index as a damage detection method is capable of indicating such small levels of torque loss within the composite joint specimen. The trend line due to S2 sensor is lower than the trend lines due to the S3 and S4 sensors. This presents a second indication of the S2 sensor location's decreased ability to perceive damage within the composite joint. The magnitude of average damage indices from sensors S3 and S4 are similar for each torque loss value; the S3 damage index is slightly larger in magnitude as compared to the S4 sensor index. 


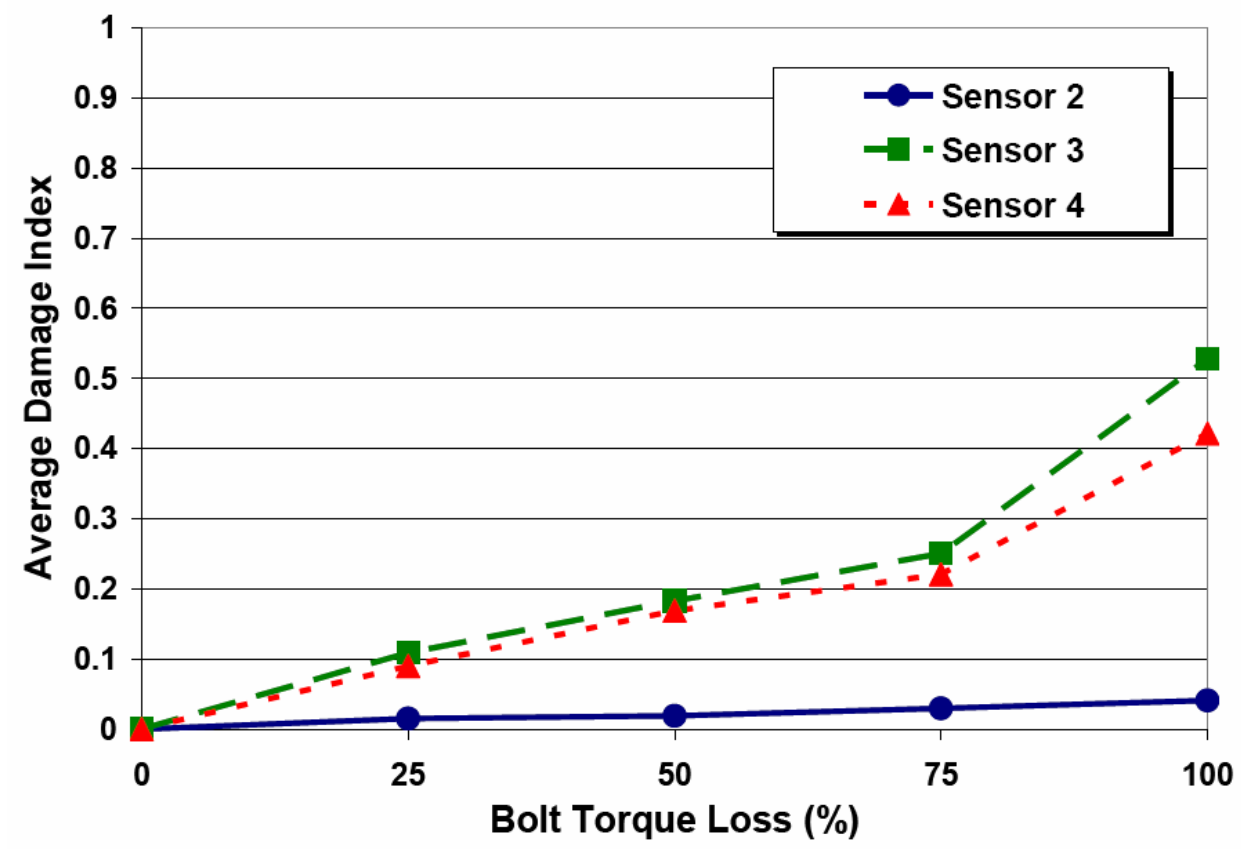

Figure 3-1-7. Average damage index, B0 torque loss, E1 excitation.

Figure 3-1-8 presents the total damage index as outlined in Equations 2-5-7 and 2-5-8. The total damage index contribution from the S3 and S4 sensors across the joint makes up nearly the entire damage index contribution from all three sensors, S2, S3 and S4. This further indicates the importance of sensing capability on panels across the composite joint when damage is due to bolt torque loss within the joint. 


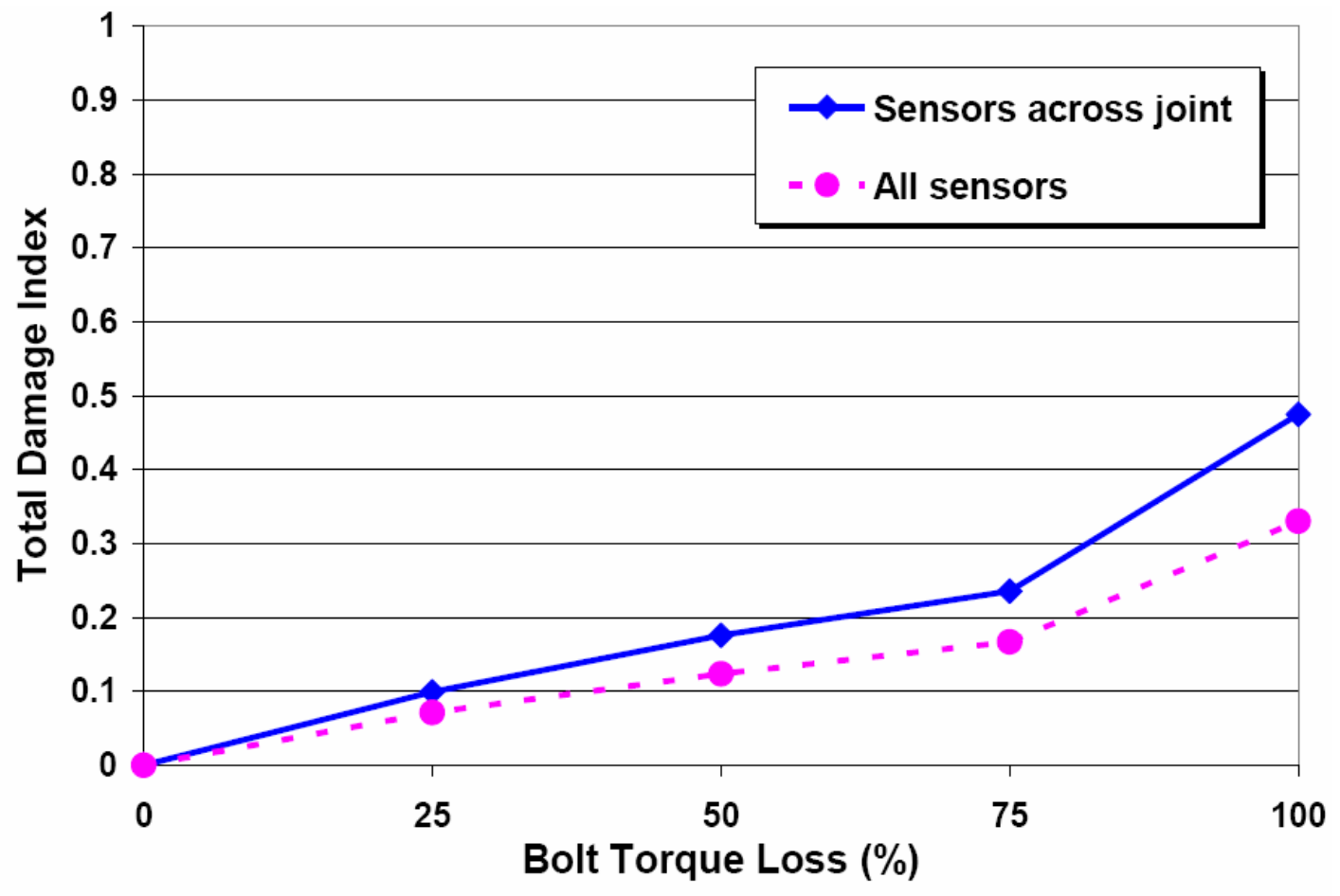

Figure 3-1-8. Total damage index, B0 torque loss, E1 excitation.

\subsubsection{Bolt 0, Excitation Case II}

Using the piezo-patch in Quadrant 2 (E2) for excitation of the jointed composite panel, sensing information is compiled at the three remaining sensors (S1, S3, S4) located within the remaining three quadrants. Figure 3-1-9 displays the excitation and sensing configuration and indicates the Bolt 0 location of simulated damage. Figure 3-1-10 represents the time response signals at $\mathrm{S} 1, \mathrm{~S} 3$ and $\mathrm{S} 4$ sensors with respect to Bolt 0 torque loss at a $40 \mathrm{kHz}$ excitation frequency. 


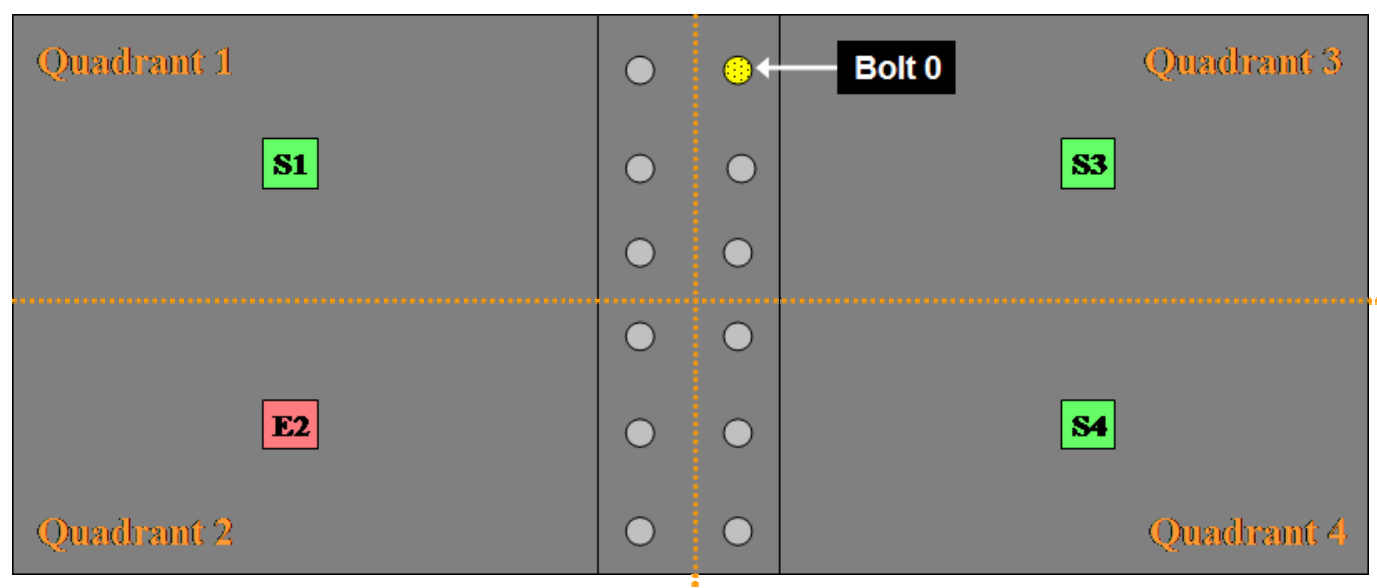

Figure 3-1-9. Excitation Case II, variable Bolt 0 torque loss.

As shown in Figure 3-1-10, the magnitude of the time response signal from the S1 sensor location is noticeably greater than the sensor time response signal at locations S3 and S4. This higher magnitude occurs due to the sensor's shared panel location as the actuator, as noted similarly in the Case I configuration with S2. The comparably smaller S3 and S4 time response signals are located on composite 2, across the bolted joint. Again, this emphasizes the incident signal magnitudes loss as a result of the signal's journey through the composite joint area consisting of two flat panels (composite 3 and composite 4) and a series of tightened bolts.

Figure 3-1-11 shows the scattering signal for a $40 \mathrm{kHz}$ excitation frequency under E2 actuation. The same scattering signal is normalized and shown in Figure 3-1-12. The signal scattering at S3 and S4 sensors increase in magnitude as torque loss increases. 


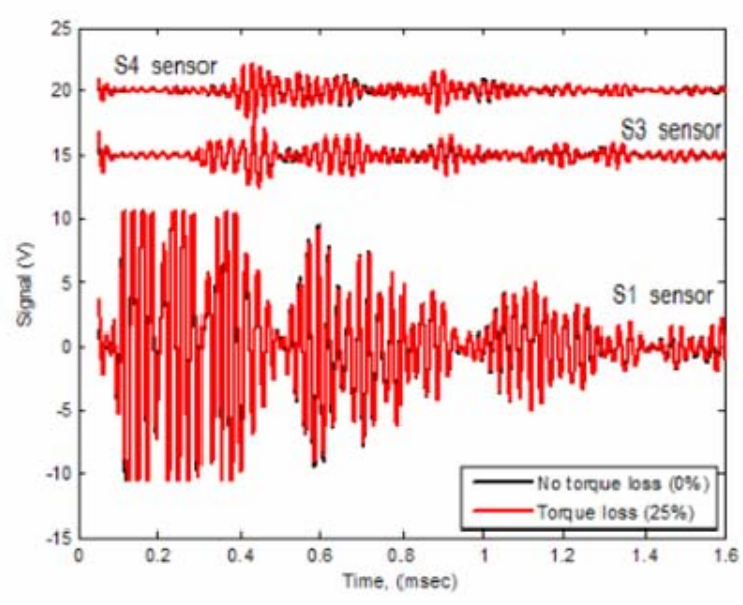

a) $25 \%$ torque loss

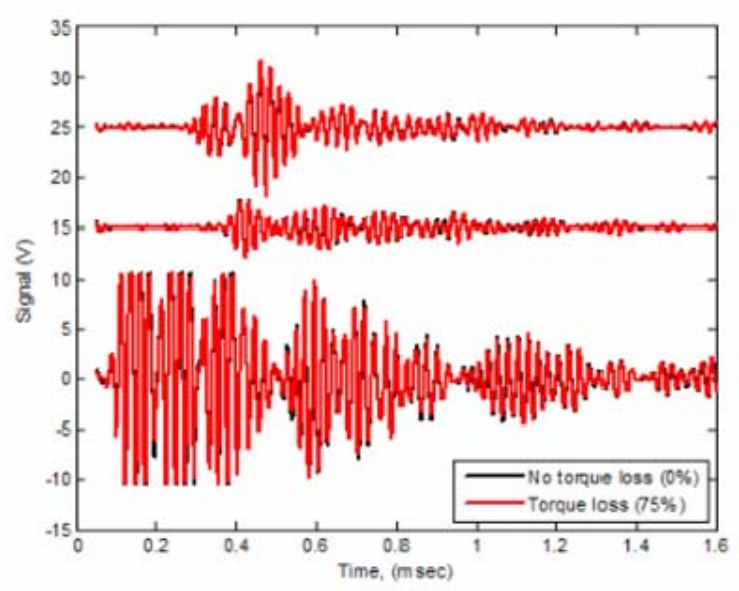

c) $75 \%$ torque loss

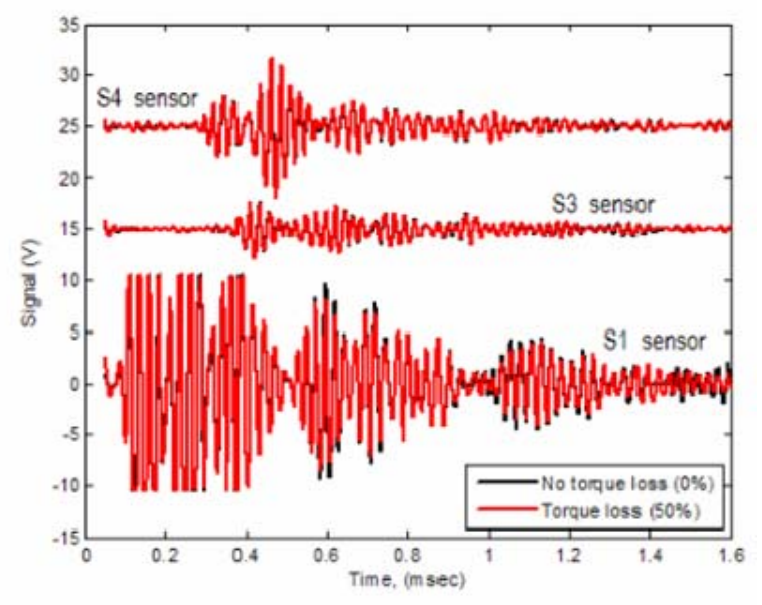

b) $50 \%$ torque loss

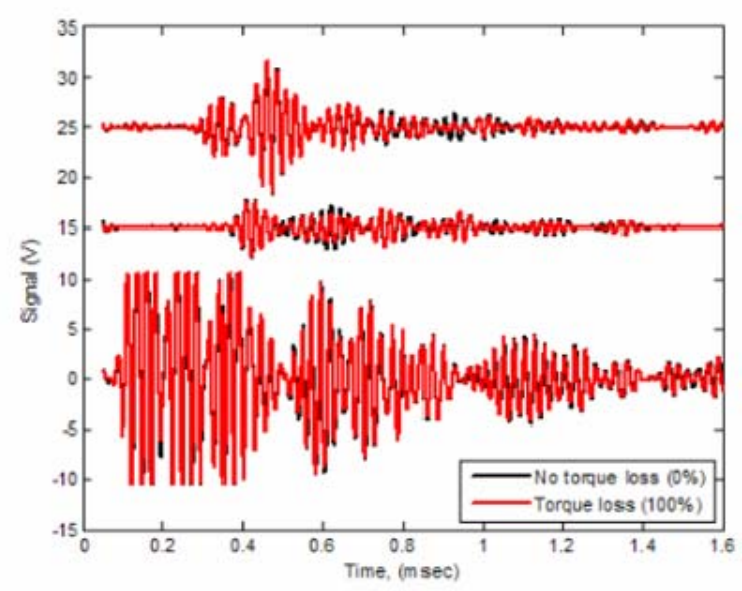

d) $100 \%$ torque loss

Figure 3-1-10. Sensing time response, B0 torque loss, E2 $40 \mathrm{kHz}$ excitation. 


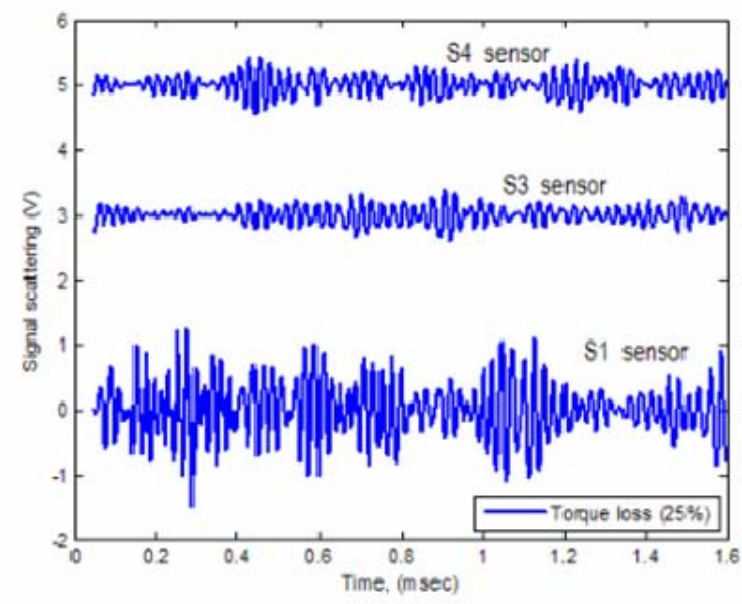

a) $25 \%$ torque loss

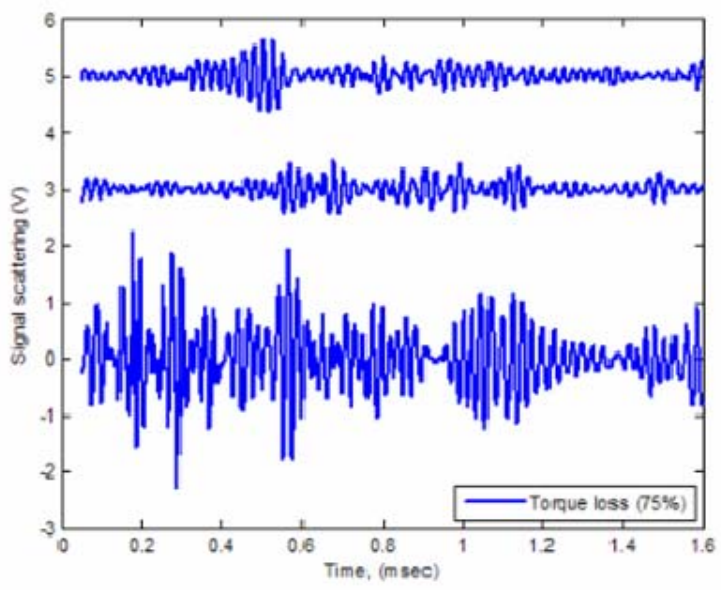

c) $75 \%$ torque loss

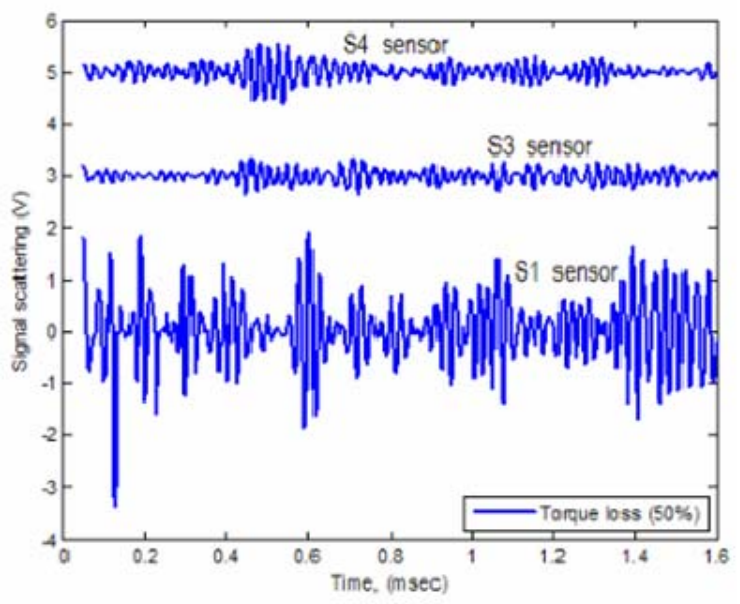

b) $50 \%$ torque loss

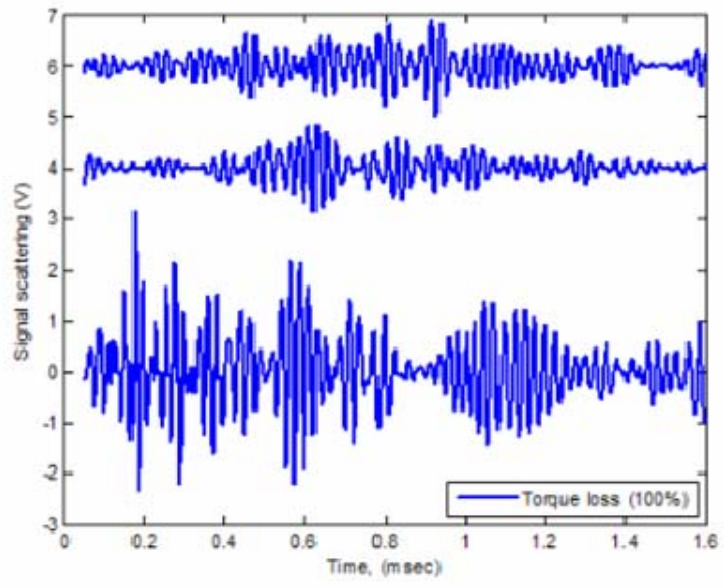

d) $100 \%$ torque loss

Figure 3-1-11. Scattering signals, B0 torque loss, E2 $40 \mathrm{kHz}$ excitation. 


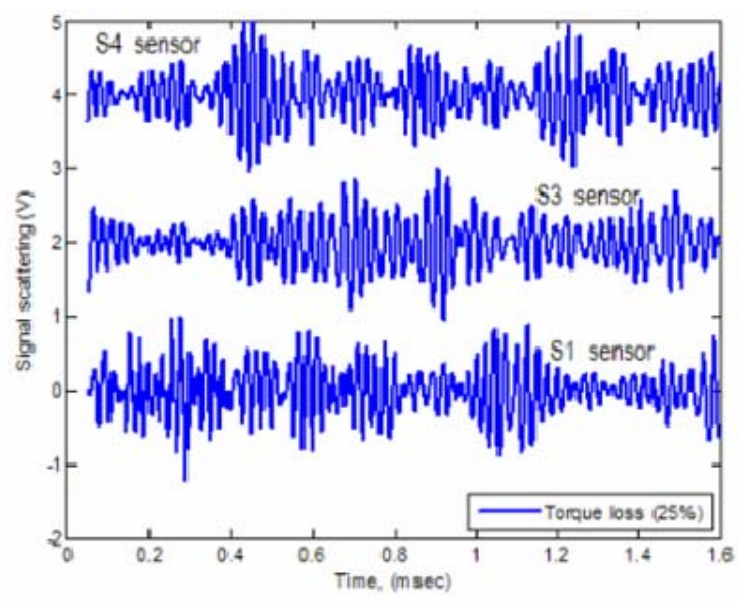

a) $25 \%$ torque loss

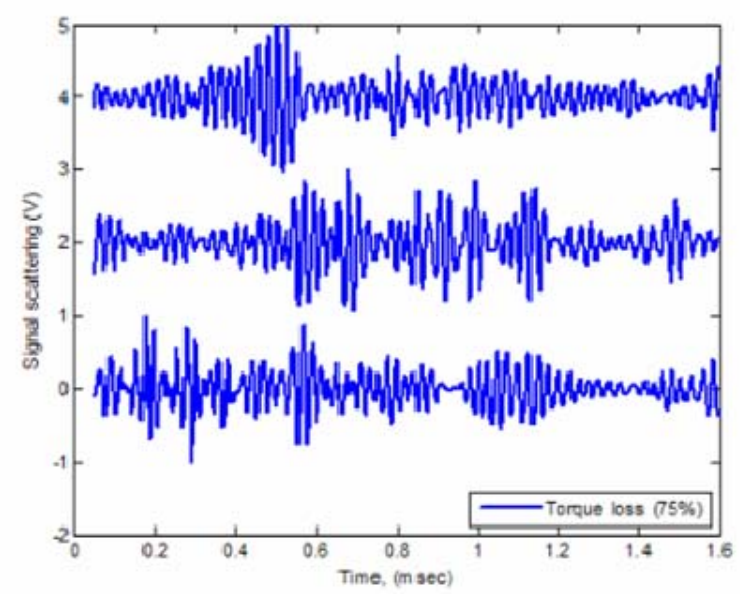

c) $75 \%$ torque loss

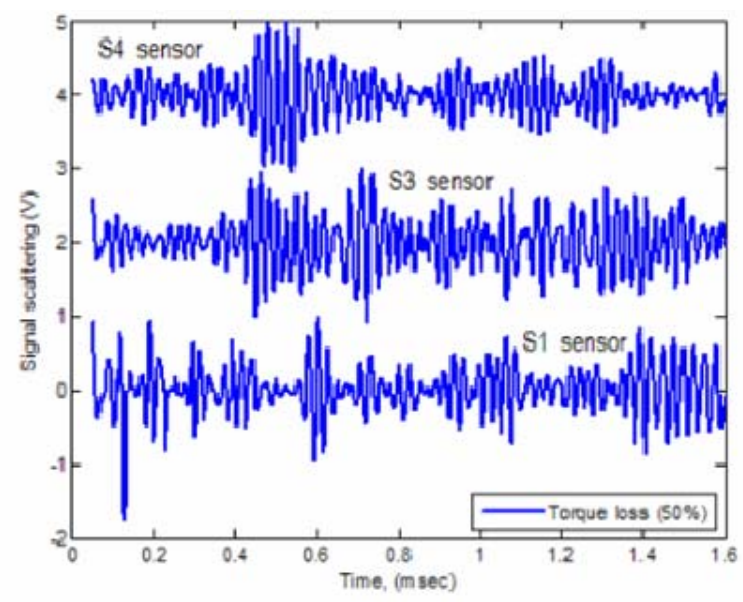

b) $50 \%$ torque loss

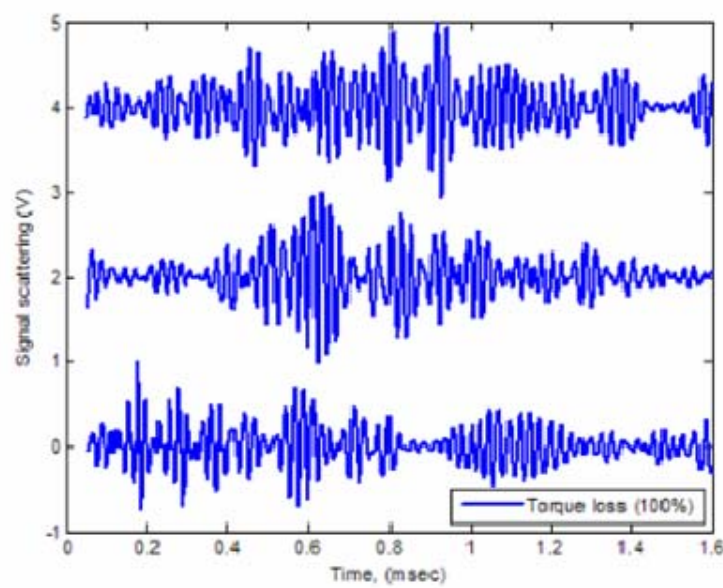

d) $100 \%$ torque loss

Figure 3-1-12. Normalized Scattering signals, B0 torque loss, E2 $40 \mathrm{kHz}$ excitation.

Figure 3-1-13 and Figure 3-1-14 present the damage index using the energy magnitudes due to frequency response $\left(D I_{f}\right)$ and using the signals in time $\left(D I_{t}\right)$ at $\mathrm{S} 1, \mathrm{~S} 3$ and S4 under E2 excitation. For each, the damage index is more sensitive to changes in damage level as compared to changes in excitation frequency. The damage detection capabilities of each sensor using time and frequency response magnitudes are favorable within the jointed composite panel due to Bolt 0 torque loss under the E2 excitation configuration. 


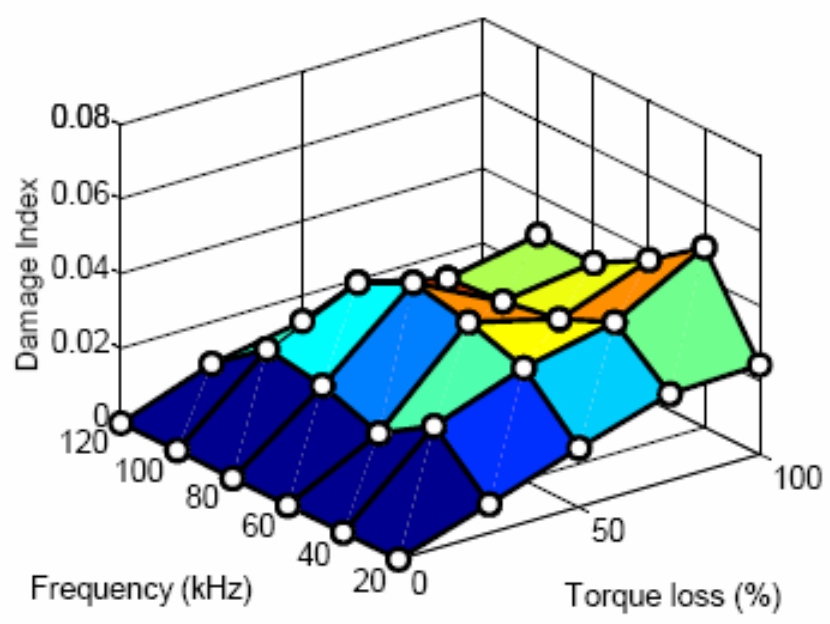

(a) S1 sensor

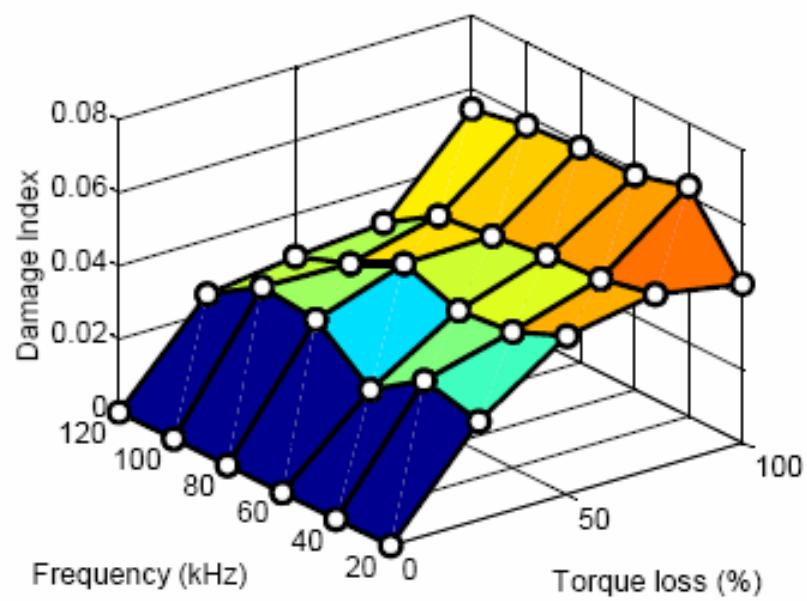

(b) S3 sensor

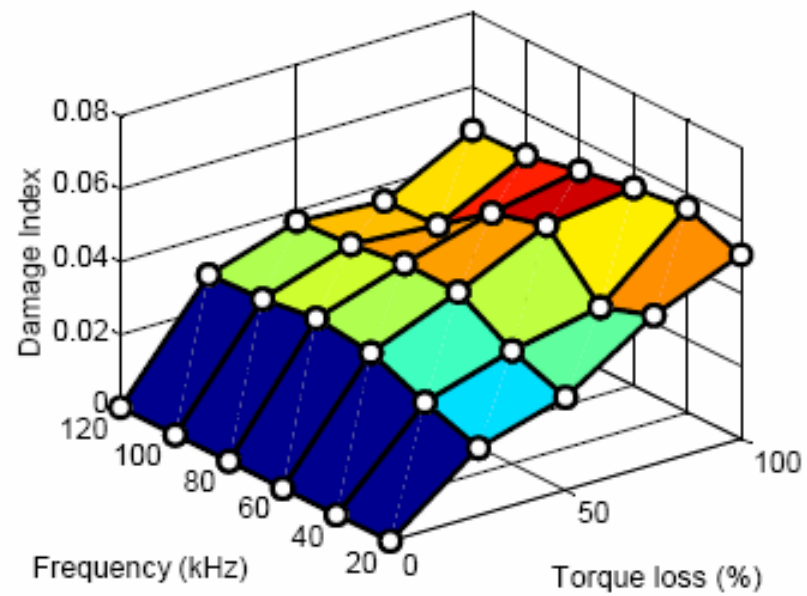

(c) S4 sensor

Figure 3-1-13. Damage index using the energy magnitudes in frequency vs. B0 torque loss and excitation frequency, E2 excitation. 


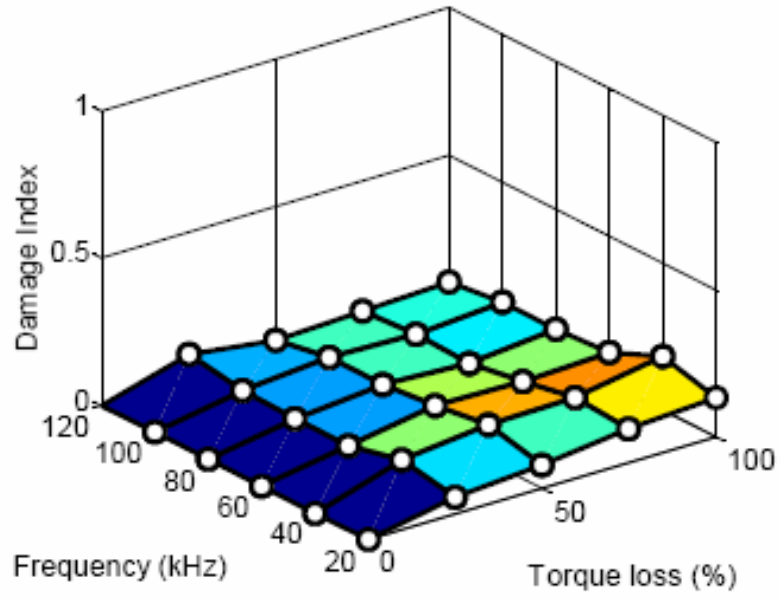

(a) $\mathrm{S} 1$ sensor

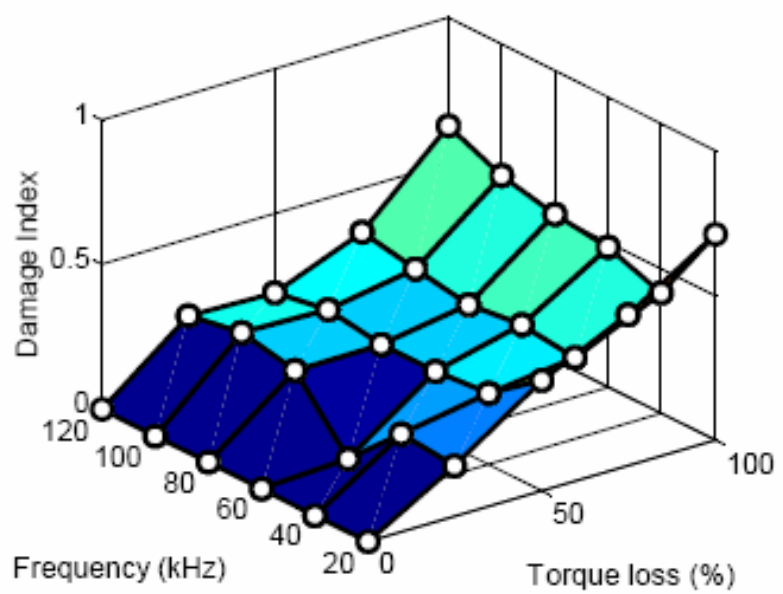

(b) S3 sensor

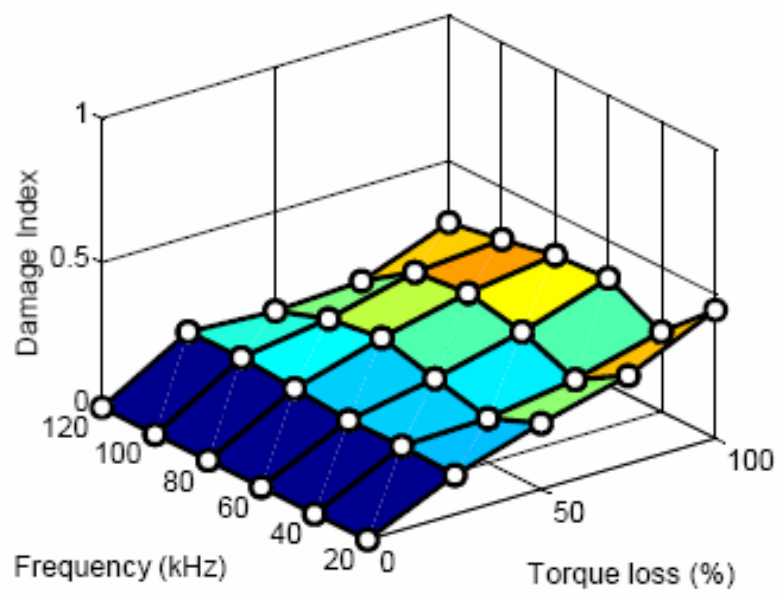

(c) $\mathrm{S} 4$ sensor

Figure 3-1-14. Damage index using signals in time in vs. B0 torque loss and excitation frequency, E2 excitation. 
Figure 3-1-14 displays smaller damage sensitivity in S1 as compared to the damage sensitivity in S3 and S4 sensors. Such a trend was displayed in the S2 sensor under E1 excitation, as noted in the previous section.

Figure $3-1-15$ presents the normalized average damage index $\left(D I_{\text {average }}\right)$ of the jointed composite panel due to torque loss in Bolt 0 for E2 excitation. The trend indicates an immediate increase in damage index from the undamaged scenario to the scenario of smallest damage, a torque loss of $25 \%(3.75 \mathrm{~N}-\mathrm{m})$. The damage index as a damage detection method is capable of indicating such small levels of torque loss within the composite joint specimen. The trend line due to S1 sensor is lower than the trend lines due to the S3 and S4 sensors. Similar to the S2 trend line in Figure 3-1-7, this indicates S1 sensor's decreased ability to perceive damage within the composite joint as compared to the S3 and S4 sensors. The magnitude of average damage index from the S3 sensor is higher than the damage index from S4 at each torque loss value. This presents the idea that, under E2 excitation, the sensor in Quadrant 3 indicates a higher incidence of damage than the sensor in Quadrant 4. 


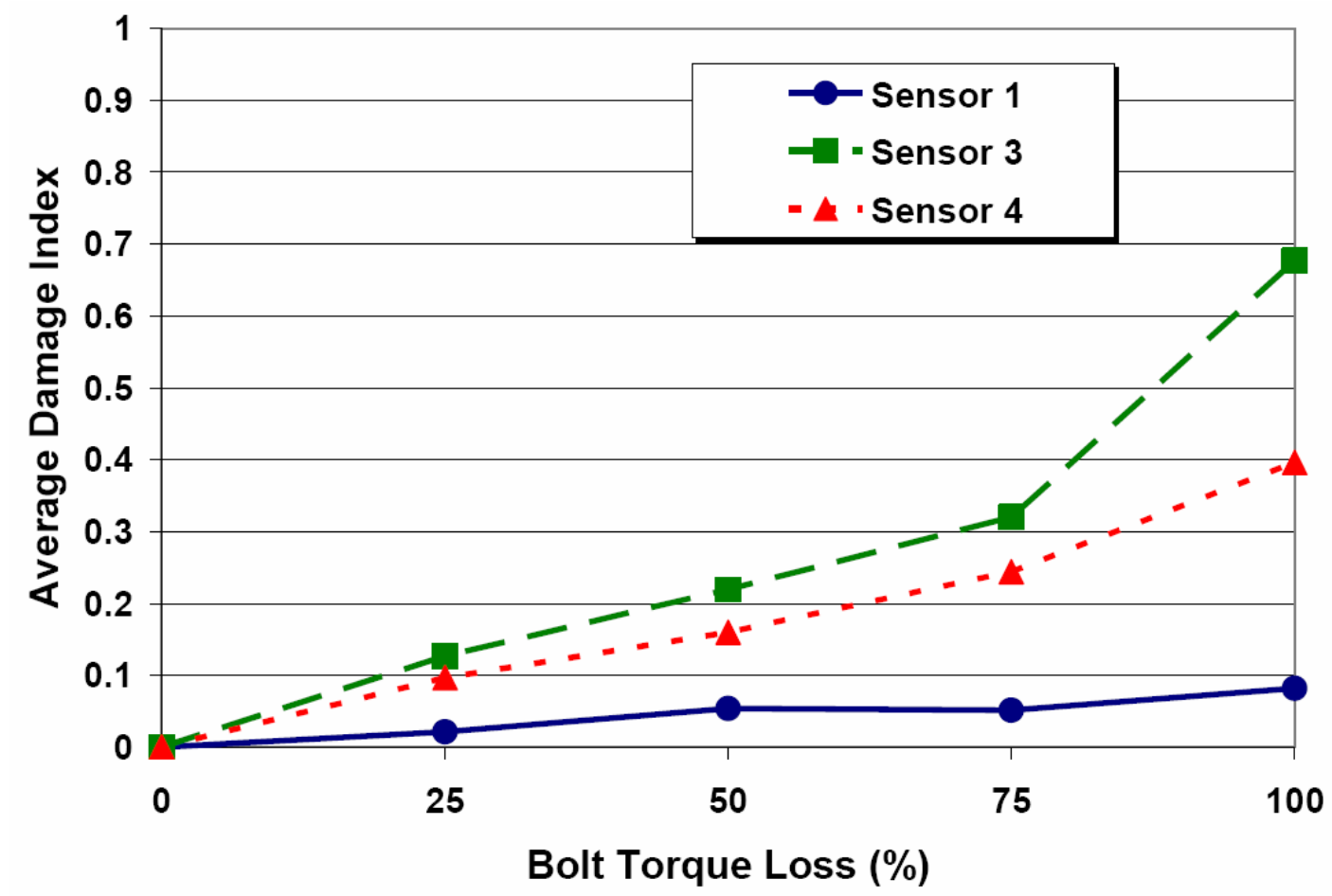

Figure 3-1-15. Average damage index, B0 torque loss, E2 excitation.

Figure 3-1-16 presents the total damage index under E2 excitation with respect to Bolt 0 torque loss. The total damage index contribution from the S3 and S4 sensors across the joint makes up nearly the entire damage index contribution from all three sensors, S1, S3 and S4. As noted in Figure 3-1-8, this further indicates the importance of sensing capability of sensors across the composite joint when damage is due to bolt torque loss within the joint. 


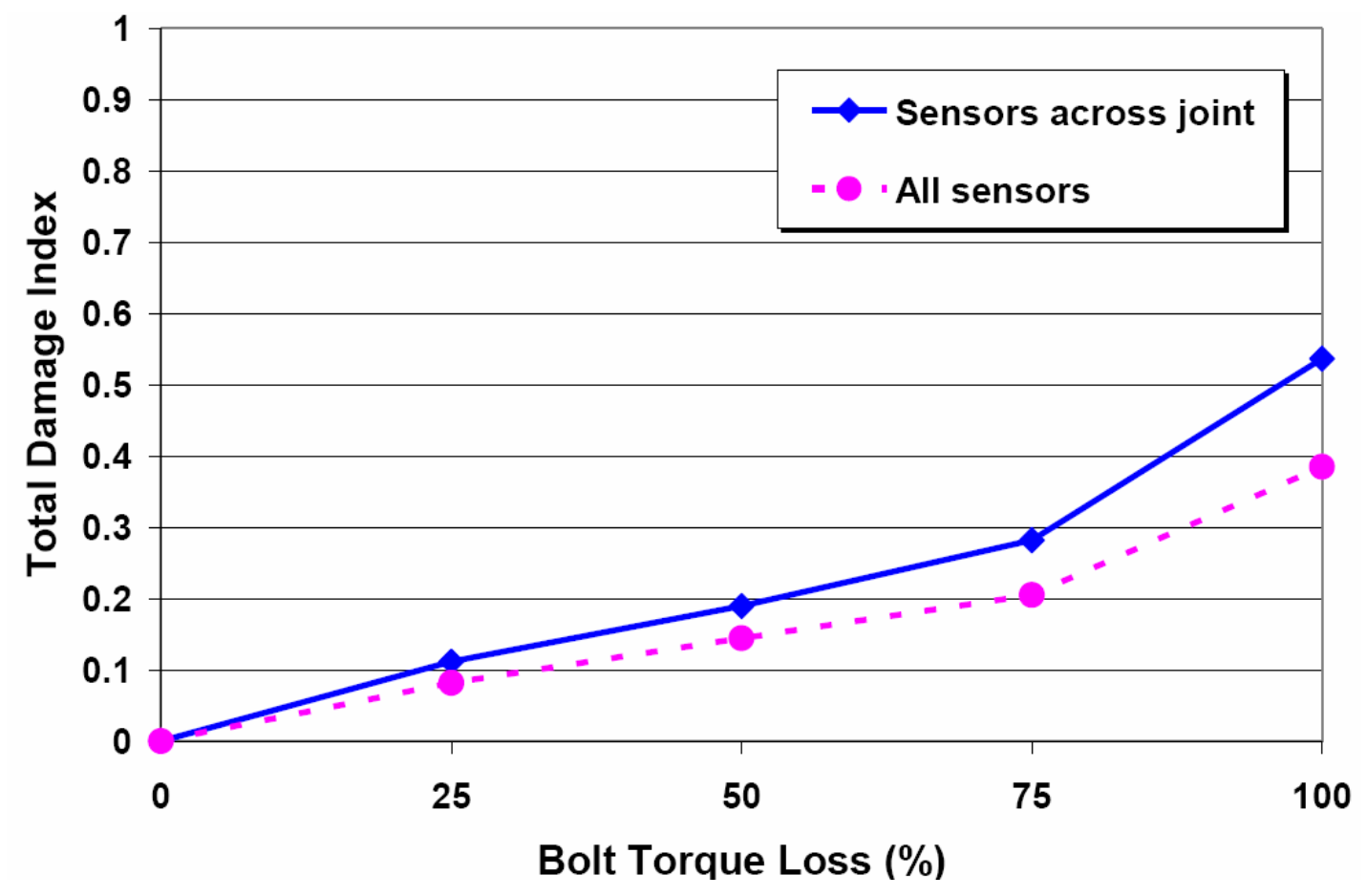

Figure 3-1-16. Total damage index, B0 torque loss, E2 excitation.

\subsubsection{Bolt 0, Excitation Case III}

Using the piezo-patch in Quadrant 3 (E3) for excitation of the jointed composite panel, sensing information is compiled at the three remaining sensors (S1, S2, S4) located within the remaining three quadrants. In this excitation case, the actuation occurs in the same quadrant as the present damage due to torque loss. Figure 3-1-17 displays the excitation and sensing configuration and indicates the Bolt 0 location of simulated damage. Figure 3-1-18 represents the time response signals at S1, S2 and S4 sensors with respect to Bolt 0 torque loss at a $40 \mathrm{kHz}$ excitation frequency. 


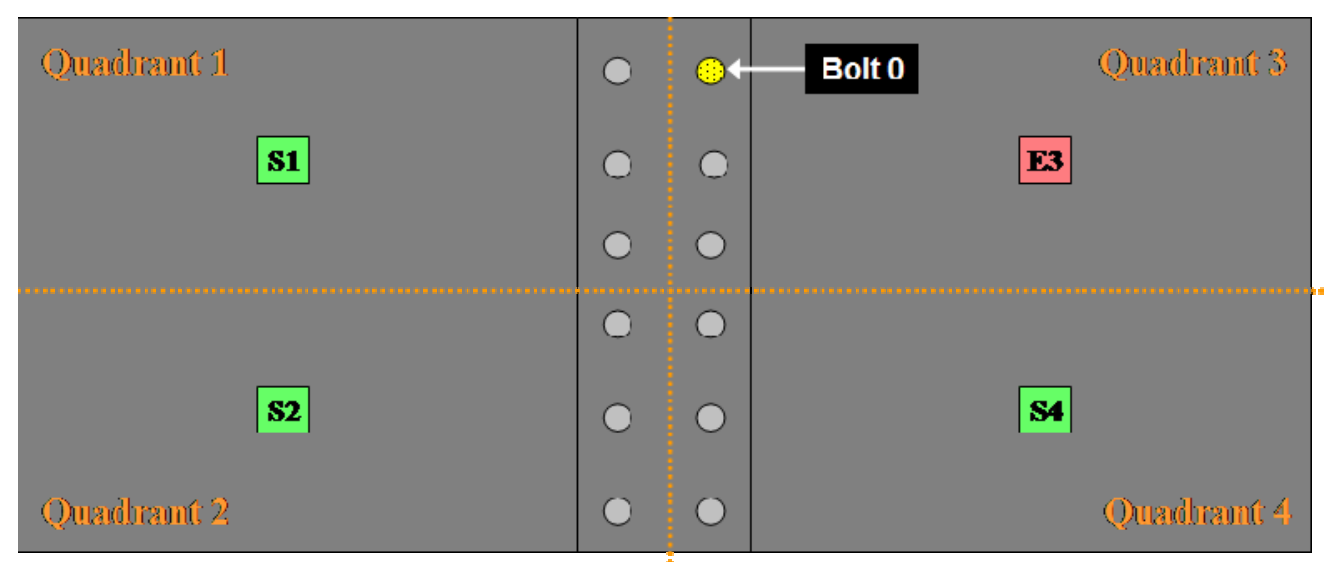

Figure 3-1-17. Excitation Case III, variable Bolt 0 torque loss.

As shown in Figure 3-1-18, the magnitude of the time response signal from the S4 sensor location is noticeably greater than the sensor time response signal at locations S1 and S2. This higher magnitude occurs due to the sensor's shared panel location as the actuator, as noted similarly in the Case I configuration with S2 and in the Case II configuration with S1. The comparably smaller S1 and S2 time response signals are located across the joint on composite 1 , further emphasizing the incident signal loss after passing through the composite joint.

Figure 3-1-19 shows the scattering signal for a $40 \mathrm{kHz}$ excitation frequency under E3 actuation. The same scattering signal is normalized and shown in Figure 3-1-20. The signal scattering at S1 and S2 sensors increase in magnitude as torque loss increases. 


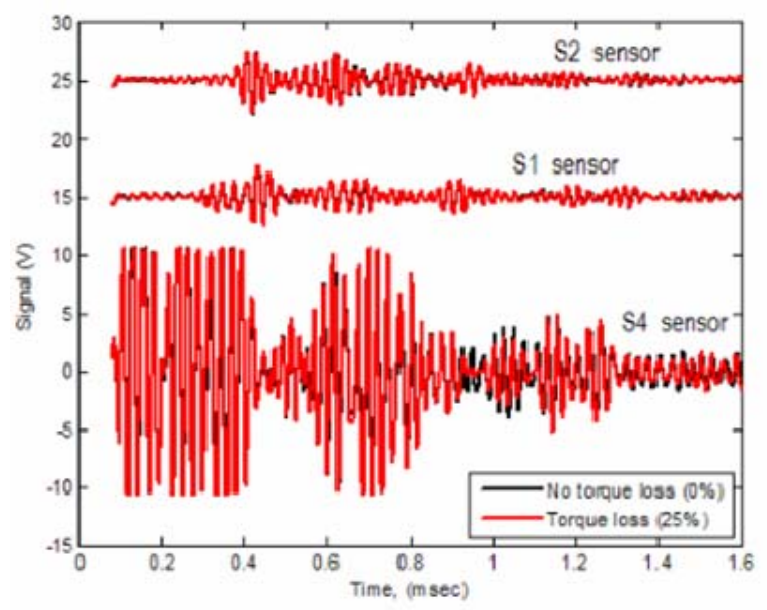

a) $25 \%$ torque loss

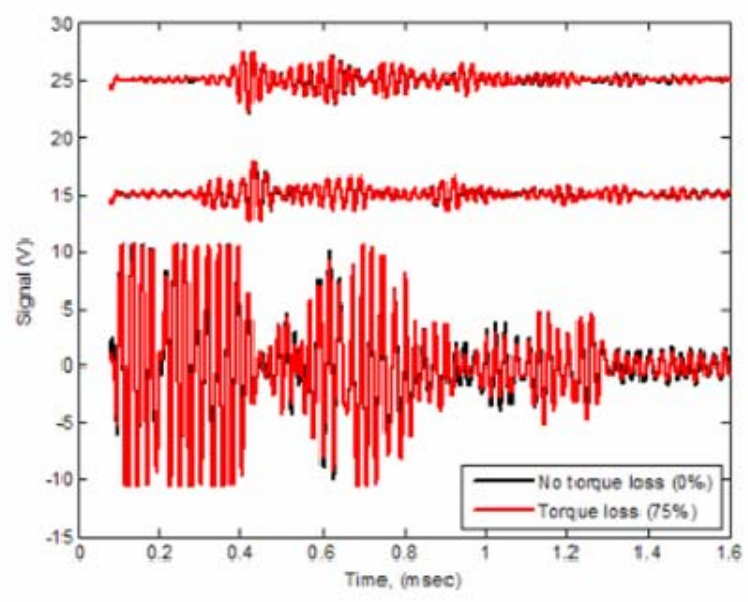

c) $75 \%$ torque loss

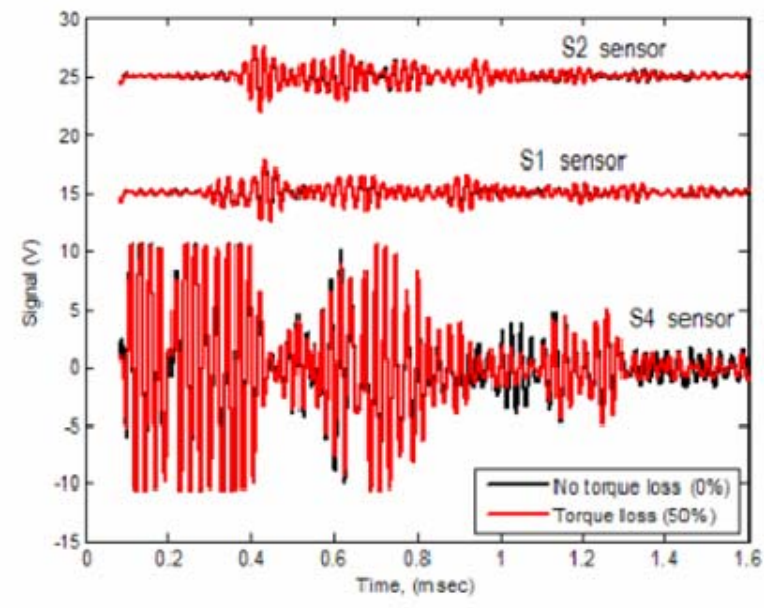

b) $50 \%$ torque loss

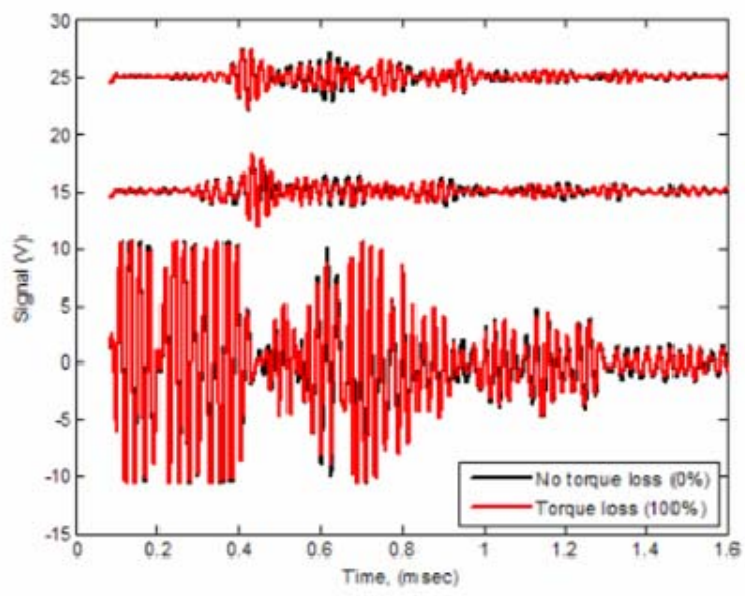

d) $100 \%$ torque loss

Figure 3-1-18. Sensing time response, B0 torque loss, E3 $40 \mathrm{kHz}$ excitation. 


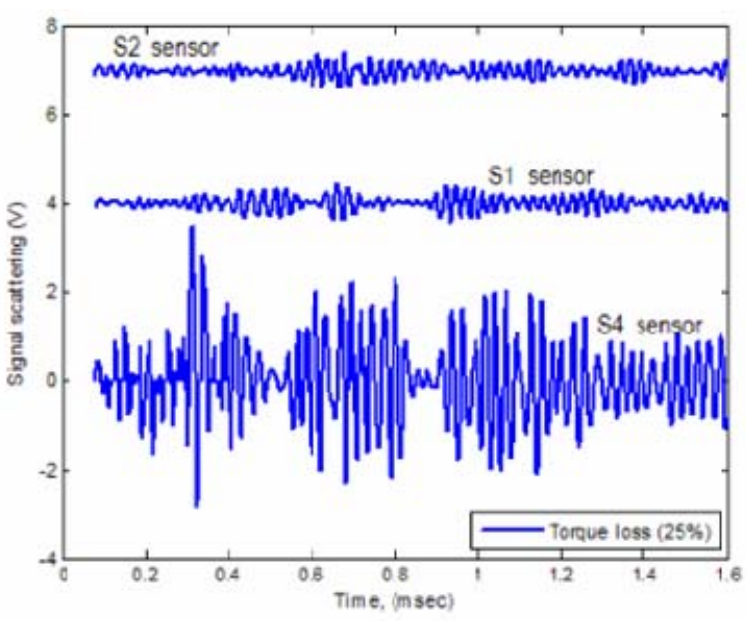

a) $25 \%$ tontue loss

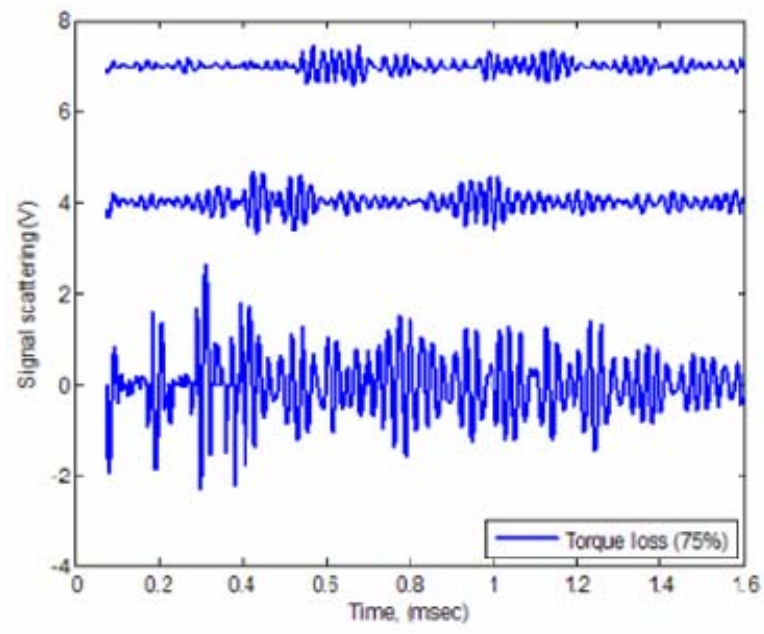

c) $75 \%$ torque loss

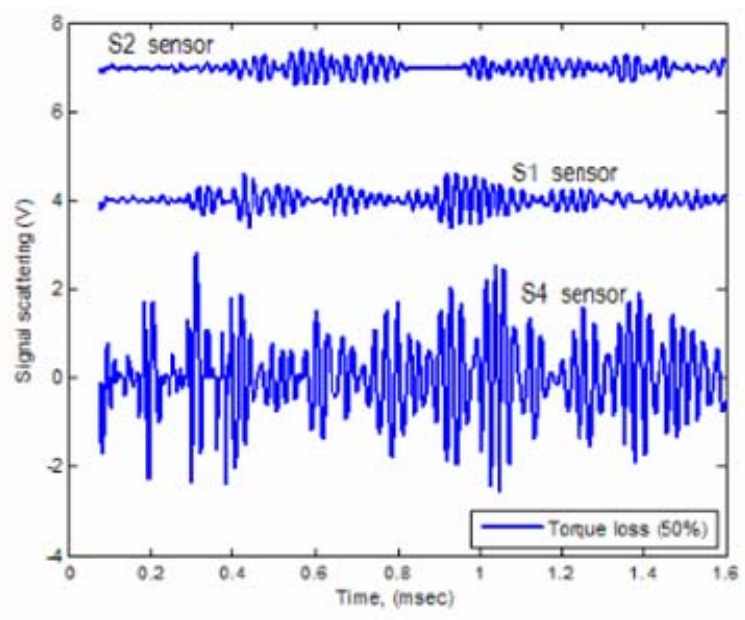

b) $50 \%$ torywe loss

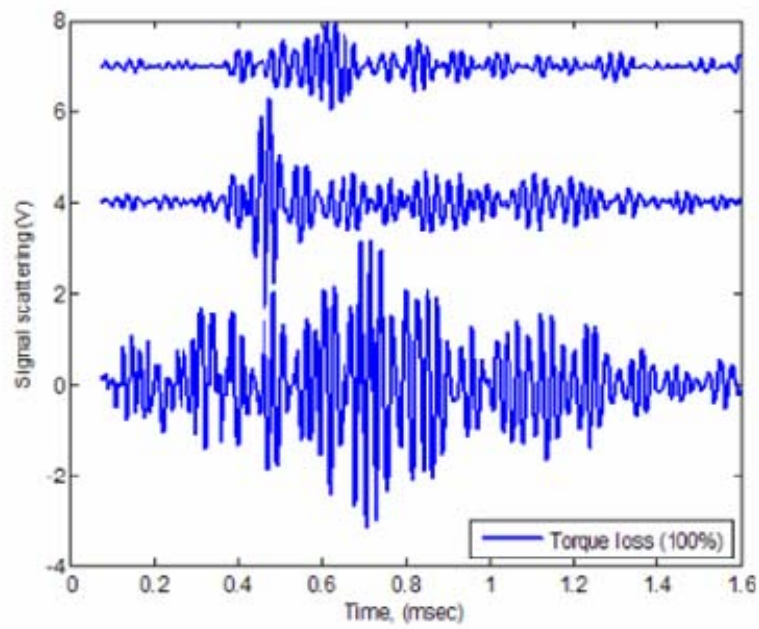

d) $100 \%$ torque loss

Figure 3-1-19. Scattering signals, B0 torque loss, E3 $40 \mathrm{kHz}$ excitation. 


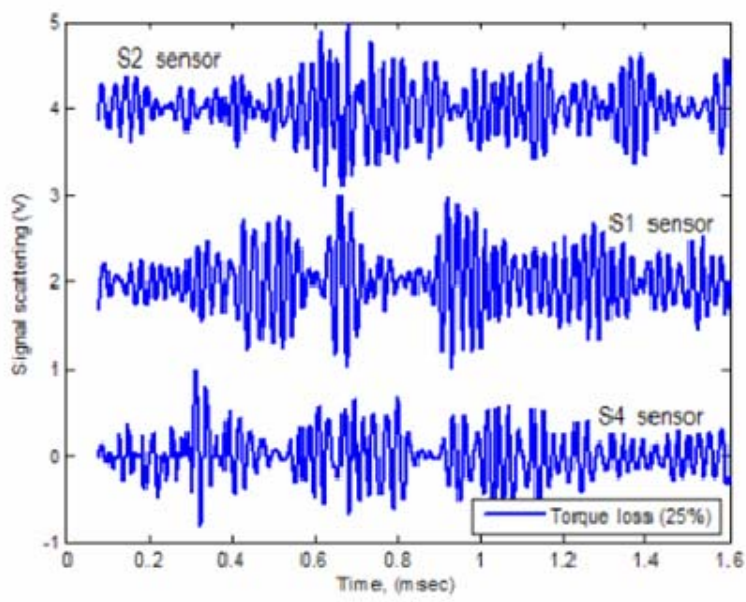

a) $25 \%$ torque loss

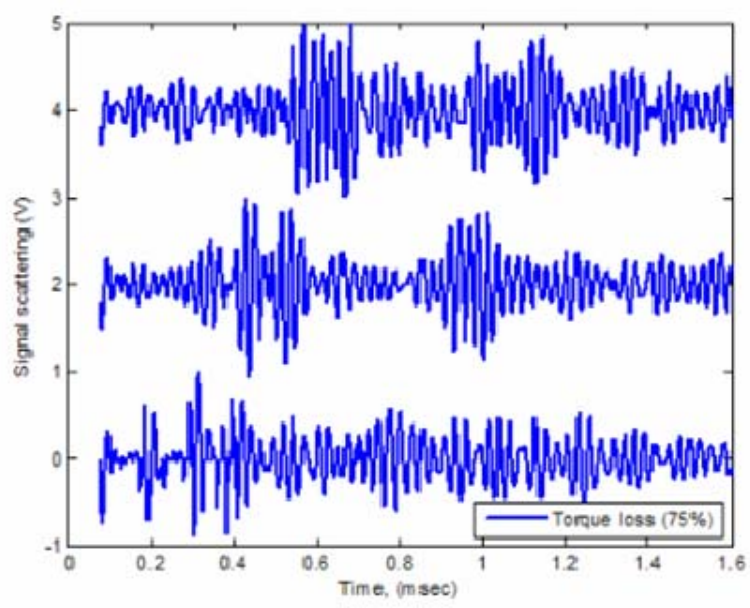

c) $75 \%$ torque loss

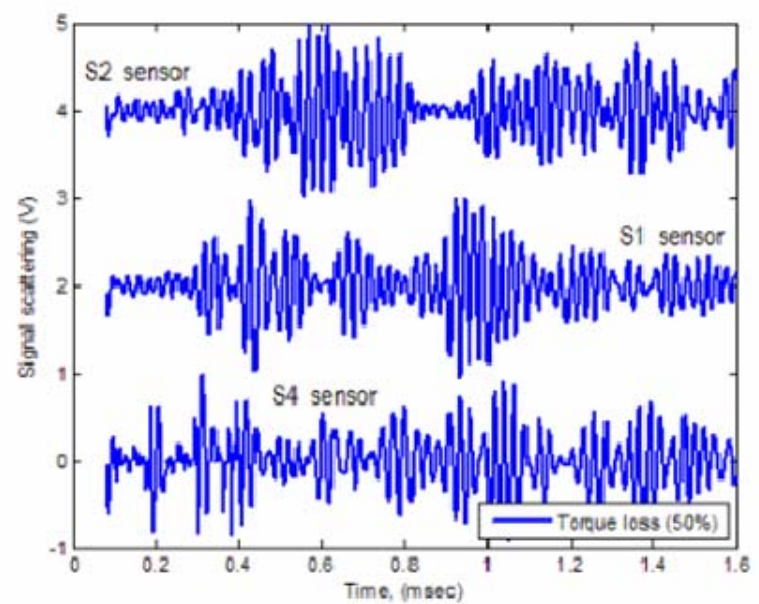

b) $50 \%$ torque loss

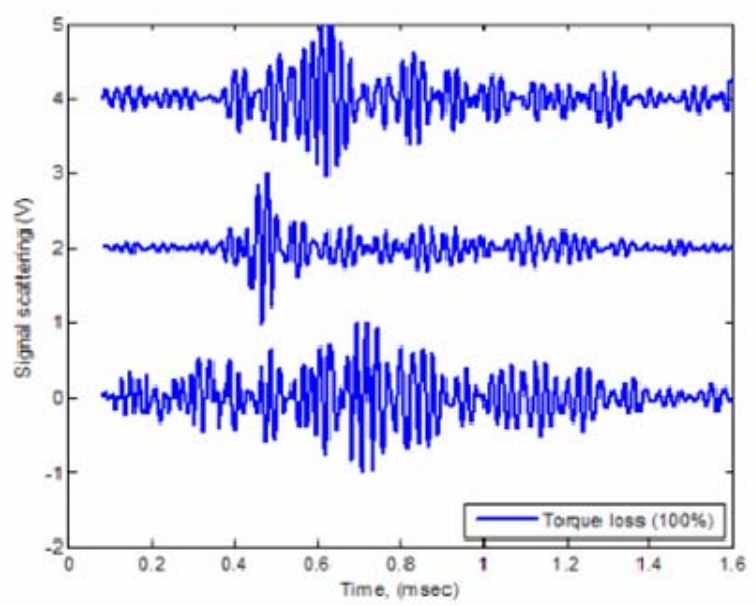

d) $100 \%$ torque loss

Figure 3-1-20. Normalized scattering signals, B0 torque loss, E3 $40 \mathrm{kHz}$ excitation.

Figure 3-1-21 and Figure 3-1-22 present the damage index using the energy magnitudes due to frequency response $\left(D I_{f}\right)$ and using the signals in time $\left(D I_{t}\right)$ at $\mathrm{S} 1, \mathrm{~S} 2$ and S4 under E3 excitation. Even when actuation is performed in the same quadrant where damage is present due to torque loss, the damage detection capabilities of each sensor using time and frequency response magnitudes are favorable under the E3 excitation configuration with respect to Bolt 0 torque loss. 


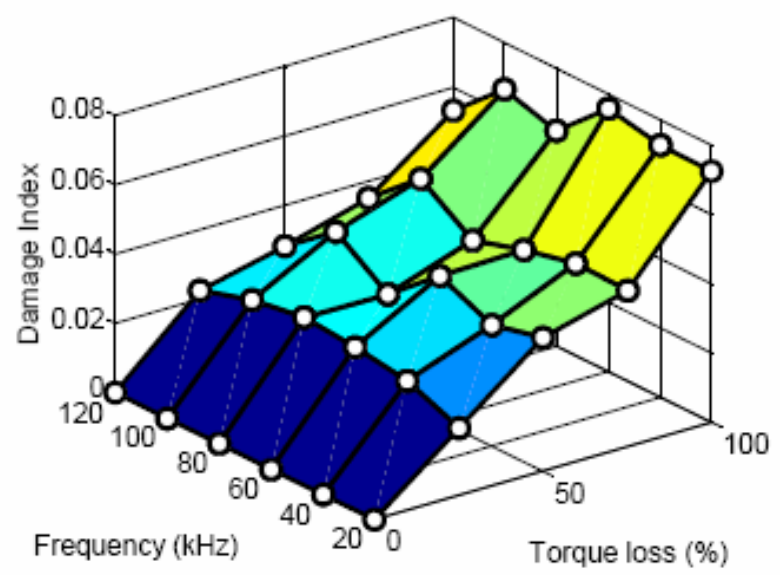

(a) S1 sensor

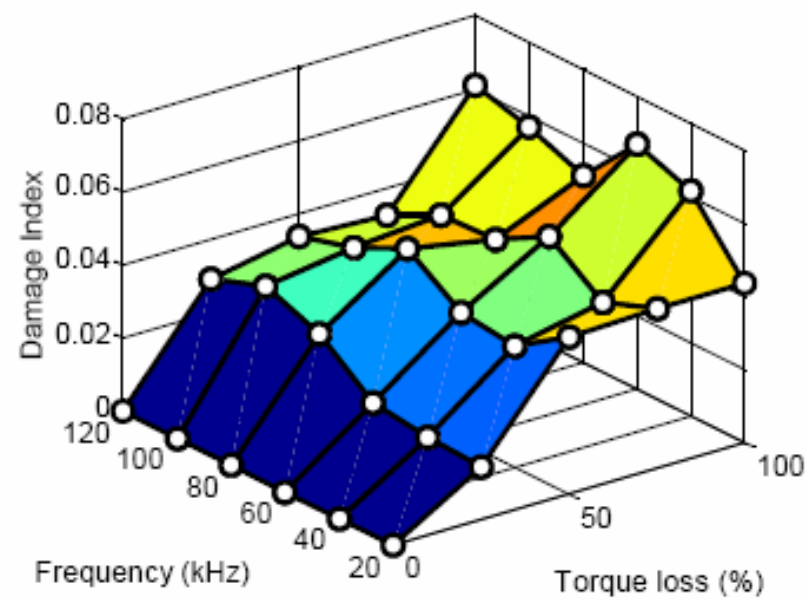

(b) S2 sensor

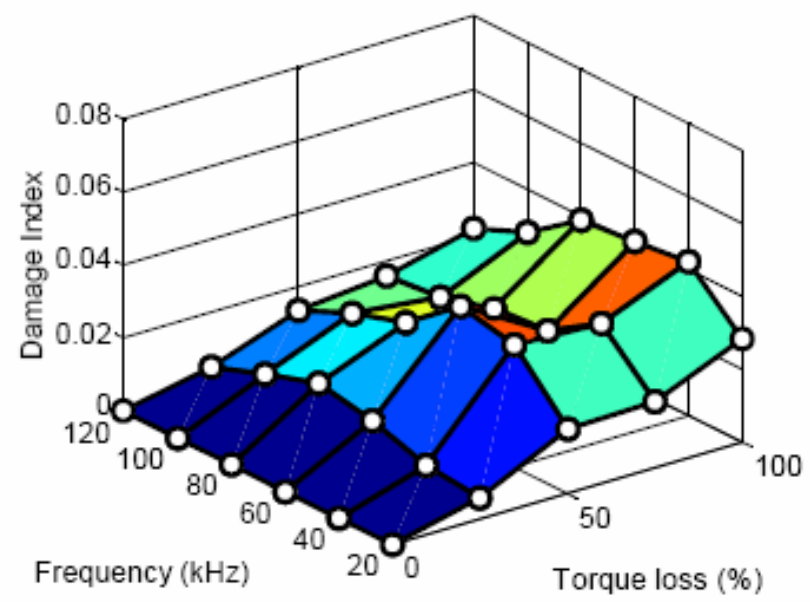

(c) S4 sensor

Figure 3-1-21. Damage index using the energy magnitudes in frequency vs. B0 torque loss and excitation frequency, E3 excitation. 


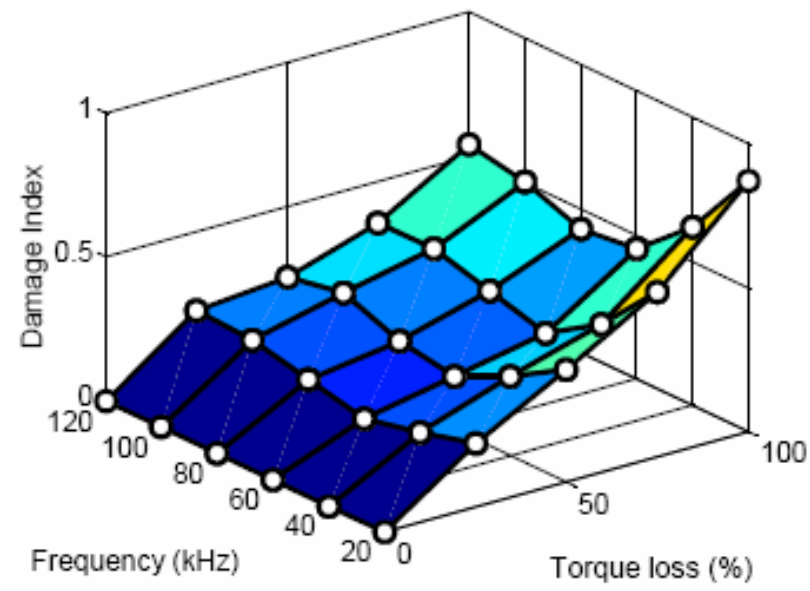

(a) $\mathrm{S} 1$ sensor

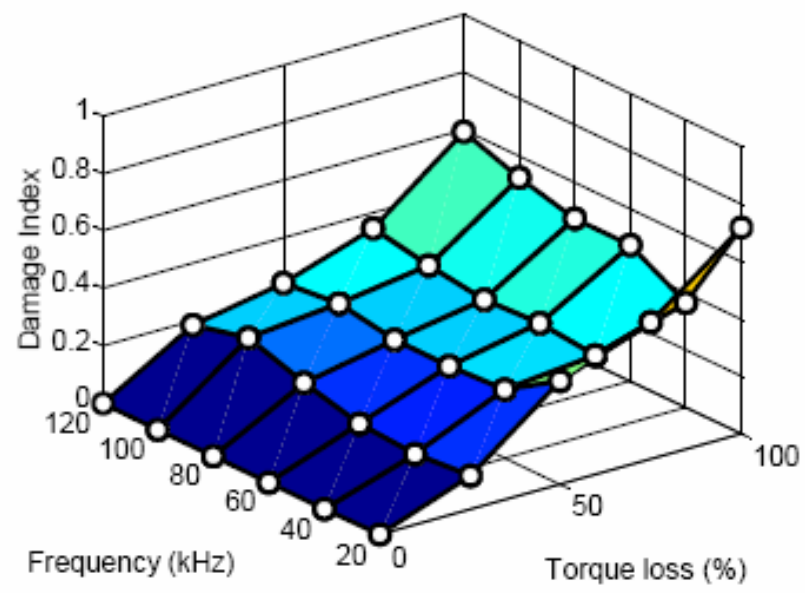

(b) S2 sensor

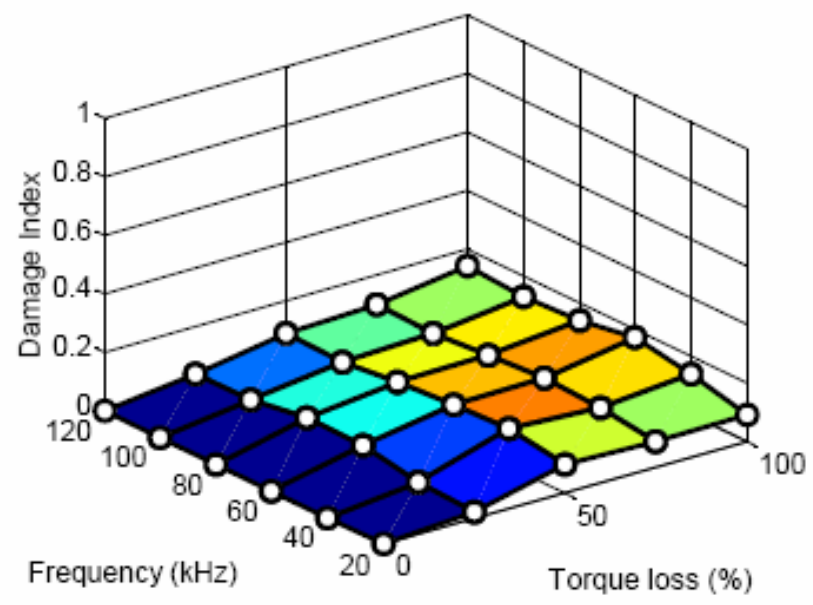

(c) S4 sensor

Figure 3-1-22. Damage index using the signals in time vs. B0 torque loss and excitation frequency, E3 excitation. 
Figure 3-1-22 displays smaller damage sensitivity in S4 as compared to the damage sensitivity in S1 and S2 sensors. Such a trend was displayed in the S2 sensor under E1 excitation, and in the S1 sensor under E2 excitation, as noted in the previous sections. This reiterates the smaller capacity for damage detection within sensors that are located on the same composite panel as frequency actuation.

Figure 3-1-23 presents the normalized average damage index $\left(D I_{\text {average }}\right)$ of the jointed composite panel due to torque loss in Bolt 0 for E3 excitation. As in E1 and E2 excitation cases, the damage index trend-lines of all three sensors indicates an immediate increase in damage index from the undamaged scenario to the scenario of $25 \%$ torque loss. The damage index as a method for damage detection is capable of indicating such small levels of torque loss within the composite jointed panel. The trend line due to S4 sensor is lower than the trend lines due to the S1 and S2 sensors. Similar to the S2 trend line in Figure 3-1-7 and the S1 trend in Figure 3-1-15, this indicates the S4 sensor's decreased ability to perceive damage within the composite joint as compared to the S1 and S2 sensors. 


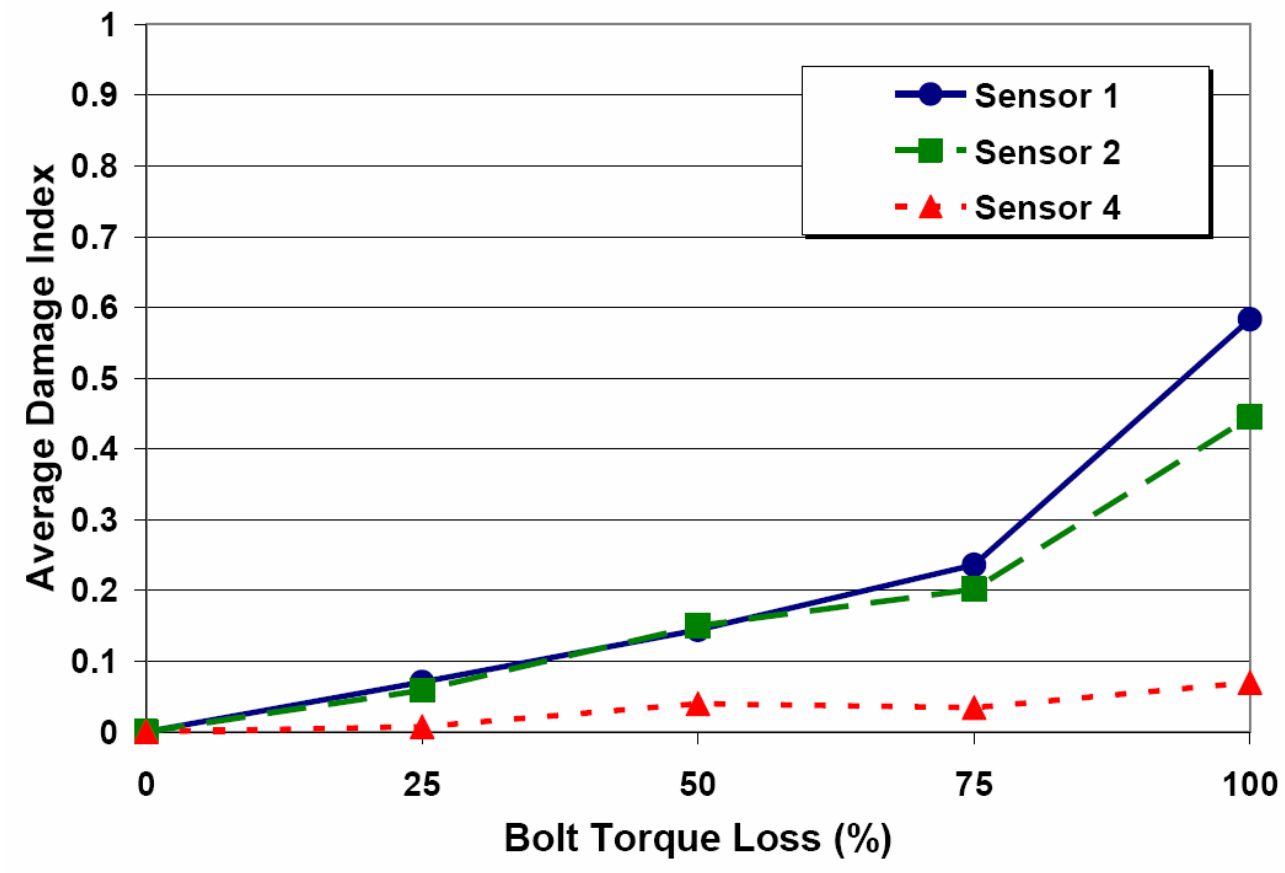

Figure 3-1-23. Average damage index, B0 torque loss, E3 excitation.

In Figure 3-1-23, the magnitude of average damage index from the $\mathrm{S} 1$ sensor is nearly indistinguishable from the values associated with $\mathrm{S} 1$ at torque loss values below $75 \%$. This presents the idea that, under E3 excitation, it is difficult to distinguish whether the sensing capability at S1 or S2 provides more insight into damage detection in the composite jointed specimen.

Figure 3-1-24 presents the total damage index under E3 excitation with respect to Bolt 0 torque loss. The total damage index contribution from the S1 and S2 sensors across the joint makes up nearly the entire damage index contribution from all three sensors, S1, S2 and S4. As noted in Figure 3-1-8 and Figure 3-1-16, this further indicates the importance of sensing capability of sensors across the composite joint when damage is located within the joint. 


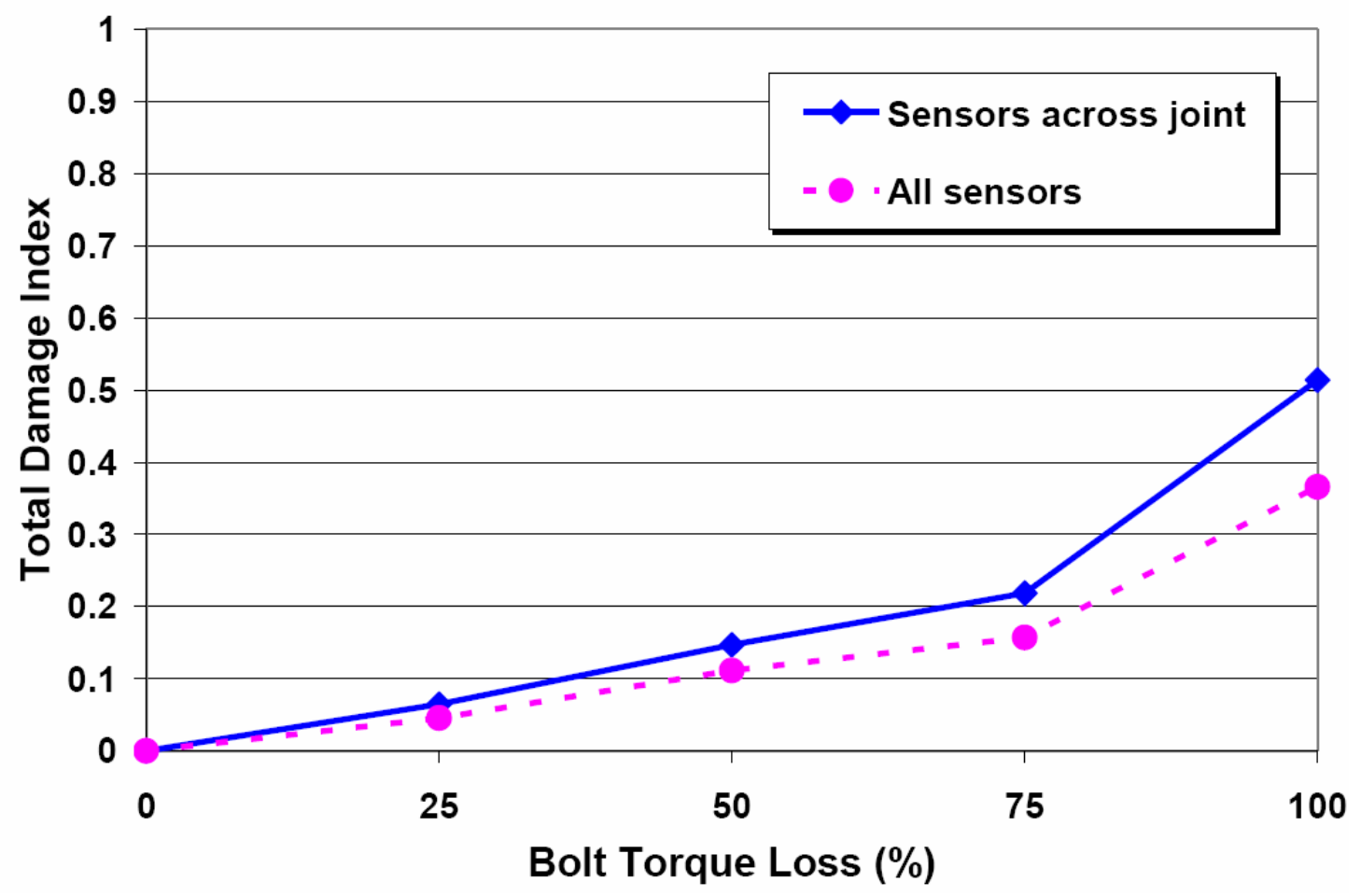

Figure 3-1-24. Total damage index, B0 torque loss, E3 excitation.

\subsubsection{Bolt 0, Excitation Case IV}

Using the piezo-patch in Quadrant 4 (E4) for excitation of the jointed composite panel, sensing information is compiled at the three remaining sensors (S1, S2, S3) located within the remaining three quadrants. In this excitation case, the actuation occurs on the same composite panel (composite 2) as the present damage due to torque loss, but in a different quadrant. Figure 3-1-25 displays the excitation and sensing configuration and indicates the Bolt 0 location of damage due to torque loss. Figure 3-1-26 shows the time response signals at S1, S2 and S3 sensors with respect to Bolt 0 torque loss at a $40 \mathrm{kHz}$ excitation frequency. 


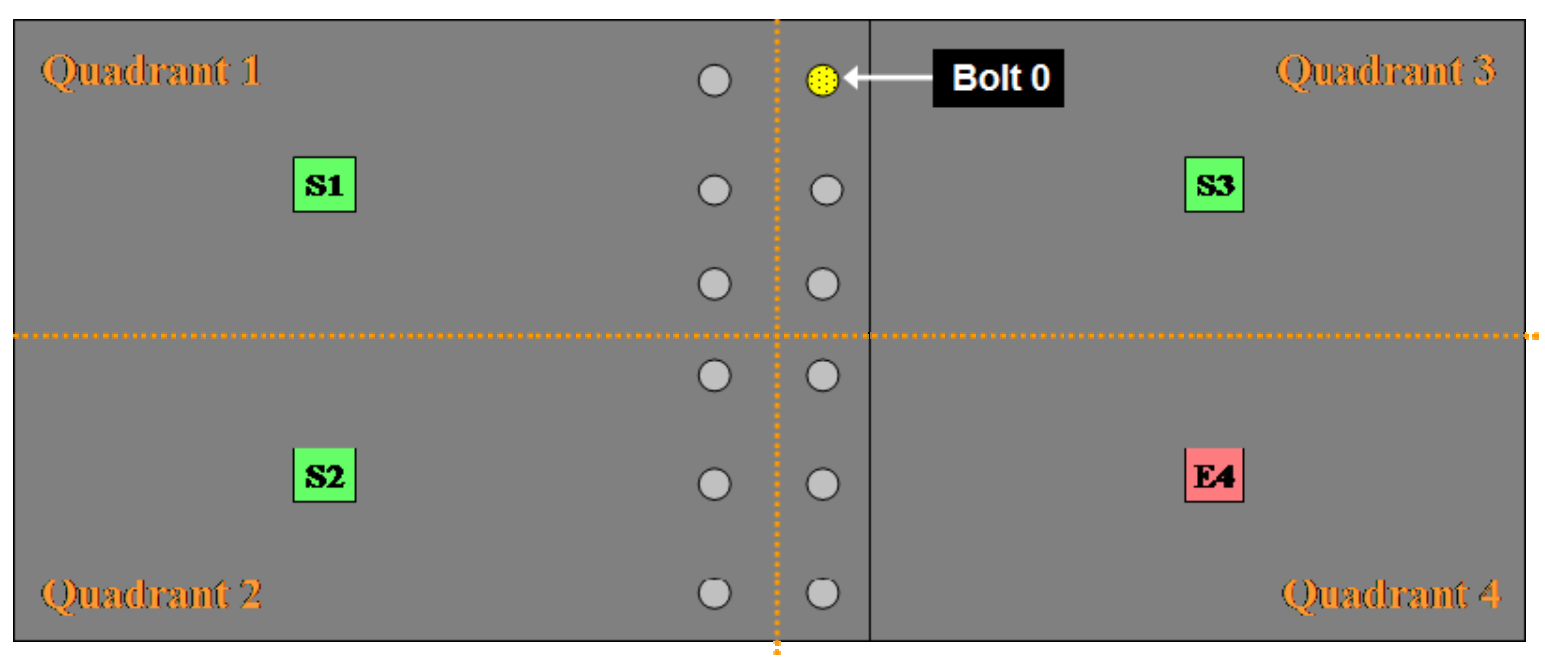

Figure 3-1-25. Excitation Case IV, variable Bolt 0 torque loss.

As shown in Figure 3-1-26, the magnitude of the time response signal from the S3 sensor location is noticeably greater than the sensor time response signal at locations S1 and S2. This higher magnitude occurs due to the sensor's shared panel location as the actuator, as noted similarly in the Case III configuration with S4. The signal loss from E4 over the joint to S1 and S2 is evident in the comparably smaller S1 and S2 time response signals

Figure 3-1-27 shows the scattering signal for a $40 \mathrm{kHz}$ excitation frequency under E4 actuation. The same scattering signal is normalized and shown in Figure 3-1-28. The signal scattering at S1 and S2 sensors increase in magnitude as torque loss increases. 


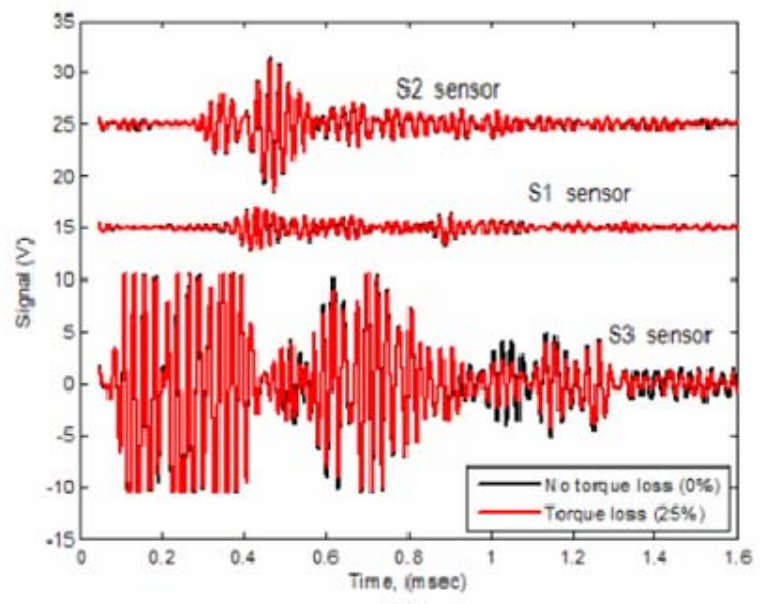

a) $25 \%$ torque loss

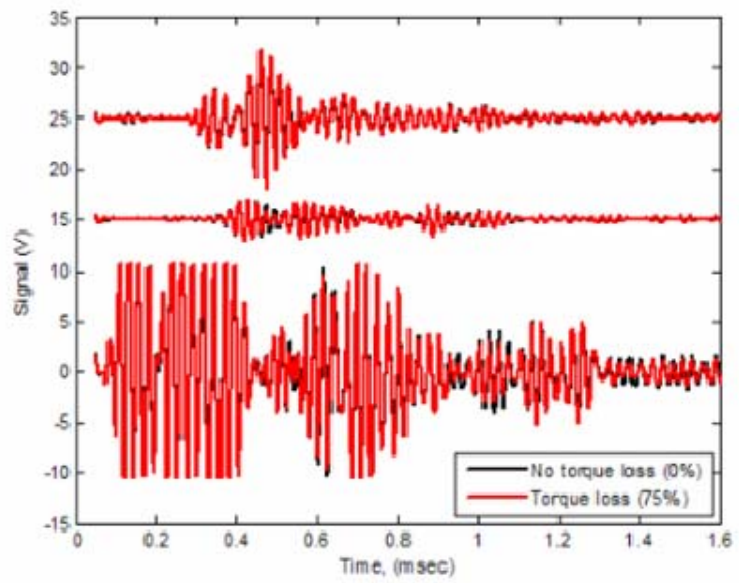

c) $75 \%$ torque loss

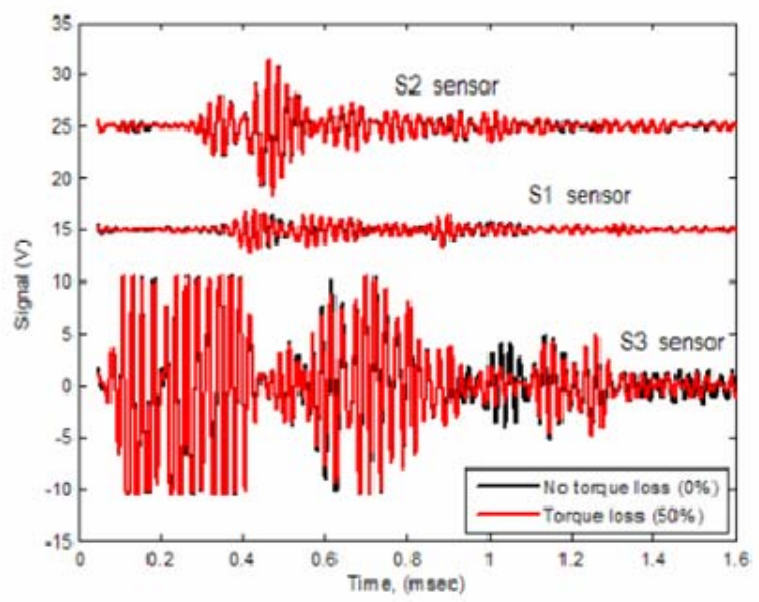

b) $50 \%$ torque loss

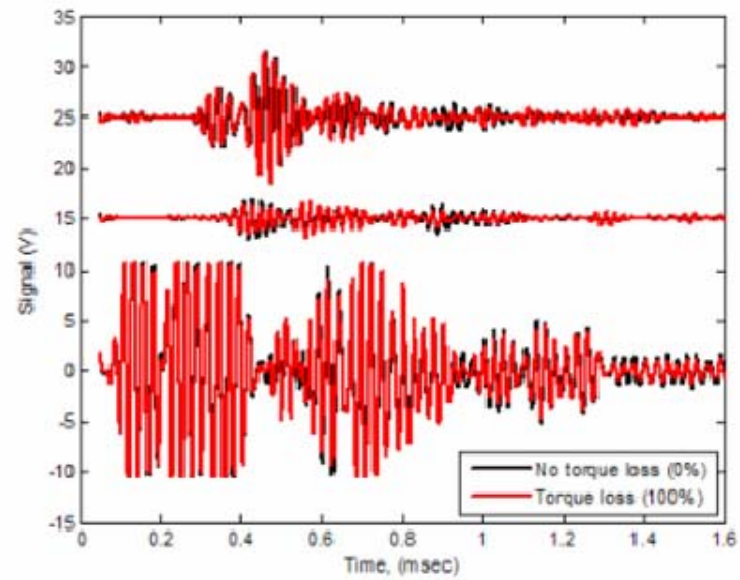

d) $100 \%$ tonque loss

Figure 3-1-26. Sensing time response, B0 torque loss, E4 $40 \mathrm{kHz}$ excitation. 


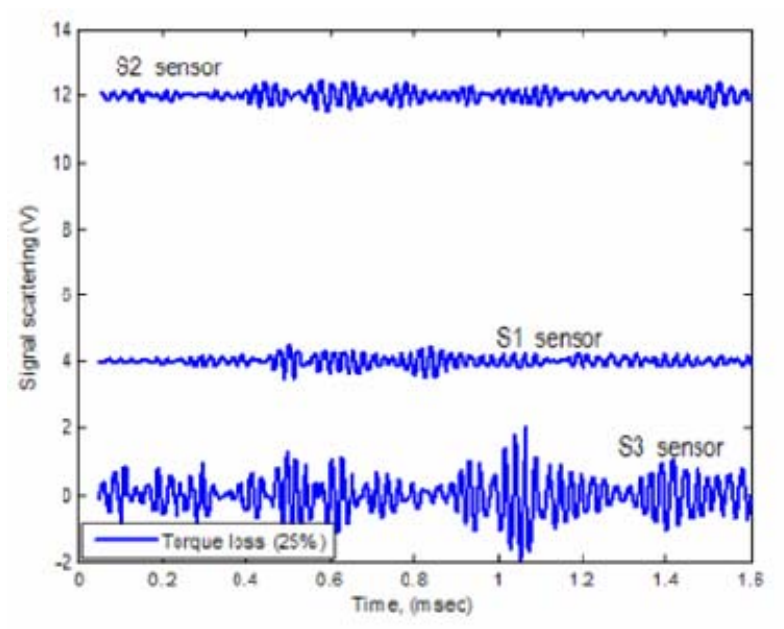

a) $25 \%$ torque loss

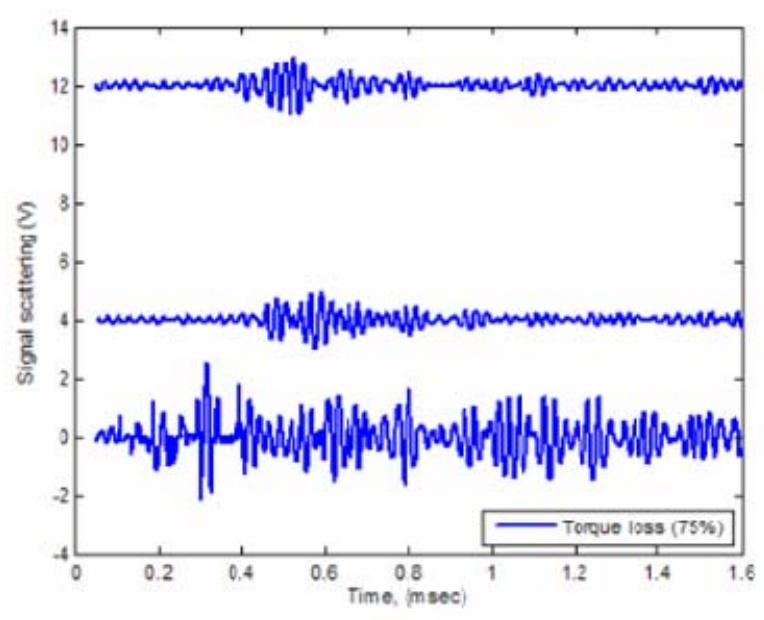

c) $75 \%$ torque loss

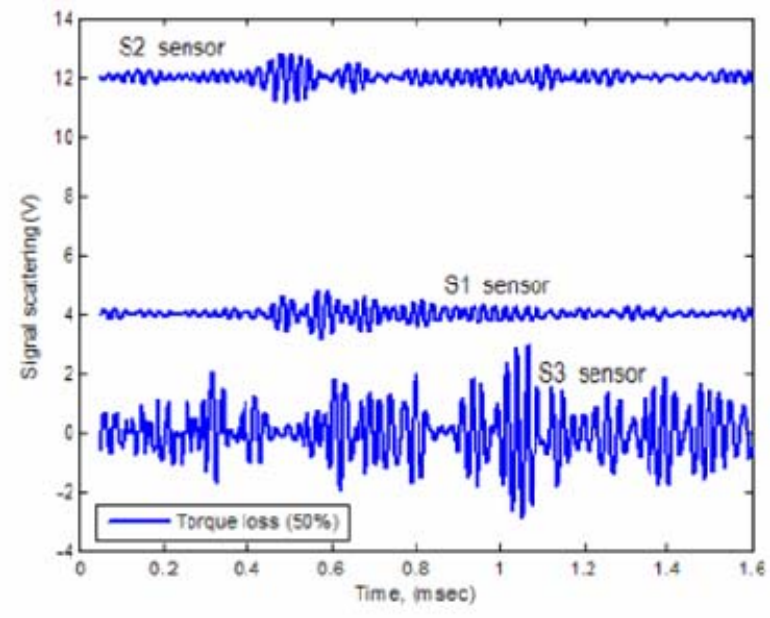

b) $50 \%$ tornue loss

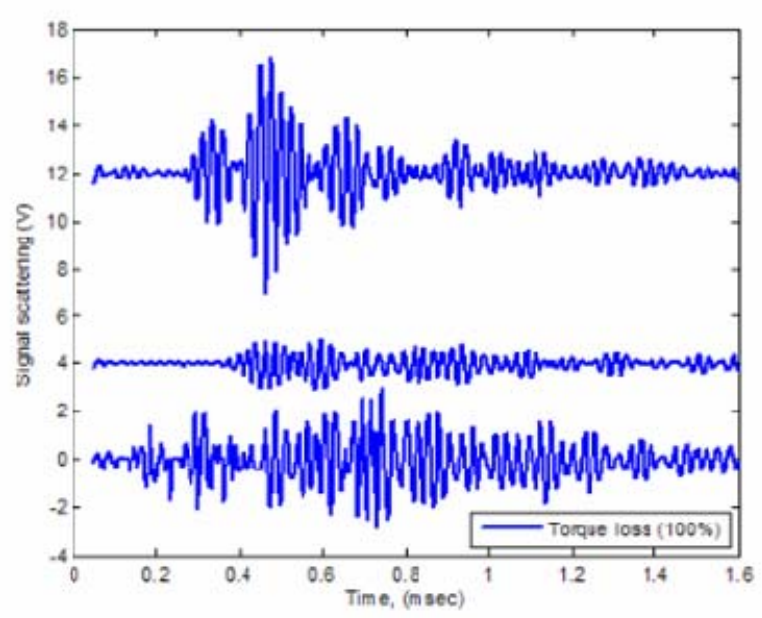

d) $100 \%$ torque loss

Figure 3-1-27. Scattering signals, B0 torque loss, E4 $40 \mathrm{kHz}$ excitation. 


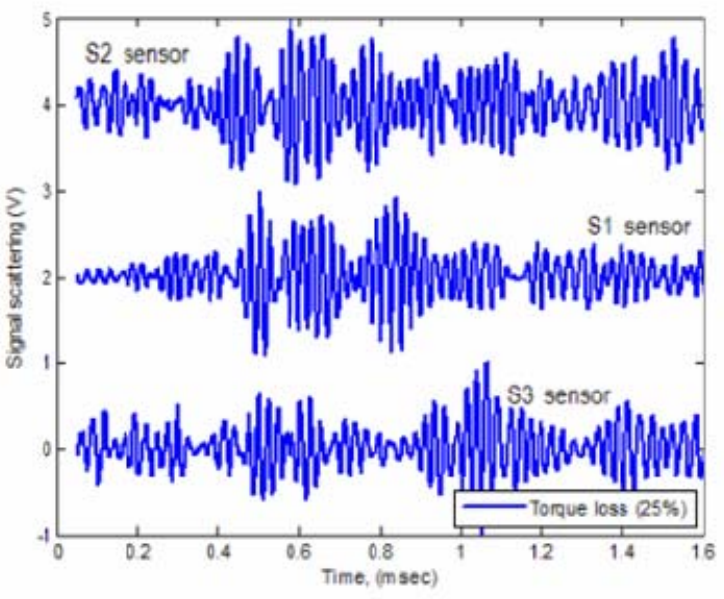

a) $25 \%$ torque loss

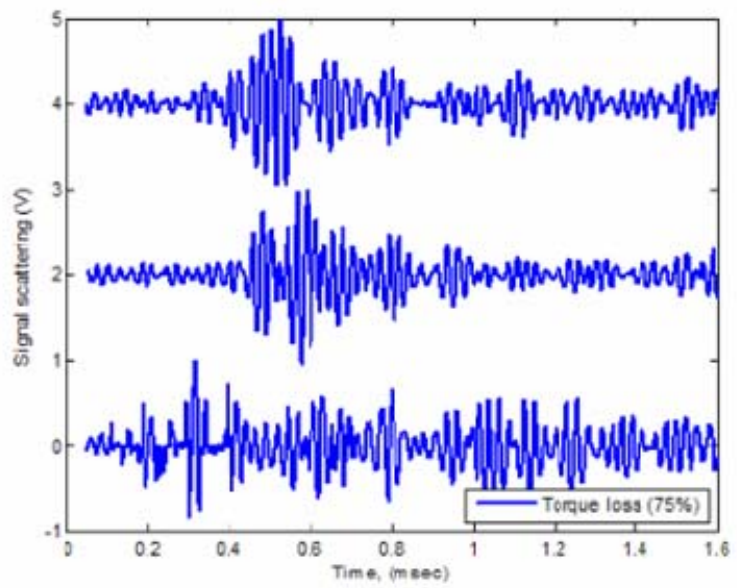

c) $75 \%$ torque loss

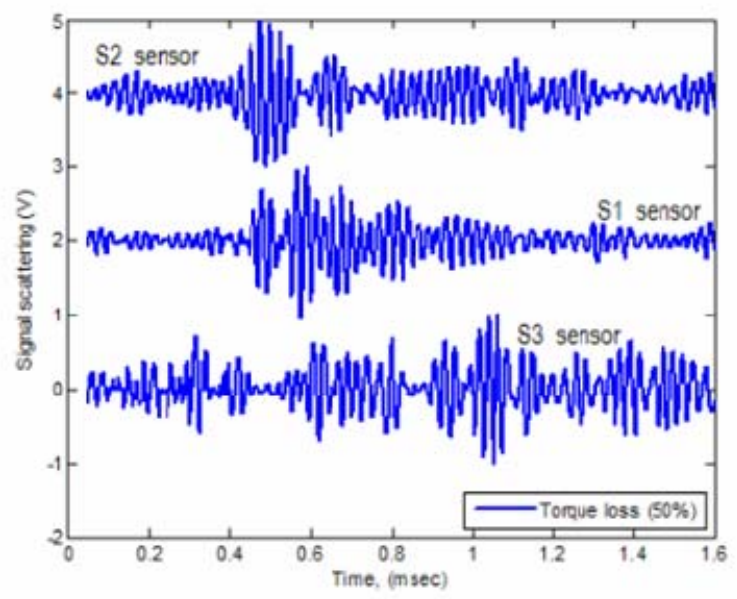

b) $50 \%$ torque loss

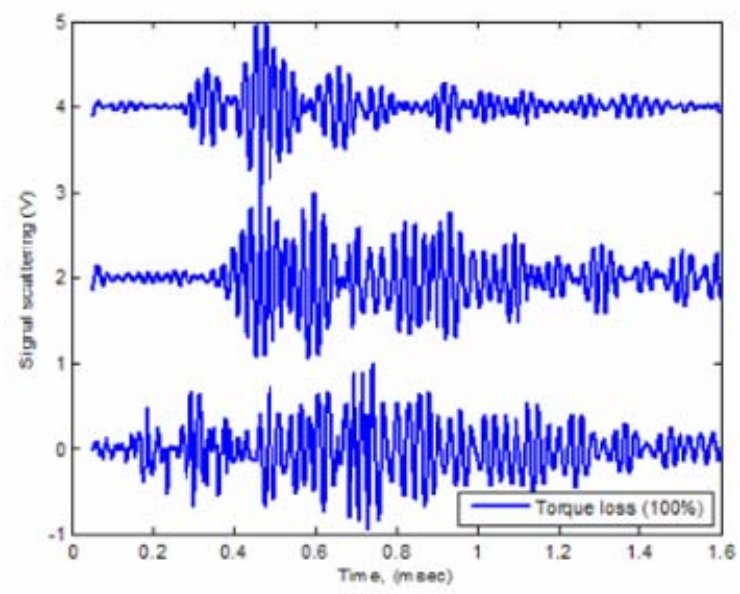

d) $100 \%$ torque loss

Figure 3-1-28. Normalized scattering signals, B0 torque loss, E4 $40 \mathrm{kHz}$ excitation.

Figure 3-1-29 and Figure 3-1-30 present the damage index using the energy magnitudes due to frequency response $\left(D I_{f}\right)$ and using the signals in time $\left(D I_{t}\right)$ at $\mathrm{S} 1, \mathrm{~S} 2$ and S3 under an E4 excitation case. Even when actuation is performed on the same composite panel (composite 2) where damage is present, the damage detection capabilities of each sensor using time and frequency response magnitudes are favorable under the E4 excitation configuration with respect to Bolt 0 torque loss. 


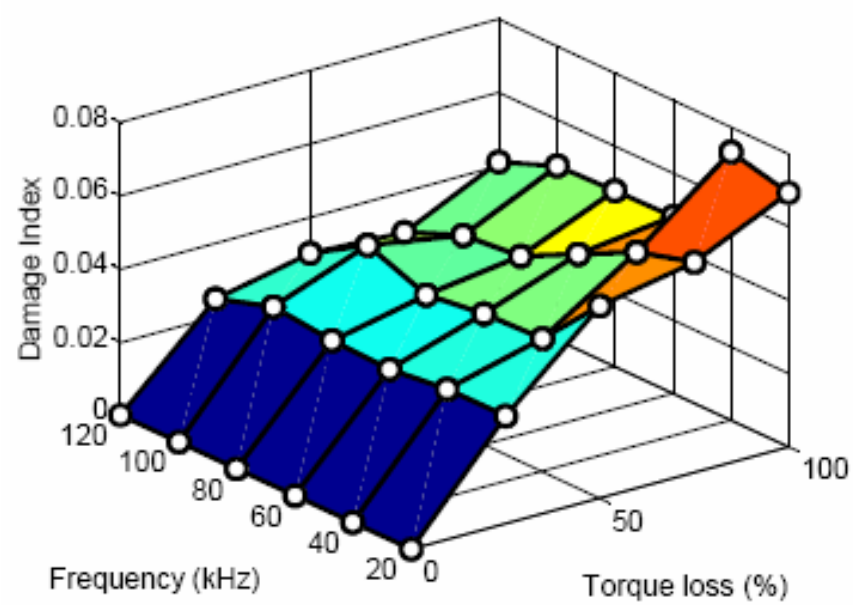

(a) $\mathrm{S} 1$ sensor

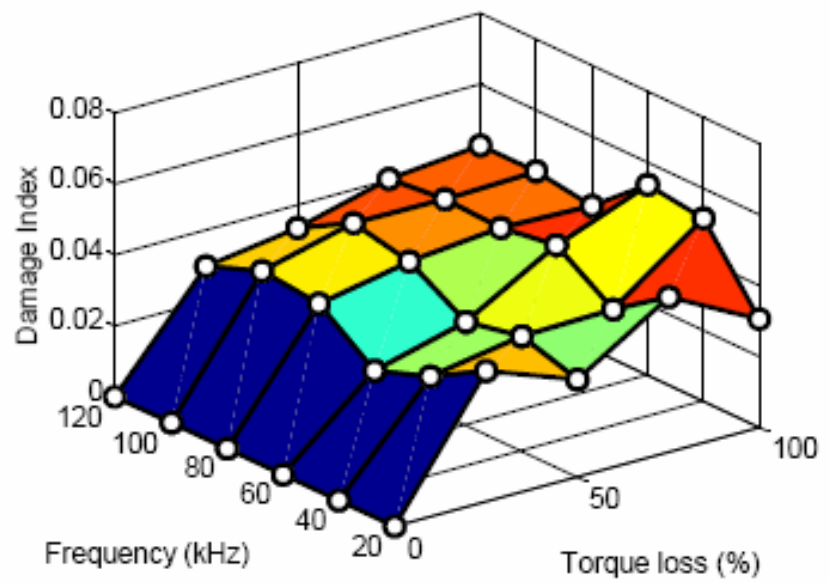

(b) S2 sensor

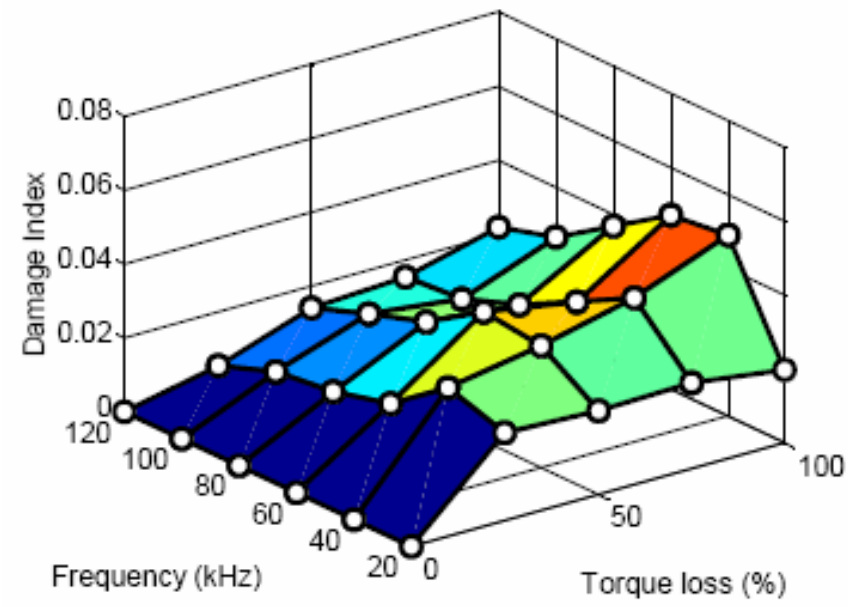

(c) S3 sensor

Figure 3-1-29. Damage index using the energy magnitudes in frequency vs. B0 torque loss and excitation frequency, E4 excitation. 


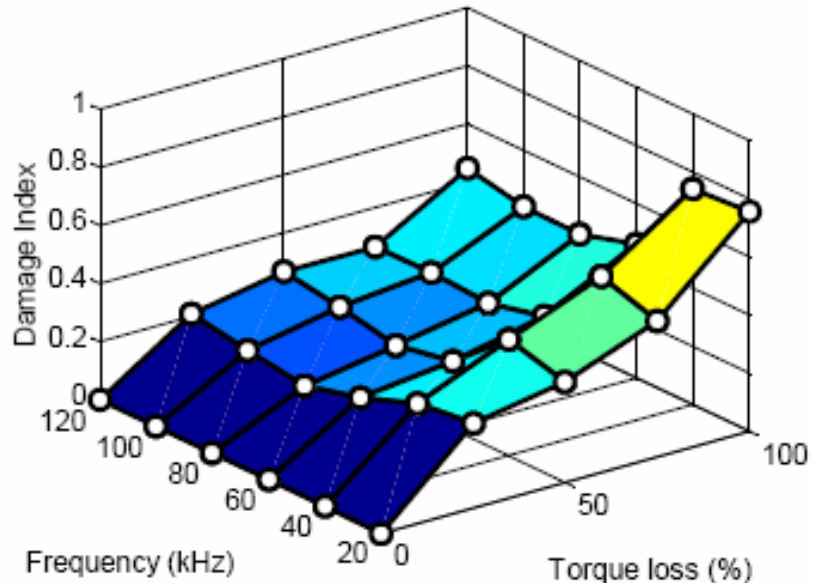

(a) $\mathrm{S} 1$ sensor

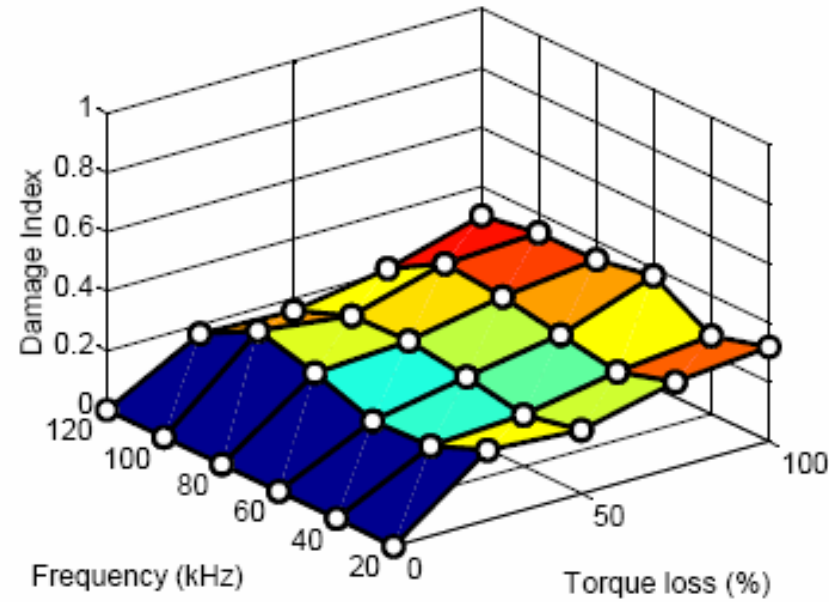

(b) S2 sensor

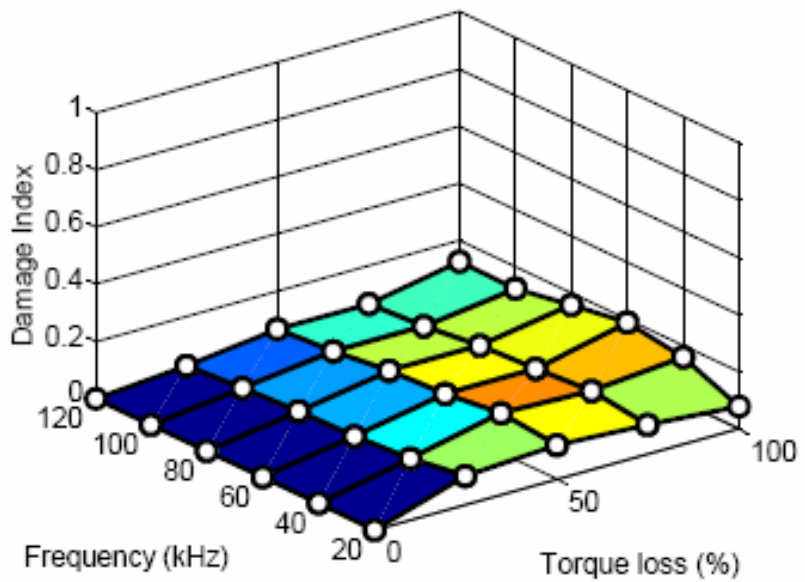

(c) S3 sensor

Figure 3-1-30. Damage index using the signals in time vs. B0 torque loss and excitation frequency, E4 excitation. 
Figure 3-1-30 displays smaller damage sensitivity in S3 as compared to the damage sensitivity in S1 and S2 sensors. This same trend was displayed in the S4 sensor under E3 excitation, once again reiterating the smaller capacity for damage detection within sensors that are located on the same composite panel as frequency actuation.

Figure 3-1-31 presents the normalized average damage index (DI average $)$ of the jointed composite panel due to torque loss in Bolt 0 for E4 excitation. As in the three previous excitation cases, the damage index trend-lines of all three sensors indicates an immediate increase in damage index from the undamaged scenario to the scenario of $25 \%$ torque loss, indicating the damage detection method's ability to detect small quantities of damage due to torque loss in the jointed composite specimen. The trend line due to S3 sensor is lower than the trend lines due to the S1 and S2 sensors. Similar to the S4 trend line in Figure 3-1-23, this indicates the S3 sensor's decreased ability to perceive damage within the composite joint as compared to the S1 and S2 sensors. 


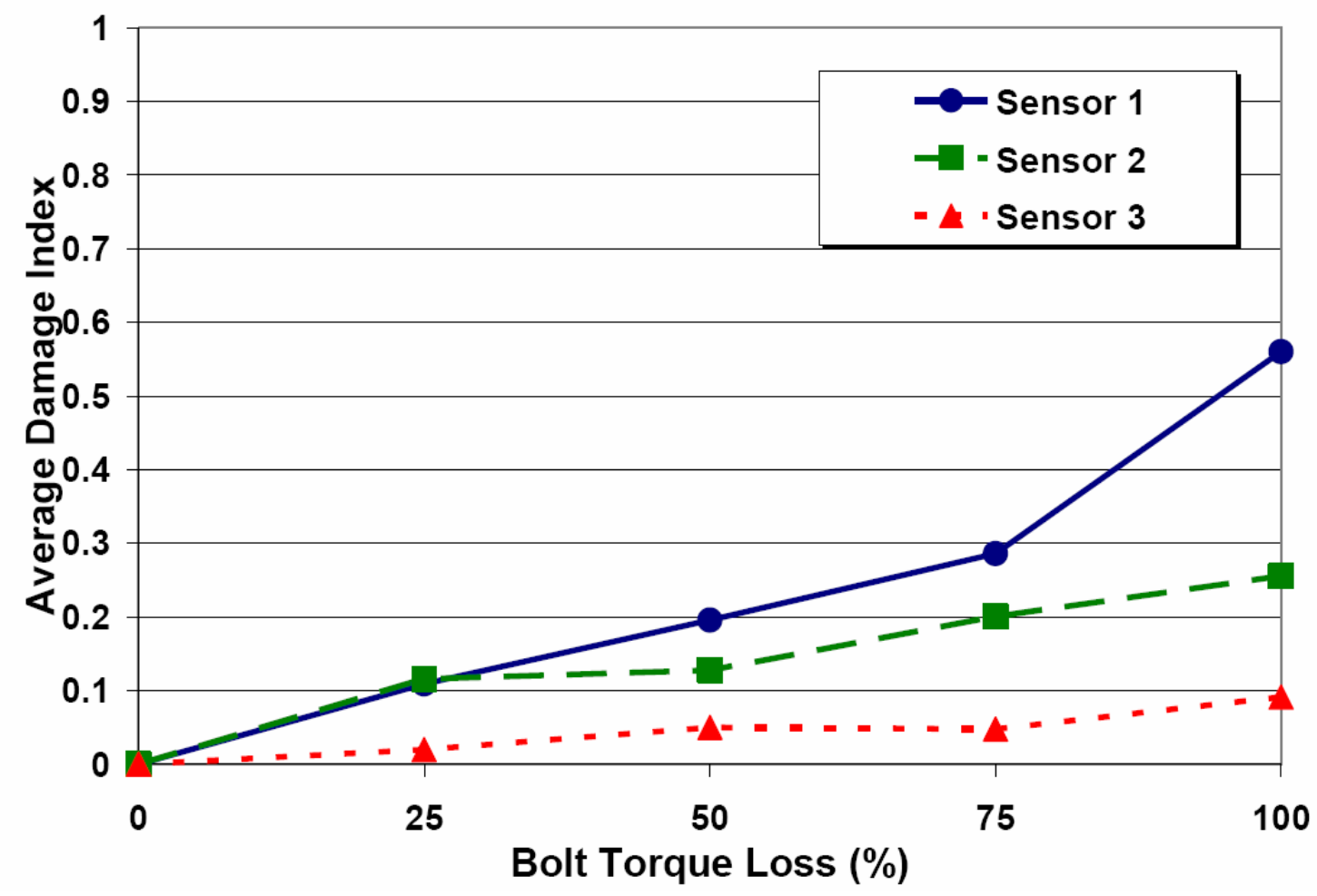

Figure 3-1-31. Average damage index, B0 torque loss, E4 excitation.

In Figure 3-1-31, the magnitude of average damage index from the $\mathrm{S} 1$ sensor is higher than the average damage index values associated with S2 at torque loss values above 25\%. This is similar to the trend in Figure 3-1-15, where the magnitude of average damage index from the S3 sensor was higher than the damage index from S4. Correspondingly, the trend presents the notion that under E4 excitation, the sensor in Quadrant 1 indicates a higher incidence of damage than the sensor in Quadrant 2 for torque loss values greater than $25 \%$.

Figure3-1-32 presents the total damage index under E4 excitation with respect to Bolt 0 torque loss. The total damage index contribution from the S1 and S2 sensors across the joint makes up nearly the entire damage index contribution from all three sensors, S1, S2 and S3. As noted in previous total damage index figures, this again 
displays the importance that when damage is present within the joint, sensing ability at locations across the joint contributes greatly toward damage detection capability.

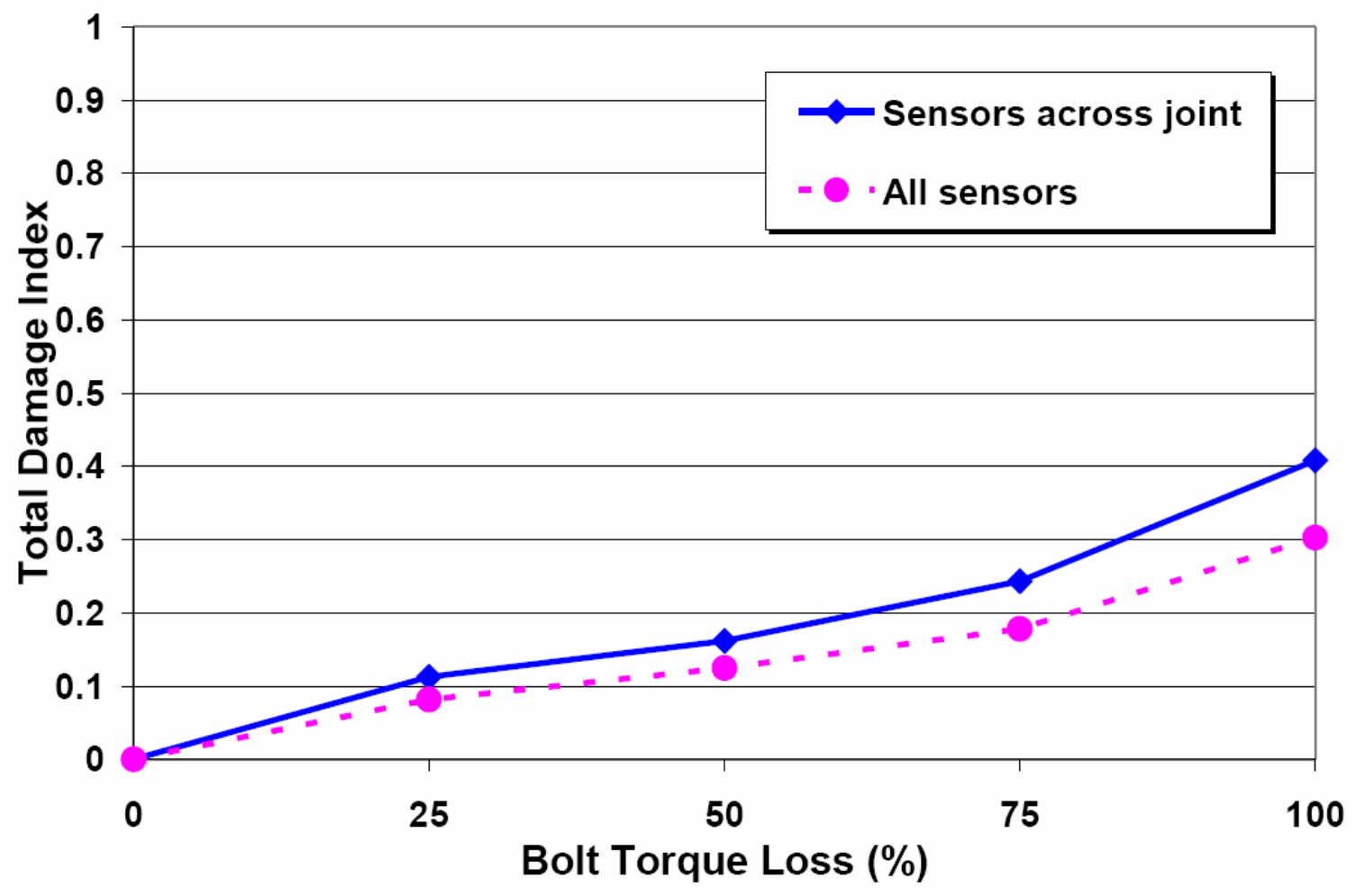

Figure 3-1-32. Total damage index, B0 torque loss, E4 excitation.

\subsubsection{Comparison of Bolt 0 excitation configurations}

Figure 3-1-33 shows the side-by-side comparison of the normalized average damage index of the composite jointed panel due to Bolt 0 torque loss for each excitation configuration case (E1, E2, E3 and E4). For each excitation configuration, the damage detection capability was favorable and provided insight into the presence and location of damage due to bolt torque loss. It is possible to use the average damage index for each excitation case to provide a rough estimate of the location of damage due to torque loss within the jointed composite panel by analyzing and comparing the overall trend-line 
from each sensor on each of the plots. As seen from Figure 3-1-33, the average damage index is as follows:

$$
\begin{array}{rlrl}
D I_{E 1, S 3} & \geq D I_{E 1, S 4}>>D I_{E 1, S 2} & & \text { Figure 3-1-33 (a) } \\
D I_{E 2, S 3} & >D I_{E 2, S 4}>>D I_{E 2, S 1} & & \text { Figure 3-1-33 (b) } \\
D_{E 3, S 1} \geq D I_{E 3, S 2}>>D I_{E 3, S 4} & \text { Figure 3-1-33 (c) } \\
D I_{E 4, S 1}>D I_{E 4, S 2}>>D I_{E 4, S 3} & \text { Figure 3-1-33 (d) }
\end{array}
$$

The analysis and comparison of damage index trends under each excitation case implies that the damage due to torque loss is in the common area in-between sensors S1 and S3, within Quadrant 1 or Quadrant 3. The average damage index values from S3 and S4 under E1 excitation are nearly indistinguishable. Similarly, the average damage index values from S1 and S2 under E3 excitation are nearly indistinguishable among the percentage torque loss values. It can be determined that, when actuation exists within the quadrants of possible damage, the average damage index trends can not provide great insight toward precise damage localization. 


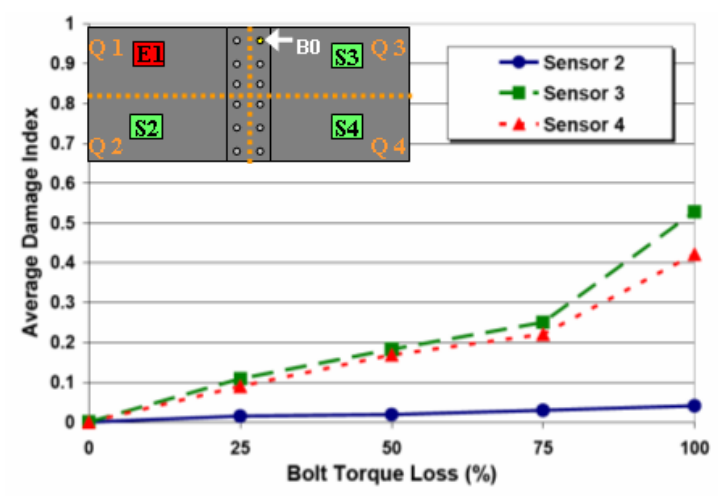

(a) E1 excitation

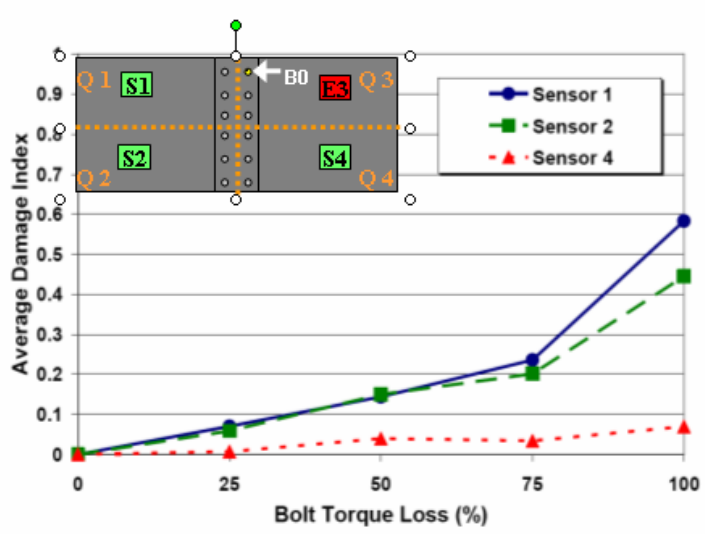

(c) E3 excitation

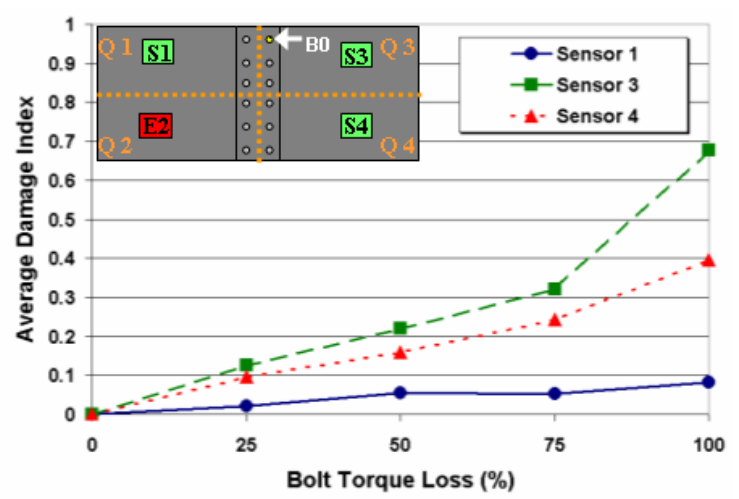

(b) E2 excitation

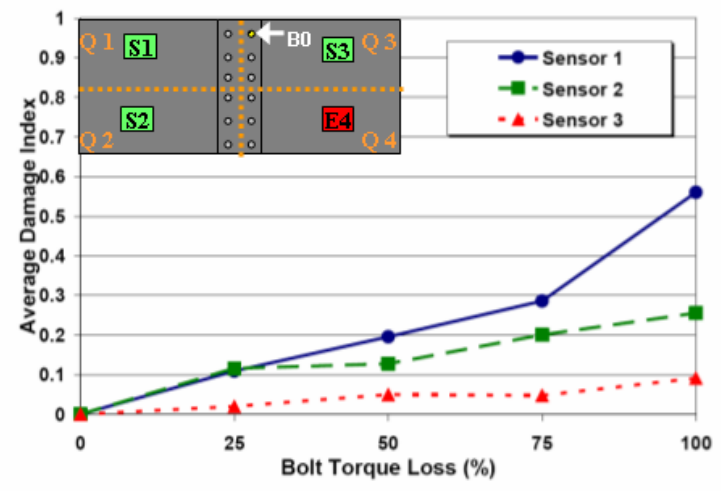

(d) E4 excitation

Figure 3-1-33. Comparison of normalized average damage index, B0 torque loss.

Focusing on only excitation cases under E2 and E4, there is a clear distinction between the average damage index trends; $D I_{E 2, S 3}$ is distinguishably larger than $D I_{E 2, S 4}$ and $D I_{E 4, S 1}$ is distinguishably larger than $D I_{E 4, S 2}$ over the torque loss values. When actuation is present in quadrants furthest from the predicted damage areas, the sensing information provides great insight toward the localization of damage within the composite jointed panel. Comparing the average damage index from Figure 3-1-33 (b) and Figure 3-1-33 (d) clearly shows:

$$
D I_{E 2, S 3}>D I_{E 4, S 1}
$$


The information is isolated to show this comparison graphically in Figure 3-1-34.

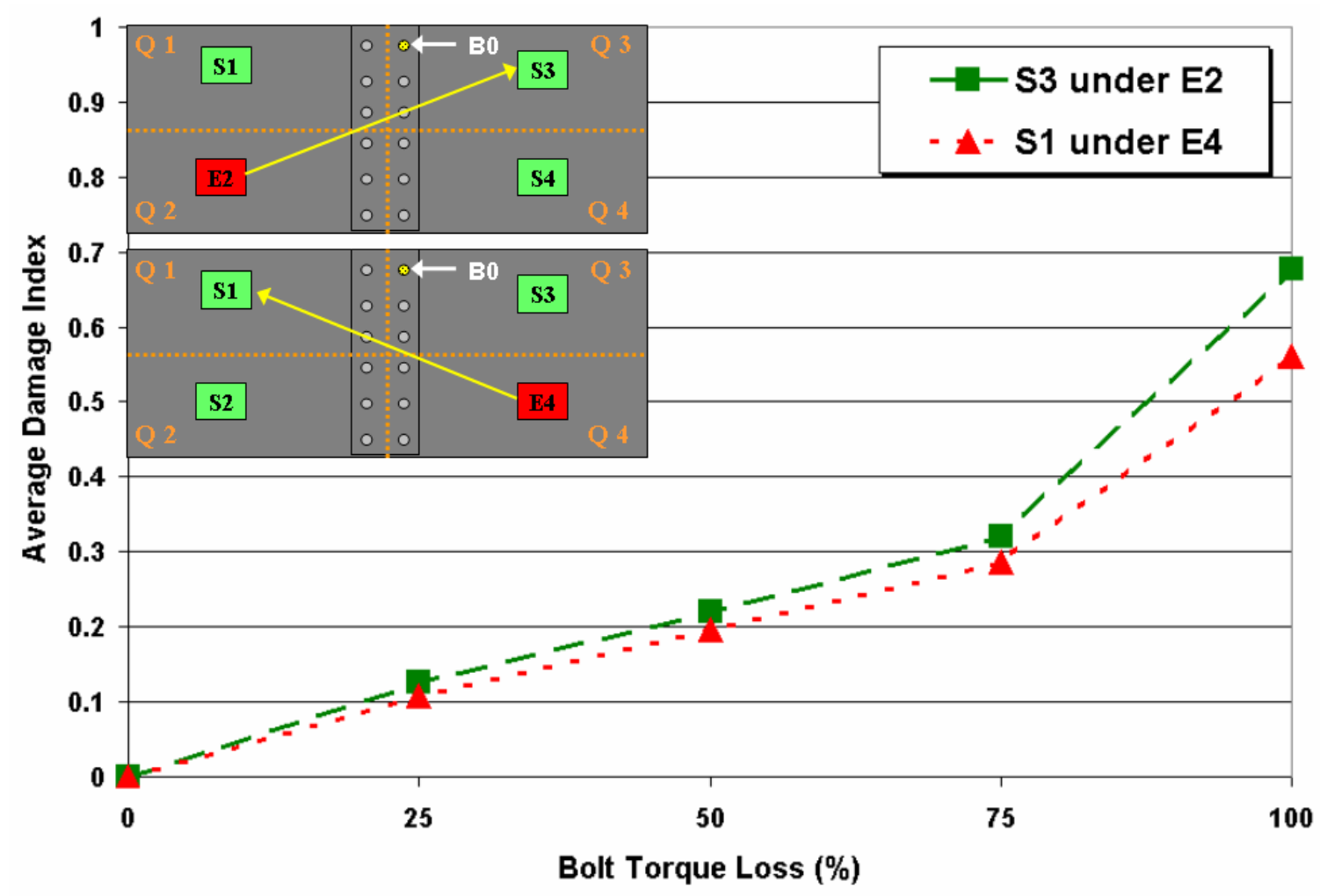

Figure 3-1-34. Comparison of normalized average damage indices, B0 torque loss; $D I_{E 2, S 3}$ and $D I_{E 4, S 1}$.

This trend indicates a higher damage index within proximity to S3, thus the greater likelihood of damage due to bolt torque loss in Quadrant 3 as compared to Quadrant 1. Figure 3-1-35 shows the shaded area of the highest likelihood of damage. 


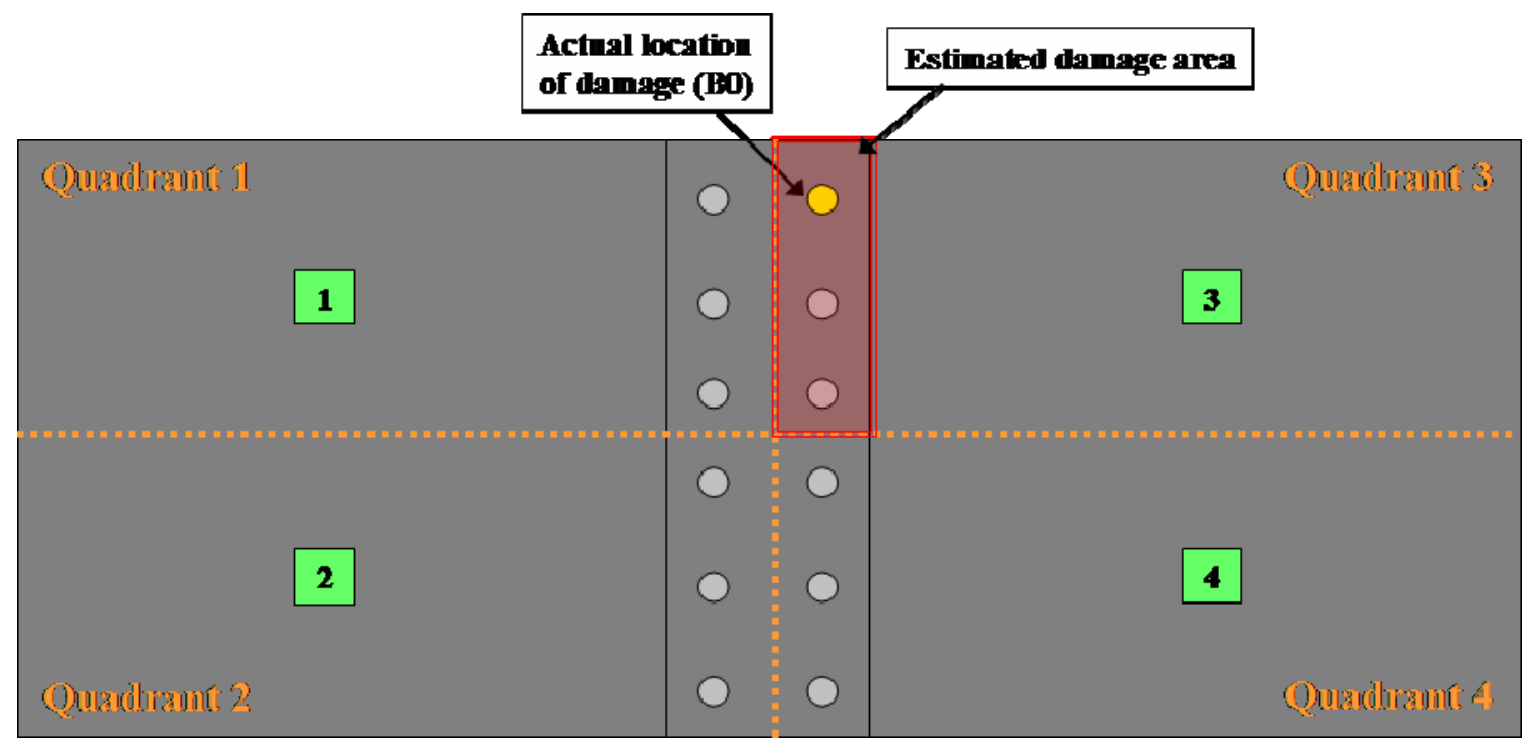

Figure 3-1-35. Estimated damage area due to B0 torque loss.

\subsubsection{Detection of smaller torque loss percentages}

In the previous sections, it was shown that the wireless smart sensor array system and the utilization of average damage index values as a method for data compression could accurately predict the incidence of bolt torque loss values as low as $25 \%(100 \%$ torque: $5 \mathrm{~N}-\mathrm{m}$ ). The examination of damage due to torque loss in Bolt 0 at smaller percentage values provided insight into the damage detection method's ability to sense smaller levels of damage. Under an E2 excitation configuration, Bolt 0 torque loss was varied by $5 \%, 10 \%, 15 \%$ and $20 \%$. These results were added to the previous E2 excitation information shown in section 3.1.2. Figure 3-1-36 shows the normalized average damage index of the composite jointed panel due to Bolt 0 torque loss. As shown, the damage detection method is capable of detecting the incidence of damage due to bolt torque loss for values as low as 5\%. Figure 3-1-37 shows the total damage index under E2 excitation with respect to the smaller Bolt 0 torque loss values. 
The bolt torque loss is adjusted using a hand torque wrench; using this method, there was difficulty adjusting the torque loss lower than $5 \%$. This value was used as the lowest threshold for damage detection using this method.

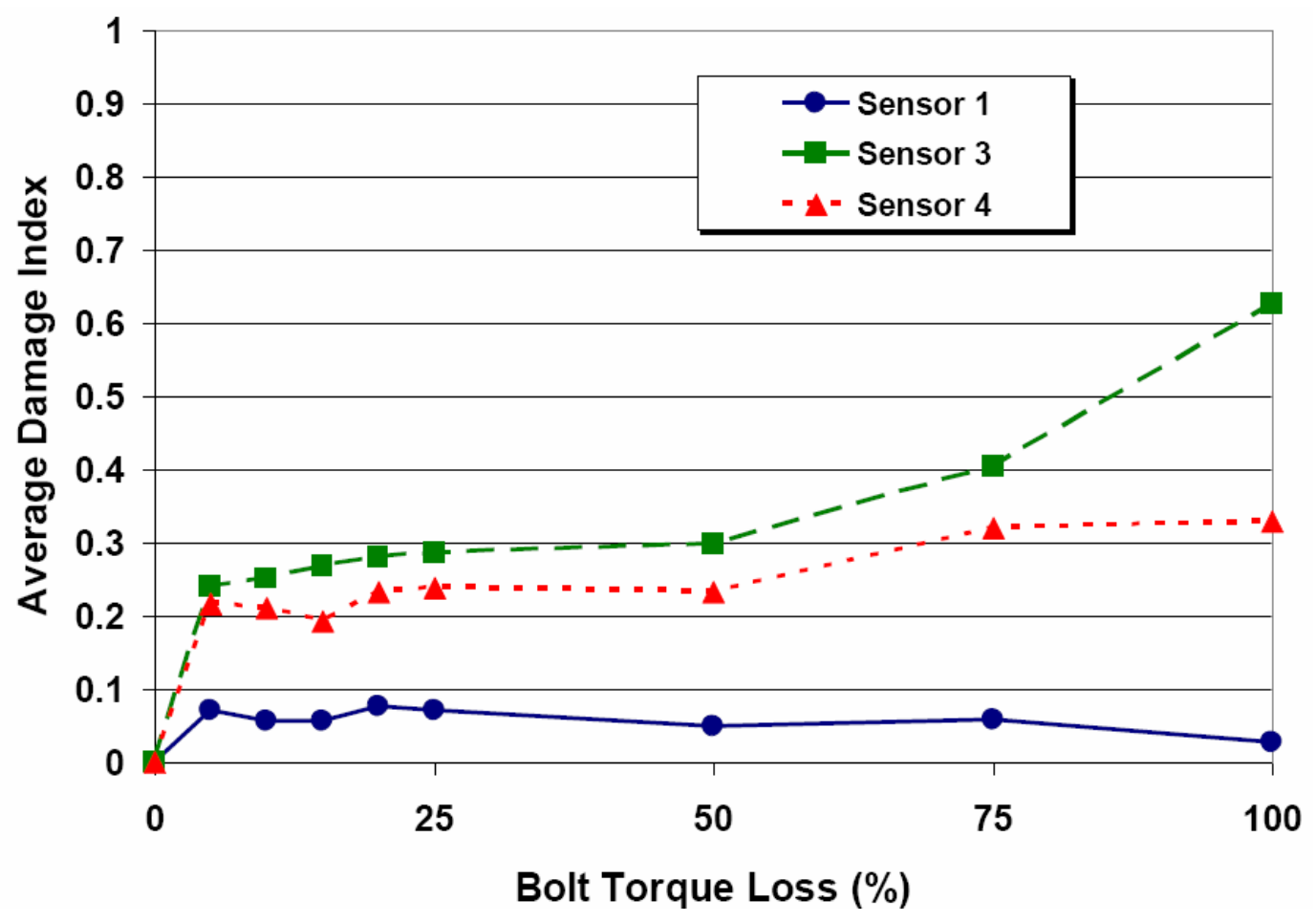

Figure 3-1-36. Normalized average damage index due to smaller B0 torque loss. 


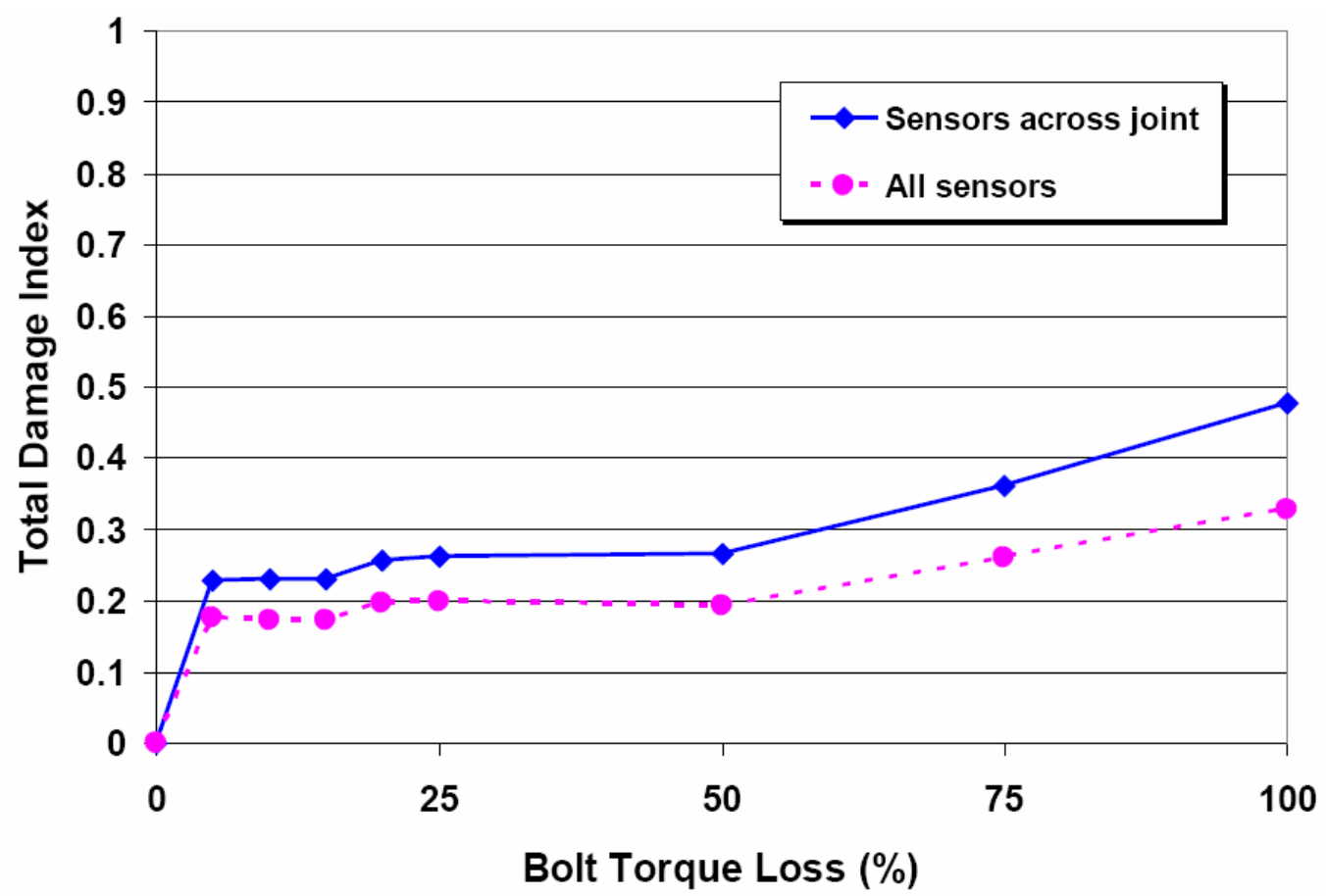

Figure 3-1-37. Total damage index due to smaller B0 torque loss.

\subsection{Bolt 1 damage due to torque loss}

In this section, the torque of Bolt 1 is manipulated in the same manner as Bolt 0 to evaluate the damage detection capabilities of the smart sensor array system on the jointed composite panel. Five torque values were tested at each of the four excitation configuration cases. Experimental results were compiled exactly as the Bolt 0 phase of experimentation. In this section, the results are presented in a condensed version to highlight the most important discoveries.

\subsubsection{Bolt 1, Excitation Case I}

Using the piezo-patch in Quadrant 1 (E1) for excitation of the jointed composite panel, sensing information is compiled at the three remaining sensors (S2, S3, S4) located within the remaining three quadrants. Figure 3-2-1 displays the excitation and sensing 
configuration and indicates the Bolt 1 location of simulated damage. Figure 3-2-2 represents the scattering signal for a $40 \mathrm{kHz}$ excitation frequency under E1 actuation. The signal scattering at S3 and S4 sensors increase in magnitude as torque loss increases. The scattering signals at sensors across the joint from the source of actuation (S3, S4) are smaller in magnitude than the signal from the sensor that shares a composite panel with the source of actuation (S2). This phenomenon is due to the incident signal loss as it passes through the composite joint.

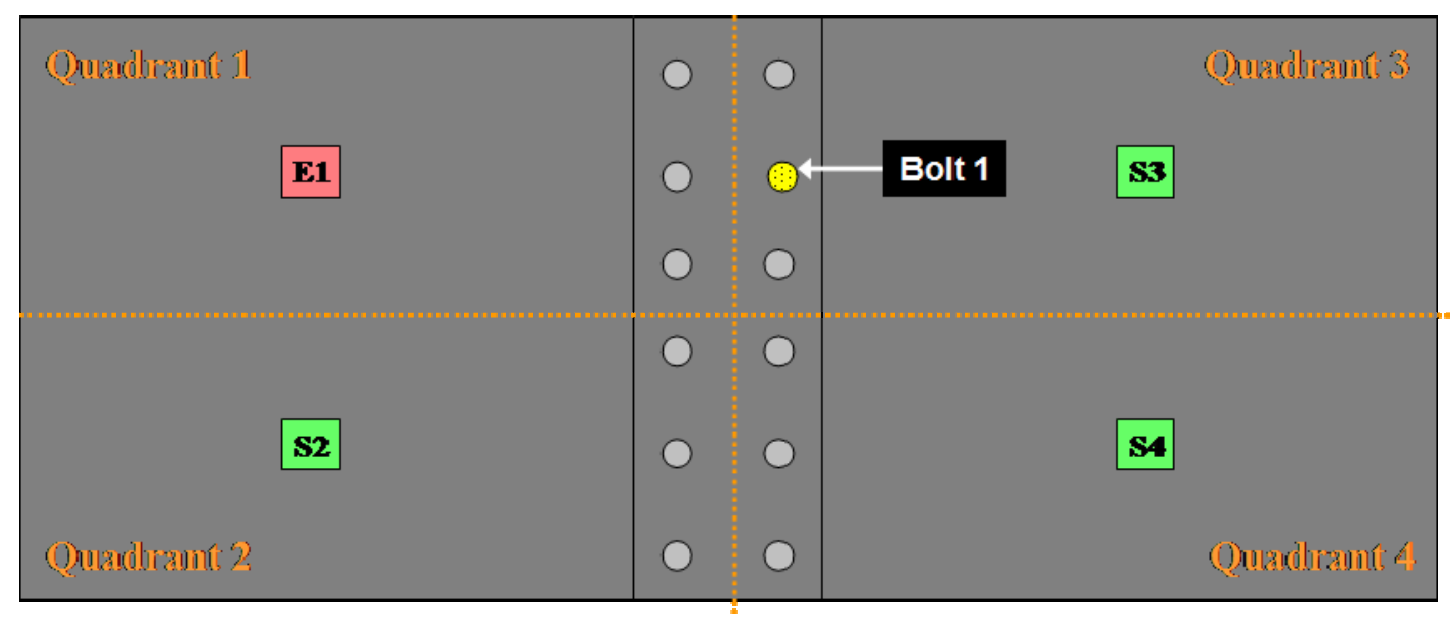

Figure 3-2-1. Excitation Case I, variable Bolt 1 torque loss. 


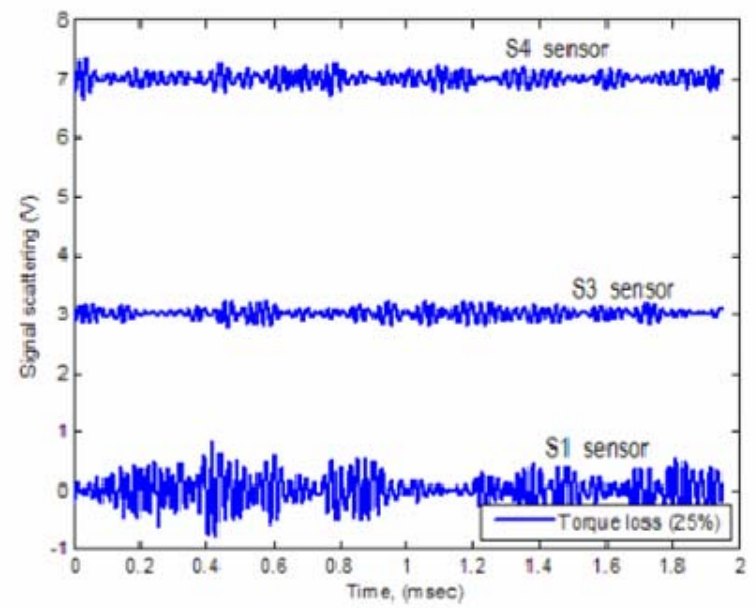

a) $25 \%$ torque loss

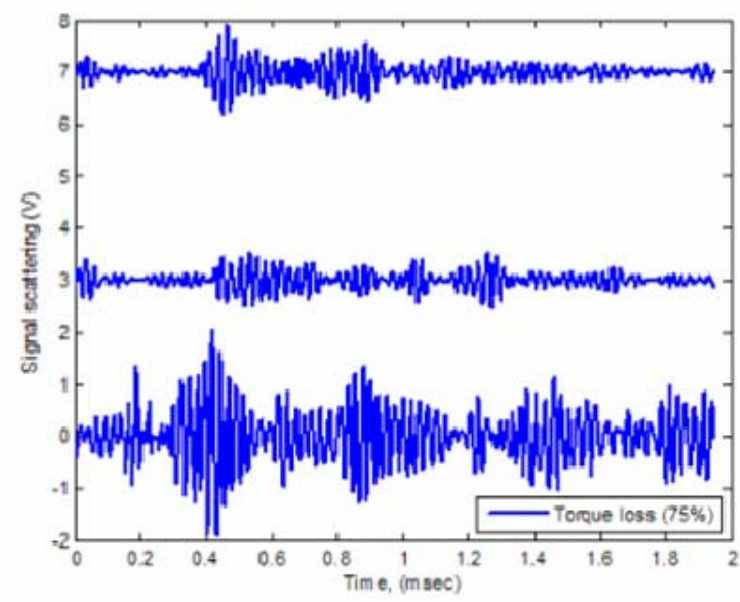

c) $75 \%$ tonque loss

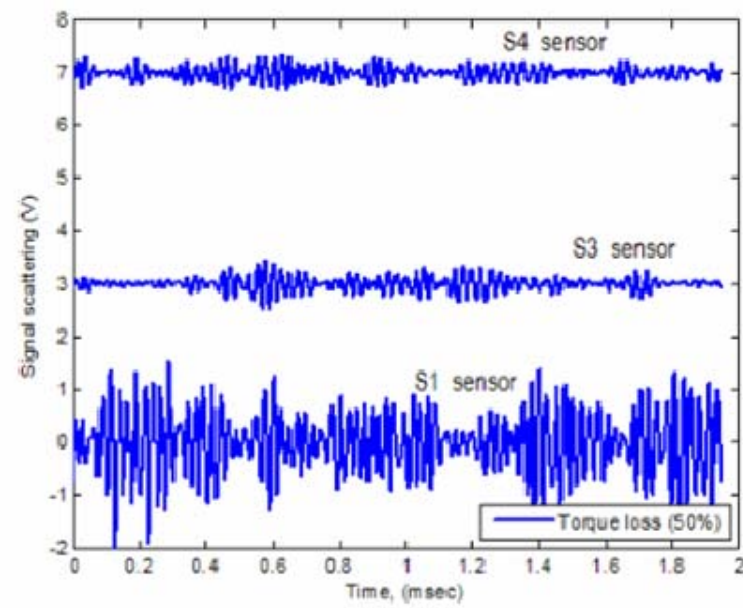

b) $50 \%$ torque loss

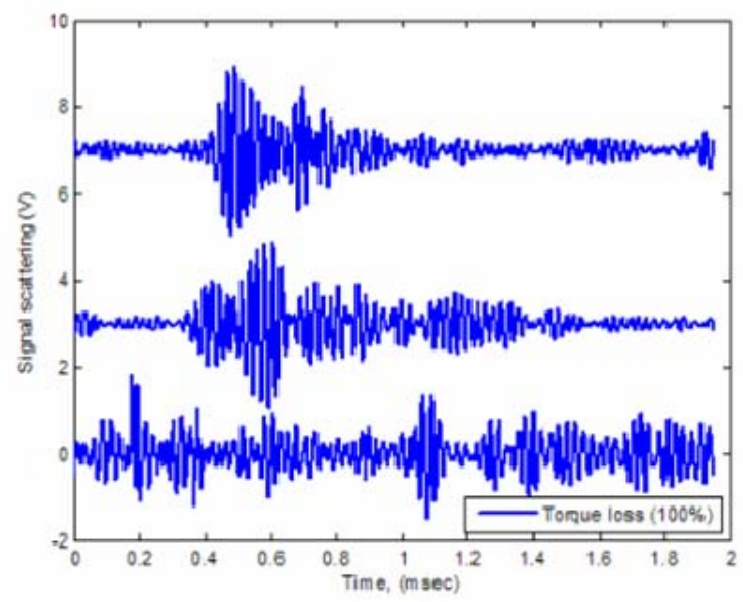

d) $100 \%$ torque loss

Figure 3-2-2. Scattering signals, B1 torque loss, E1 $40 \mathrm{kHz}$ excitation.

Figure 3-2-3 presents the damage index using the energy magnitudes due to frequency response $\left(D I_{f}\right)$ at S2, S3 and S4 under E1 excitation. Figure 3-2-4 presents the damage index using the signals in time $\left(D I_{t}\right)$ at $\mathrm{S} 2, \mathrm{~S} 3$ and $\mathrm{S} 4$ sensing locations. For each, the damage index variation increased with bolt torque loss. There was less variation in damage index over the range of excitation frequencies. The damage index is more sensitive to changes in damage level as compared to changes in excitation frequency. 


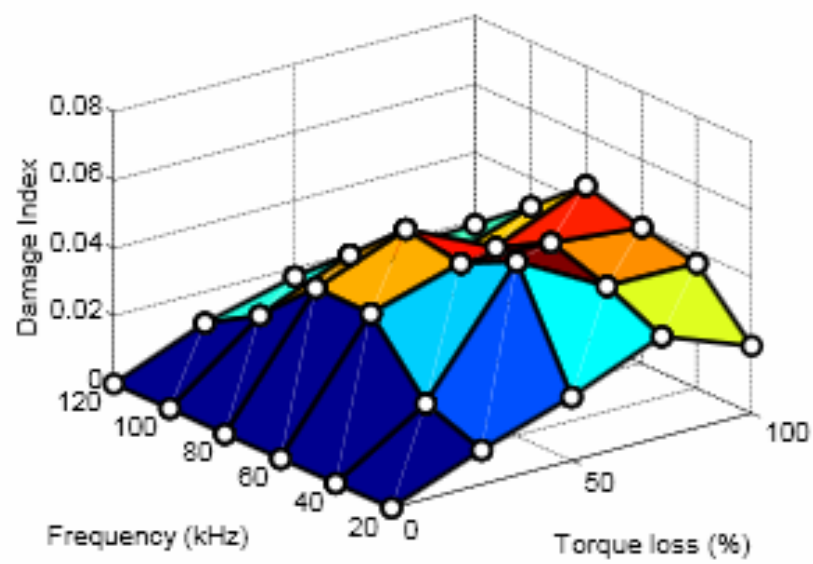

(a) S1 sensor

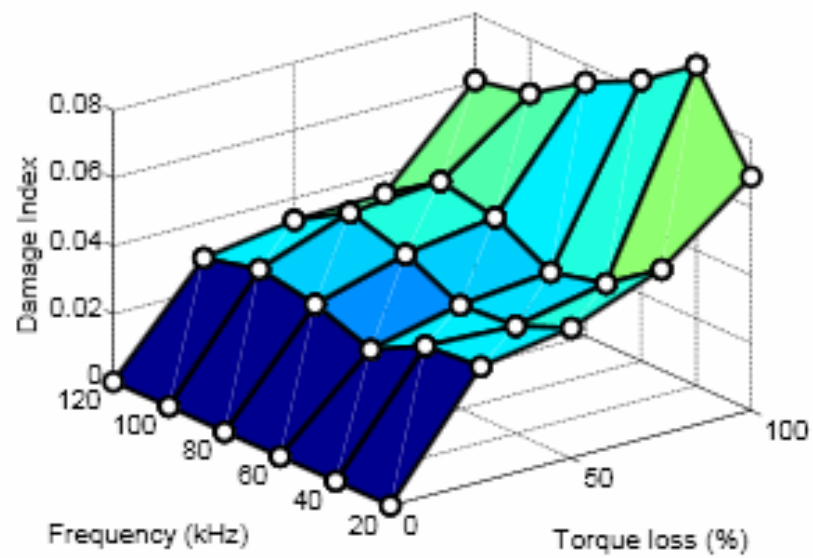

(b) $\mathrm{S} 3$ sensor

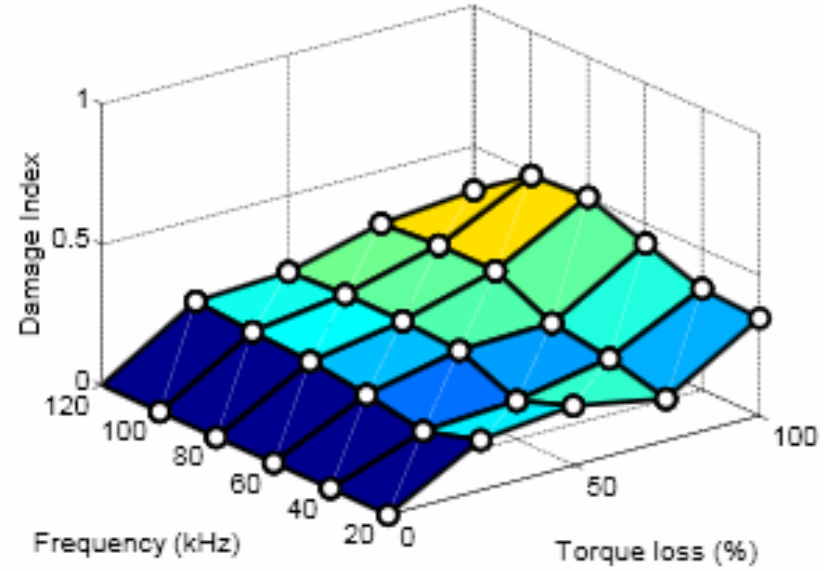

(c) $\mathrm{S} 4$ sensor

Figure 3-2-3. Damage index using the energy magnitudes in frequency vs. B1 torque loss and excitation frequency, E1 excitation. 


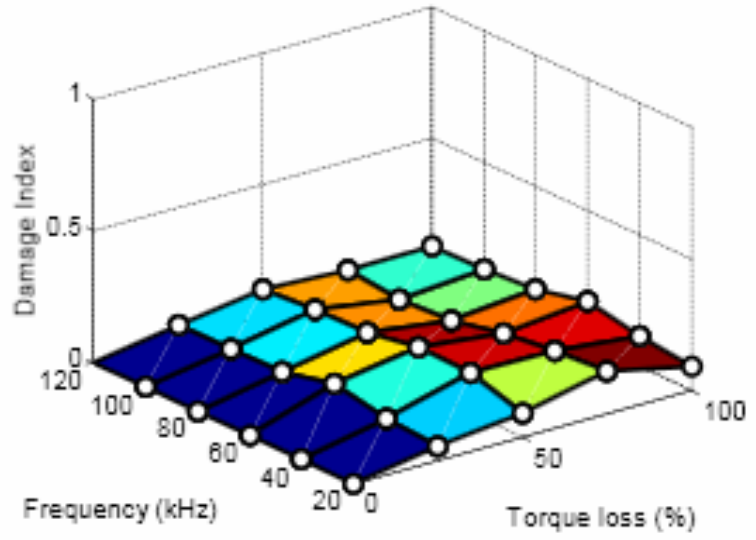

(a) $\mathrm{S} 1$ sensor

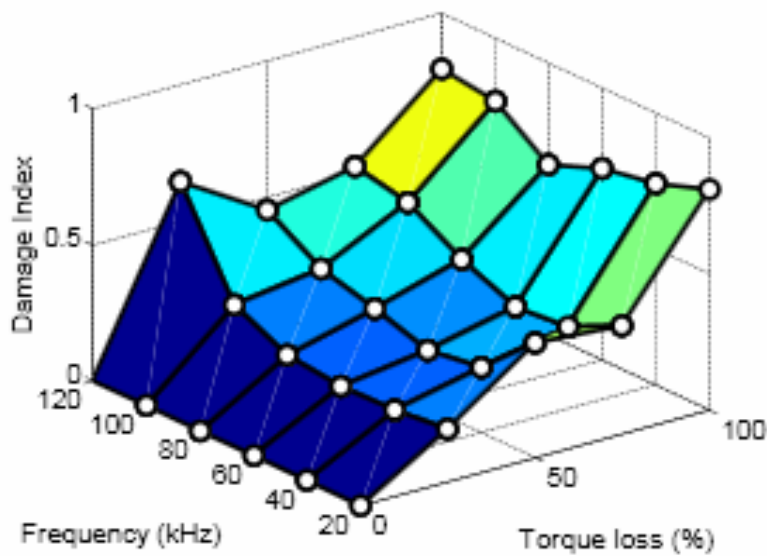

(b) $\mathrm{S} 3$ sensor

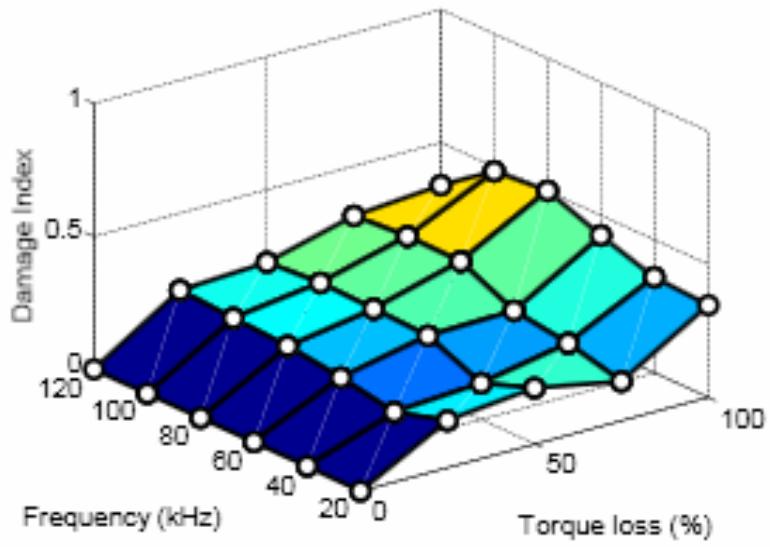

(c) S4 sensor

Figure 3-2-4. Damage index using the signals in time vs. B1 torque loss and excitation frequency, E1 excitation. 


\subsubsection{Bolt 1, Excitation Case II}

Using the piezo-patch in Quadrant 2 (E2) for excitation of the jointed composite panel, sensing information is compiled at the three remaining sensors (S1, S3, S4) located within the remaining three quadrants. Figure 3-2-5 displays the excitation and sensing configuration and indicates the Bolt 1 location of simulated damage. Figure 3-2-6 represents the scattering signal for a $40 \mathrm{kHz}$ excitation frequency under E2 actuation. The signal scattering at S3 and S4 sensors increase in magnitude as torque loss increases. The scattering signals at sensors across the joint from the source of actuation $(\mathrm{S} 3, \mathrm{~S} 4)$ are smaller in magnitude than the signal from the sensor that shares a composite panel with the source of actuation (S1). This phenomenon is due to the incident signal loss as it passes through the composite joint and is noted in previous chapter sections.

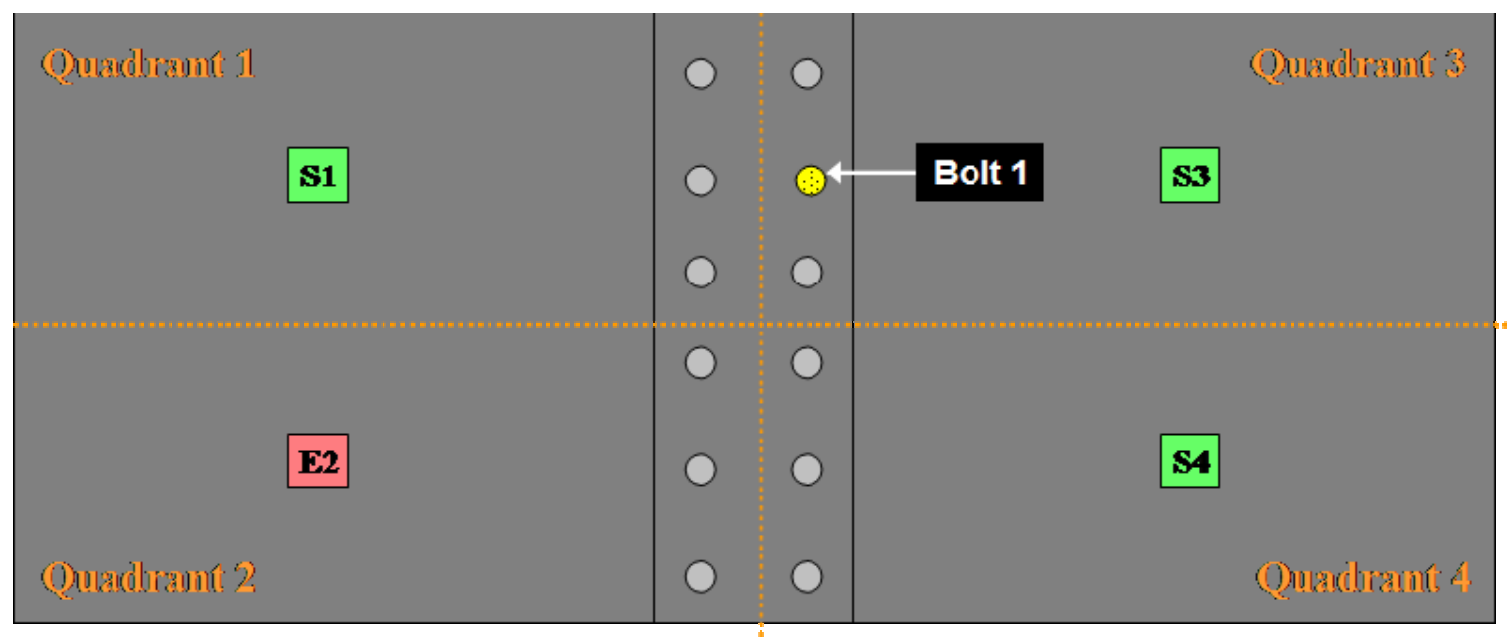

Figure 3-2-5. Excitation Case II, variable Bolt 1 torque loss. 


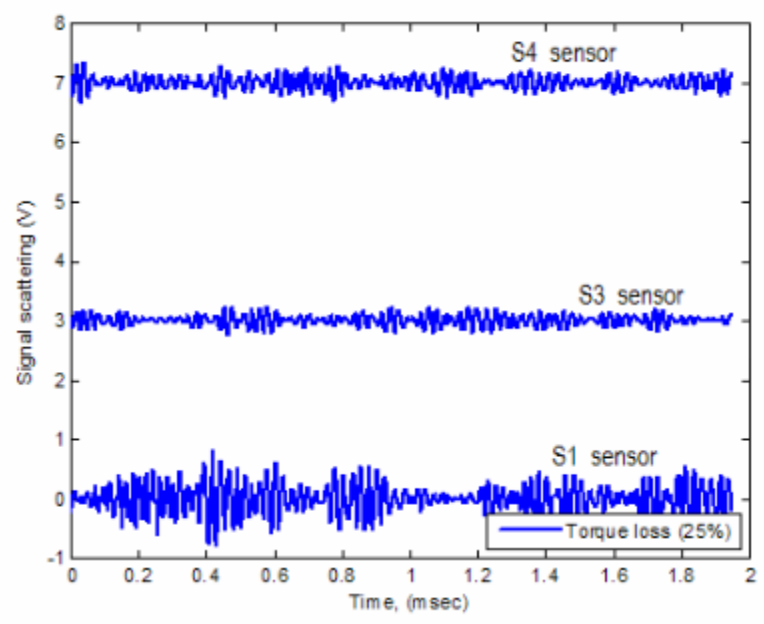

a) $25 \%$ torque loss

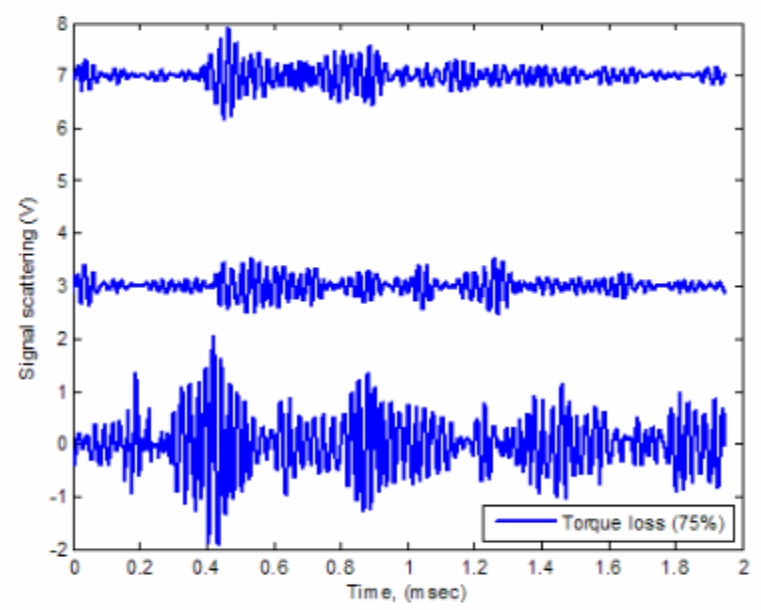

a) $75 \%$ torque loss

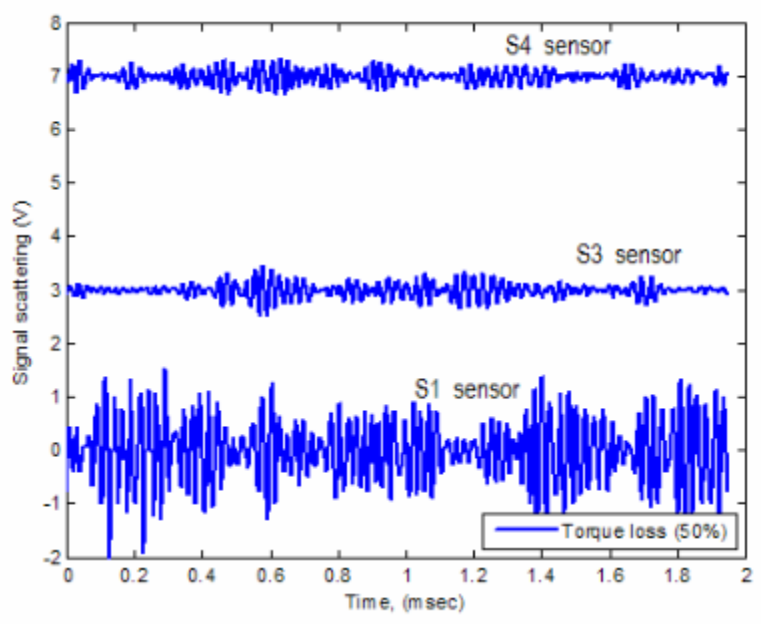

b) $50 \%$ torque loss

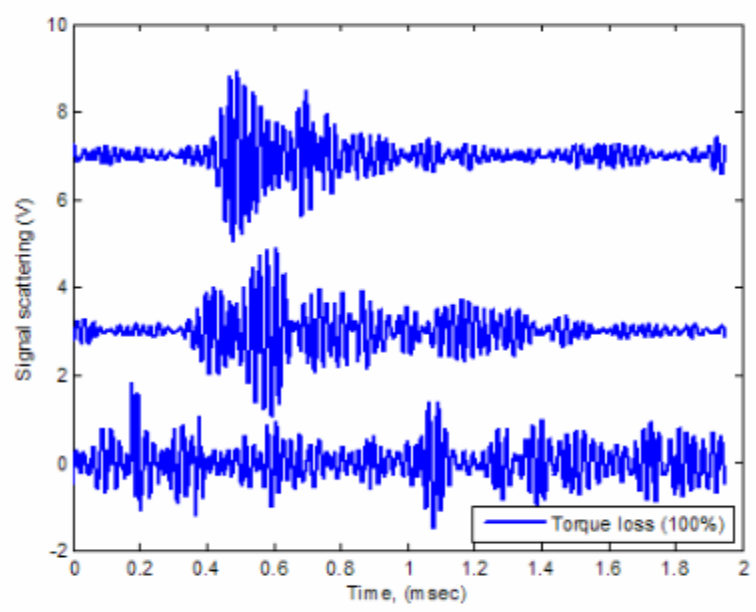

b) $100 \%$ torque loss

Figure 3-2-6. Scattering signals, B1 torque loss, E2 $40 \mathrm{kHz}$ excitation.

Figure 3-2-7 and Figure 3-2-8 present the damage index using the energy magnitudes due to frequency response $\left(D I_{f}\right)$ and using the signals in time $\left(D I_{t}\right)$ at $\mathrm{S} 1, \mathrm{~S} 3$ and S4 under E2 excitation. In each plot, the damage index is more sensitive to changes in damage level as compared to changes in excitation frequency. The damage detection capabilities of each sensor using time and frequency response magnitudes are favorable within the jointed composite panel due to Bolt 1 torque loss under the E2 excitation configuration. 


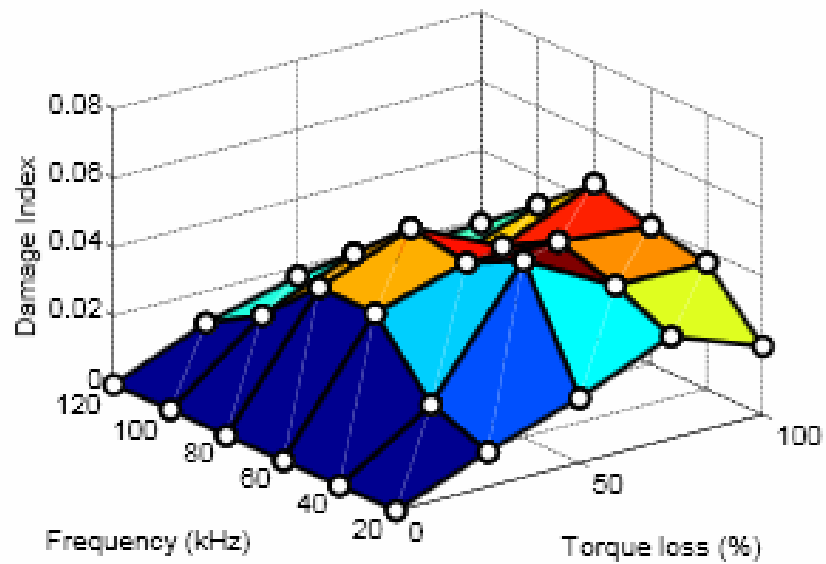

(a) $\mathrm{S} 1$ sensor

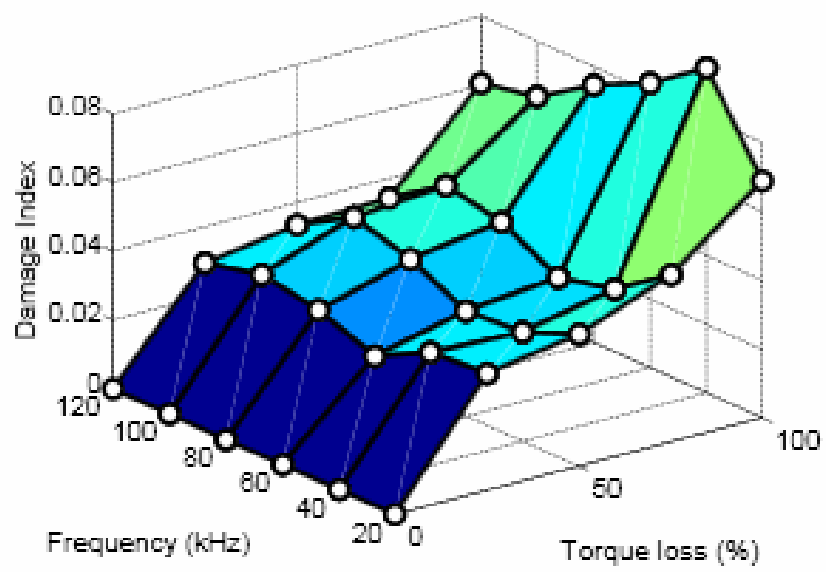

(b) $\mathrm{S} 3$ sensor

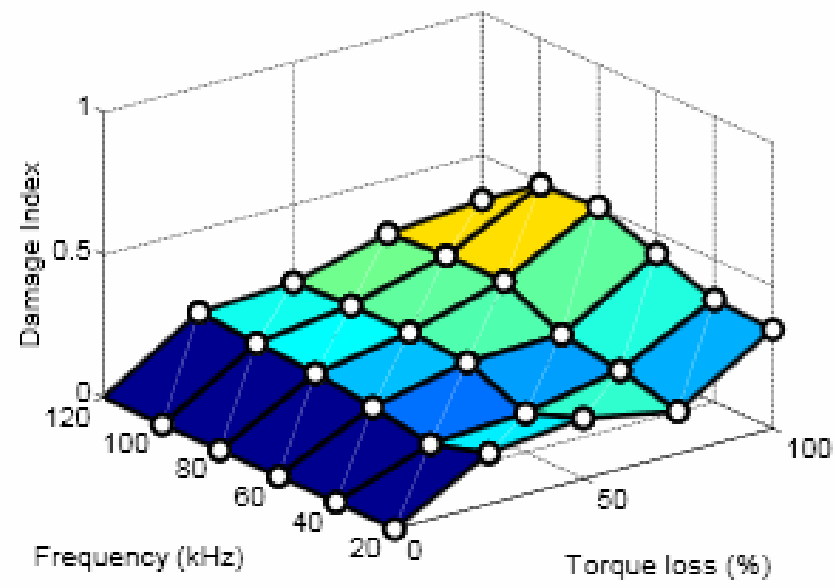

(c) $\mathrm{S} 4$ sensor

Figure 3-2-7. Damage index using the energy magnitudes in frequency vs. B1 torque loss and excitation frequency, E2 excitation. 


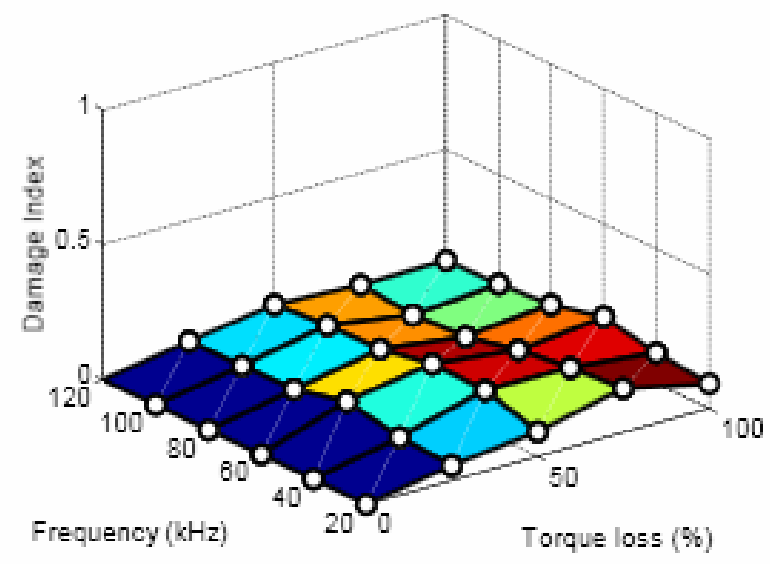

(a) $\mathrm{S} 1$ sensor

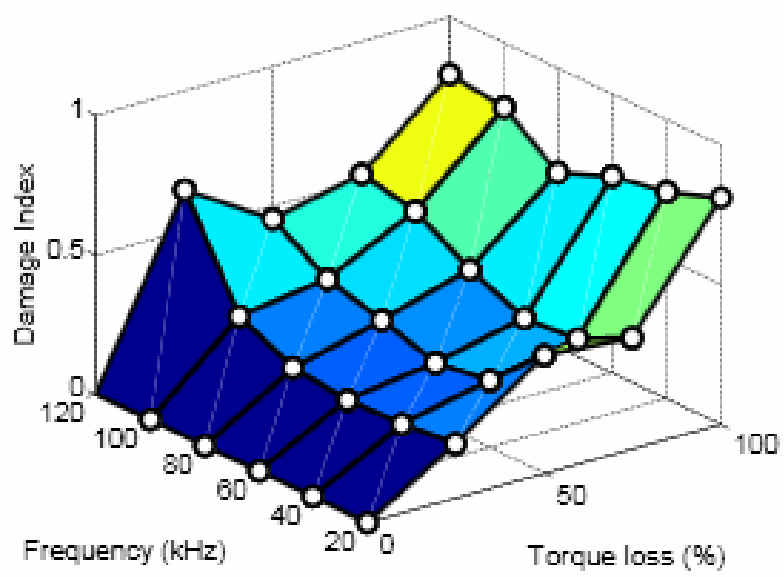

(b) $\mathrm{S} 3$ sensor

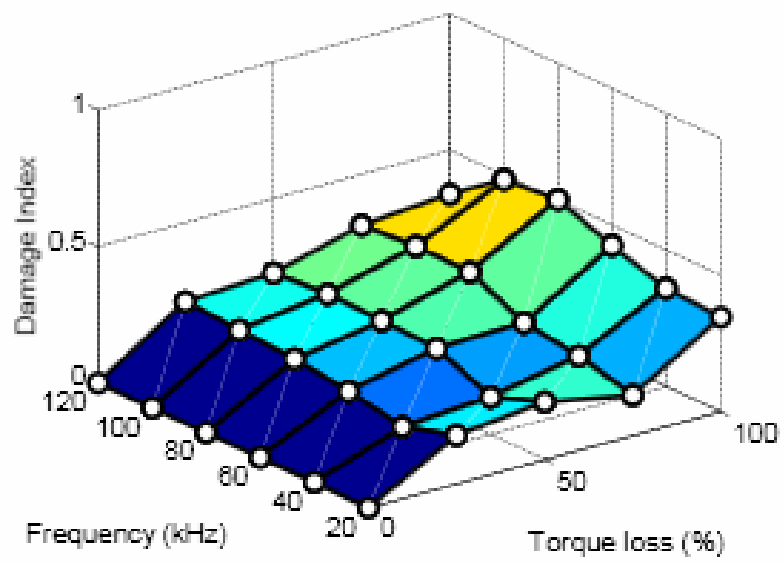

(c) S4 sensor

Figure 3-2-8. Damage index using the signals in time vs. B1 torque loss and excitation frequency, E2 excitation. 


\subsubsection{Bolt 1, Excitation Case III}

Using the piezo-patch in Quadrant 3 (E3) for excitation of the jointed composite panel, sensing information is compiled at the three remaining sensors (S1, S2, S4) located within the remaining three quadrants. In this excitation case, the actuation occurs in the same quadrant as the present damage due to torque loss. Figure 3-2-9 displays the excitation and sensing configuration and indicates the Bolt 1 location of simulated damage. Figure 3-2-10 represents the scattering signal for a $40 \mathrm{kHz}$ excitation frequency under E3 actuation. The signal scattering at S1 and S2 sensors increase in magnitude as torque loss increases. The scattering signals at sensors across the joint from the source of actuation $(\mathrm{S} 1, \mathrm{~S} 2)$ are smaller in magnitude than the signal from the sensor that shares a composite panel with the source of actuation (S4).

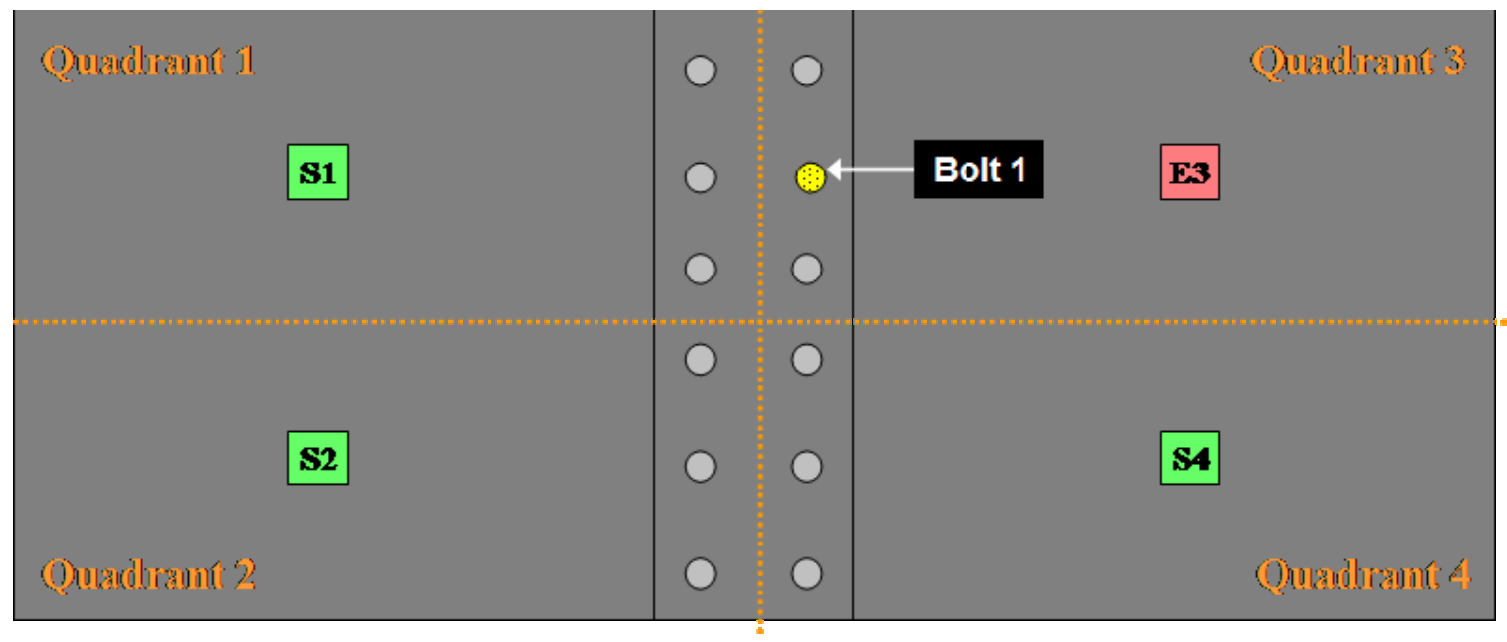

Figure 3-2-9. Excitation Case III, variable Bolt 1 torque loss. 


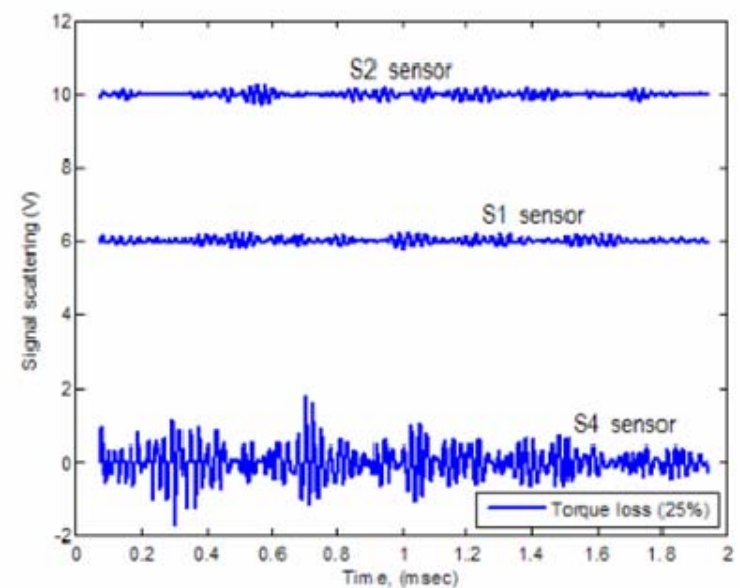

a) $25 \%$ torque loss

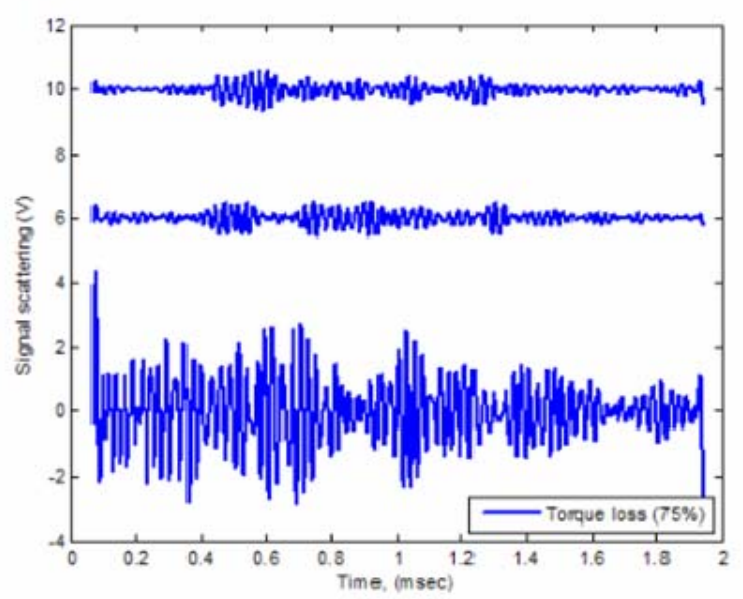

c) $75 \%$ torque loss

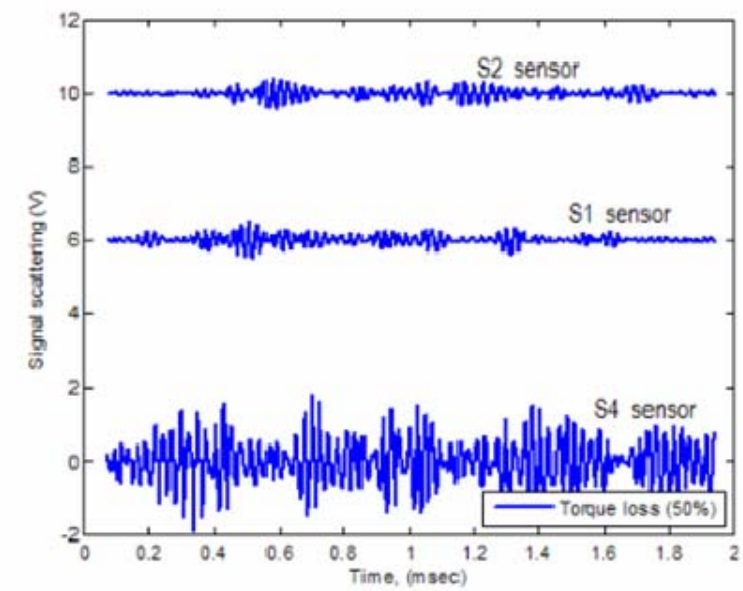

b) $50 \%$ torque loss

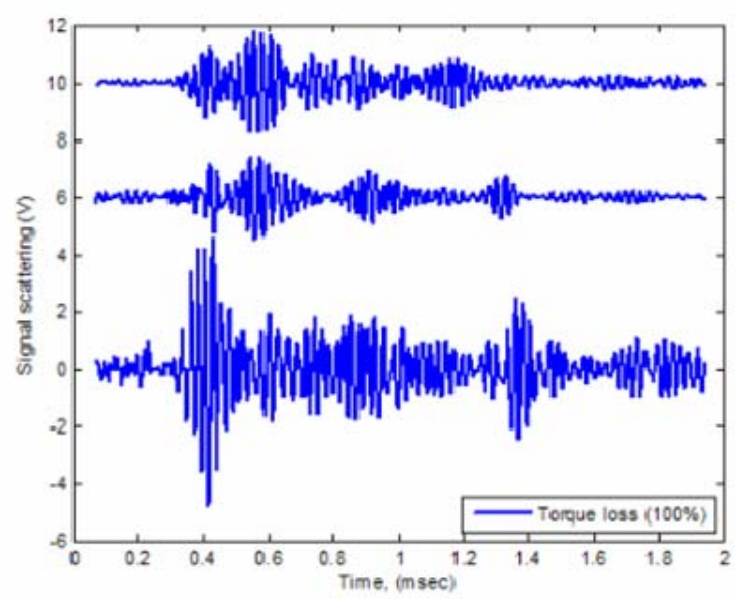

d) $100 \%$ torque loss

Figure 3-2-10. Scattering signals, B1 torque loss, E3 $40 \mathrm{kHz}$ excitation.

Figure 3-2-11 and Figure 3-2-12 present the damage index using the energy magnitudes due to frequency response $\left(D I_{f}\right)$ and using the signals in time $\left(D I_{t}\right)$ at $\mathrm{S} 1, \mathrm{~S} 2$ and S4 under E3 excitation. In each plot, the damage index is more sensitive to changes in damage level as compared to changes in excitation frequency. Despite the excitation source's close proximity to the actual damage location, the damage detection capabilities of each sensor using time and frequency response magnitudes are favorable within the jointed composite panel due to Bolt 1 torque loss under the E3 excitation configuration. 


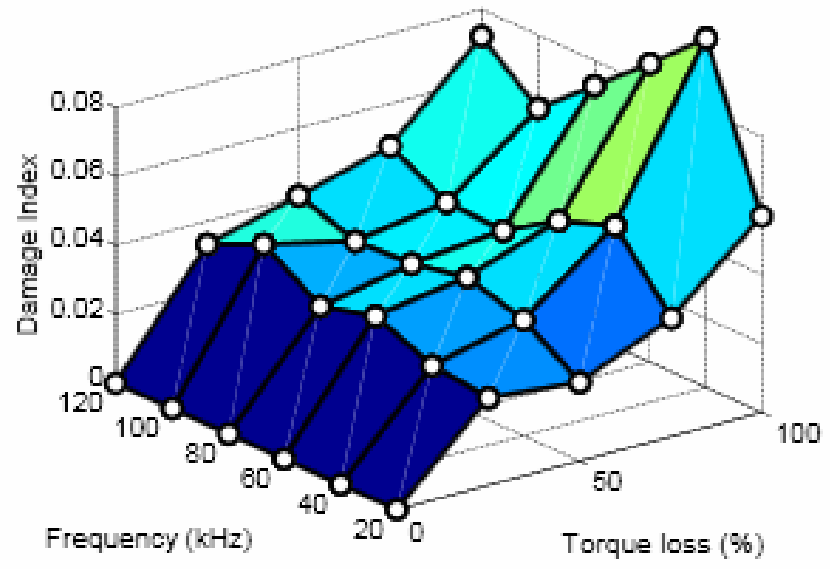

(a) S1 sensor

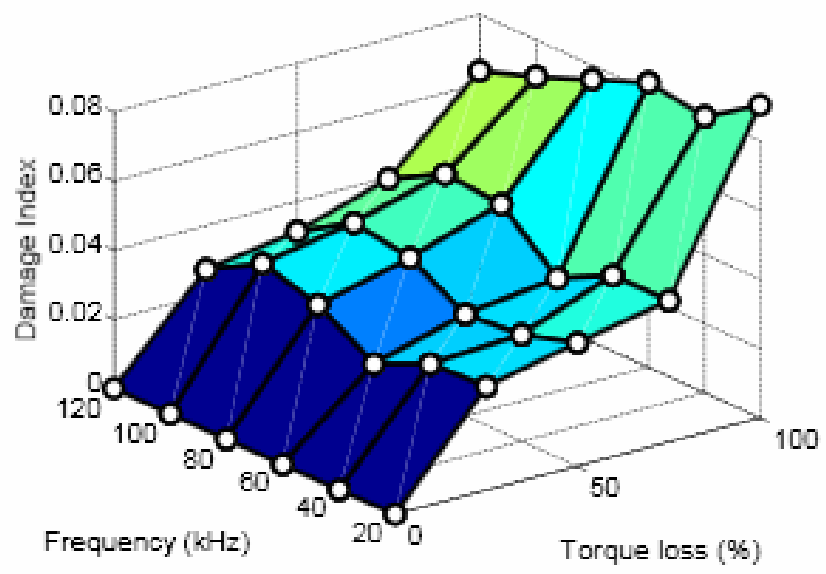

(b) $\mathrm{S} 2$ sensor

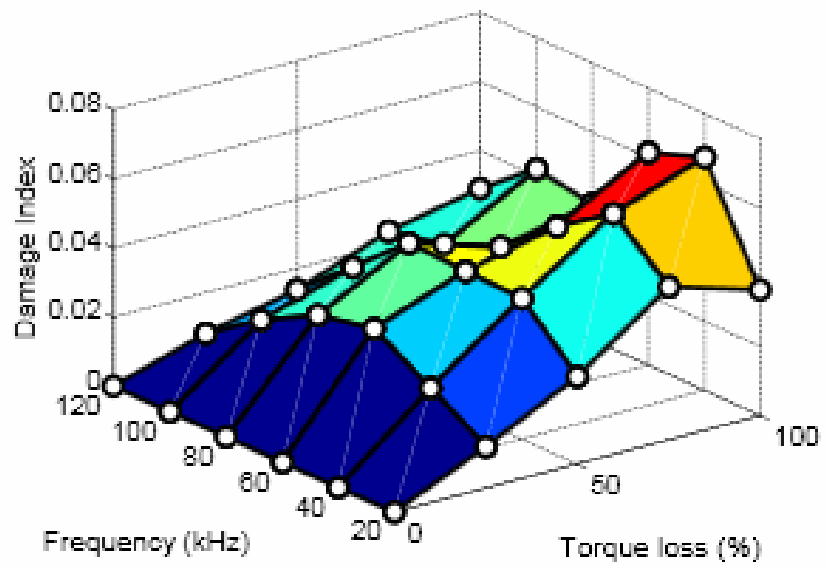

(c) S4 sensor

Figure 3-2-11. Damage index using the energy magnitudes in frequency vs. B1 torque loss and excitation frequency, E3 excitation. 


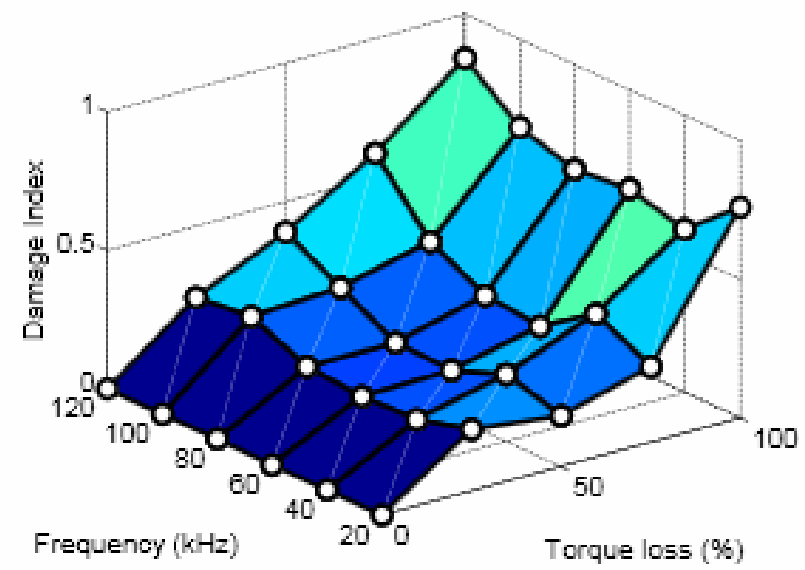

(a) S1 sensor

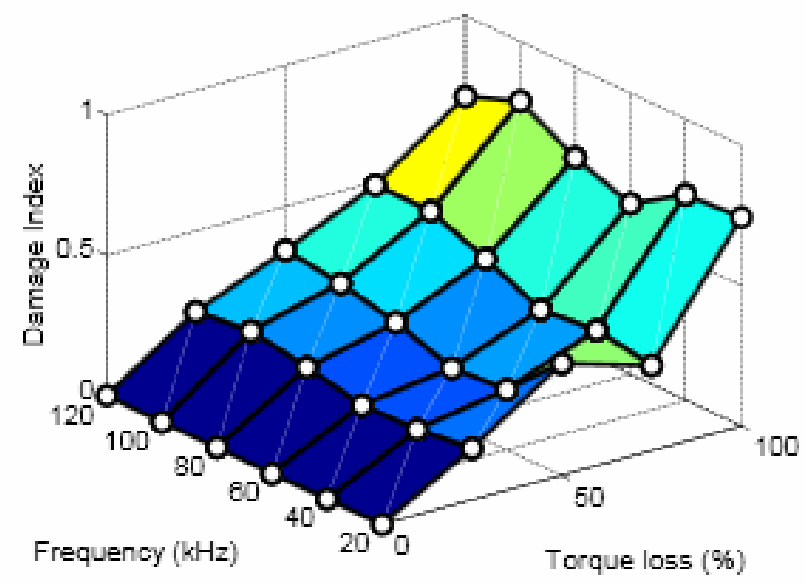

(b) $\mathrm{S} 2$ sensor

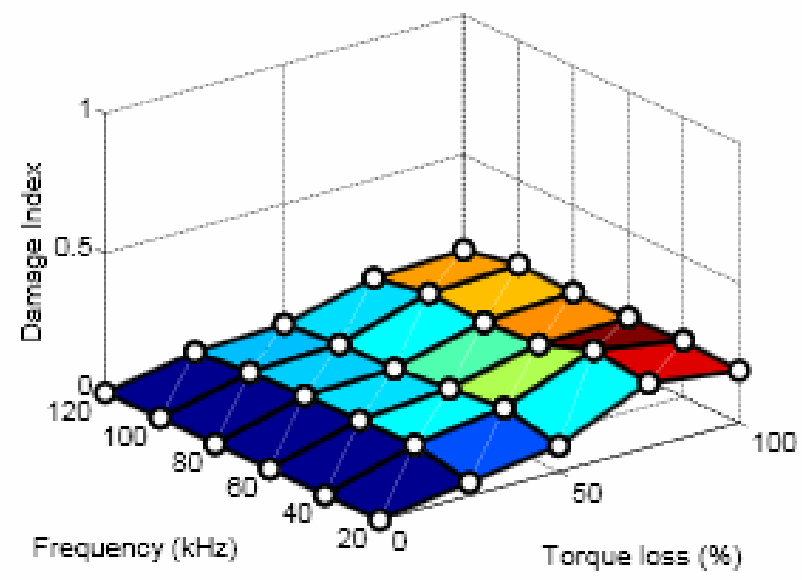

(c) $\mathrm{S} 4$ sensor

Figure 3-2-12. Damage index using the energy magnitudes in frequency vs. B1 torque loss and excitation frequency, E3 excitation. 


\subsubsection{Bolt 1, Excitation Case IV}

Using the piezo-patch in Quadrant 4 (E4) for excitation of the jointed composite panel, sensing information is compiled at the three remaining sensors (S1, S2, S3) located within the remaining three quadrants. In this excitation case, the actuation occurs on the same composite panel (composite 2) as the present damage due to torque loss, but in a different quadrant. Figure 3-2-13 displays the excitation and sensing configuration and indicates the Bolt 1 location of damage due to torque loss. Figure 3-2-14 represents the scattering signal for a $40 \mathrm{kHz}$ excitation frequency under E4 actuation. The signal scattering at S1 and S2 sensors increase in magnitude as torque loss increases. The scattering signals at sensors across the joint from the source of actuation (S1, S2) are smaller in magnitude than the signal from the sensor that shares a composite panel with the source of actuation (S3).

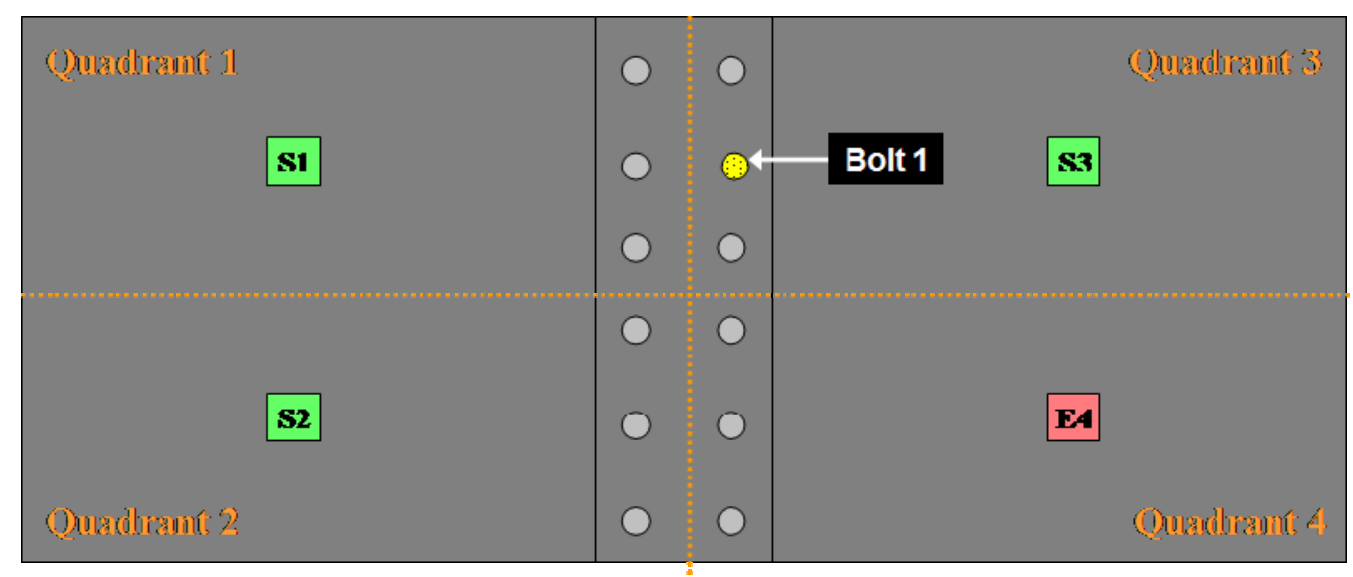

Figure 3-2-13. Excitation Case IV, variable Bolt 1 torque loss. 


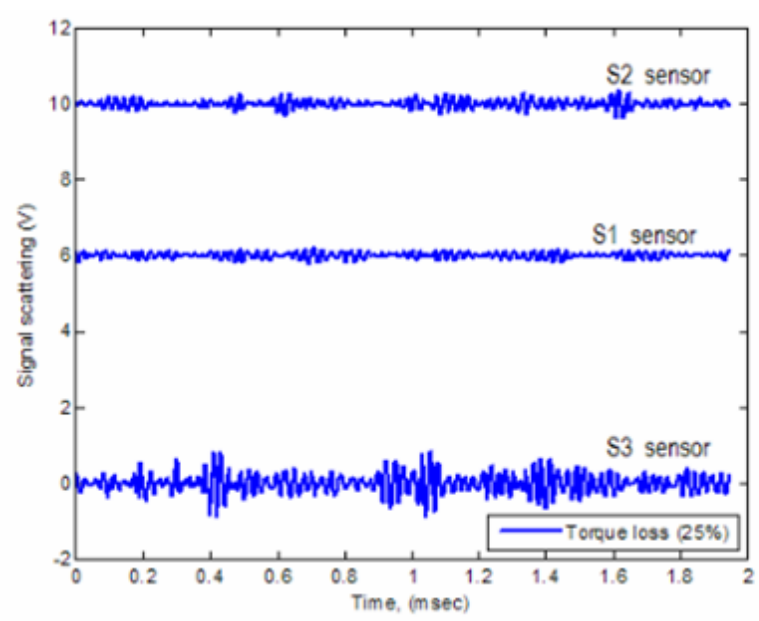

a) $25 \%$ torque loss

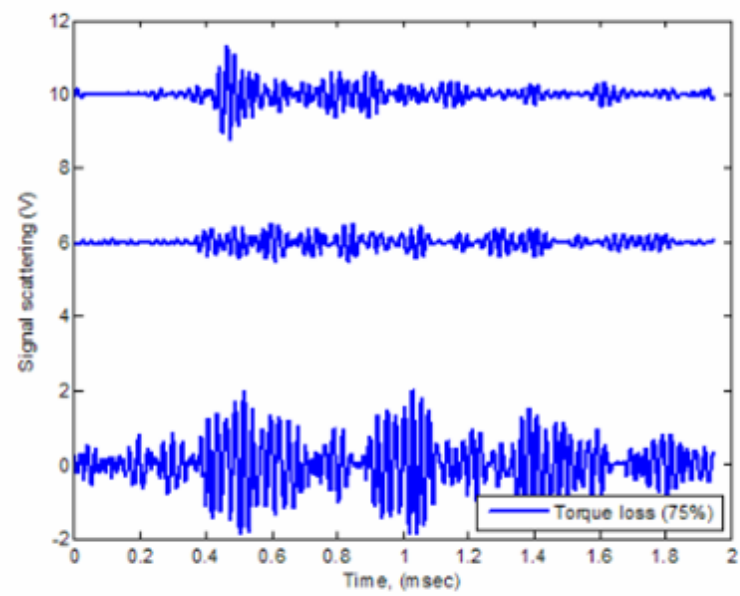

c) $75 \%$ torque loss

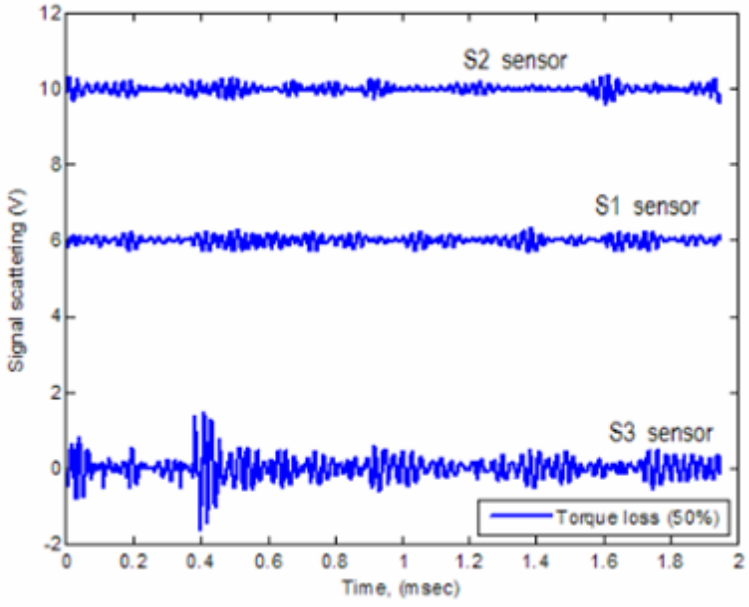

b) $50 \%$ torque loss

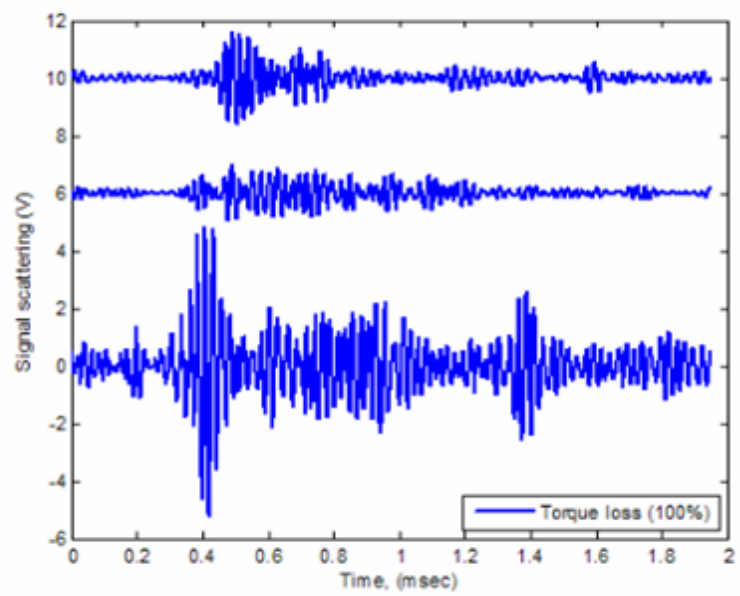

d) $100 \%$ torque loss

Figure 3-2-14. Scattering signals, B1 torque loss, E4 $40 \mathrm{kHz}$ excitation.

Figure 3-2-15 and Figure 3-2-16 present the damage index using the energy magnitudes due to frequency response $\left(D I_{f}\right)$ and using the signals in time $\left(D I_{t}\right)$ at $\mathrm{S} 1, \mathrm{~S} 2$ and S3 under an E4 excitation case. Even when actuation is performed on the same composite panel (composite 2) where damage is present, the damage detection capabilities of each sensor using time and frequency response magnitudes are favorable under the E4 excitation configuration with respect to Bolt 1 torque loss. 


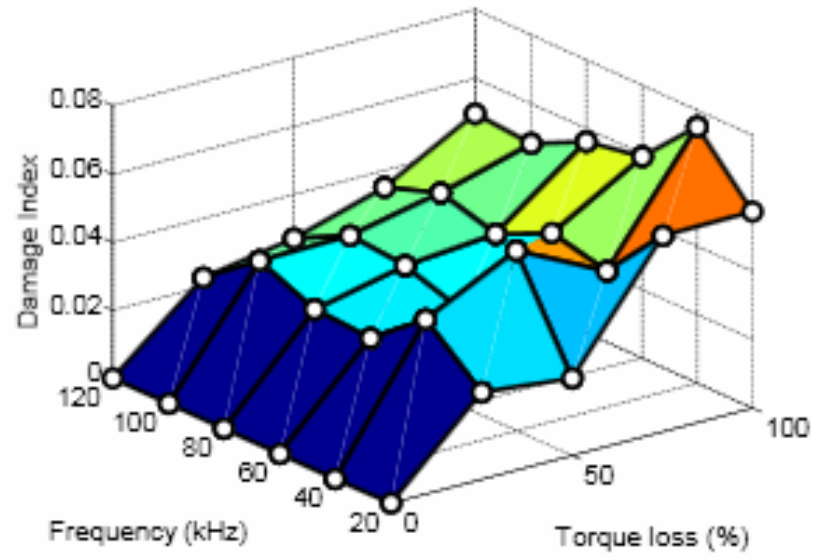

(a) $\mathrm{S} 1$ sensor

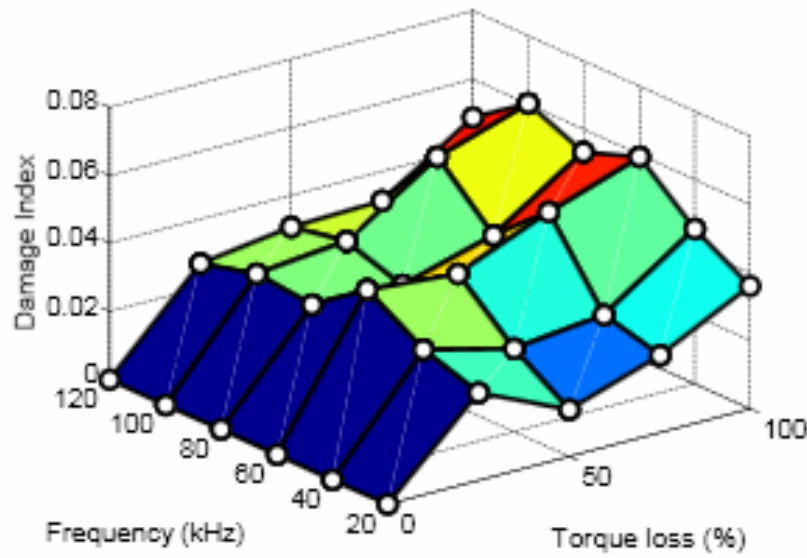

(b) $\mathrm{S} 2$ sensor

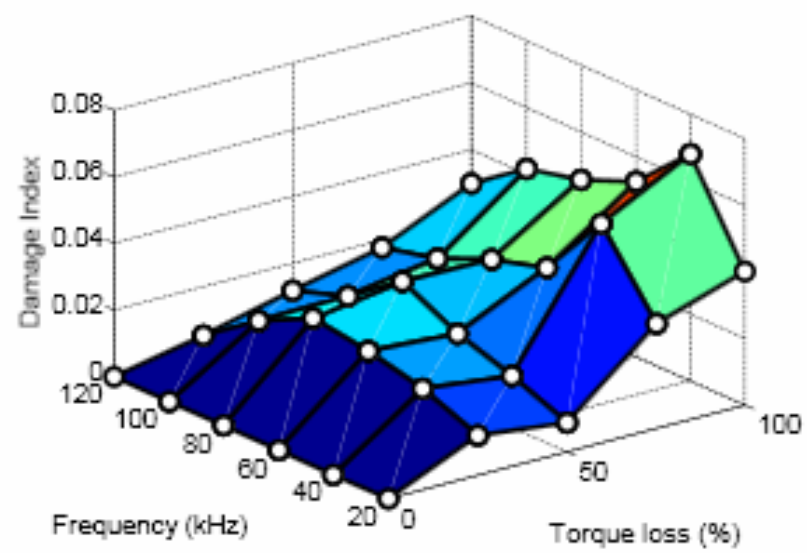

(c) $\mathrm{S} 3$ sensor

Figure 3-2-15. Damage index using the energy magnitudes in frequency vs. B1 torque loss and excitation frequency, E4 excitation. 


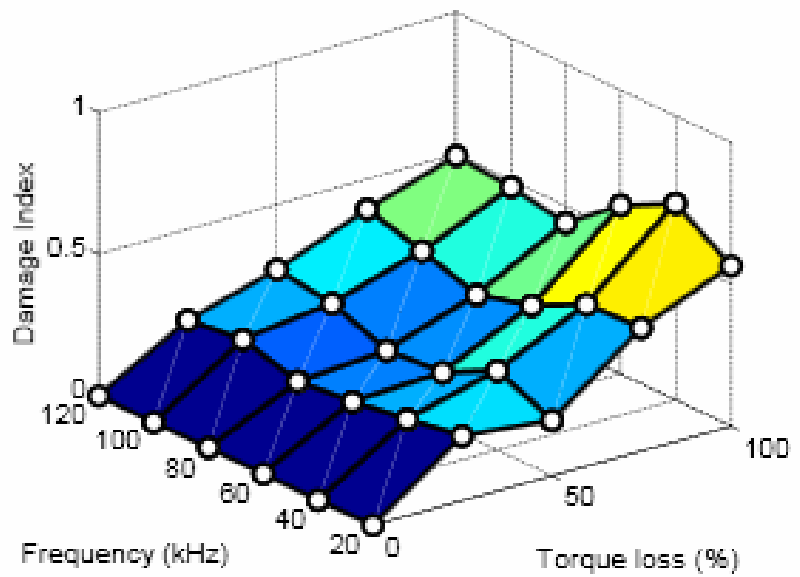

(a) $\mathrm{S} 1$ sensor

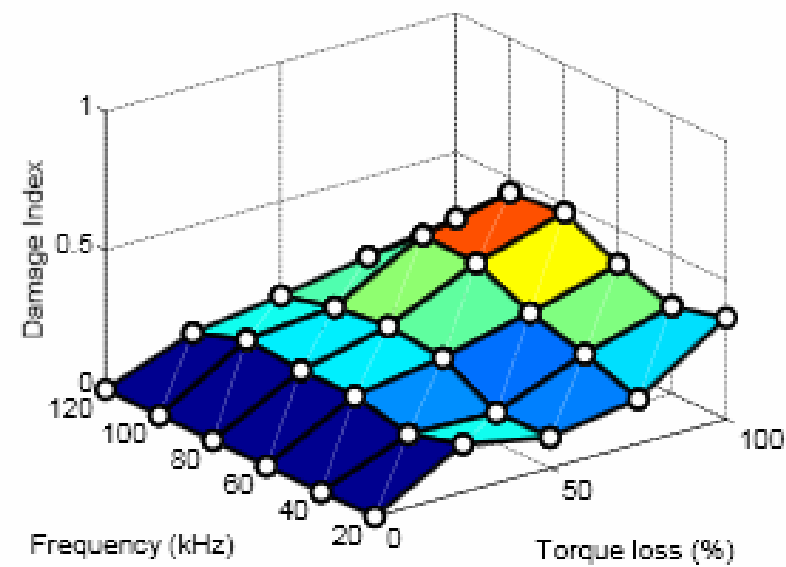

(b) $\mathrm{S} 2$ sensor

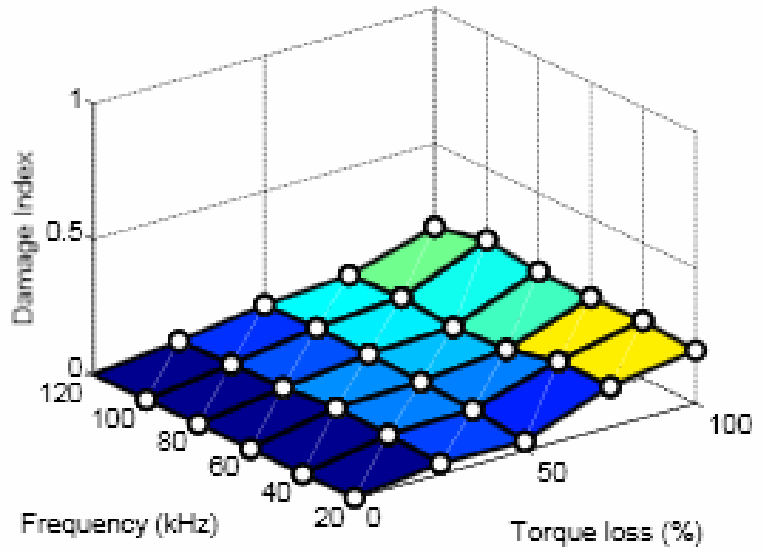

(c) S3 sensor

Figure 3-2-16. Damage index using the energy magnitudes in frequency vs. B1 torque loss and excitation frequency, E4 excitation. 


\subsubsection{Comparison of Bolt 1 excitation configurations}

Figure 3-2-17 shows the side-by-side comparison of the normalized average damage index of the composite jointed panel due to Bolt 1 torque loss for each excitation configuration case (E1, E2, E3 and E4). Similar to the results of Bolt 0 torque loss (Figure 3-1-33), the damage detection capability provided insight into the presence and location of damage due to bolt torque loss. To provide a rough estimate of the location of damage due to torque loss within the jointed composite panel, the following analysis is made:

$$
\begin{array}{ll}
D I_{E 1, S 3} \geq D I_{E 1, S 4}>>D I_{E 1, S 2} & \text { Figure 3-2-17 (a) } \\
D I_{E 2, S 3}>D I_{E 2, S 4}>>D I_{E 2, S 1} & \text { Figure 3-2-17 (b) } \\
D I_{E 3, S 1} \approx D I_{E 3, S 2}>>D I_{E 3, S 4} & \text { Figure 3-2-17 (c) } \\
D I_{E 4, S 1}>D I_{E 4, S 2}>>D I_{E 4, S 3} & \text { Figure 3-2-17 (d) }
\end{array}
$$

The comparison of average damage index trends over each excitation case indicates that the damage due to torque loss is somewhere within the area between sensors S1 and S3, thus within Quadrant 1 or Quadrant 3. The average damage index $D I_{E 3, S 1}$ and $D I_{E 3, S 2}$ are nearly indistinguishable among the percentage torque loss values. 


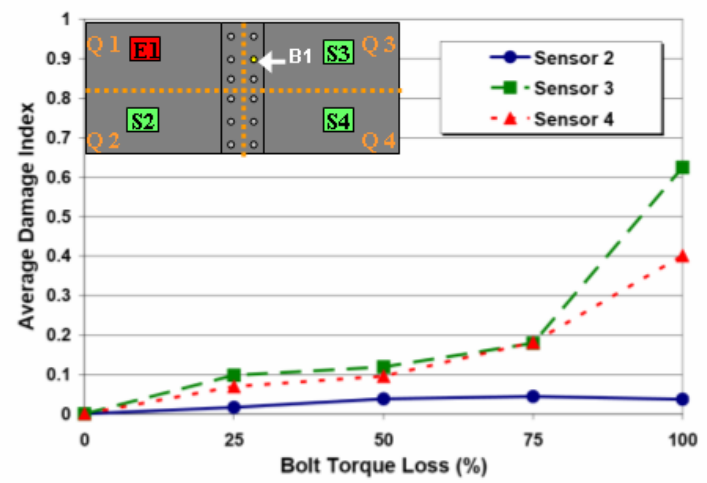

(a) E1 excitation

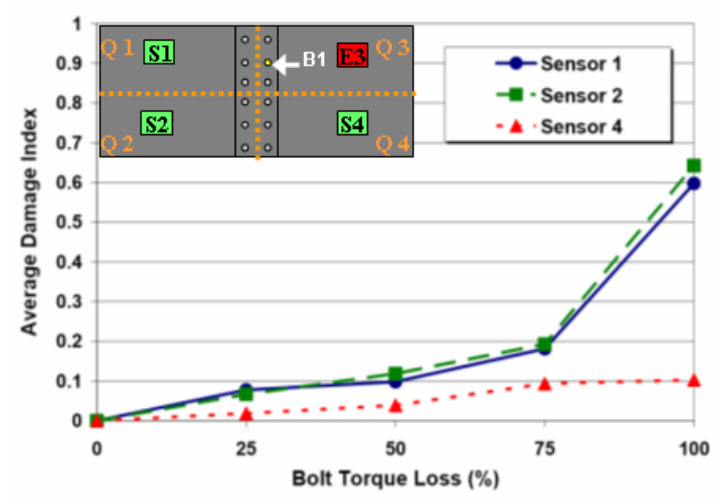

(c) E3 excitation

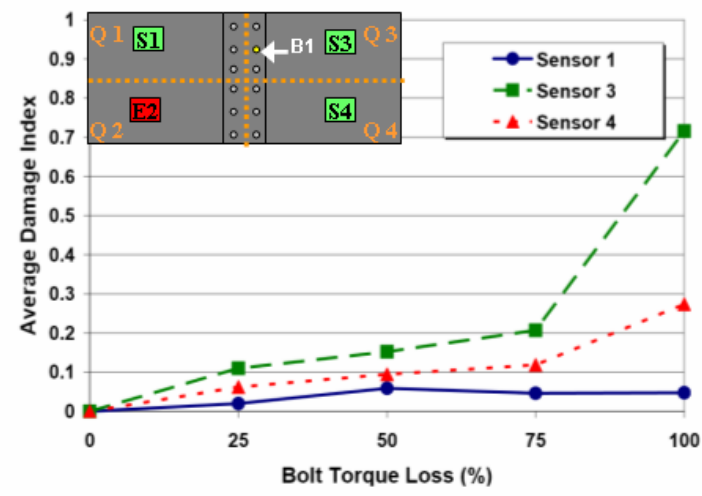

(b) E2 excitation

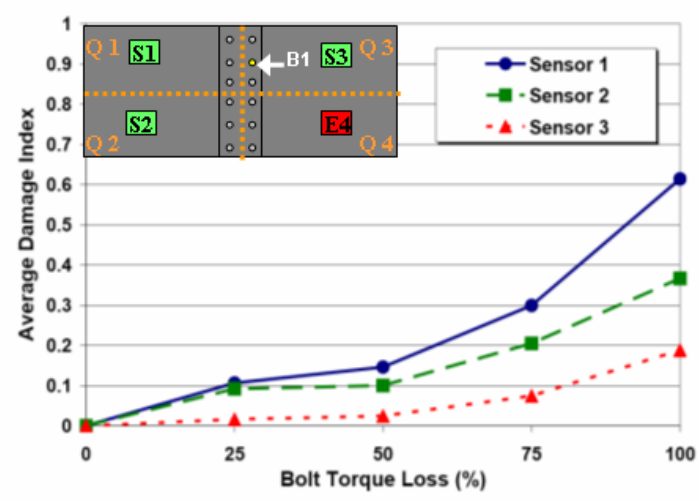

(d) E4 excitation

Figure 3-2-17. Comparison of normalized average damage index, B1 torque loss.

Similar to the analysis of Bolt 0 average damage index in section 3.1.5, the clearest distinction of data for a possible damage location in-between sensors S1 and S3 comes from the E2 and E4 excitation cases. When actuation is present in quadrants furthest from the predicted damage areas, the sensing information provides great insight toward the localization of damage within the composite jointed panel. Figure 3-2-18 shows the graphical comparison of the $D I_{E 4, S I}$ and $D I_{E 2, S 3}$ taken from Figure 3-2-17 (b) and Figure 3-2-17 (d). 


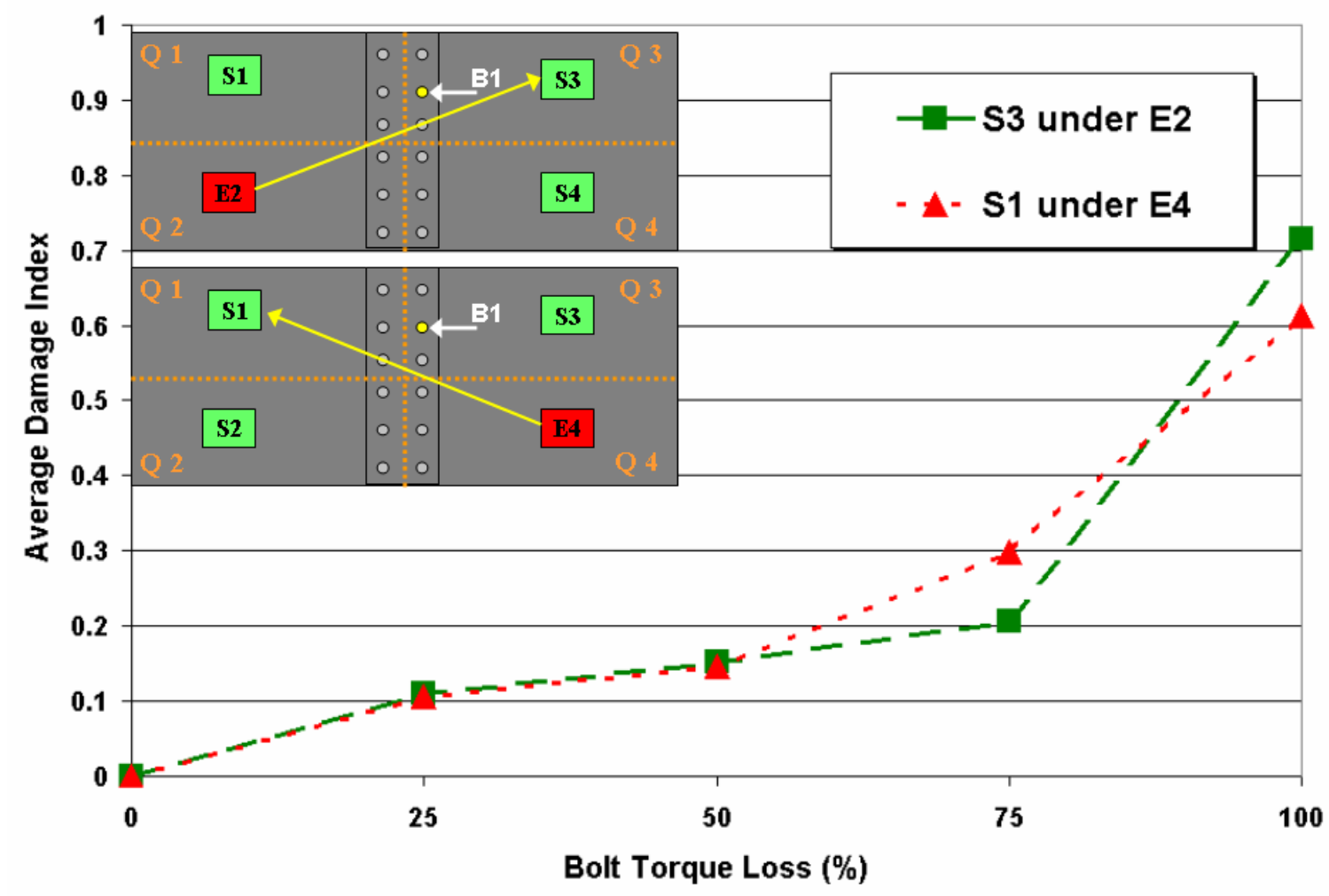

Figure 3-2-18. Comparison of normalized average damage indices, $\mathrm{B} 1$ torque loss; $D I_{E 4, S I}$ and $D I_{E 2, S 3}$.

As compared to the Bolt 0 average damage index values for S3 and S1 information in Figure 3-2-18, the information present for damage due to torque loss in Bolt 1 is not as clear. However, a trend still remains. There is a clear difference between $D I$ at $75 \%$ torque loss which does not correlate with resulted expected from a similar analysis performed on Bolt 0 information. At $25 \%$ and $50 \%$ torque loss, $D I_{E 2, S 3}$ was only slightly higher than $D I_{E 4, S I}$. At $100 \%$ torque loss, $D I_{E 2, S 3}$ was clearly higher than $D I_{E 4, S I}$. This demonstration raises the question as to whether damage quantification does affect the level of confidence behind damage localization using this method. Given that three of the four inflicted damage levels indicate a damage position closest to S3, 
Figure 3-2-19 shows the shaded area of the highest likelihood of damage to be within the joint area located in Quadrant 3.

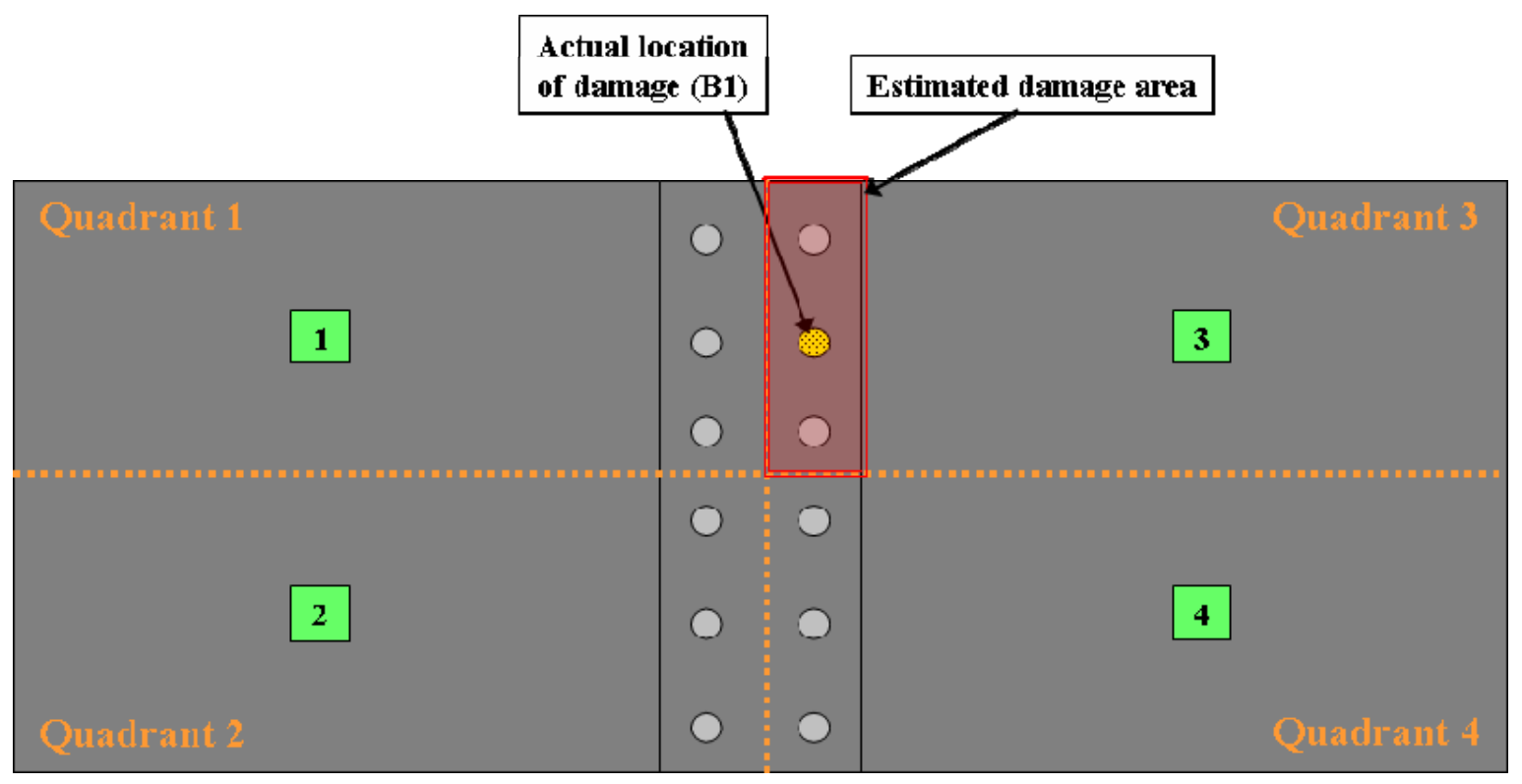

Figure 3-2-19. Estimated damage area due to B1 torque loss.

\subsection{Bolt 2 damage due to torque loss}

In this section, the torque of Bolt 2 is manipulated in the same manner as Bolt 0 and Bolt 1 to evaluate the damage detection capabilities of the smart sensor array system on the jointed composite panel. In this case, the damaged bolt was further toward the center of the composite panel, and borders Quadrant 2. Five torque values were tested at each of the four excitation configuration cases. Experimental results were compiled exactly as the Bolt 0 and Bolt 1 phases of experimentation, and monitored closely to determine if the bolt's proximity to Quadrant 2 would affect the trends noticed from Bolts 0 and 1. 


\subsubsection{Bolt 2, Excitation Case I}

Using the piezo-patch in Quadrant 1 (E1) for excitation of the jointed composite panel, sensing information is compiled at the three remaining sensors (S2, S3, and S4). Figure 3-3-1 shows the excitation and sensing configuration and indicates the Bolt 2 location of simulated damage. Figure 3-3-2 represents the scattering signal for a $40 \mathrm{kHz}$ excitation frequency under E1 actuation. The signal scattering at S3 and S4 sensors increase in magnitude as torque loss increases. Due to the incident signals loss as it passes through the composite joint, the scattering signals at sensors S3 and S4 are smaller in magnitude than the signal from sensor S2.

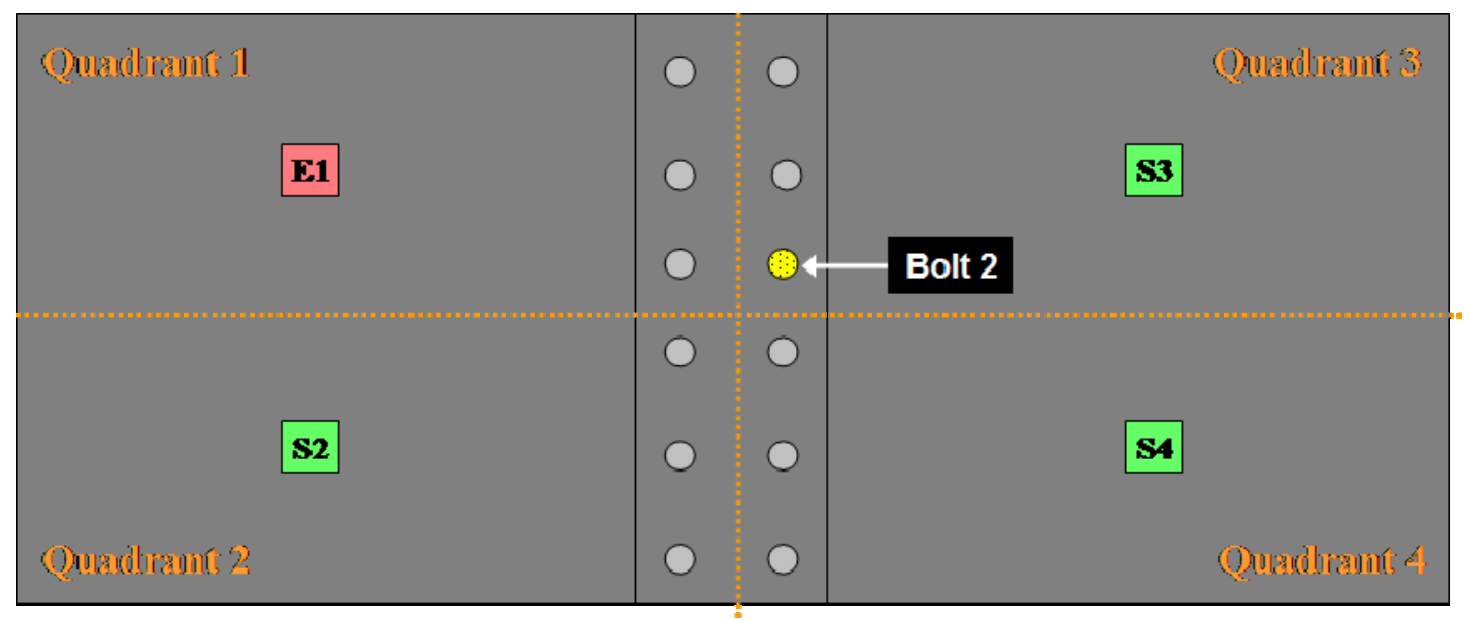

Figure 3-3-1. Excitation Case I, variable Bolt 2 torque loss.

Figure 3-3-3 presents the damage index using the energy magnitudes due to frequency response $\left(D I_{f}\right)$ at $\mathrm{S} 2, \mathrm{~S} 3$ and $\mathrm{S} 4$ under E1 excitation. Figure 3-3-4 presents the damage index using the signals in time $\left(D I_{t}\right)$ at $\mathrm{S} 2, \mathrm{~S} 3$ and $\mathrm{S} 4$ sensing locations. In each plot, the damage index is more sensitive to changes in damage level as compared to changes in excitation frequency. 


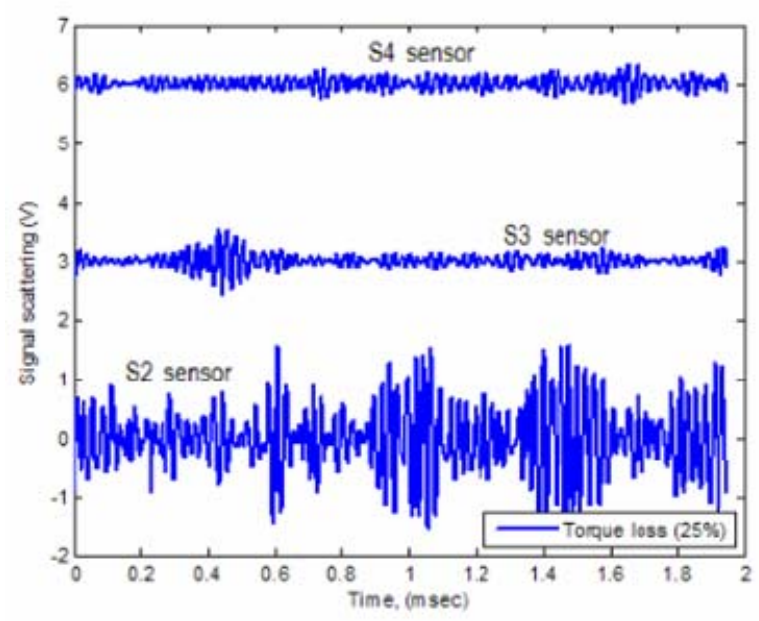

a) $25 \%$ torque loss

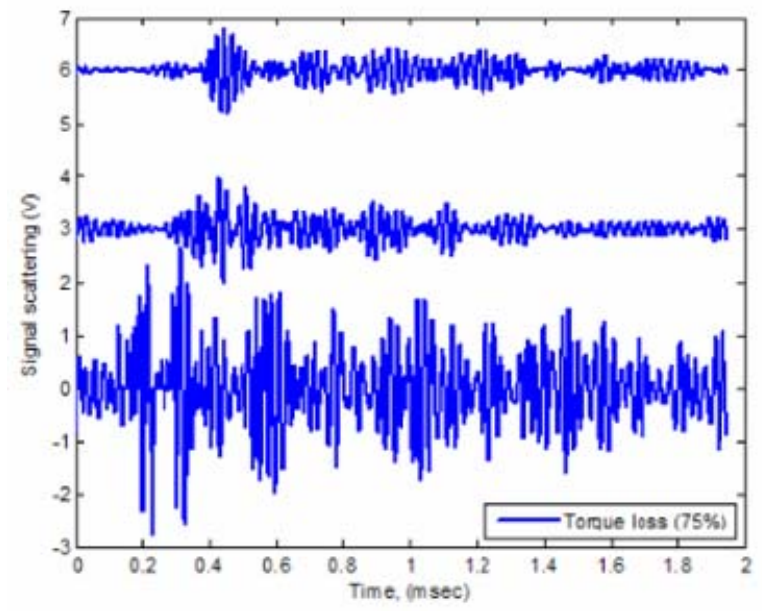

c) $75 \%$ lorque loss

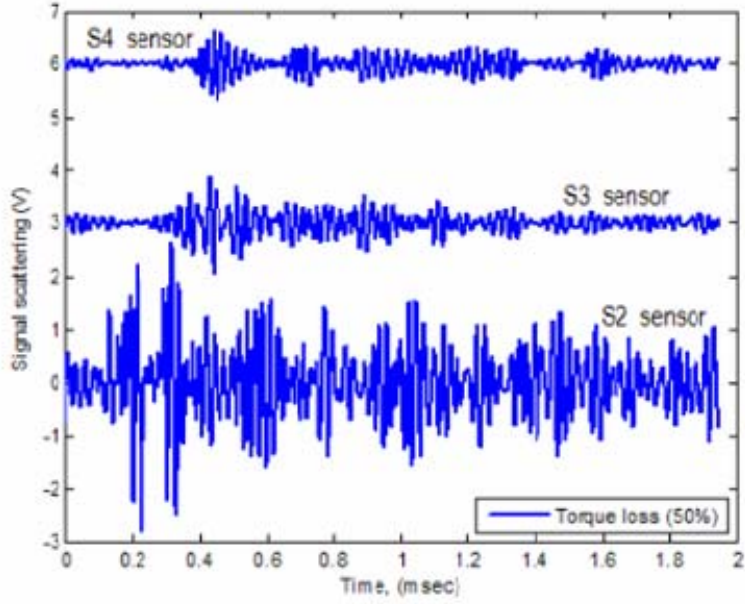

b) $50 \%$ torque loss

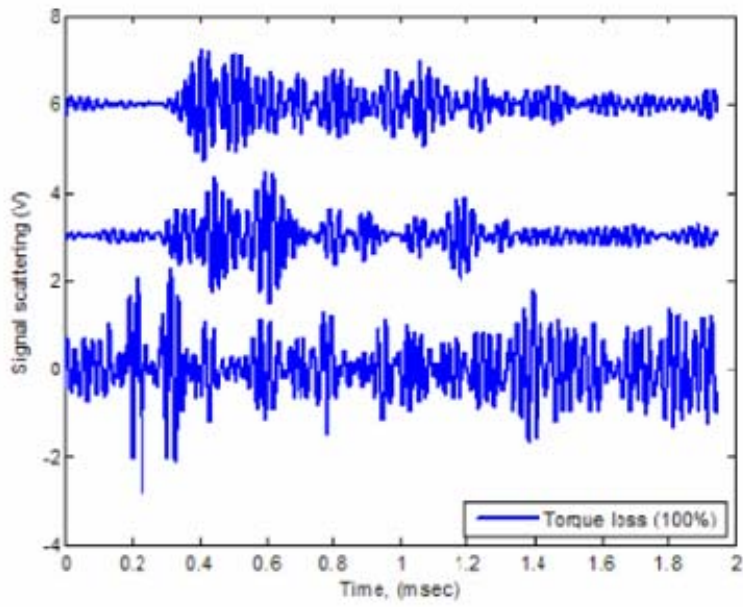

d) $100 \%$ torque loss

Figure 3-3-2. Scattering signals, B2 torque loss, E1 $40 \mathrm{kHz}$ excitation. 


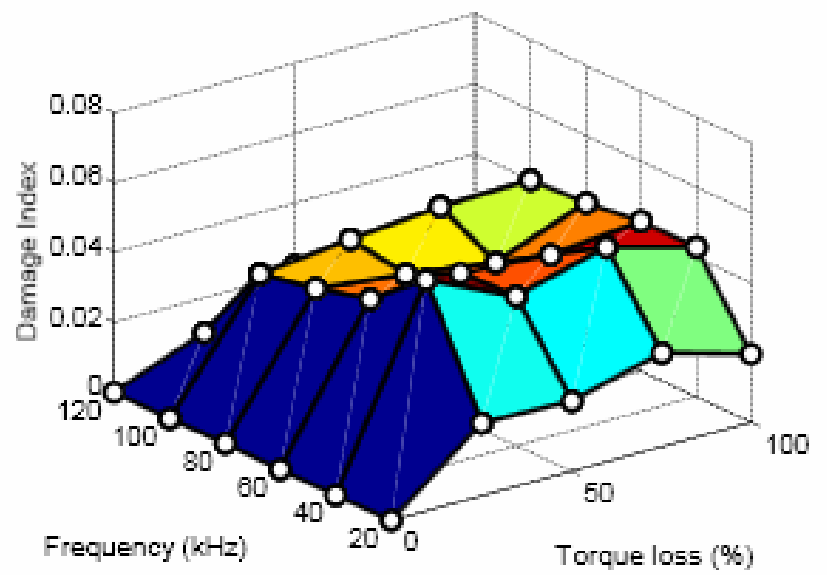

(a) $\mathrm{S} 2$ sensor

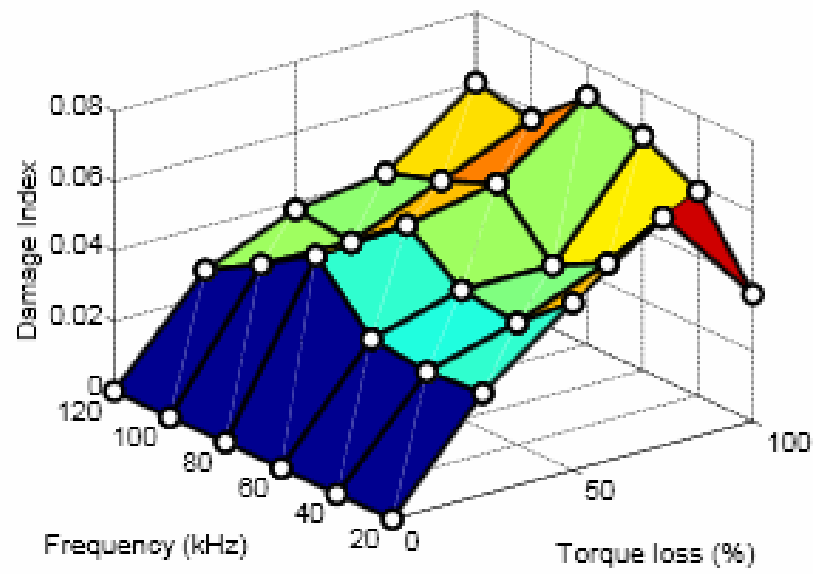

(b) $\mathrm{S} 3$ sensor

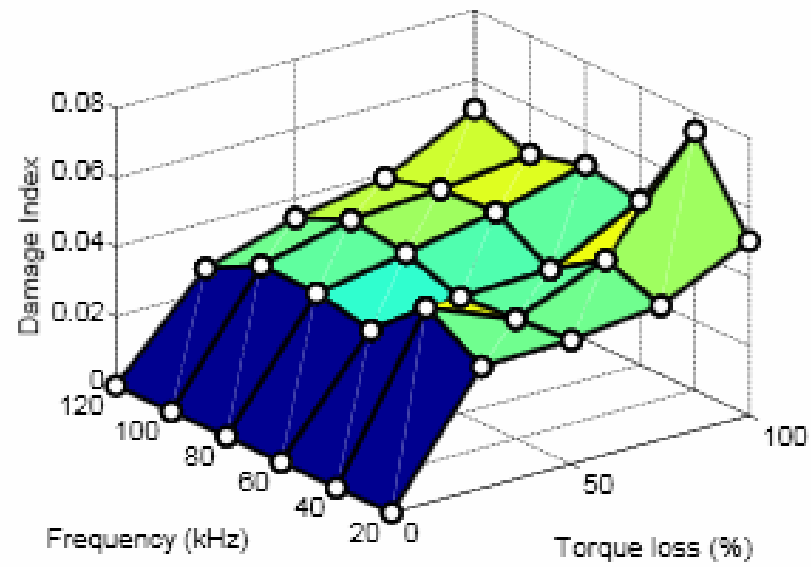

(c) $\mathrm{S} 4$ sensor

Figure 3-3-3. Damage index using the energy magnitudes in frequency vs. B2 torque loss and excitation frequency, E1 excitation. 


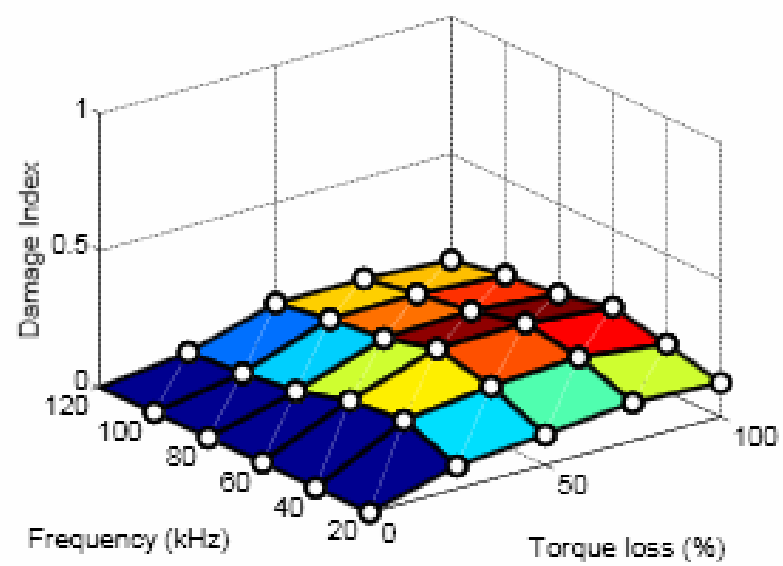

(a) $\mathrm{S} 2$ sensor

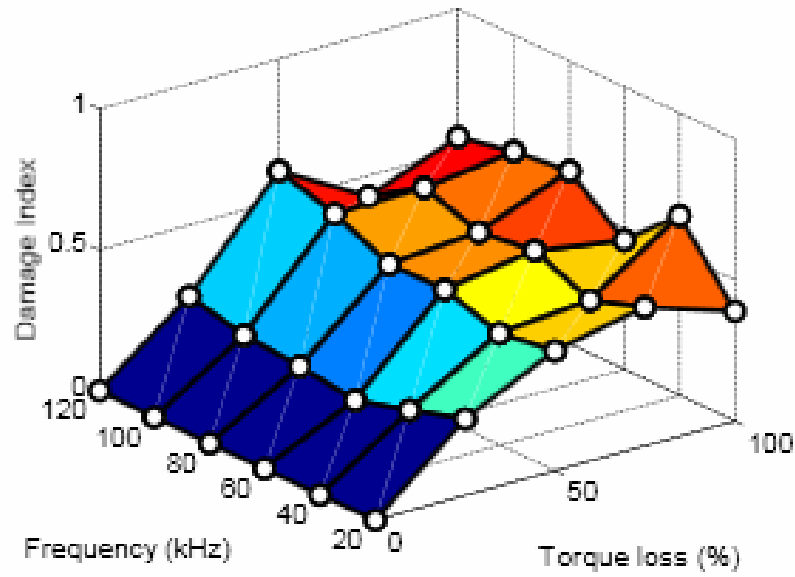

(b) $\mathrm{S} 3$ sensor

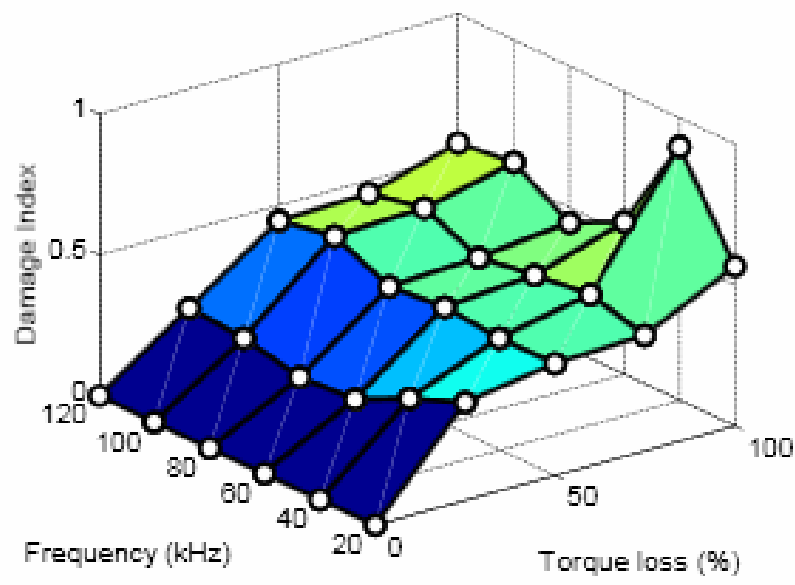

(c) S4 sensor

Figure 3-3-4. Damage index using the energy magnitudes in frequency vs. B2 torque loss and excitation frequency, E1 excitation. 


\subsubsection{Bolt 2, Excitation Case II}

Using the piezo-patch in Quadrant 2 (E2) for excitation of the jointed composite panel, sensing information is compiled at the three remaining sensors (S1, S3, S4) located within the remaining three quadrants. Figure 3-3-5 displays the excitation and sensing configuration and indicates the Bolt 2 location of simulated damage. Figure 3-3-6 represents the scattering signal for a $40 \mathrm{kHz}$ excitation frequency under E2 actuation. The signal scattering at S3 and S4 sensors increase in magnitude as torque loss increases. The scattering signals at sensors across the joint from the source of actuation (S3, S4) are smaller in magnitude than the signal from the sensor that shares a composite panel with the source of actuation (S1).

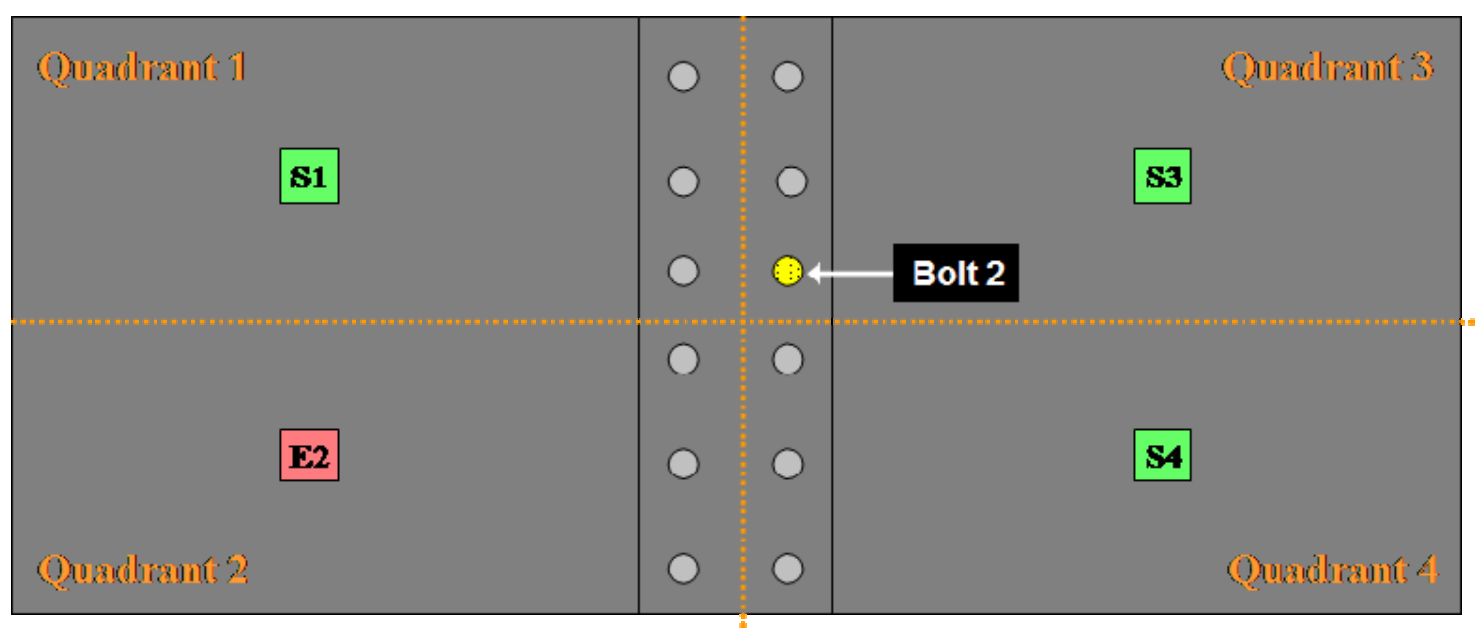

Figure 3-3-5. Excitation Case II, variable Bolt 2 torque loss. 


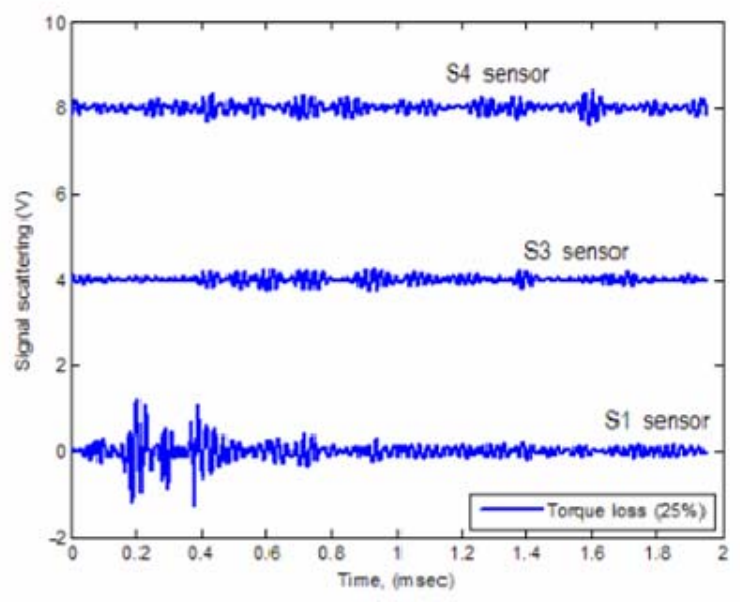

a) $25 \%$ torque $\operatorname{loss}$

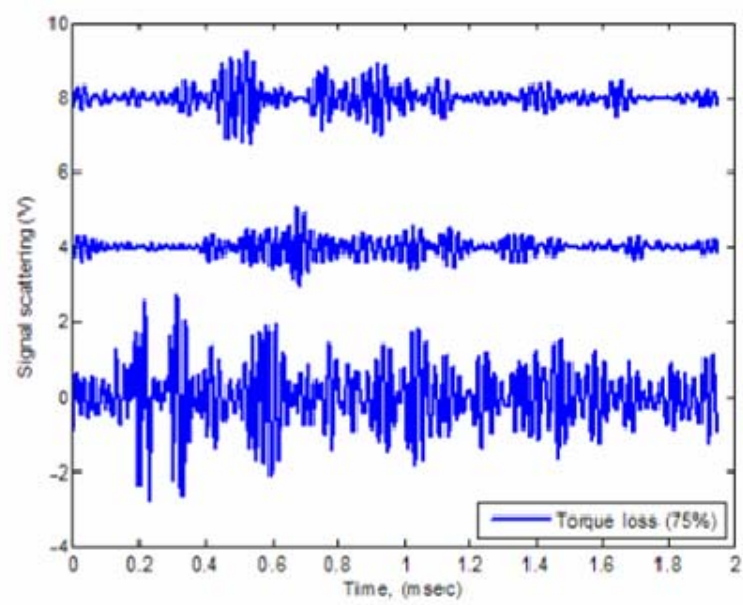

c) $75 \%$ torque loss

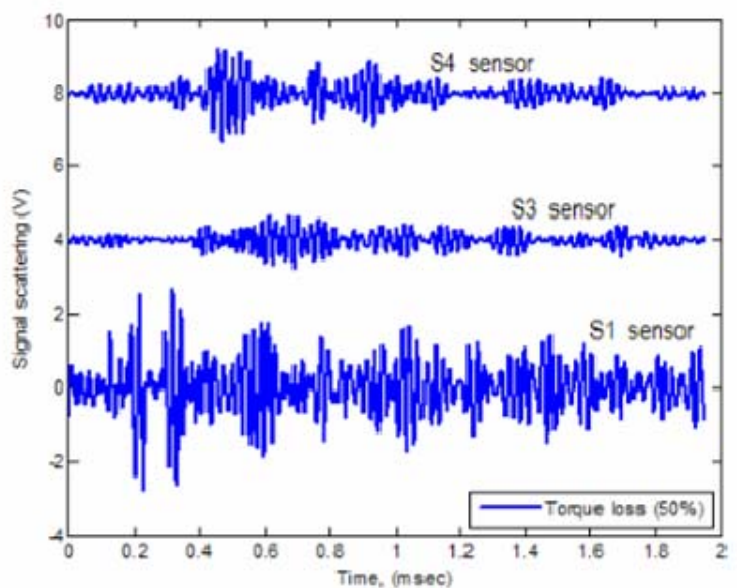

b) $50 \%$ torque loss

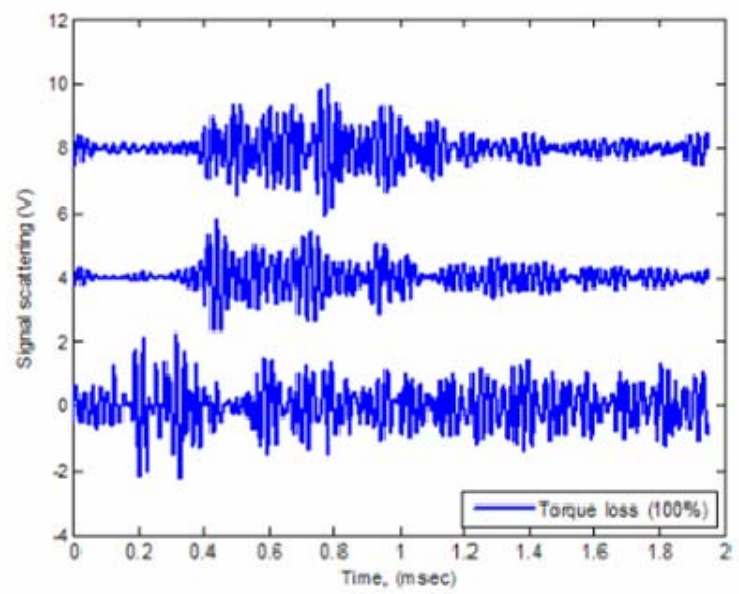

d) $100 \%$ torque loss

Figure 3-3-6. Scattering signals, B2 torque loss, E2 $40 \mathrm{kHz}$ excitation frequency. 


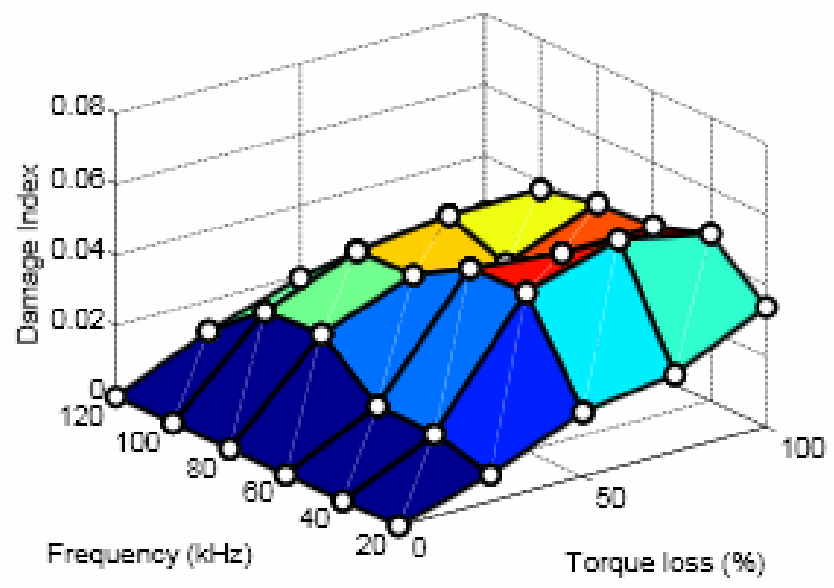

(a) $\mathrm{S} 1$ sensor

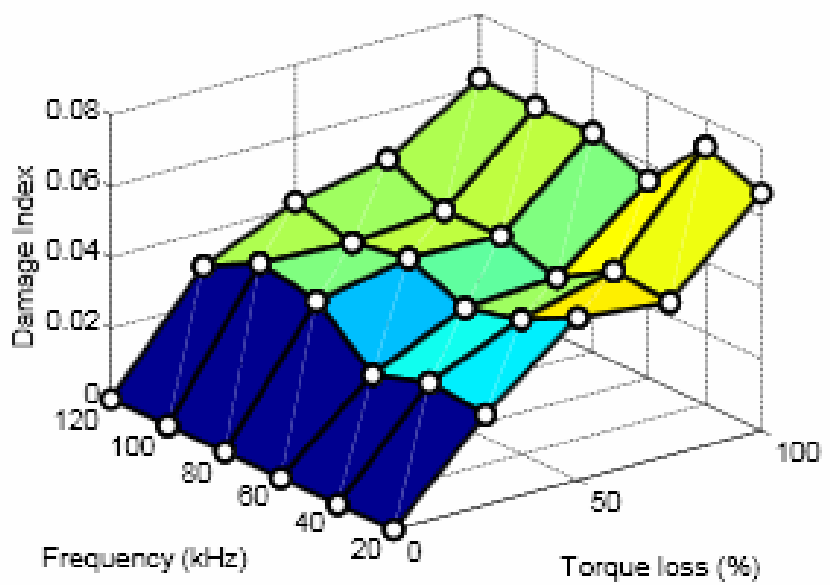

(b) $\mathrm{S} 3$ sensor

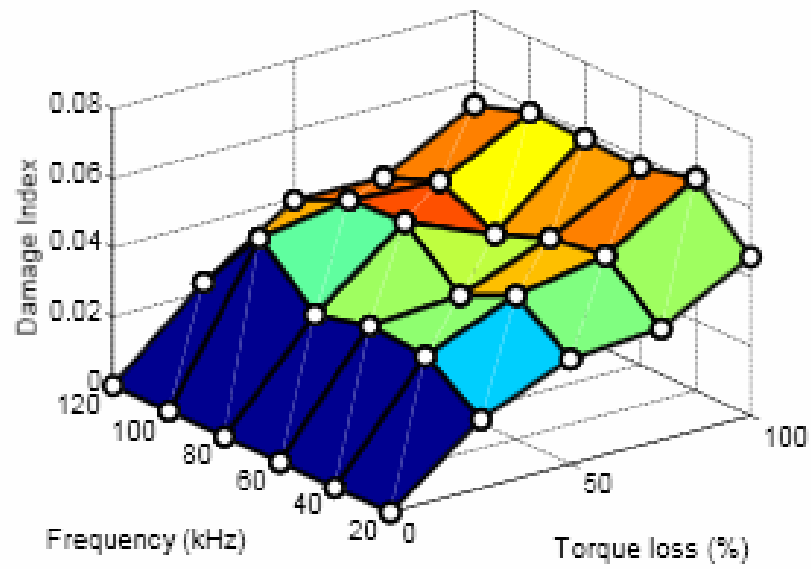

(c) S4 sensor

Figure 3-3-7. Damage index using the energy magnitudes in frequency vs. B2 torque loss and excitation frequency, E2 excitation. 


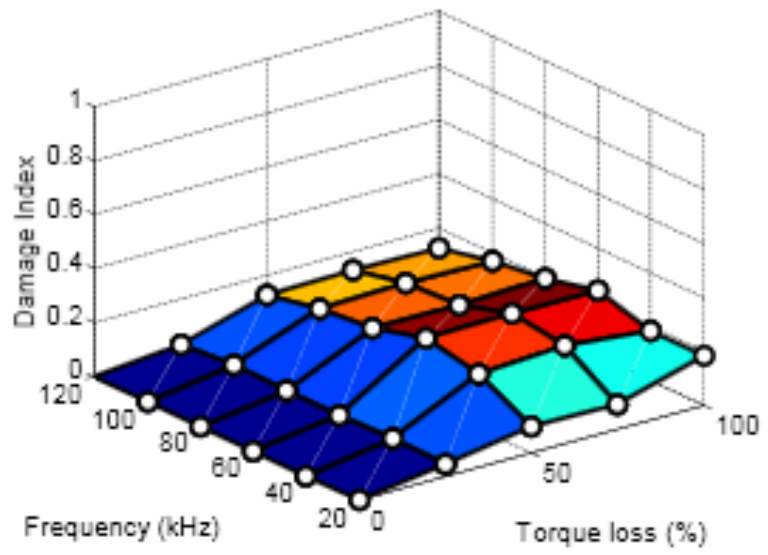

(a) $\mathrm{S} 1$ sensor

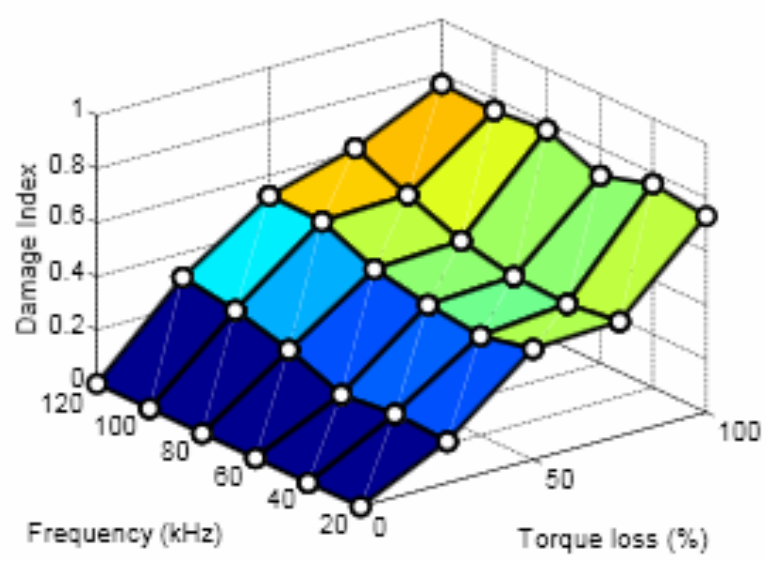

(b) $\mathrm{S} 3$ sensor

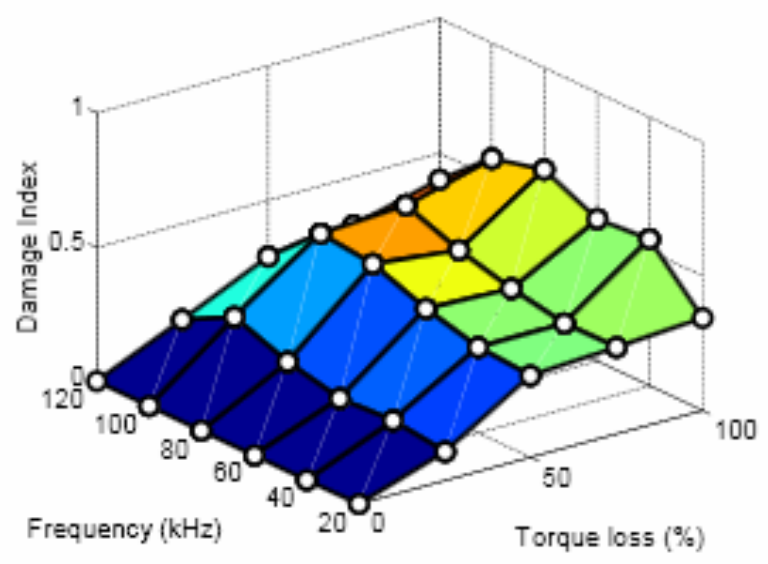

(c) S4 sensor

Figure 3-3-8. Damage index using the signals in time vs. B2 torque loss and excitation frequency, E2 excitation. 


\subsubsection{Bolt 2, Excitation Case III}

Using the piezo-patch in Quadrant 3 (E3) for excitation of the jointed composite panel, sensing information is compiled at the three remaining sensors (S1, S3, S4) located within the remaining three quadrants. In this scenario, the actuation occurred in the same quadrant where damage was present due to torque loss. Figure 3-3-9 displays the excitation and sensing configuration and indicates the Bolt 2 location of simulated damage. Figure 3-3-10 represents the scattering signal for a $40 \mathrm{kHz}$ excitation frequency under E3 actuation. The signal scattering at S1 and S2 sensors increase in magnitude as torque loss increases. The scattering signals at sensors across the joint from the source of actuation $(\mathrm{S} 1, \mathrm{~S} 2)$ are smaller in magnitude than the signal from the sensor that shares a composite panel with the source of actuation (S4).

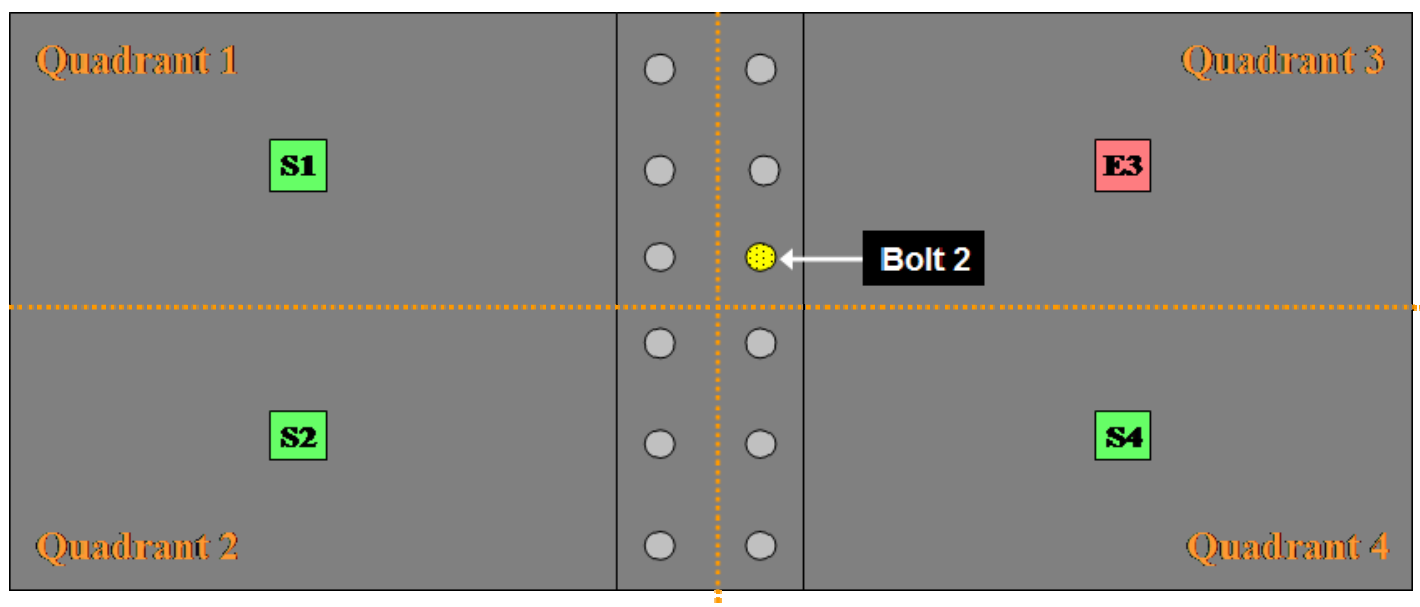

Figure 3-3-9. Excitation Case III, variable Bolt 2 torque loss.

Figure 3-3-11 presents the damage index using the energy magnitudes due to frequency response $\left(D I_{f}\right)$ at S1, S2 and S4 under E3 excitation. Figure 3-3-12 presents the damage index using the signals in time $\left(D I_{t}\right)$ at $\mathrm{S} 1, \mathrm{~S} 2$ and $\mathrm{S} 4$ sensing locations. In 
each plot, the damage index displays a higher sensitivity to increased bolt torque loss and a lesser sensitivity over the range of excitation frequencies.

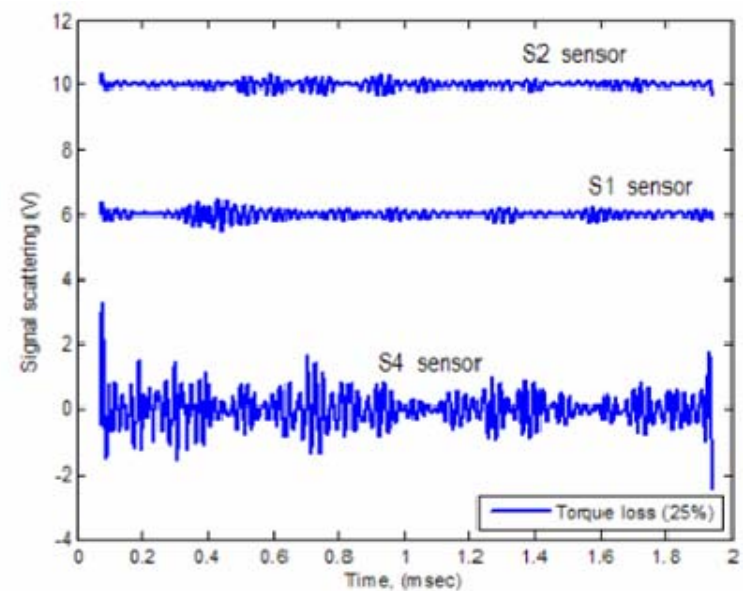

a) $25 \%$ torque loss

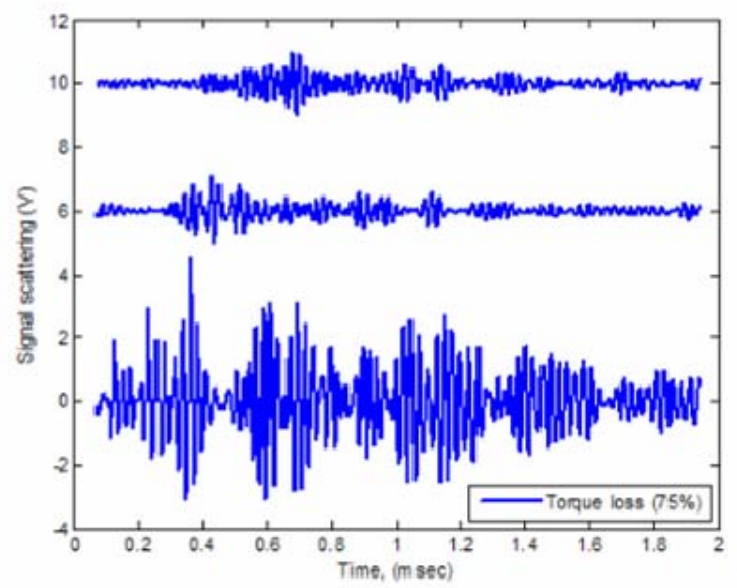

c) $75 \%$ torque loss

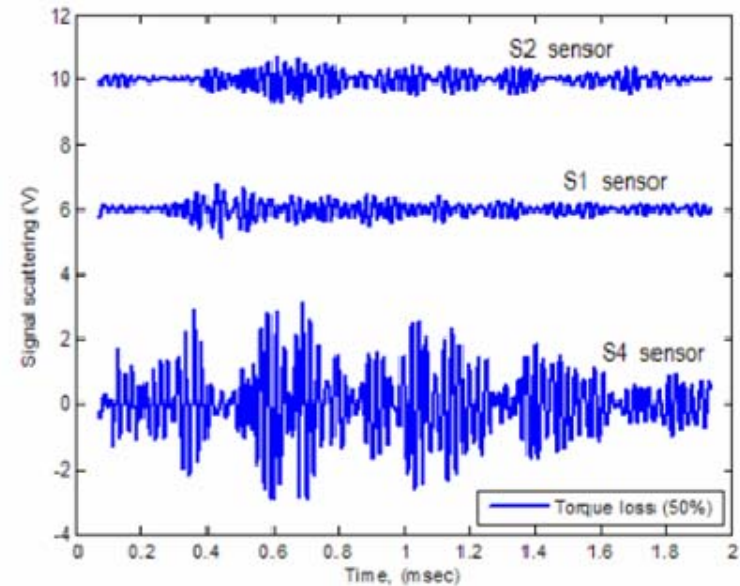

b) $50 \%$ torque loss

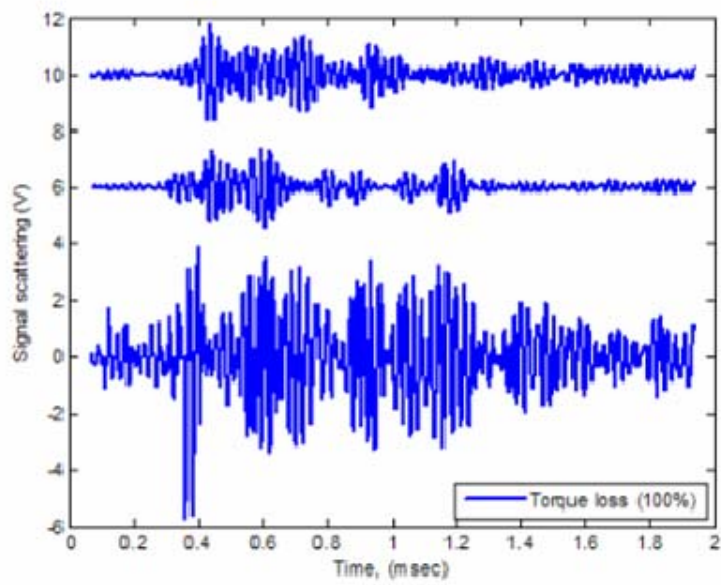

d) $100 \%$ torque loss

Figure 3-3-10. Scattering signals, B2 torque loss, E3 $40 \mathrm{kHz}$ excitation. 


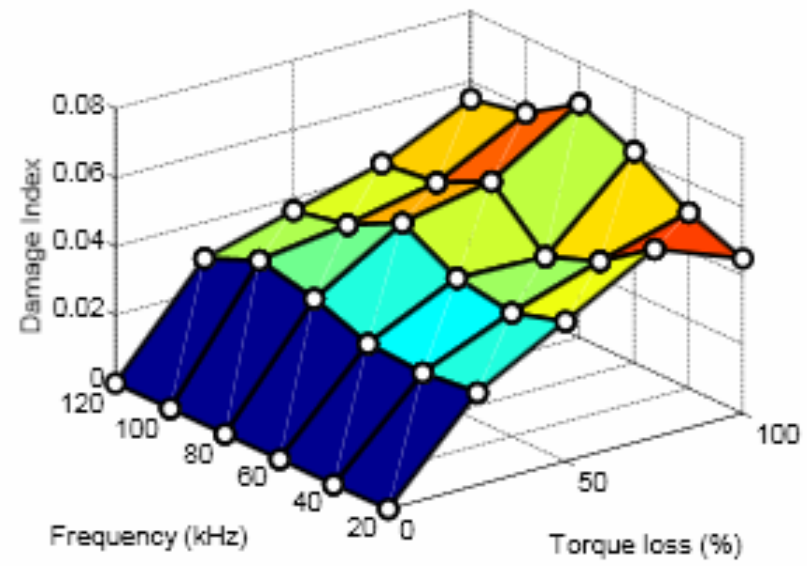

(a) $\mathrm{S} 1$ sensor

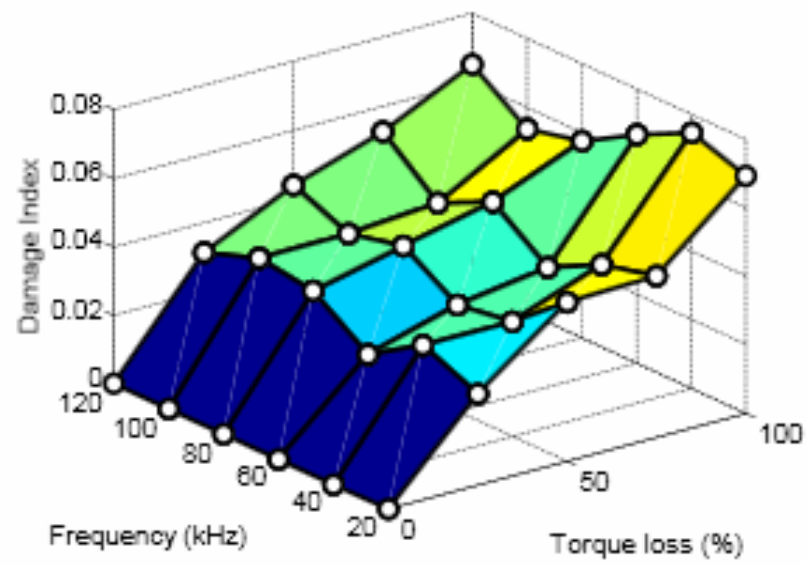

(b) $\mathrm{S} 2$ sensor

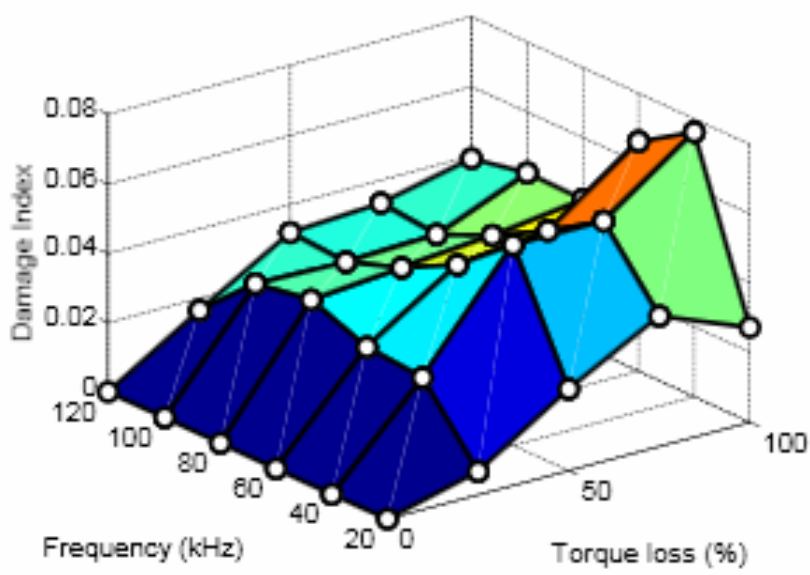

(c) $\mathrm{S} 4$ sensor

Figure 3-3-11. Damage index using the energy magnitudes in frequency vs. B2 torque loss and excitation frequency, E3 excitation. 


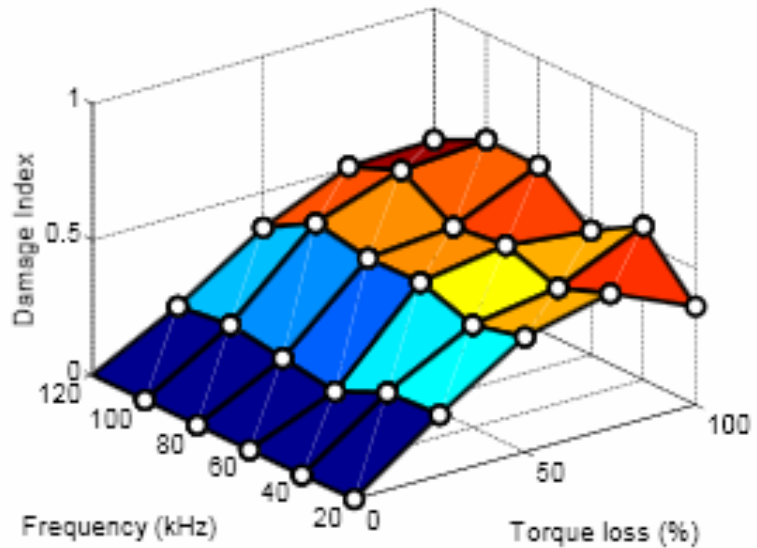

(a) S1 sensor

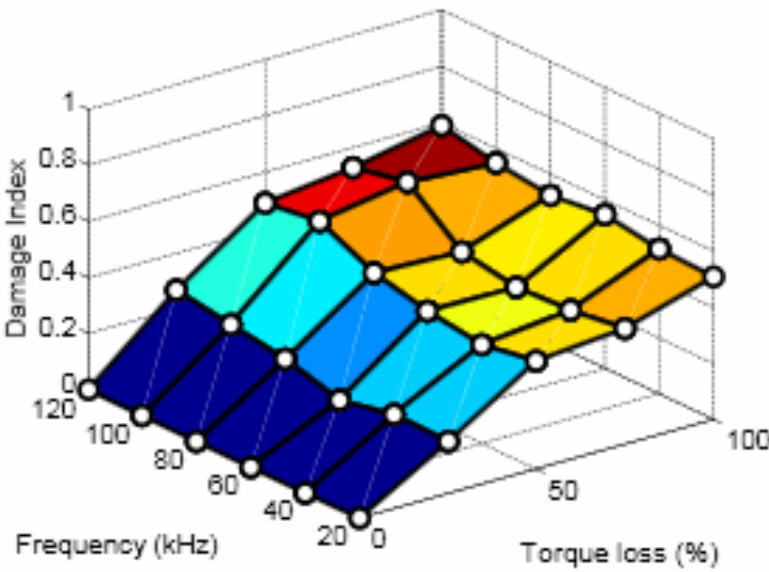

(b) S2 sensor

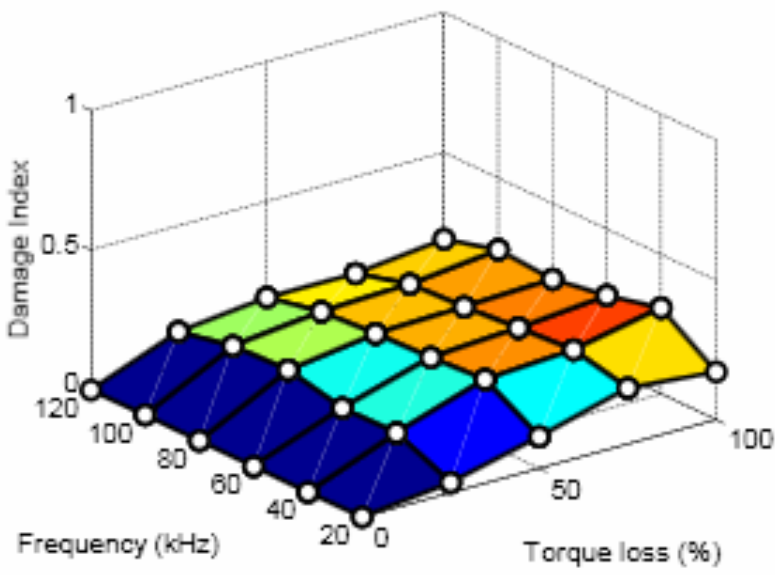

(c) $\mathrm{S} 4$ sensor

Figure 3-3-12. Damage index using the energy signals in time vs. B2 torque loss and excitation frequency, E2 excitation. 


\subsubsection{Bolt 2, Excitation Case IV}

In the final excitation configuration case (E4), sensing information is compiled at the three remaining sensors $(\mathrm{S} 1, \mathrm{~S} 2, \mathrm{~S} 3)$ located within the remaining three quadrants. In this scenario, the actuation occurred in the same composite panel (composite 2) where damage was present due to torque loss. Figure 3-3-13 displays the excitation and sensing configuration and indicates the Bolt 2 location of simulated damage. Figure 3-3-14 represents the scattering signal for a $40 \mathrm{kHz}$ excitation frequency under $\mathrm{E} 4$ actuation. The signal scattering at S1 and S2 sensors increase in magnitude as torque loss increases. The scattering signals at sensors across the joint from the source of actuation (S1, S2) are smaller in magnitude than the signal from the sensor that shares a composite panel with the source of actuation (S3).

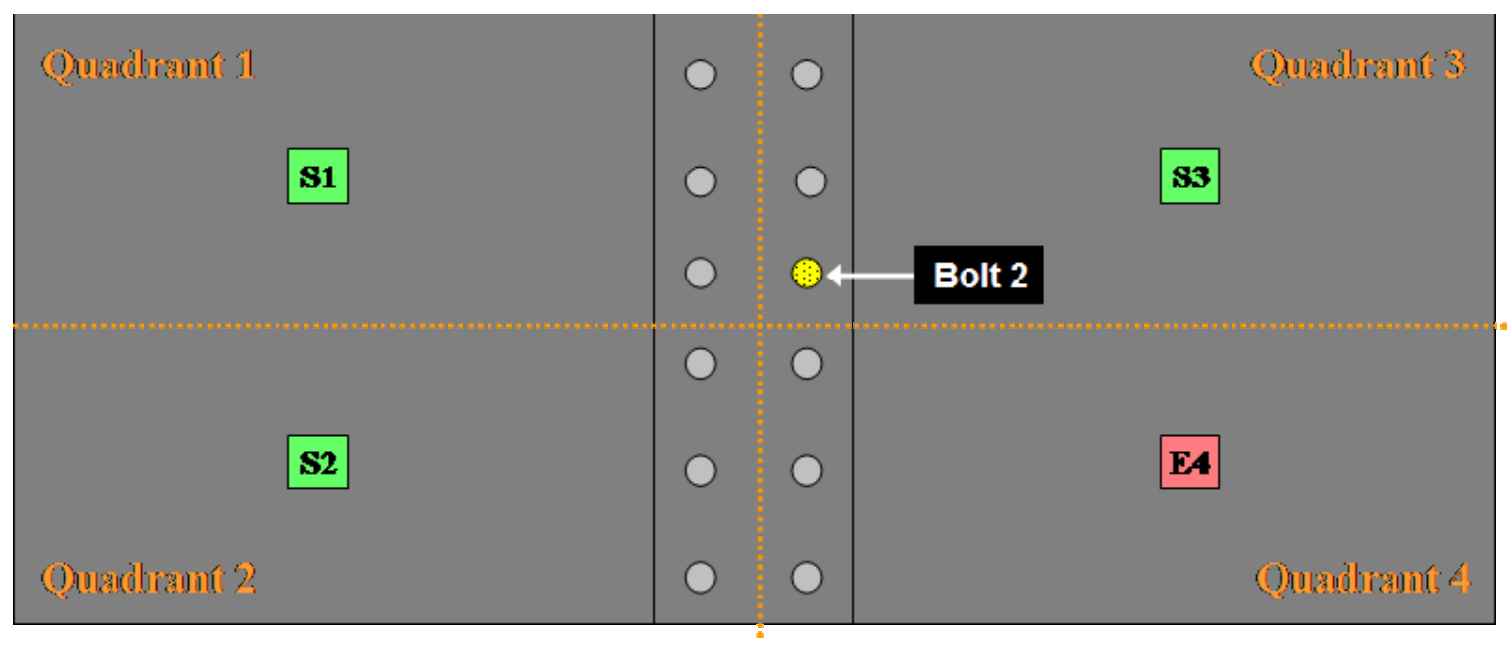

Figure 3-3-13. Excitation Case IV, variable Bolt 2 torque loss.

Figure 3-3-15 presents the damage index using the energy magnitudes due to frequency response $\left(D I_{f}\right)$ at $\mathrm{S} 1, \mathrm{~S} 2$ and S3 under E4 excitation. Figure 3-3-16 presents the damage index using the signals in time $\left(D I_{t}\right)$ at $\mathrm{S} 1, \mathrm{~S} 2$ and $\mathrm{S} 3$ sensing locations. 
Damage index due to time signal and due to frequency magnitude both exhibit a higher sensitivity to damage due to torque loss as compared to excitation frequency. Similar to Bolt 0 and Bolt 1, the damage detection capabilities using time and frequency response magnitudes are favorable within the jointed composite panel due to Bolt 2 torque loss even when actuation occurred in the same composite panel (composite 2) as damage due to torque loss.

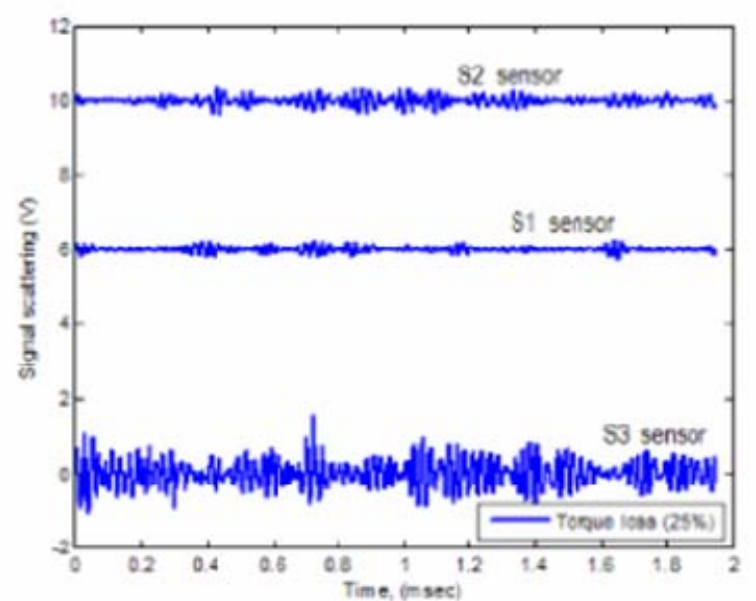

a) $25 \%$ torque loss

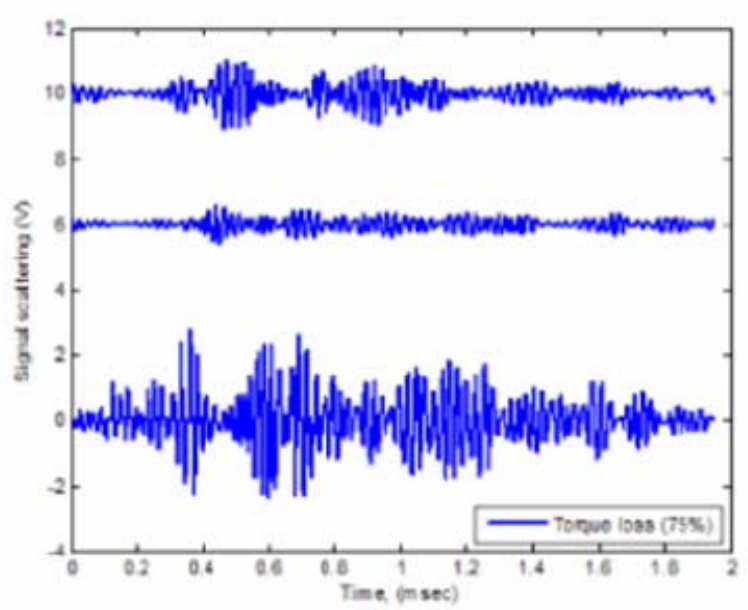

c) $75 \%$ torque loss

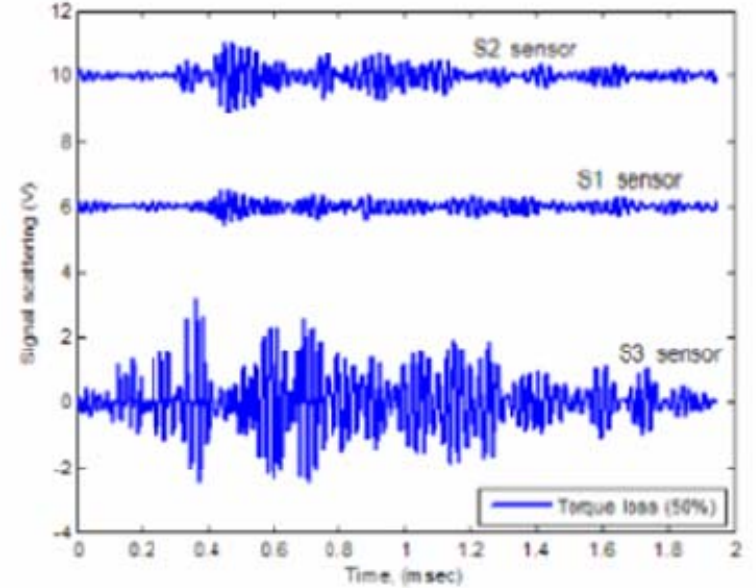

b) $50 \%$ torque loss

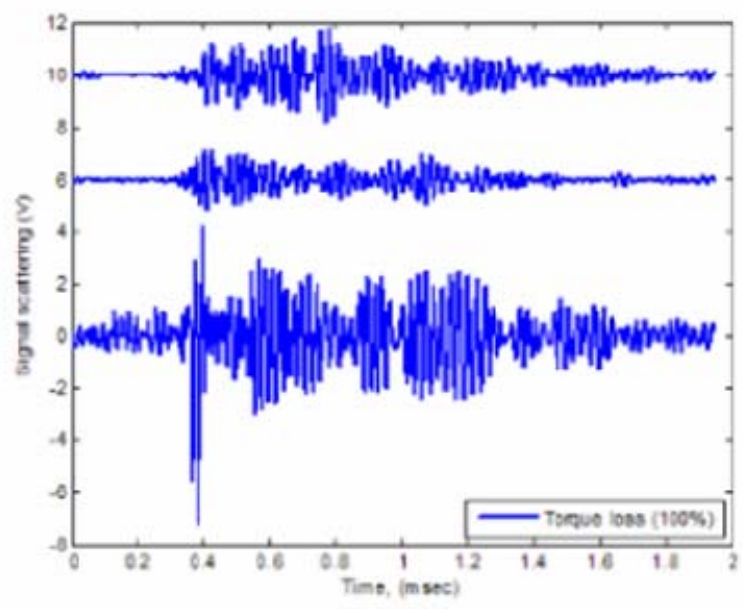

d) $100 \%$ torque loss

Figure 3-3-14. Scattering signals, B2 torque loss, E4 $40 \mathrm{kHz}$ excitation frequency. 


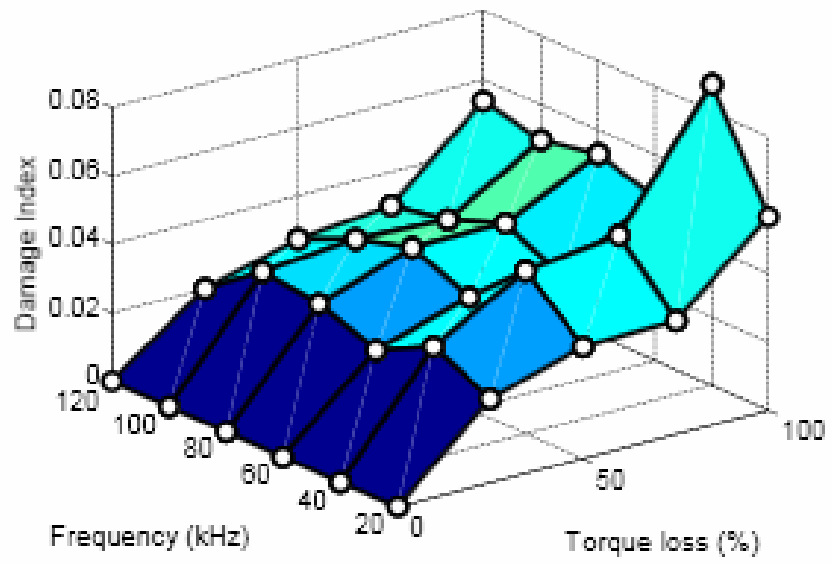

(a) $\mathrm{S} 1$ sensor

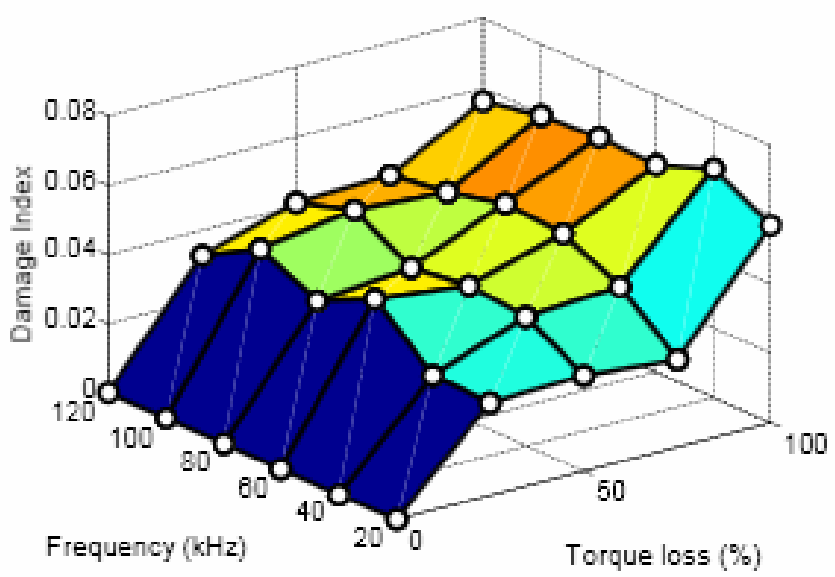

(b) $\mathrm{S} 2$ sensor

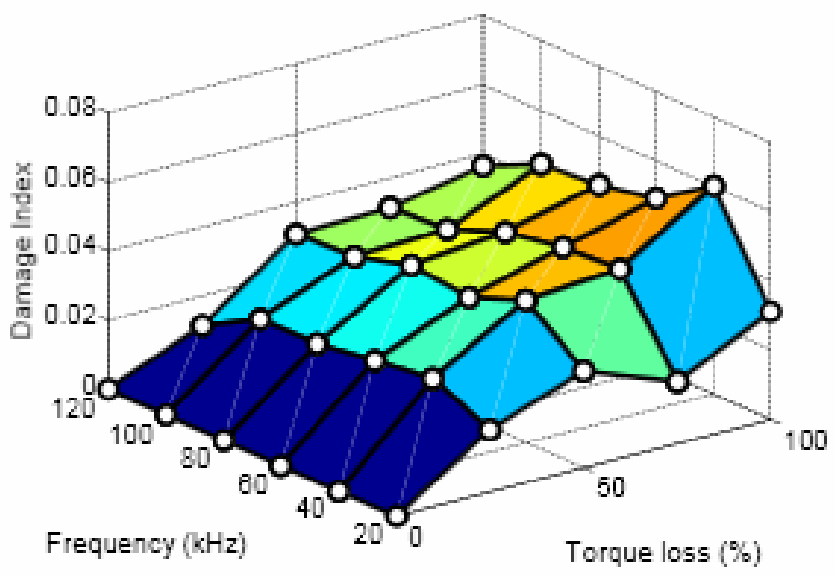

(c) $\mathrm{S} 3$ sensor

Figure 3-3-15. Damage index using the energy magnitudes in frequency vs. B2 torque loss and excitation frequency, E4 excitation. 


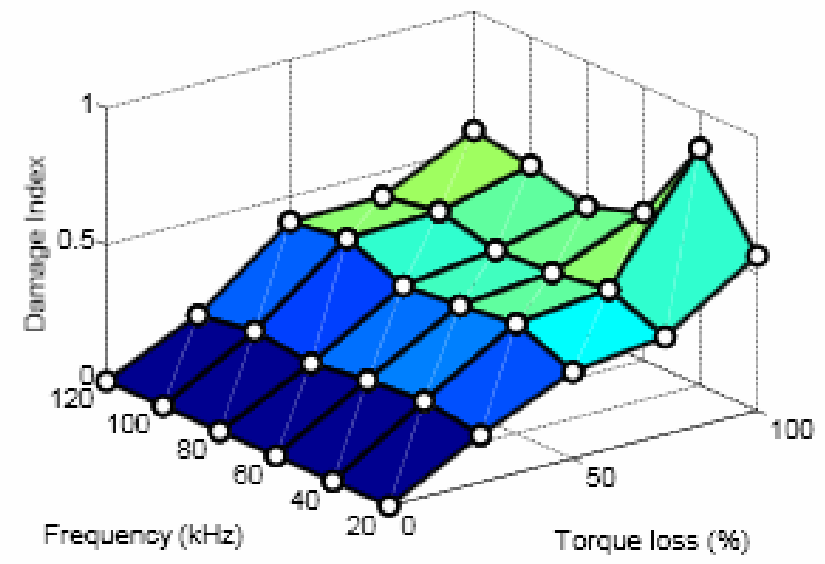

(a) $\mathrm{S} 1$ sensor

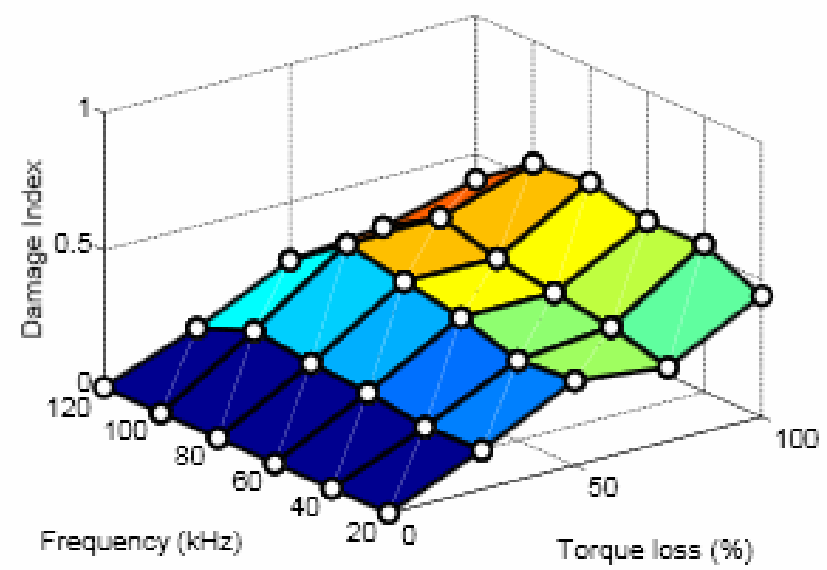

(b) $\mathrm{S} 2$ sensor

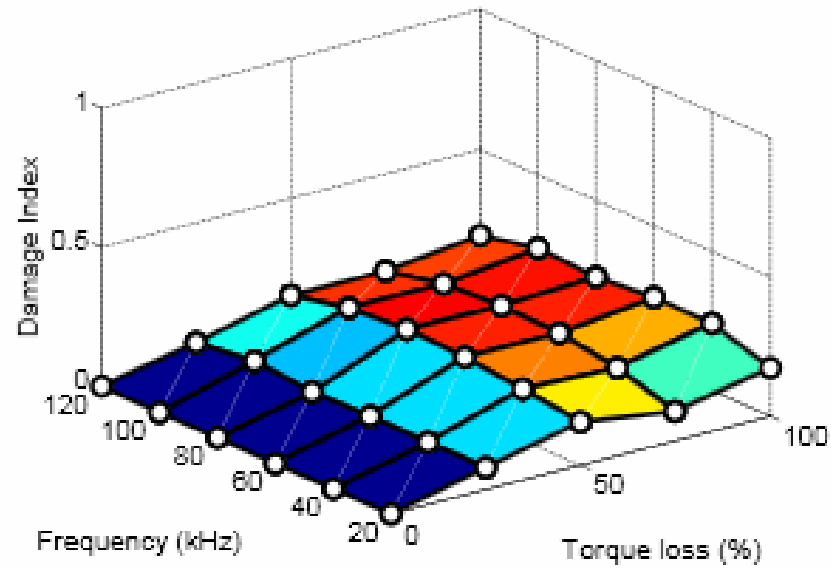

(c) $\mathrm{S} 3$ sensor

Figure 3-3-16. Damage index using the signals in time vs. B2 torque loss and excitation frequency, E4 excitation. 


\subsubsection{Comparison of Bolt 2 excitation configurations}

Figure 3-3-17 shows the side-by-side comparison of the normalized average damage index of the composite jointed panel due to Bolt 2 torque loss for each excitation configuration case (E1, E2, E3 and E4). Due to the proximity of Bolt 2 to Quadrant 4, the trends become less clear as compared to the trends from Bolt 0 and Bolt 1 . To provide a rough estimate of the location of damage due to torque loss within the jointed composite panel, the following analysis is made:

$$
\begin{array}{ll}
D I_{E 1, S 3} \approx D I_{E 1, S 4}>>D I_{E 1, S 2} & \text { Figure 3-3-17 (a) } \\
D I_{E 2, S 3}>D I_{E 2, S 4}>>D I_{E 2, S 1} & \text { Figure 3-3-17 (b) } \\
D I_{E 3, S 1} \approx D I_{E 3, S 2}>>D I_{E 3, S 4} & \text { Figure 3-3-17 (c) } \\
D I_{E 4, S 1} \approx D I_{E 4, S 2}>>D I_{E 4, S 3} & \text { Figure 3-3-17 (d) }
\end{array}
$$

While the difference between trends is less clear, the estimation still indicates damage present in-between sensors S1 and S3, thus within Quadrant 1 or Quadrant 3. The damage index quantities from S2 and S4 sensors have increased as compared to the information obtained from Bolt 0 and Bolt 1 torque loss (Figures 3-1-33 and 3-2-17). Therefore, the damage location can be identified toward the bottom of the area inbetween the S1 and S3 sensors. 


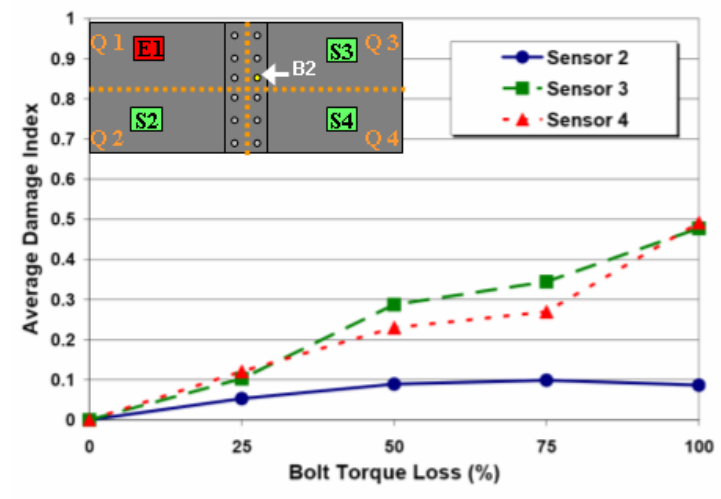

(a) E1 excitation

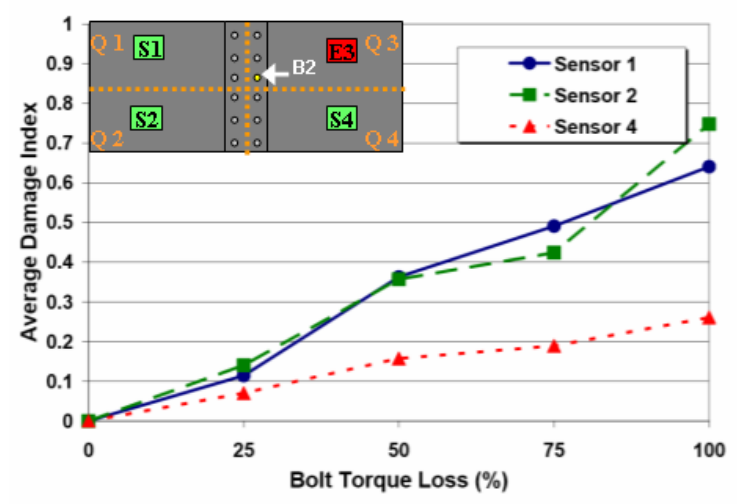

(c) E3 excitation

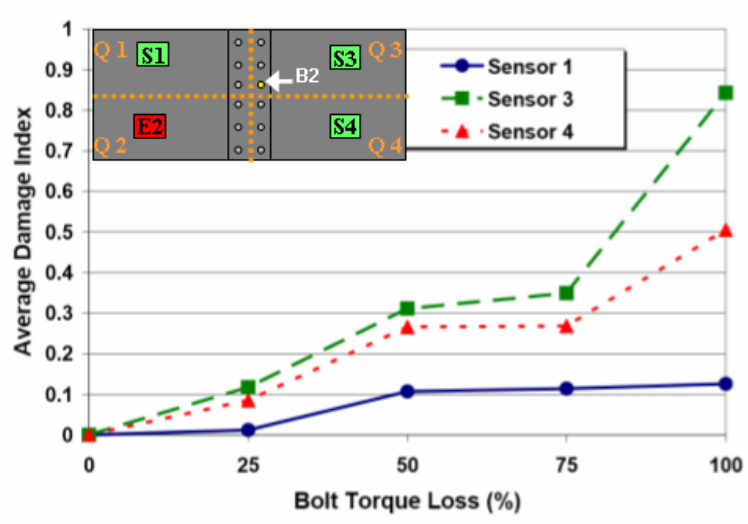

(b) E2 excitation

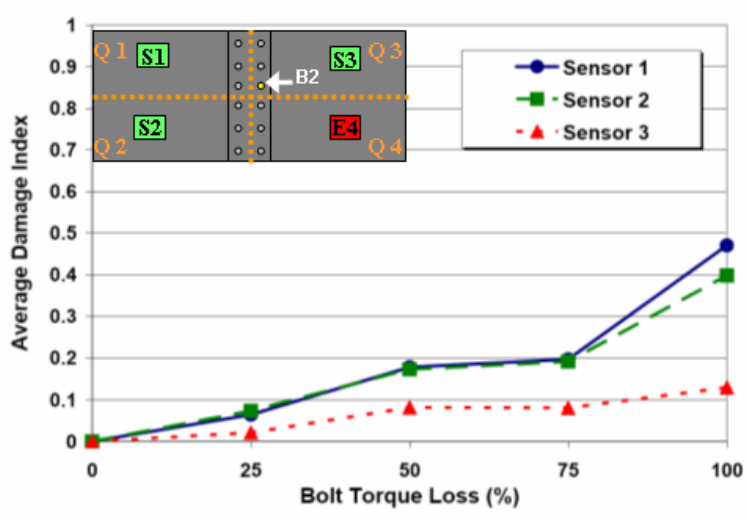

(d) E4 excitation

Figure 3-3-17. Comparison of normalized average damage index, B2 torque loss.

Similar to the analysis of Bolt 0 and Bolt 1 average damage index in sections 3.1.5 and 3.2.5, the clearest distinction of data for a possible damage location in-between sensors S1 and S3 comes from the E2 and E4 excitation cases. Due to Bolt 2 close proximity to Quadrant 4, the information obtained under excitation configuration E4 is not as clear when compared to information obtained under excitation configuration E3. However, at $100 \%$ torque loss, the distinction between S1 and S2 trends in Figure 3-3-17 (d) becomes clear. Again, this contributes to the idea that damage quantification plays a role in the discrimination between quadrants with possible damage. 
Figure 3-3-18 shows the graphical comparison of the average damage index values from $D I_{E 4, S 1}$ and $D I_{E 2, S 3}$ taken from Figure 3-3-17 (b) and Figure 3-3-17 (d).

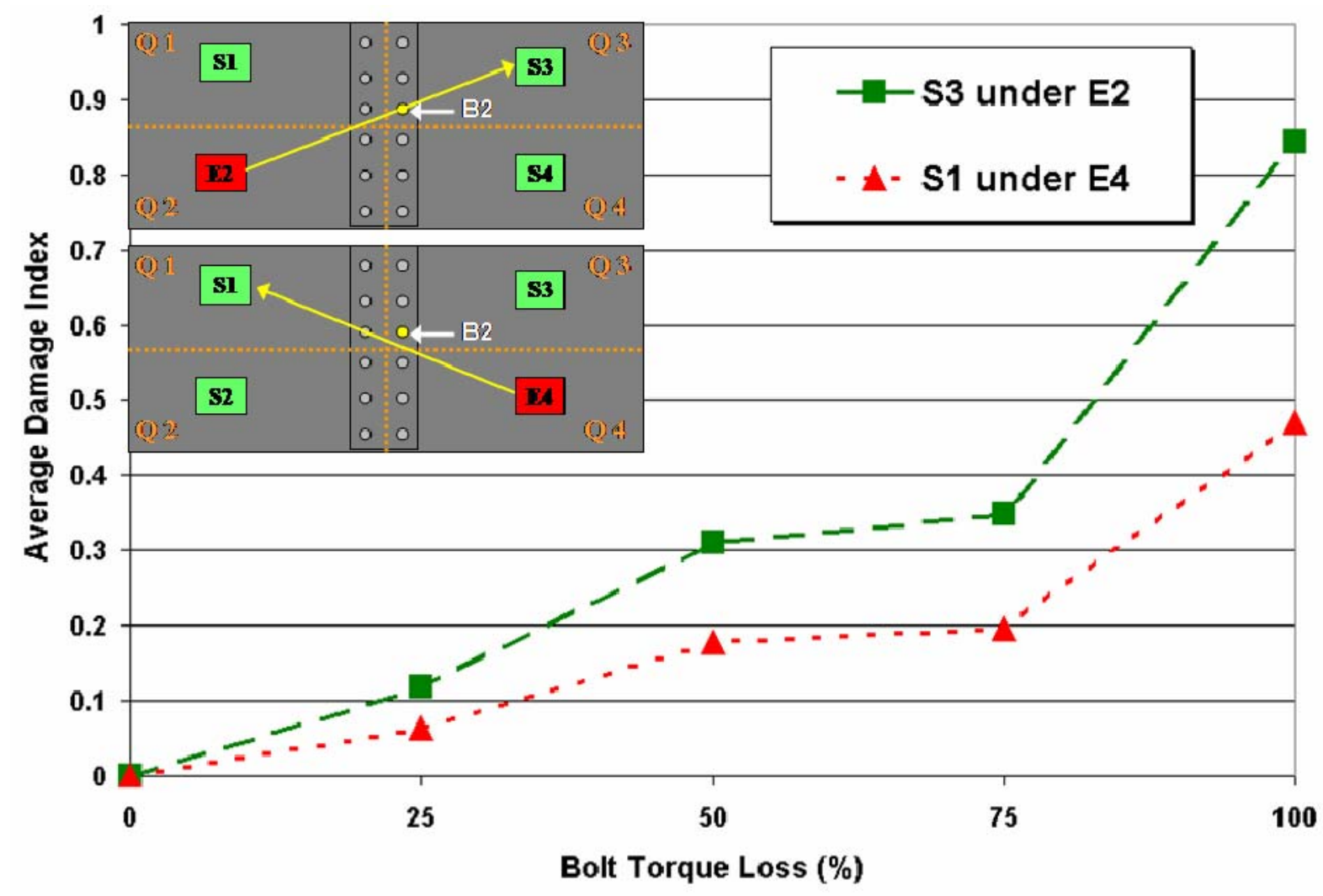

Figure 3-3-18. Comparison of normalized average damage indices, B2 torque loss; $D I_{E 2, S 3}$ and $D I_{E 4, S 1}$.

Comparing $D I_{E 4, S 1}$ and $D I_{E 2, S 3}$, Figure 3-3-18 clearly shows that for all torque loss values:

$$
D I_{E 2, S 3}>D I_{E 4, S 1}
$$

This trend indicates a higher damage index within proximity to S3, thus the greater likelihood of damage due to bolt torque loss in Quadrant 3 as compared to Quadrant 1, shown in Figure 3-3-19. 


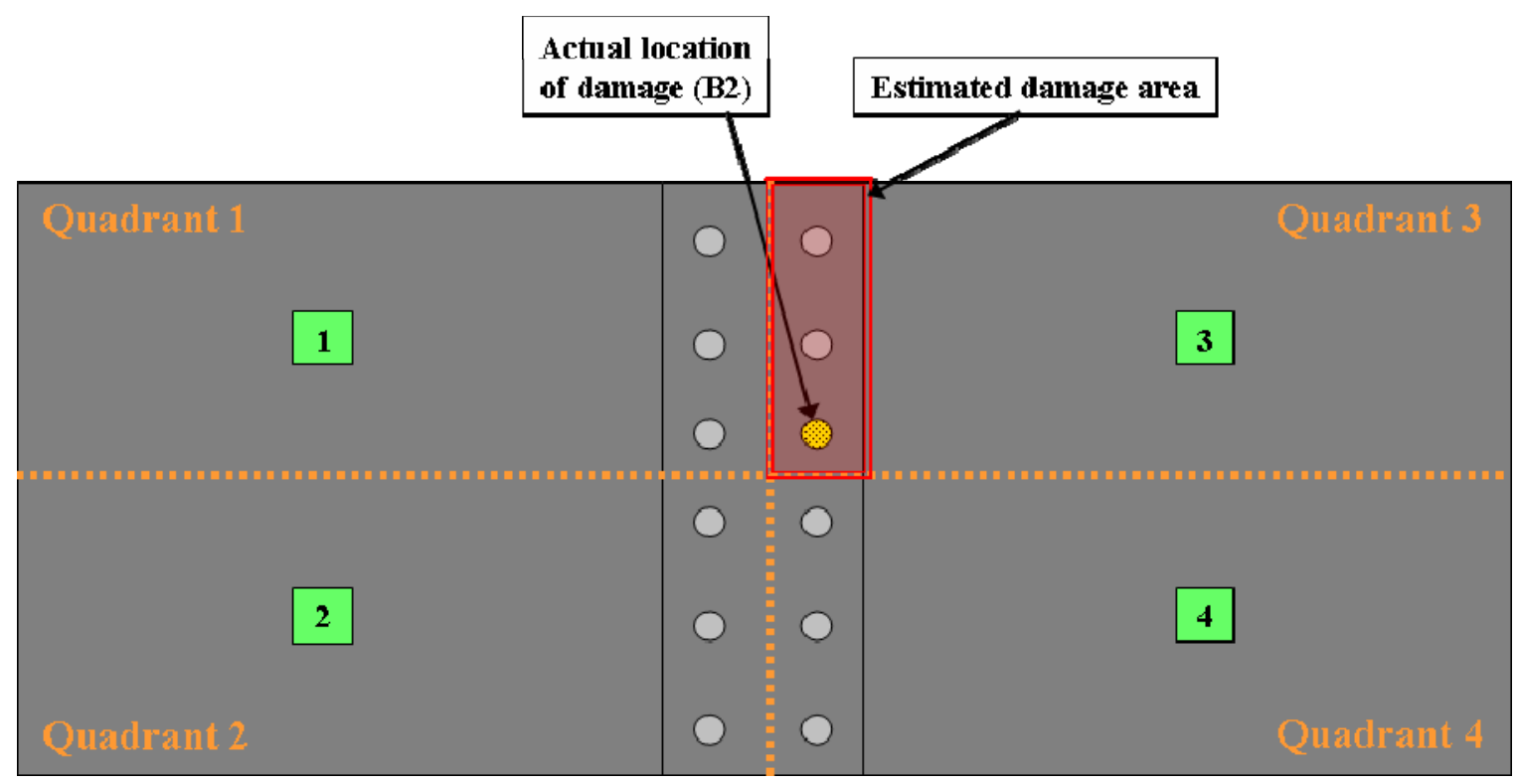

Figure 3-3-19. Estimated damage area due to B2 torque loss.

\subsection{Bolt 6 damage due to torque loss}

Similar to previous sections, the torque of Bolt 6 is varied to evaluate the damage detection capabilities of the Lamb wave method under a different damage scenario. In this case, the damaged bolt lies within Quadrant 2 and on composite 1; this is directly diagonal of the previous cases which were present within composite 2 in Quadrant 3. Five torque values were tested at each of the four excitation configuration cases. Experimental results were compiled exactly as the previous sections. Analysis of results due to torque loss within Quadrant 2 provided insight into the symmetry properties of the Lamb wave method as a damage detection device. 


\subsubsection{Bolt 6, Excitation Case I}

Figure 3-4-1 shows the excitation and sensing configuration and indicates the Bolt 6 location of damage due to torque loss. Figure 3-4-2 presents the damage index using the energy magnitudes due to frequency response $\left(D I_{f}\right)$ at $\mathrm{S} 2, \mathrm{~S} 3$ and $\mathrm{S} 4$ under E1 excitation. Figure 3-4-3 presents the damage index using the signals in time $\left(D I_{t}\right)$ at $\mathrm{S} 2, \mathrm{~S} 3$ and $\mathrm{S} 4$ sensing locations. Similarly to bolts in Quadrant 3, the damage index displays a higher sensitivity due to damage level as compared to the sensitivity due to excitation frequency. Similar to bolts in Quadrant 3, the damage detection capabilities using time and frequency response magnitudes are favorable within the jointed composite panel due to Bolt 6 torque loss even when actuation occurred in the same composite panel (composite 1) as damage due to torque loss.

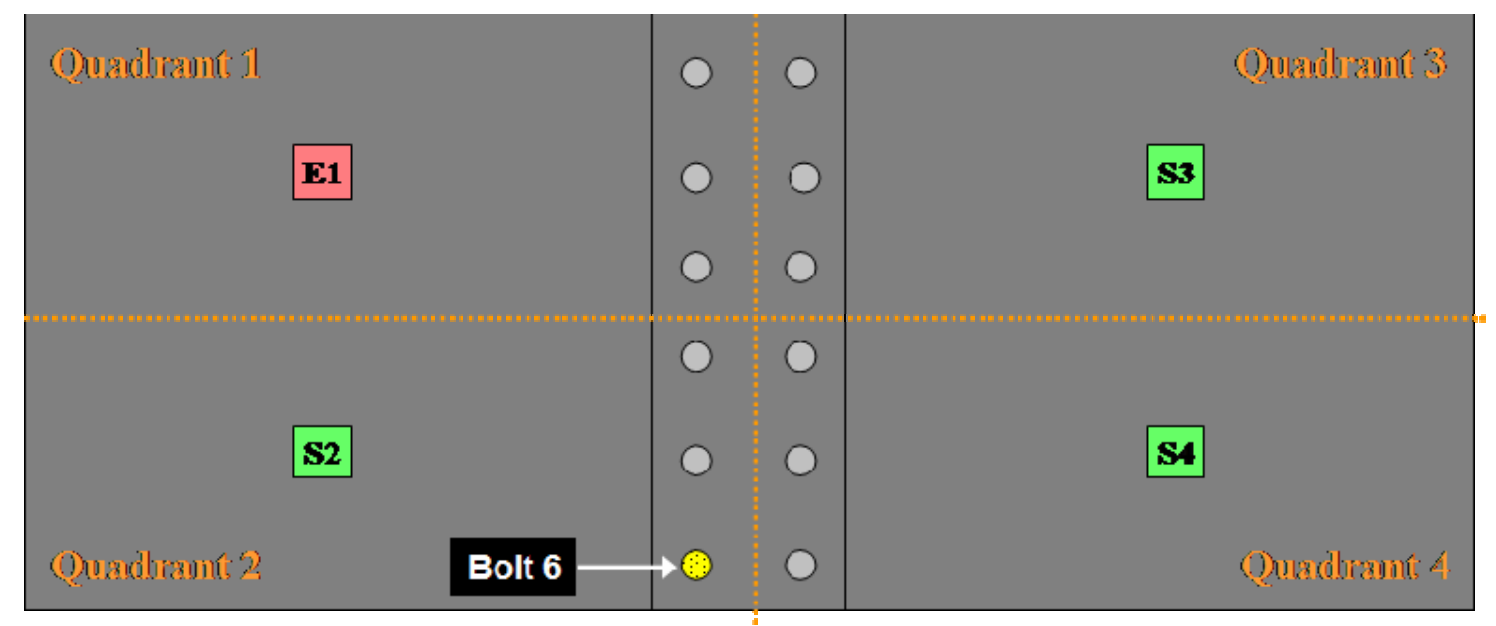

Figure 3-4-1. Excitation Case I, variable Bolt 6 torque loss. 


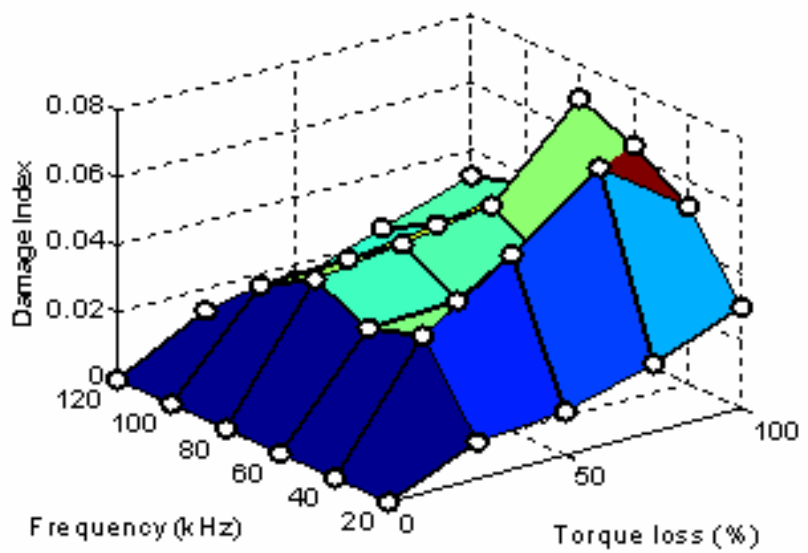

(a) $\mathrm{S} 2$ sensor

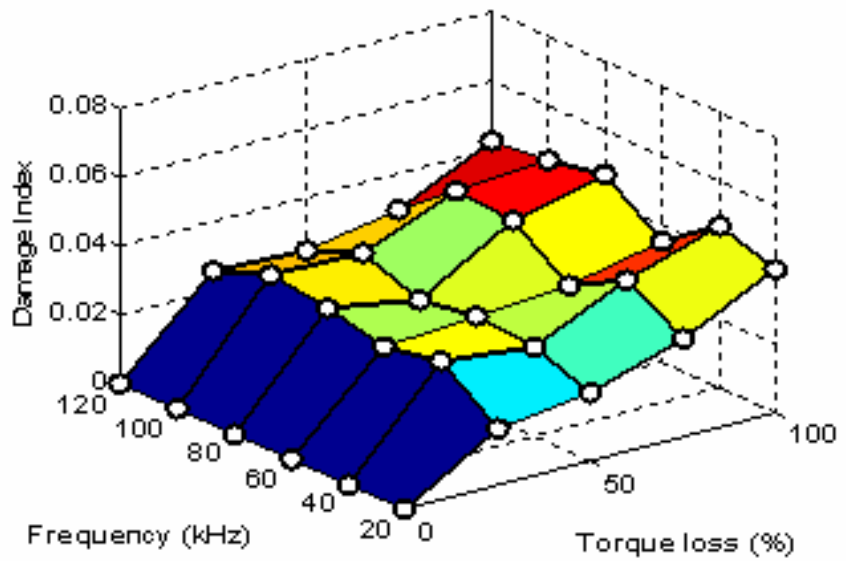

(b) S3 sensor

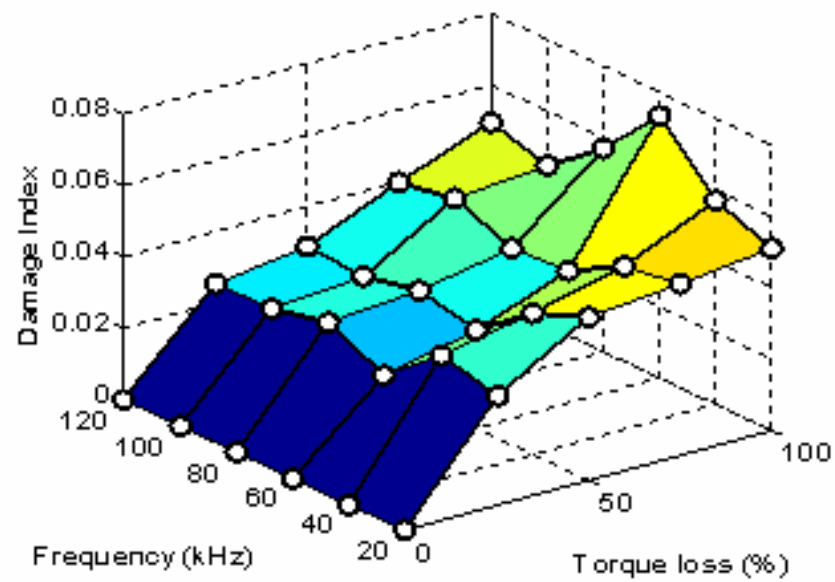

(c) S4 sensor

Figure 3-4-2. Damage index using the energy magnitudes in frequency vs. B6 torque loss and excitation frequency, E1 excitation. 


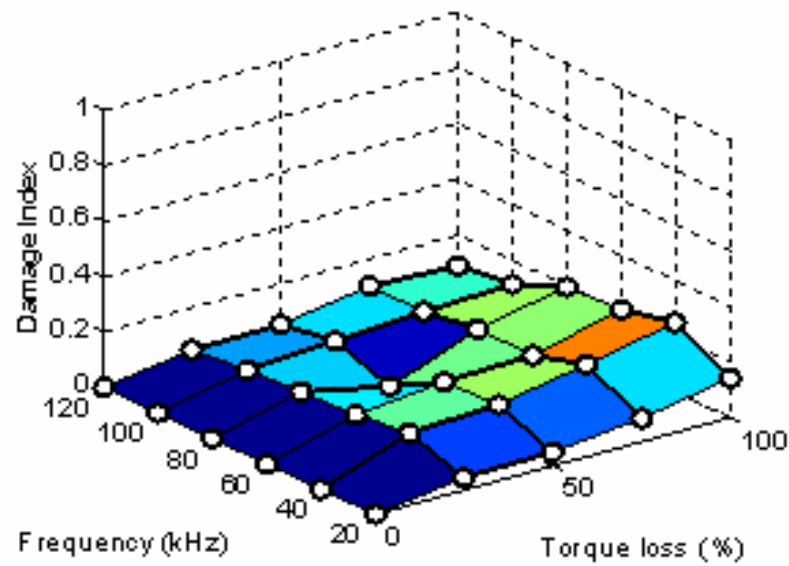

(a) S2 sensor

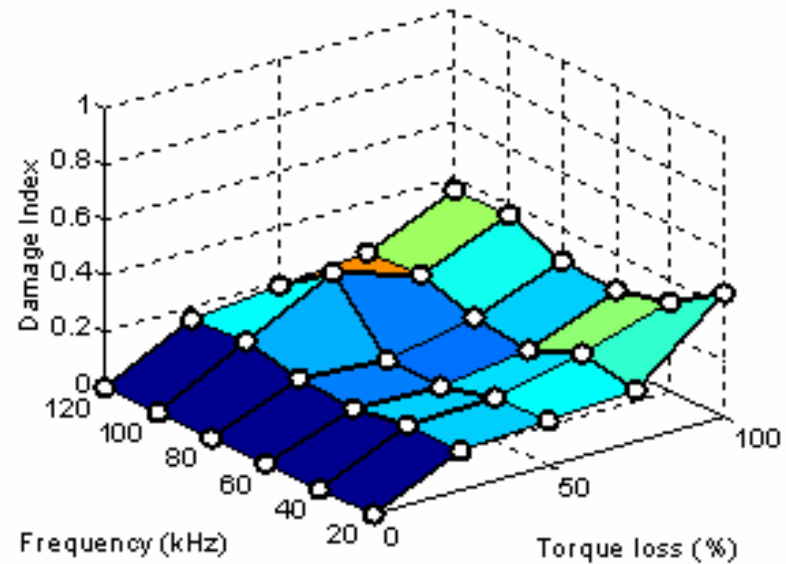

(b) S3 sensor

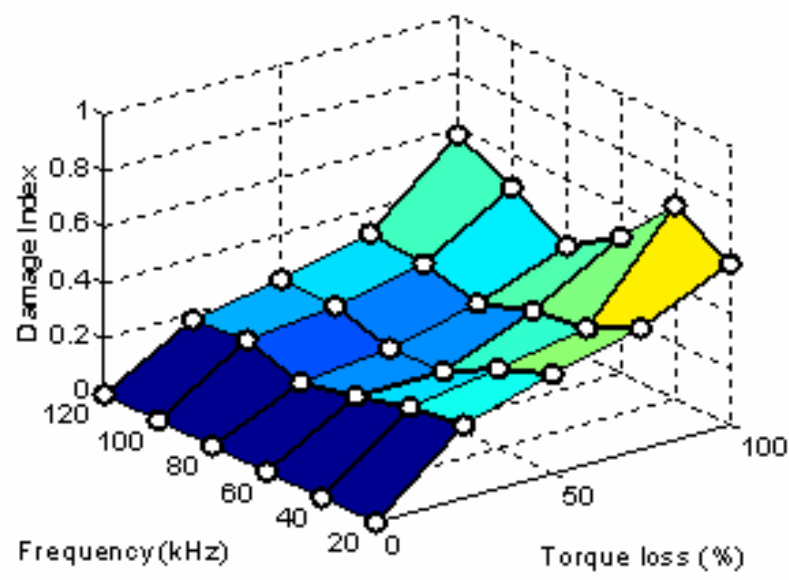

(c) $\mathrm{S} 4$ sensor

Figure 3-4-3. Damage index using the signals in time vs. B6 torque loss and excitation frequency, E1 excitation. 


\subsubsection{Bolt 6, Excitation Case II}

Using the piezo-patch in Quadrant 2 (E2) for excitation of the jointed composite panel, sensing information is compiled at the three remaining sensors (S1, S3, S4) located within the remaining three quadrants. In this excitation configuration, the actuation source was in the same quadrant as the damaged bolt. Figure 3-4-4 displays the excitation and sensing configuration and indicates the Bolt 6 location of simulated damage.

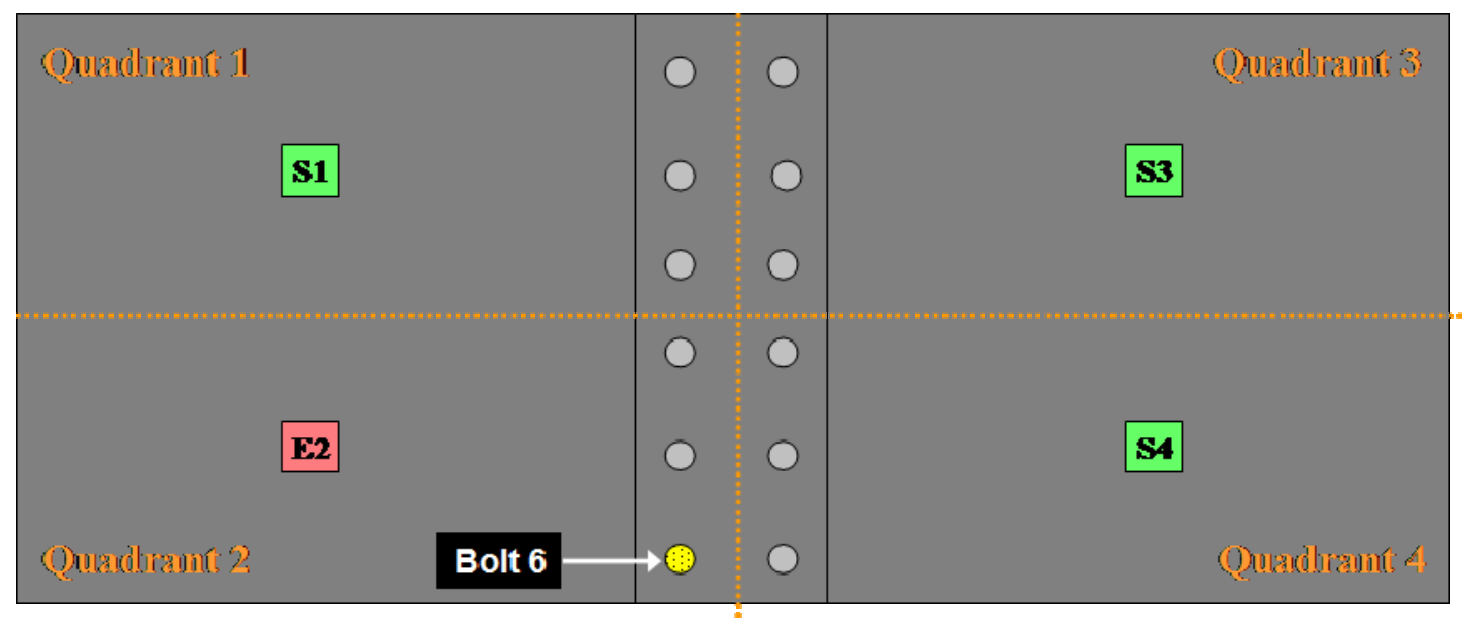

Figure 3-4-4. Excitation Case II, variable Bolt 6 torque loss.

Figure 3-4-5 presents the damage index using the energy magnitudes due to frequency response $\left(D I_{f}\right)$ at S1, S3 and S4 under E2 excitation. Figure 3-4-6 presents the damage index using the signals in time $\left(D I_{t}\right)$ at $\mathrm{S} 1, \mathrm{~S} 3$ and $\mathrm{S} 4$ sensing locations. Similar to bolts in Quadrant 3 under excitation configuration E3, the damage index displays the same sensitivity to bolt torque loss. 


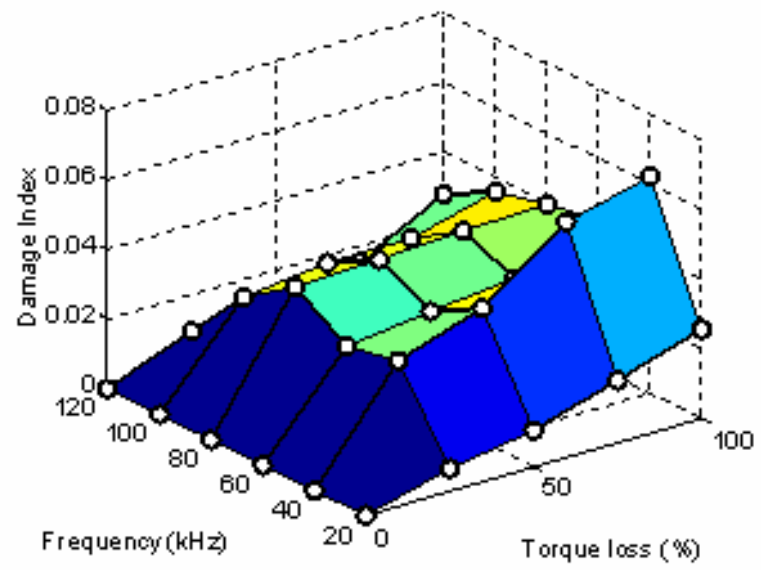

(a) S1 sensor

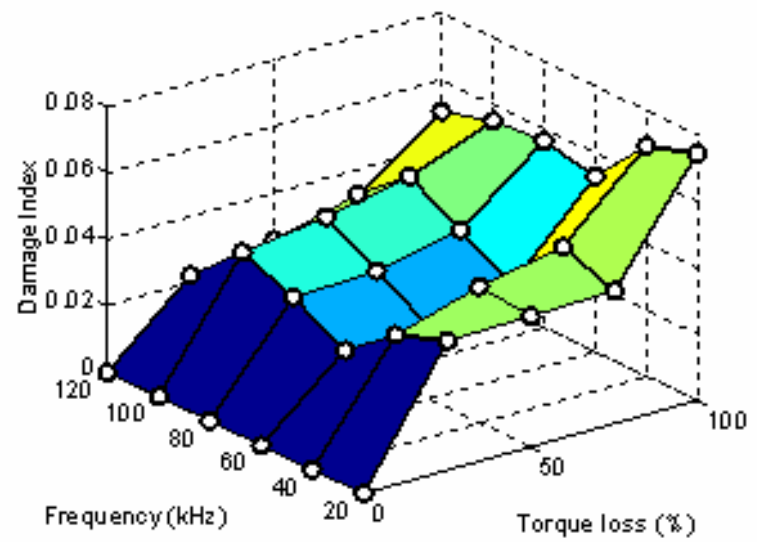

(b) S3 sensor

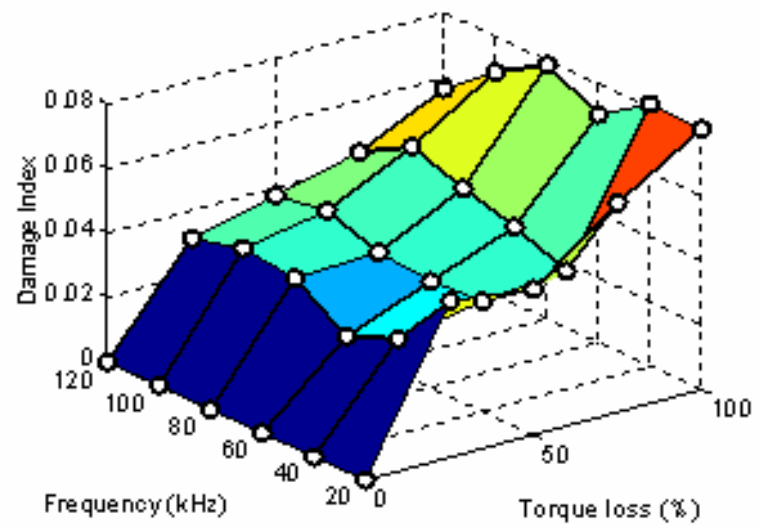

(c) S4 sensor

Figure 3-4-5. Damage index using the energy magnitudes in frequency vs. B6 torque loss and excitation frequency, E2 excitation. 


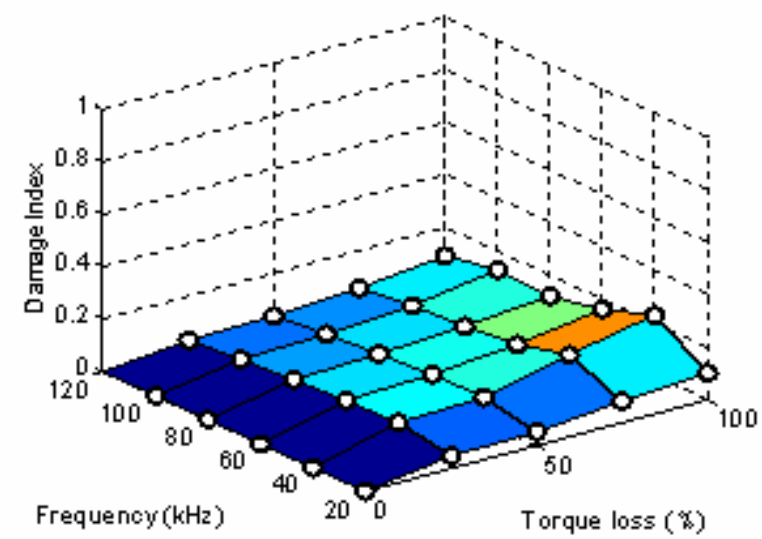

(a) S1 sensor

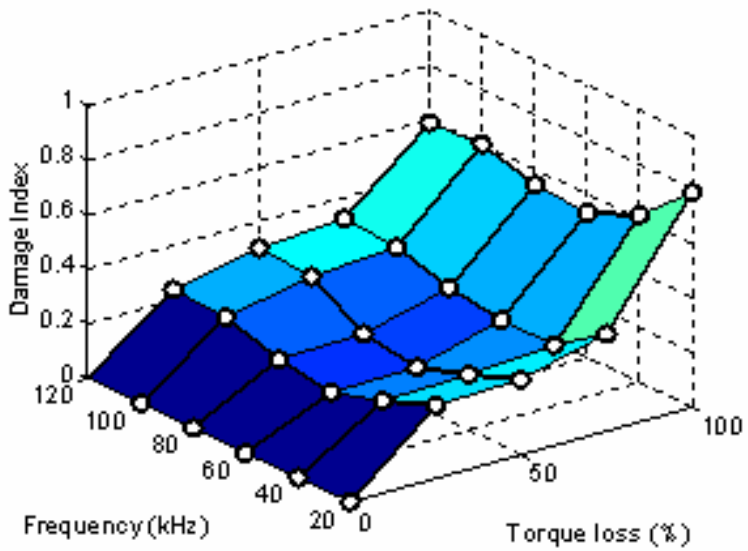

(b) S3 sensor

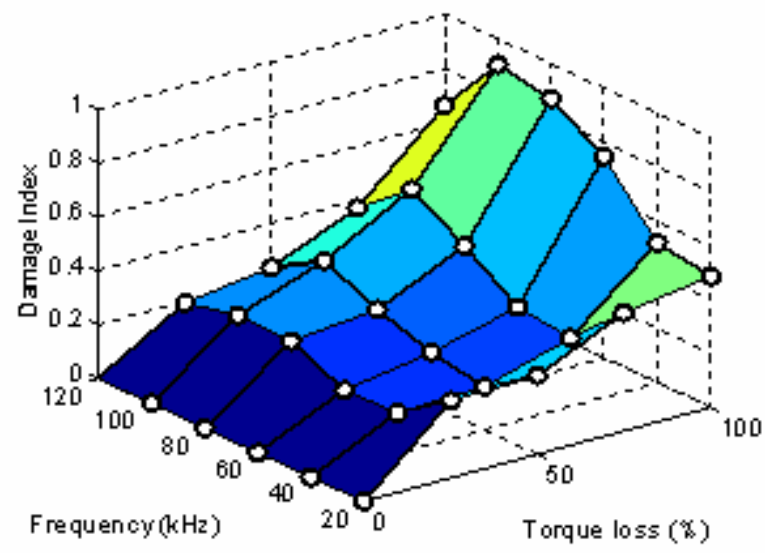

(c) $\mathrm{S} 4$ sensor

Figure 3-4-6. Damage index using the signals in time vs. B6 torque loss and excitation frequency, E2 excitation. 


\subsubsection{Bolt 6, Excitation Case III}

Figure 3-4-7 displays the excitation and sensing configuration and indicates the Bolt 6 location of simulated damage for the $\mathrm{E} 3$ excitation configuration case.

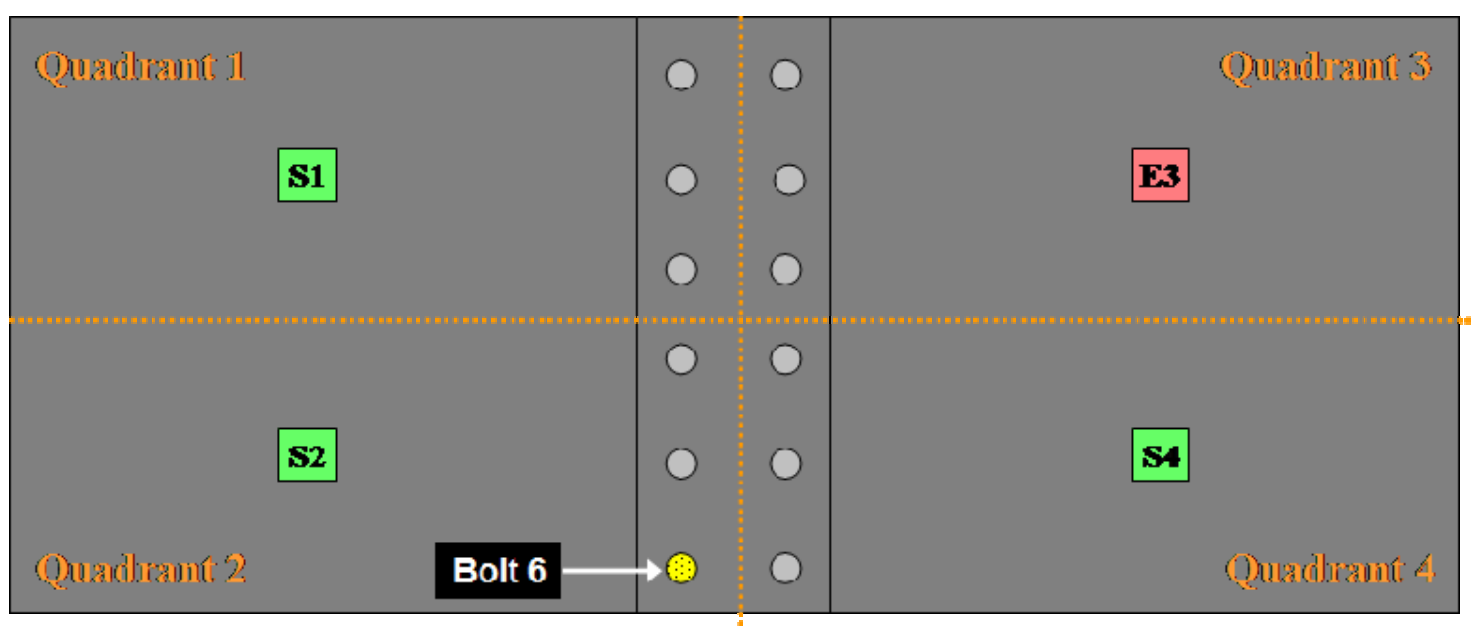

Figure 3-4-7. Excitation Case III, variable Bolt 6 torque loss.

Figure 3-4-8 presents the damage index using the energy magnitudes due to frequency response $\left(D I_{f}\right)$ at S1, S2 and S4 under E3 excitation. Figure 3-4-9 presents the damage index using the signals in time $\left(D I_{t}\right)$ at $\mathrm{S} 1, \mathrm{~S} 2$ and $\mathrm{S} 4$ sensing locations. The damage detection capabilities using time and frequency response magnitudes were favorable within the jointed composite panel due to Bolt 6 torque loss under the E3 excitation configuration. 


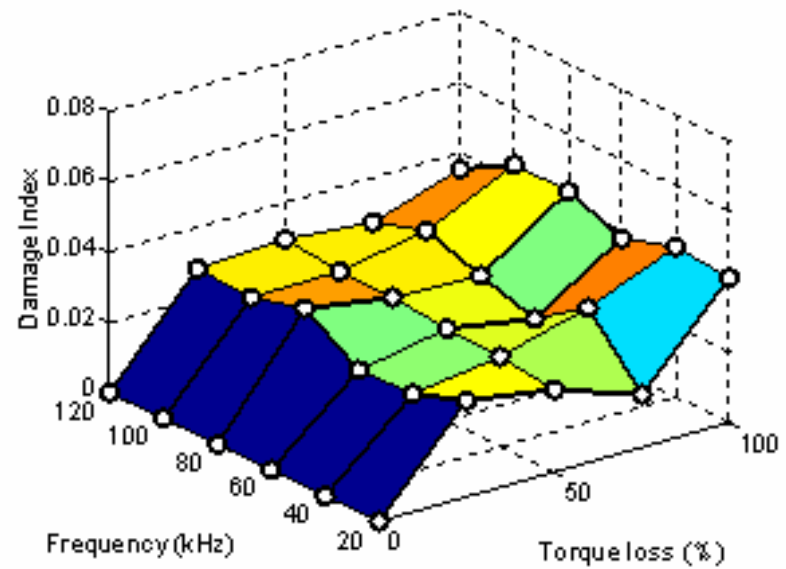

(a) S1 sensor

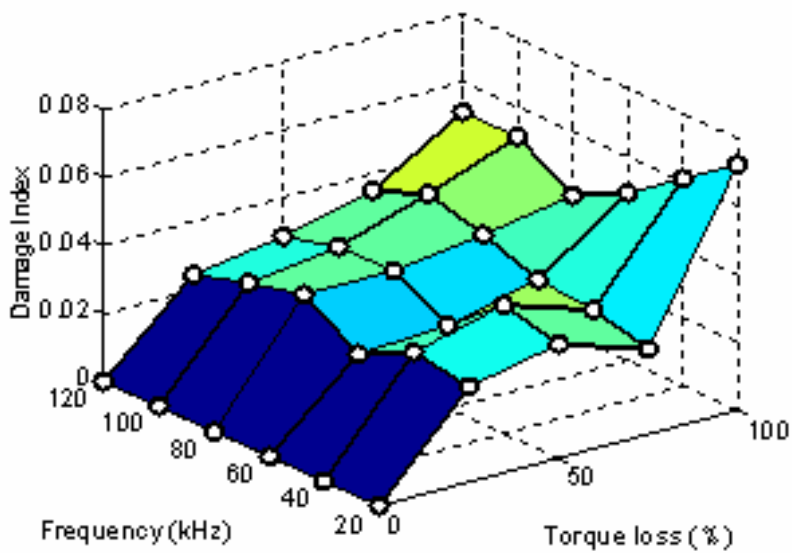

(b) S2 sensor

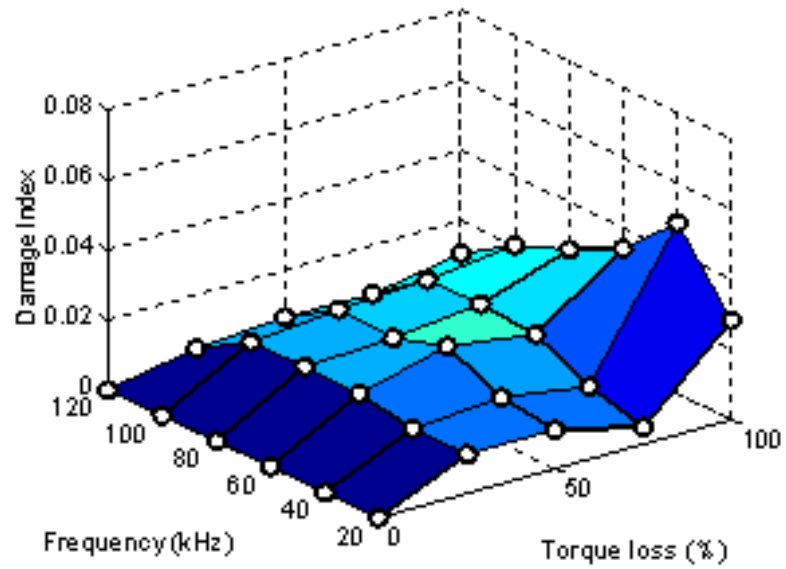

(c) S4 sensor

Figure 3-4-8. Damage index using the energy magnitudes in frequency vs. B6 torque loss and excitation frequency, E3 excitation. 


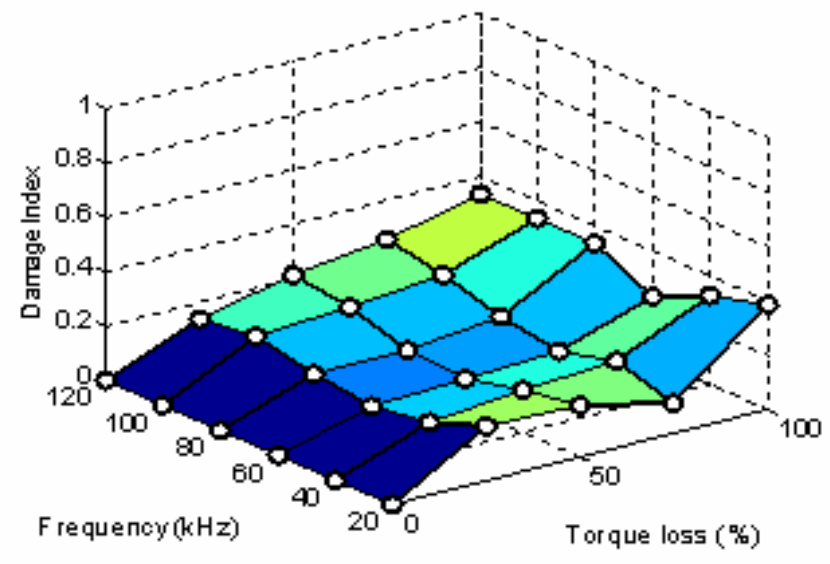

(a) S1 sensor

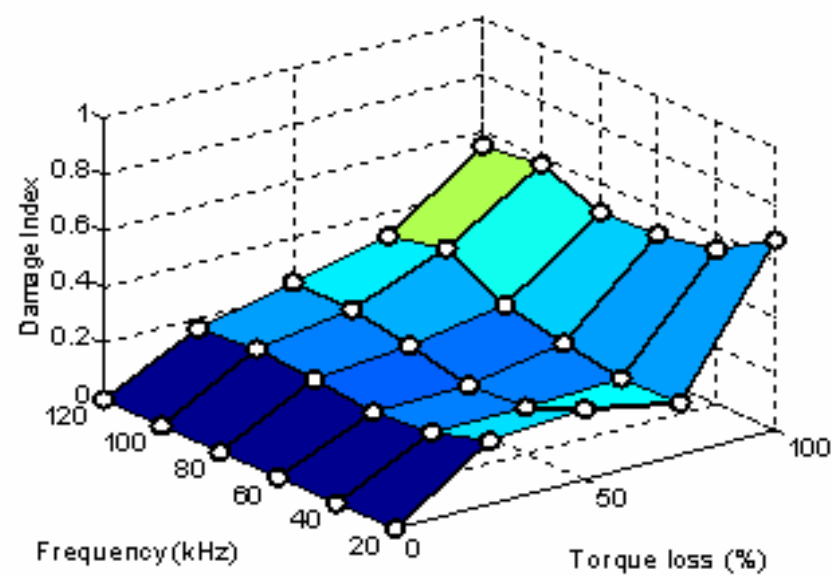

(b) $\mathrm{S} 2$ sensor

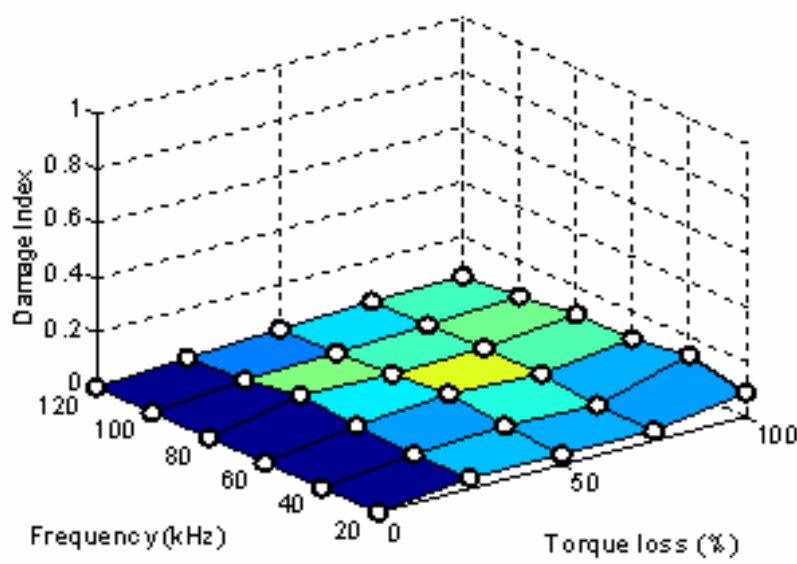

(c) S4 sensor

Figure 3-4-9. Damage index using the signals in time vs. B6 torque loss and excitation frequency, E3 excitation. 


\subsubsection{Bolt 6, Excitation Case IV}

Figure 3-4-10 displays the excitation and sensing configuration and indicates the Bolt 6 location of simulated damage for the E4 excitation configuration case.

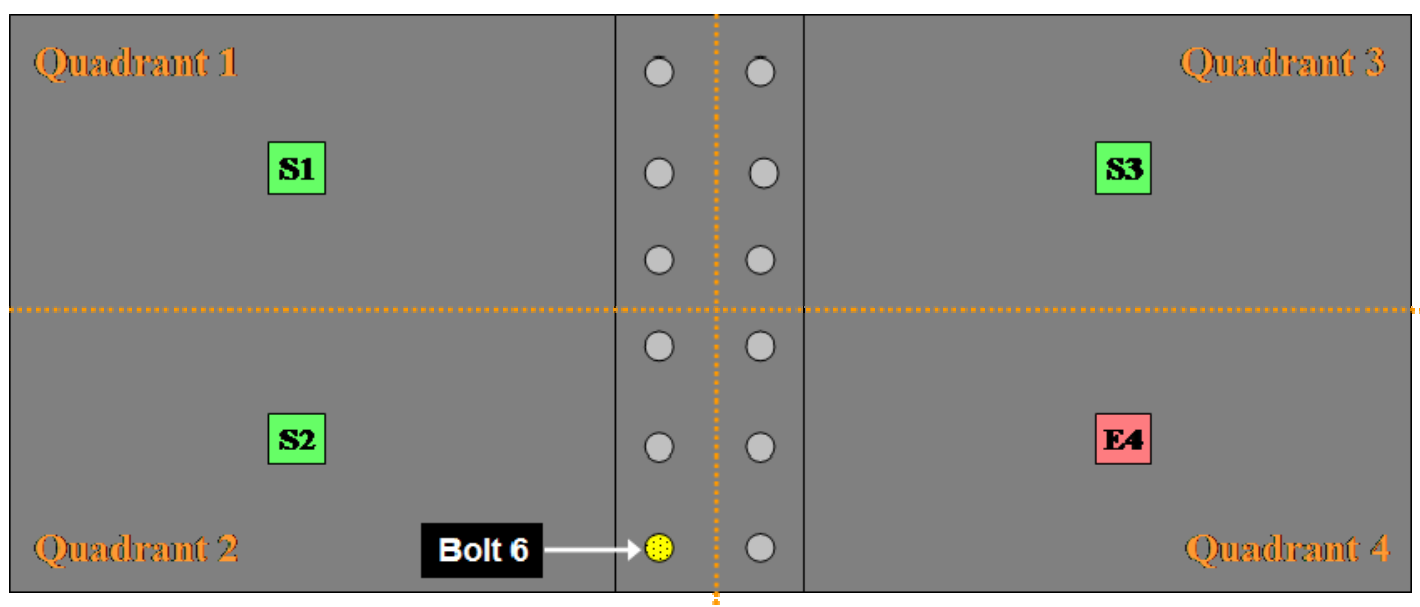

Figure 3-4-10. Excitation Case IV, variable Bolt 6 torque loss.

Figure 3-4-11 presents the damage index using the energy magnitudes due to frequency response $\left(D I_{f}\right)$ at $\mathrm{S} 1, \mathrm{~S} 2$ and S3 under E4 excitation. Figure 3-4-12 presents the damage index using the signals in time $\left(D I_{t}\right)$ at $\mathrm{S} 1, \mathrm{~S} 2$ and $\mathrm{S} 3$ sensing locations. The damage detection capabilities using time and frequency response magnitudes were favorable within the jointed composite panel due to Bolt 6 torque loss under the E4 excitation configuration. 


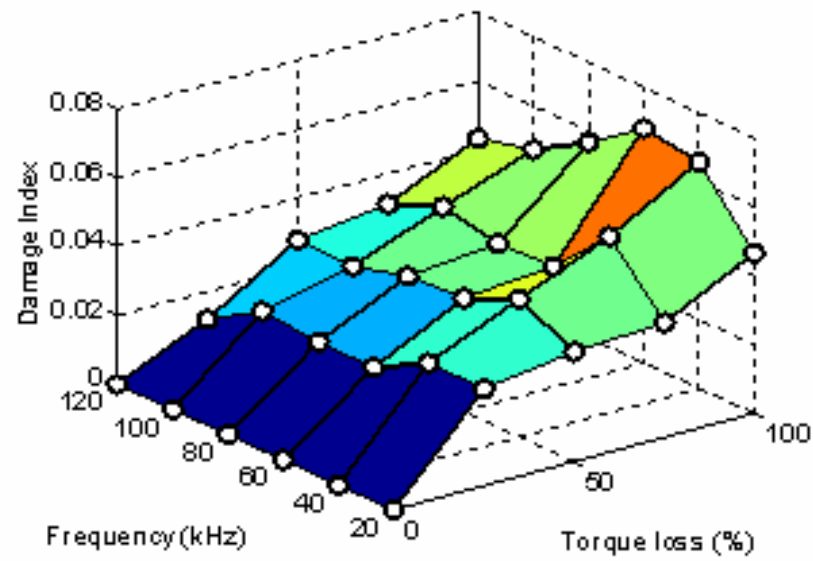

(a) S1 sensor

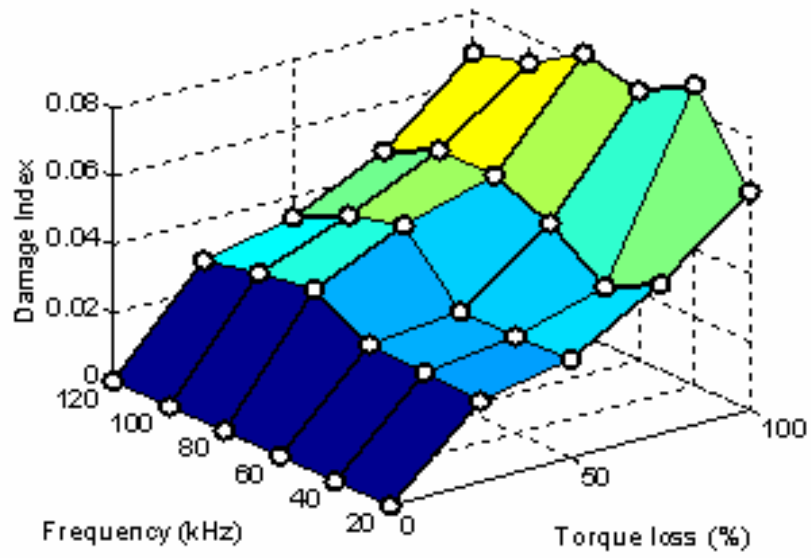

(b) S2 sensor

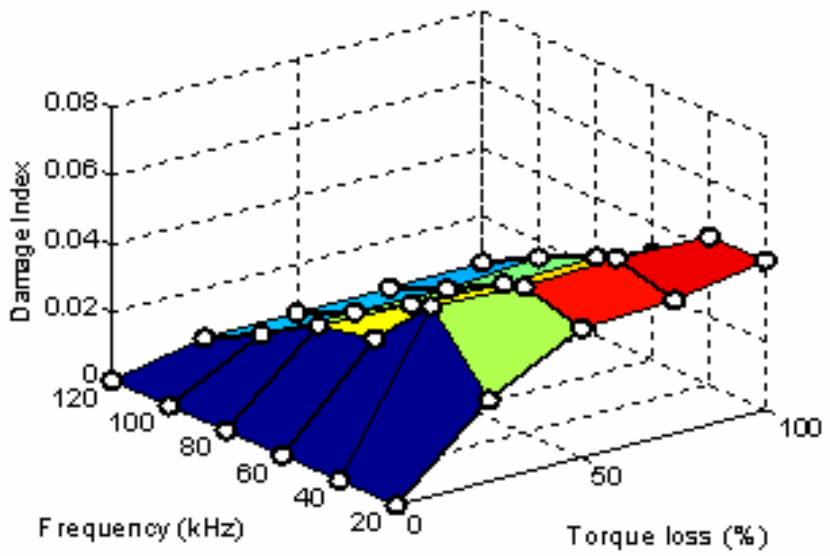

(c) S3 sensor

Figure 3-4-11. Damage index using the energy magnitudes in frequency vs. B6 torque loss and excitation frequency, E4 excitation. 


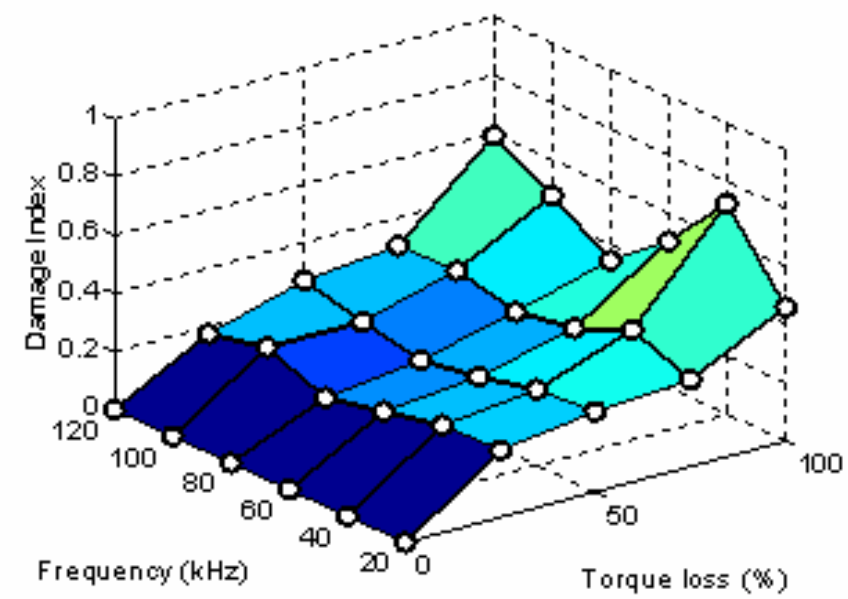

(a) S1 sensor

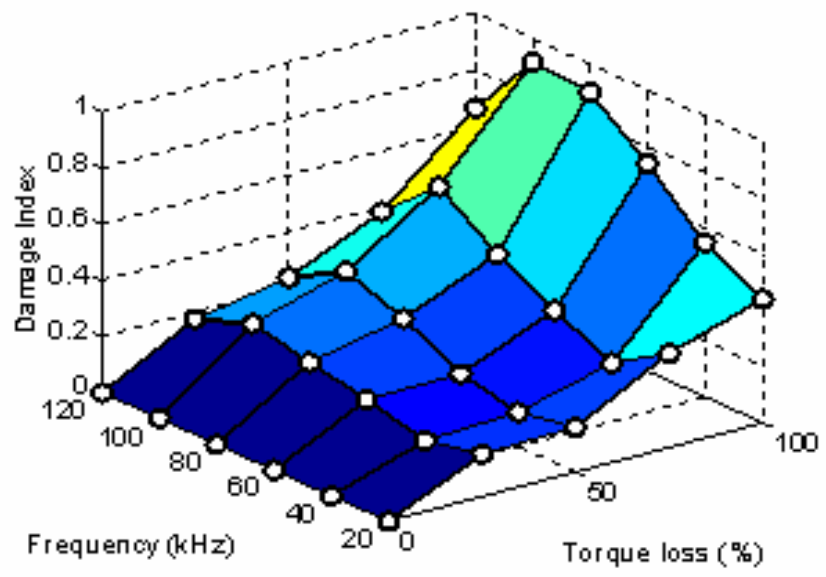

(b) $\mathrm{S} 2$ sensor

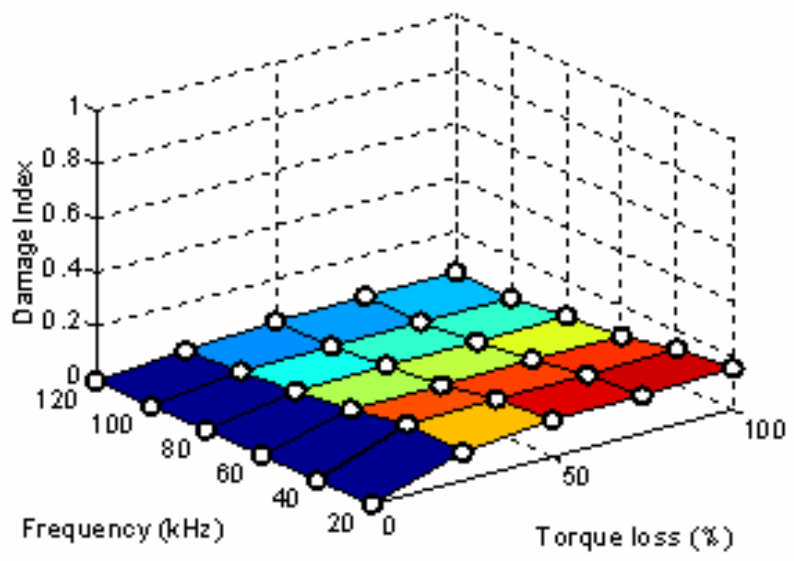

(c) S3 sensor

Figure 3-4-12. Damage index using the signals in time vs. B6 torque loss and excitation frequency, E4 excitation. 


\subsubsection{Comparison of Bolt 6 excitation configurations}

Figure 3-4-13 shows the comparison of the normalized average damage index of the composite jointed panel due to Bolt 6 torque loss for each excitation configuration case (E1, E2, E3 and E4). The sensitivity to damage was favorable in all excitation cases, as indicated by the average damage index quantities at all values of torque loss. While Bolt 6 is located in a different quadrant from Bolts 0,1 and 2, the average damage index trends remain the same, although in a symmetrically opposite manner. The following analysis was made to provide a rough estimate of the location of damage due to torque loss using the Lamb wave method:

$$
\begin{array}{rlrl}
D I_{E 1, S 4} & >D I_{E 1, S 3}>>D I_{E 1, S 2} & & \text { Figure 3-4-13 (a) } \\
D I_{E 2, S 4} \geq D I_{E 2, S 3}>>D I_{E 2, S 1} & & \text { Figure 3-4-13 (b) } \\
D I_{E 3, S 2}>D I_{E 3, S 1}>>D I_{E 3, S 4} & \text { Figure 3-4-13 (c) } \\
D I_{E 4, S 2} \geq D I_{E 4, S 1}>>D I_{E 4, S 3} & \text { Figure 3-4-13 (d) }
\end{array}
$$

Using these relationships, the estimation damage location lies in-between sensors S2 and S4, thus within Quadrant 2 or Quadrant 4. There was a clearer distinction between average $D I$ trends in Figure 3-4-13 (a) and Figure 3-4-13 (c); the information under E1 and E3 excitation configurations yielded more distinguishable results. This confirms the symmetry properties of the Lamb wave method under the experimental damage scenario, as the trends between Quadrant 2 and Quadrant 3 are directly opposite. This provides insight into the prediction of other bolt torque loss scenarios within Quadrant 2, namely Bolt 7 and Bolt 8. The average $D I$ values in Figure 3-4-13 (b) and Figure 3-4-13 (d) are 
less clear at torque loss values less than $75 \%$. It was determined that, similar to the results from Bolts 0,1 , and 2, when actuation exists within the quadrants of possible damage, the average damage index trends can not provide great insight toward precise damage localization.

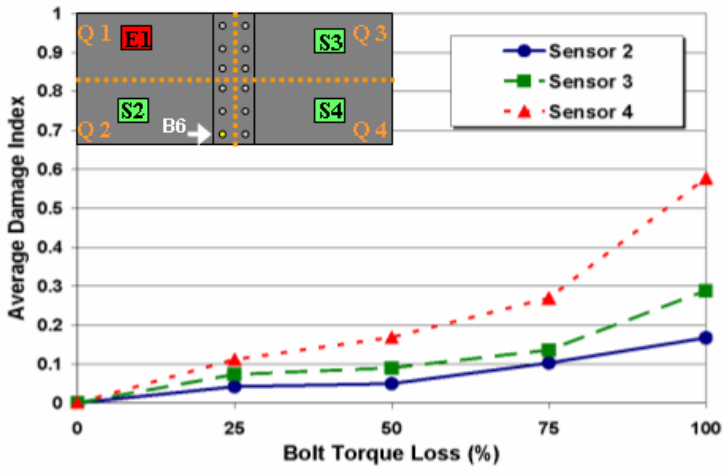

(a) E1 excitation

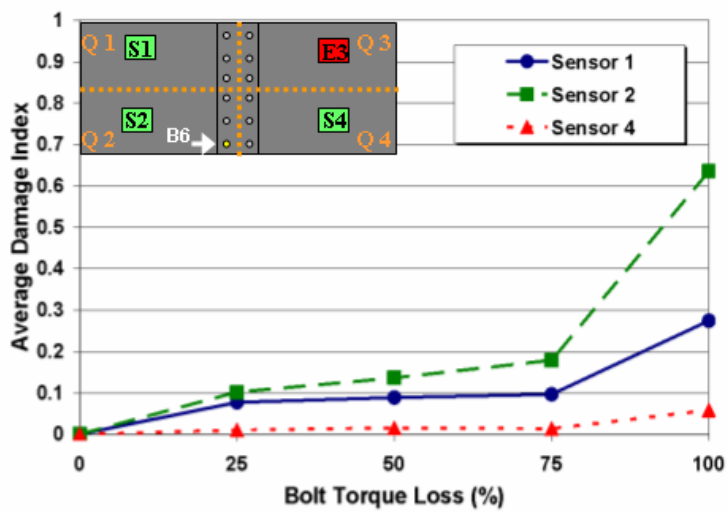

(c) E3 excitation

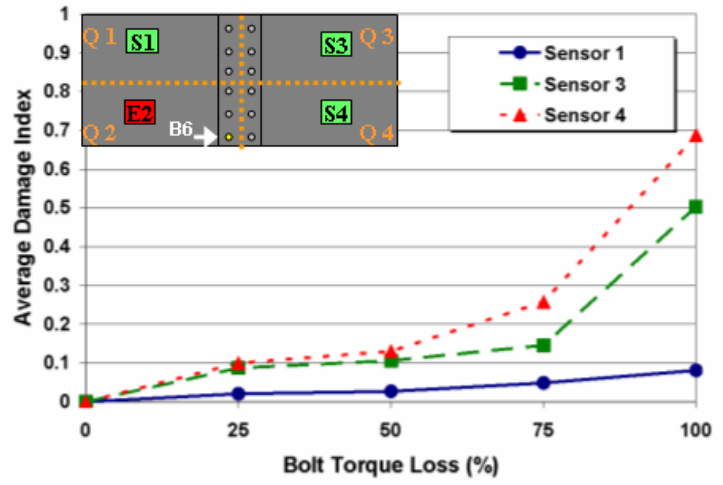

(b) E2 excitation

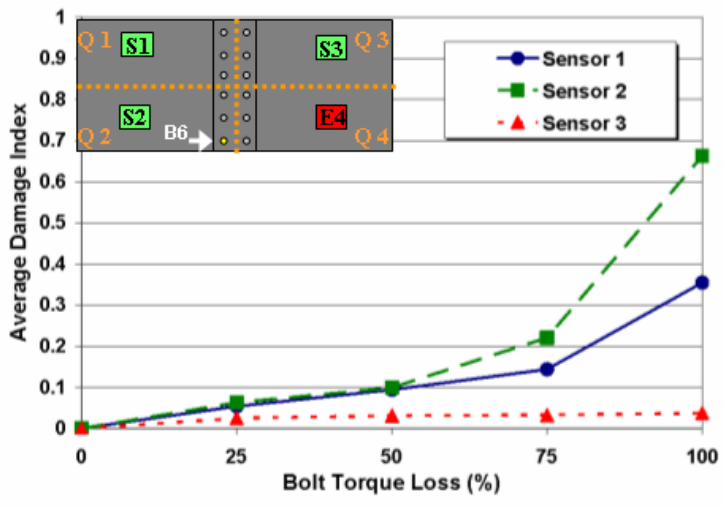

(d) E4 excitation

Figure 3-4-13. Comparison of normalized average damage index, B6 torque loss.

A clear distinction of data for possible damage location in-between sensors S2 and S4 comes from the E1 and E3 excitation cases. Figure 3-4-14 shows the comparison of $D I_{E 3, S 2}$ and $D I_{E 1, S 4}$ from Figure 3-4-13 (a) and Figure 3-4-13 (c). 


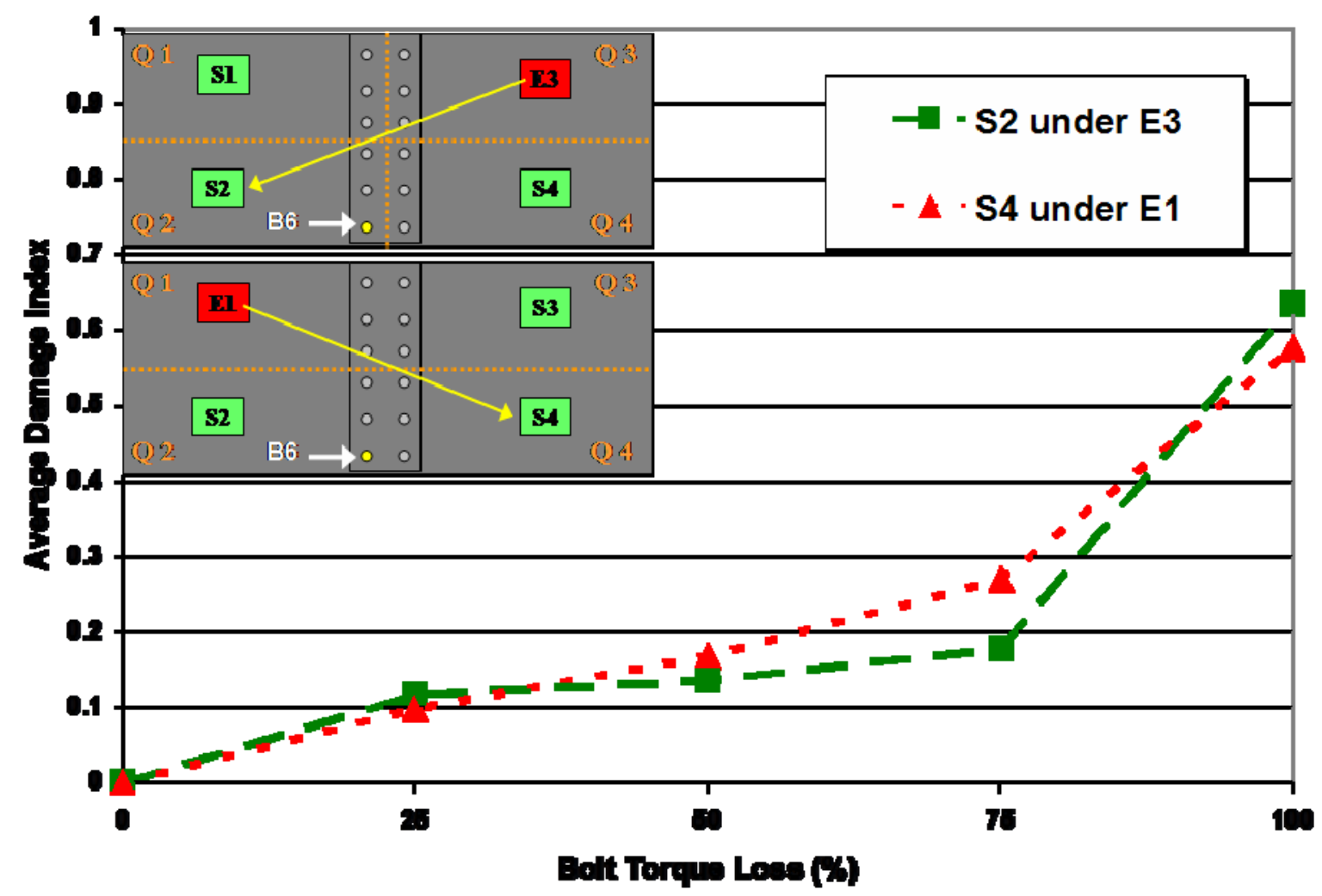

Figure 3-4-14. Comparison of normalized average damage indices, B6 torque loss; $D I_{E 3, S 2}$ and $D I_{E 1, S 4}$.

Figure 3-4-14 clearly shows that for all torque loss values of $25 \%$ and $100 \%$ :

$$
D I_{E 3, S 2}>D I_{E 1, S 4}
$$

This trend indicates a higher damage index within proximity to S2, thus the greater likelihood of damage due to bolt torque loss in Quadrant 2 as compared to Quadrant 4. However, at Bolt 6 torque loss values of $50 \%$ and $75 \%$ :

$$
D I_{E 3, S 2}<D I_{E 1, S 4}
$$


This would indicate the opposite to be true, that Quadrant 4 hosts a greater likelihood of damage due to torque loss. To determine which of these statements provides the accurate insight, another damage index trend comparison was made, this time utilizing the samecomposite actuation-sensing information. Figure 3-4-15 shows the comparison of $D I_{E 1, S 2}$ and $D I_{E 3, S 4}$ from Figure 3-4-13 (a) and Figure 3-4-13 (c).

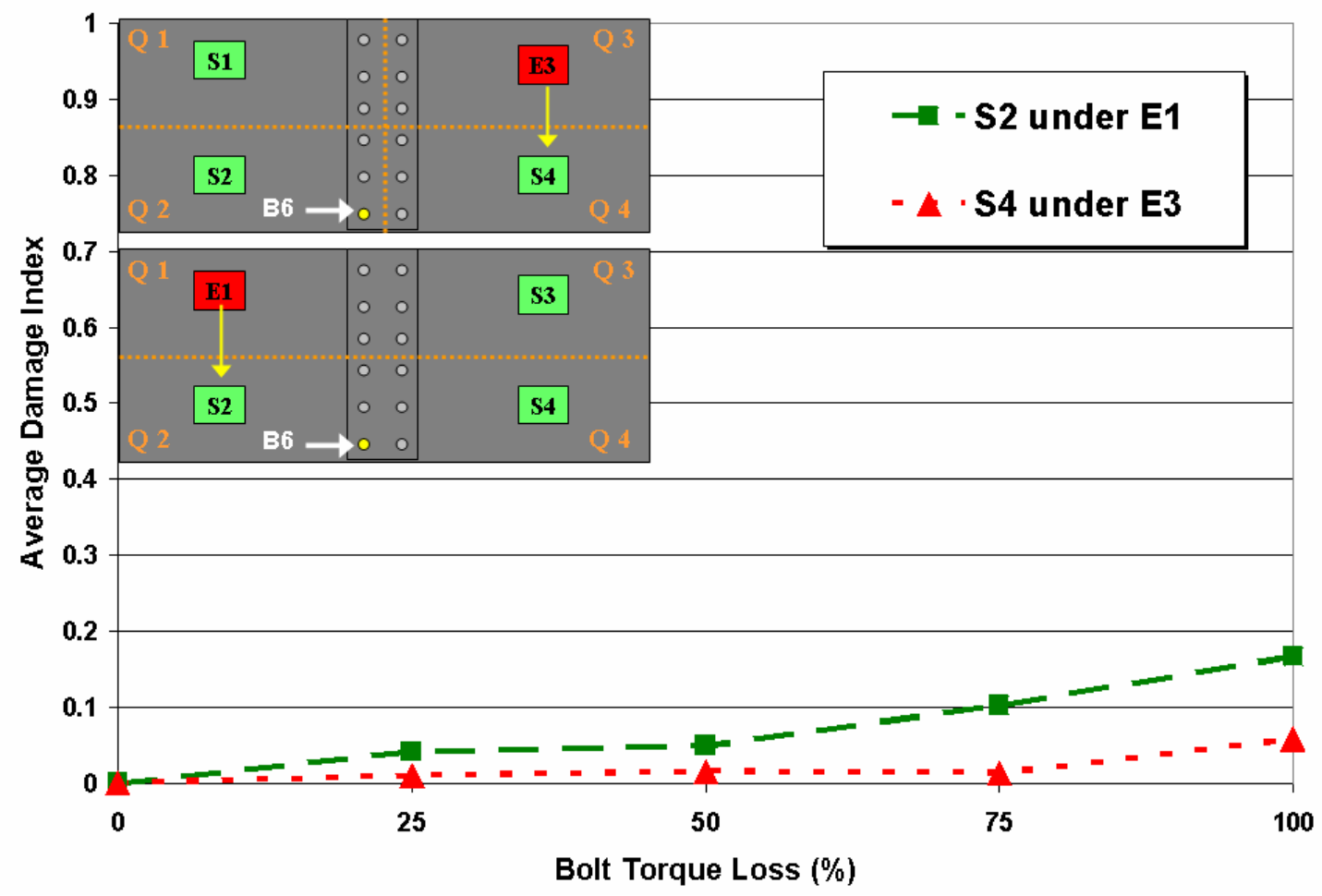

Figure 3-4-15. Comparison of normalized average damage indices, B6 torque loss; $D I_{E 1, S 2}$ and $D I_{E 3, S 4}$.

Comparing damage index information using the excitation configurations on the same composites as the sensors, a difference in trends was clearer. Figure 3-4-15 shows a higher average $D I$ at $\mathrm{S} 3$ compared to $\mathrm{S} 4$ at all values of Bolt 6 torque loss. Through this relationship, the ambiguity from Figure 3-4-14 was diminished and the estimated 
damage area was shown to be closer to S2, thus in Quadrant 2. Figure 3-4-16 shows the shaded area of the highest likelihood of damage.

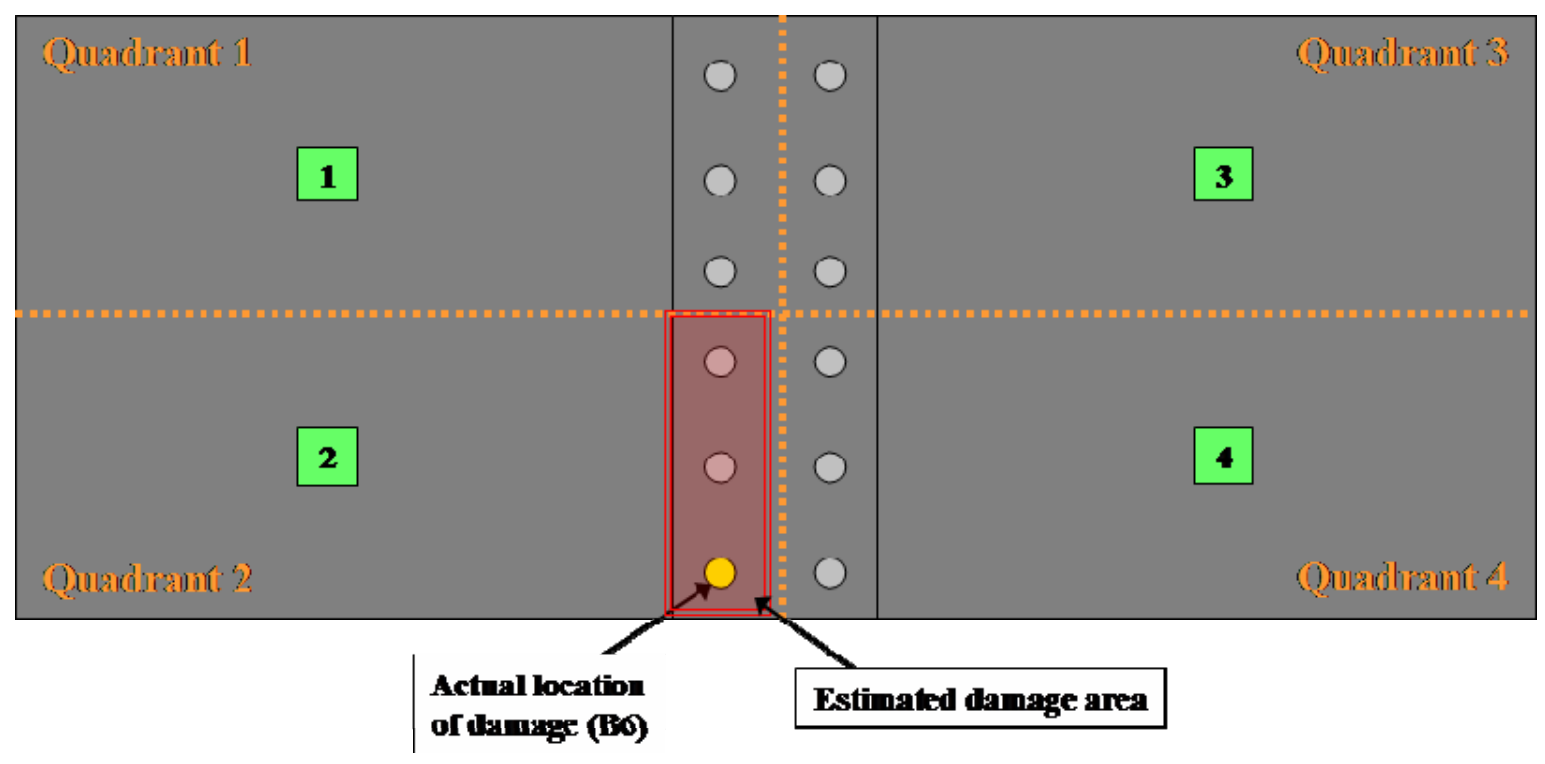

Figure 3-4-16. Estimated damage area due to B6 torque loss.

\section{$3.5 \quad$ Dual-bolt damage due to torque loss}

Previously, all experimentation has been performed with the isolation of a single bolt for damage due to torque loss. In this section, a multiple bolt damage scenario was examined to test the Lamb wave method sensitivity and performance. Two different bolt pairs were examined. The first pair consisted of two bolts within the same quadrant, Bolt 0 and Bolt 1, which are located in Quadrant 3. A second pair consisted of Bolt 0 and Bolt 6, which are located in different quadrants (Quadrant 3 and Quadrant 2) and are oriented on opposite sides of the composite joint. Bolt pairs underwent identical torque loss manipulation and were tested under each of the four excitation configuration cases. Results were compiled in the same manner as the single bolt experimentation and 
analysis, and the Lamb wave method was scrutinized as a damage detection and localization method for a multiple bolt damage scenario.

\subsubsection{Bolt 0 and Bolt 1, Excitation Case I}

Figure 3-5-1 shows the excitation and sensing configuration and indicates the Bolt 0 and Bolt 1 locations of damage due to torque loss. Figure 3-5-2 presents the damage index using the energy magnitudes due to frequency response $\left(D I_{f}\right)$ at S2, S3 and S4 under E1 excitation. Figure 3-5-3 presents the damage index using the signals in time $\left(D I_{t}\right)$ at $\mathrm{S} 2, \mathrm{~S} 3$ and $\mathrm{S} 4$ sensing locations. Similar to the single bolt scenarios, the damage detection capabilities using time and frequency response magnitudes are favorable under a dual-bolt damage scenario. Damage index is shown to be more sensitive to bolt torque variation, while its sensitivity to excitation frequency is less clear.

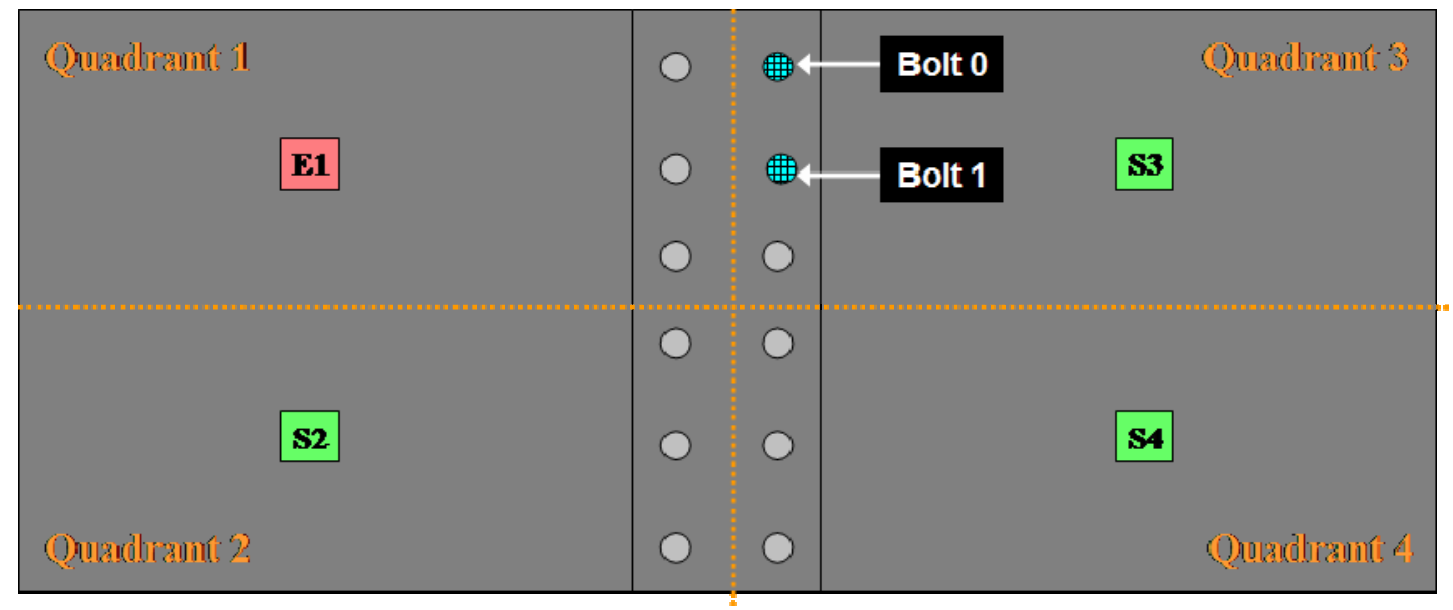

Figure 3-5-1. Excitation Case I, dual Bolt 0 and Bolt 1 variable torque loss. 


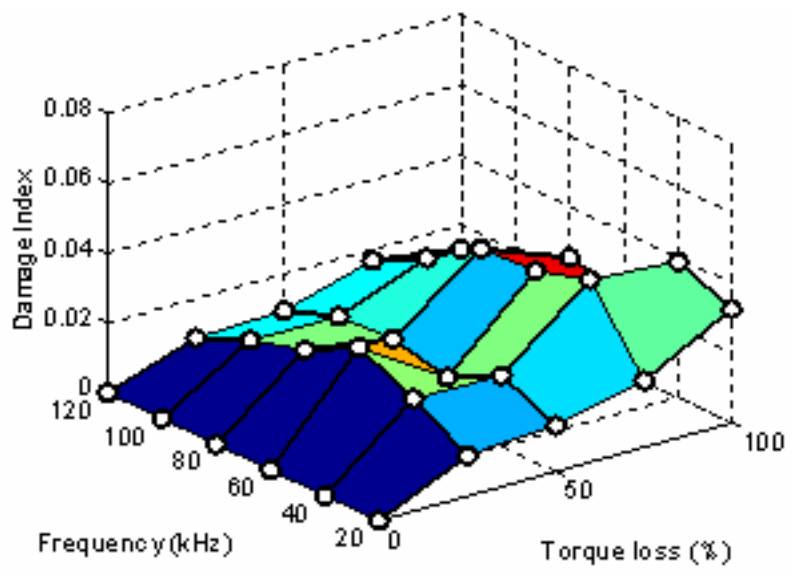

(a) $\mathrm{S} 2$ sensor

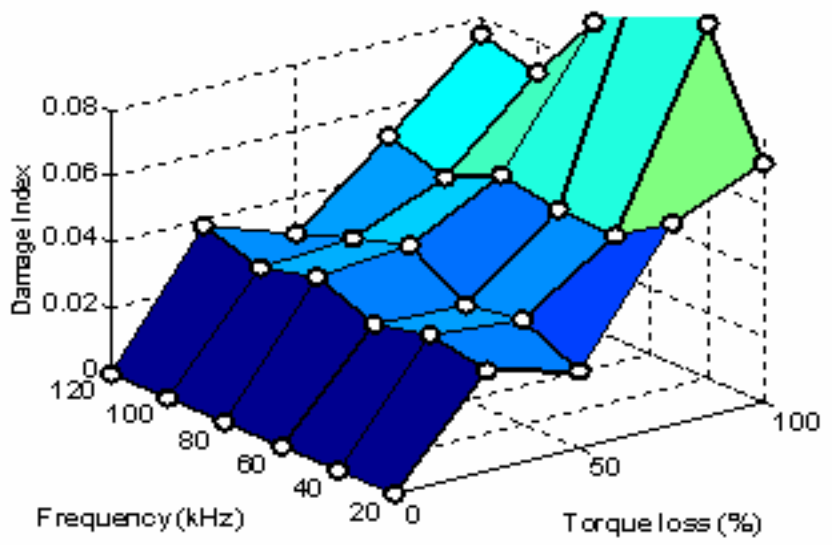

(b) S3 sensor

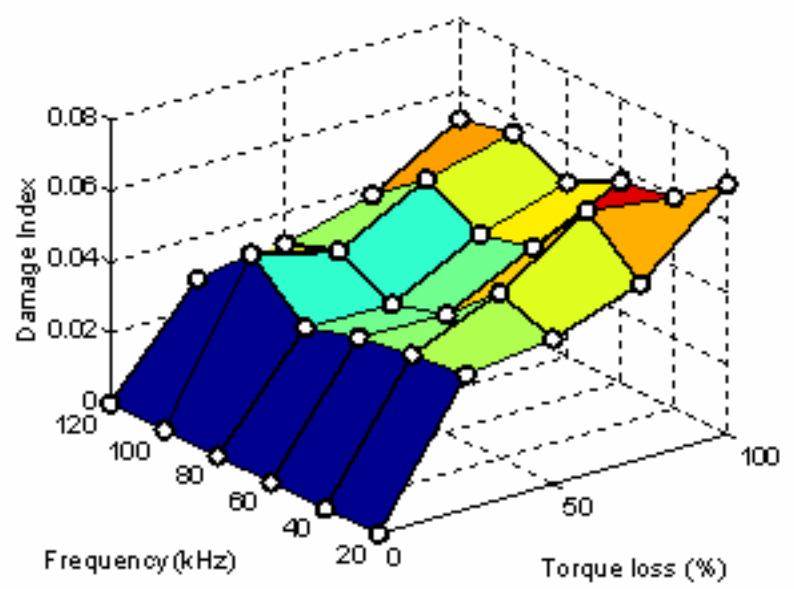

(c) S4 sensor

Figure 3-5-2. Damage index using the energy magnitudes in frequency vs. B0 and B1 torque loss and excitation frequency, E1 excitation. 


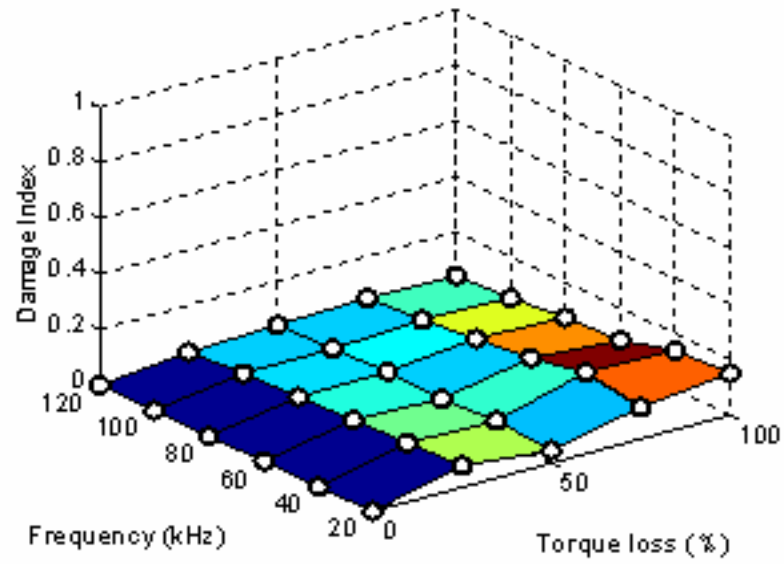

(a) $\mathrm{S} 2$ sensor

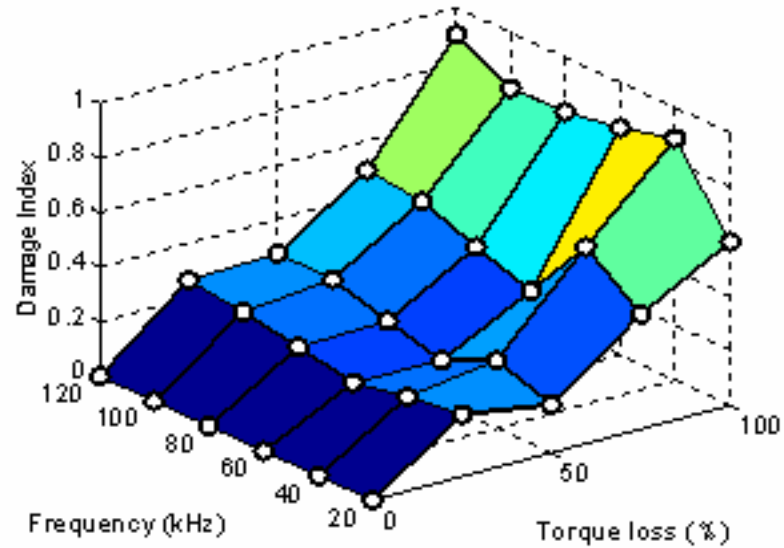

(b) S3 sensor

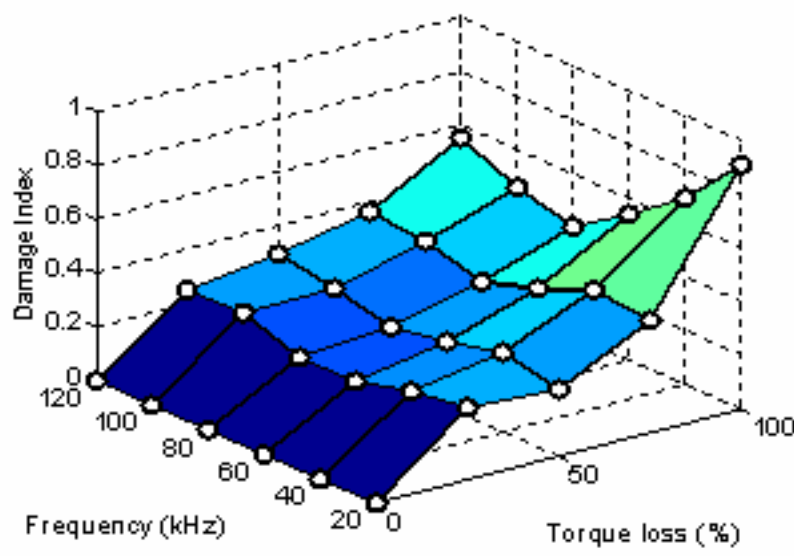

(c) S4 sensor

Figure 3-5-3. Damage index using the signals in time vs. B0 and B1 torque loss and excitation frequency, E1 excitation 


\subsubsection{Bolt 0 and Bolt 1, Excitation Case II}

Figure 3-5-4 shows the excitation and sensing configuration and indicates the Bolt 0 and Bolt 1 locations of damage due to torque loss. Figure 3-5-5 presents the damage index using the energy magnitudes due to frequency response $\left(D I_{f}\right)$ at S1, S3 and S4 under E2 excitation. Figure 3-5-6 presents the damage index using the signals in time $\left(D I_{t}\right)$ at $\mathrm{S} 1, \mathrm{~S} 3$ and $\mathrm{S} 4$ sensing locations. The damage index under E2 excitation displays a similar sensitivity to bolt torque loss and excitation frequency as compared to the E1 excitation configuration in the previous sub-section.

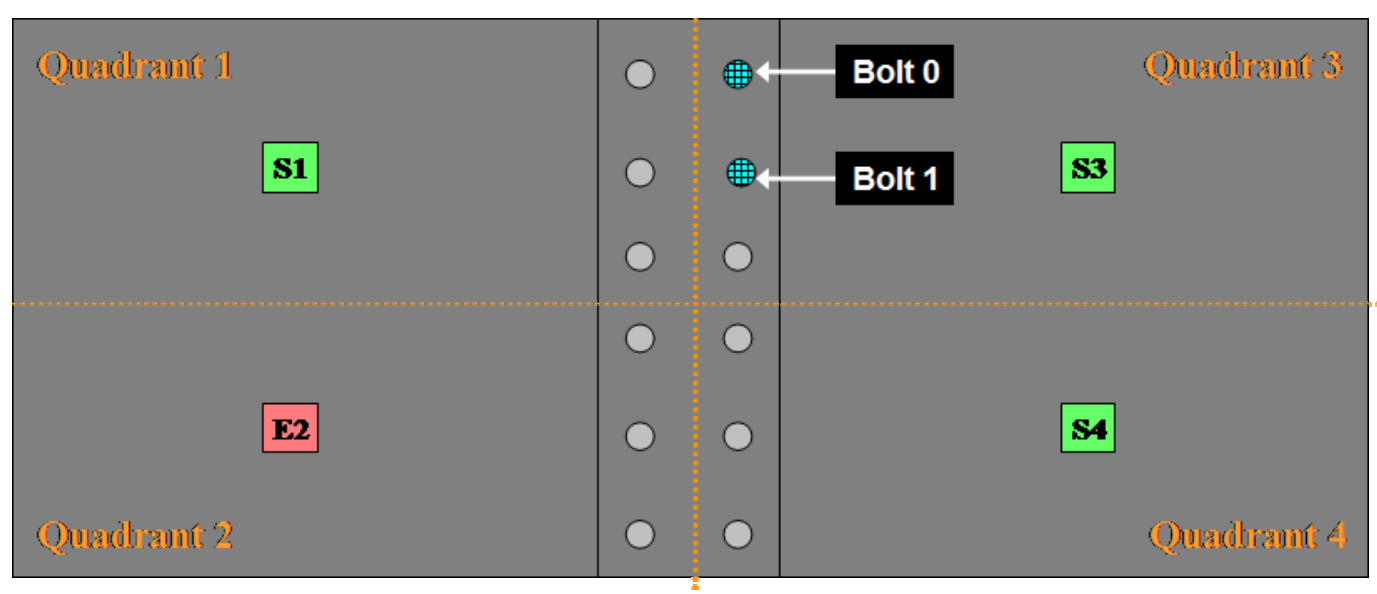

Figure 3-5-4. Excitation Case II, dual Bolt 0 and Bolt 1 variable torque loss. 


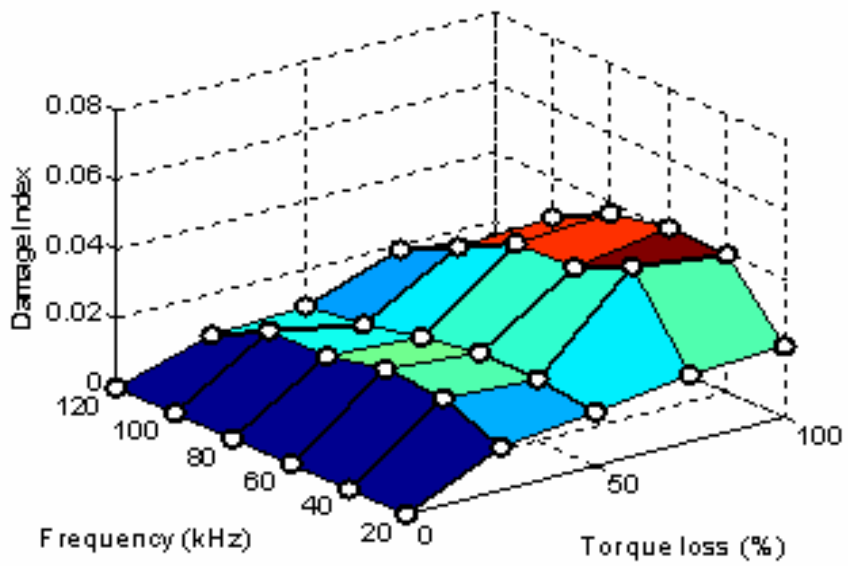

(a) S1 sensor

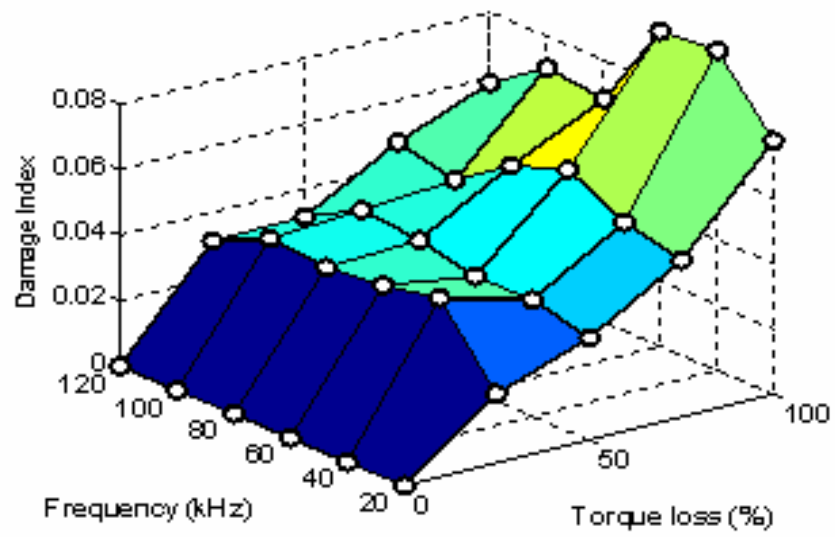

(b) S3 sensor

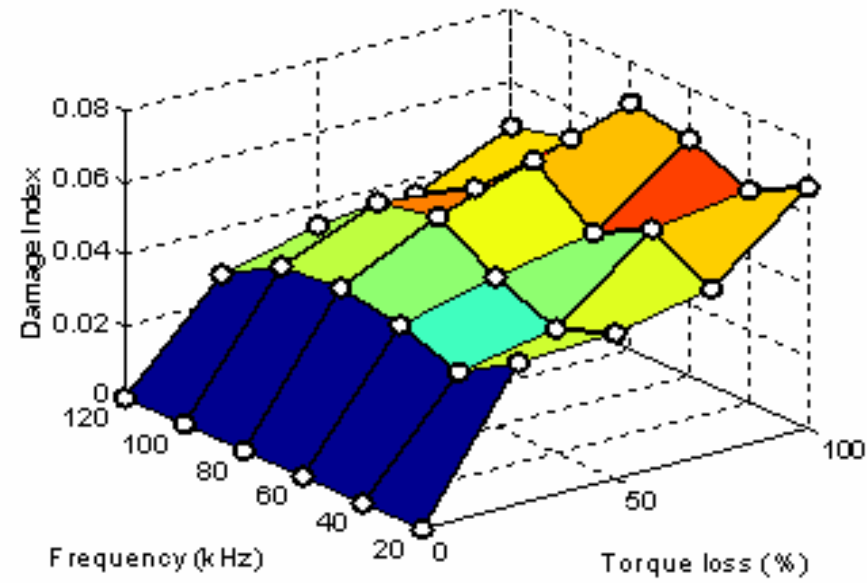

(c) $\mathrm{S} 4$ sensor

Figure 3-5-5. Damage index using the energy magnitudes in frequency vs. B0 and B1 torque loss and excitation frequency, E2 excitation. 


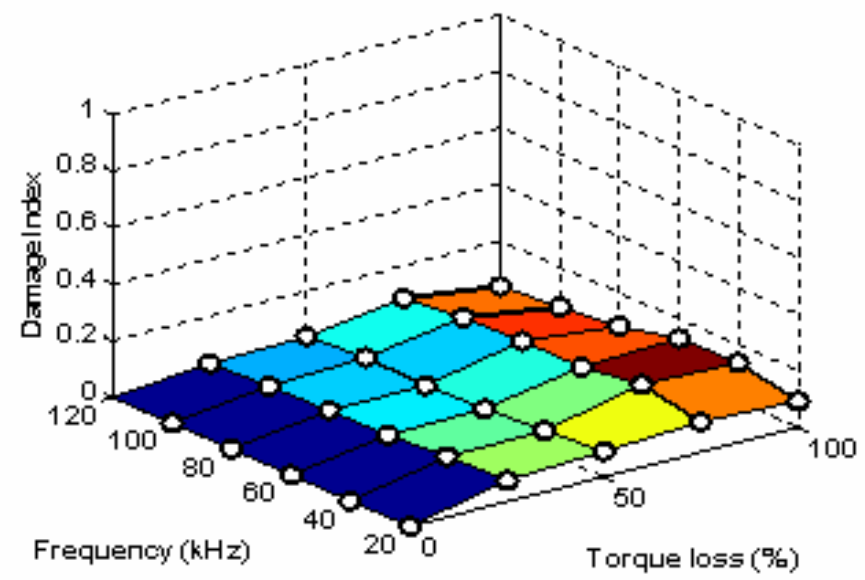

(a) S1 sensor

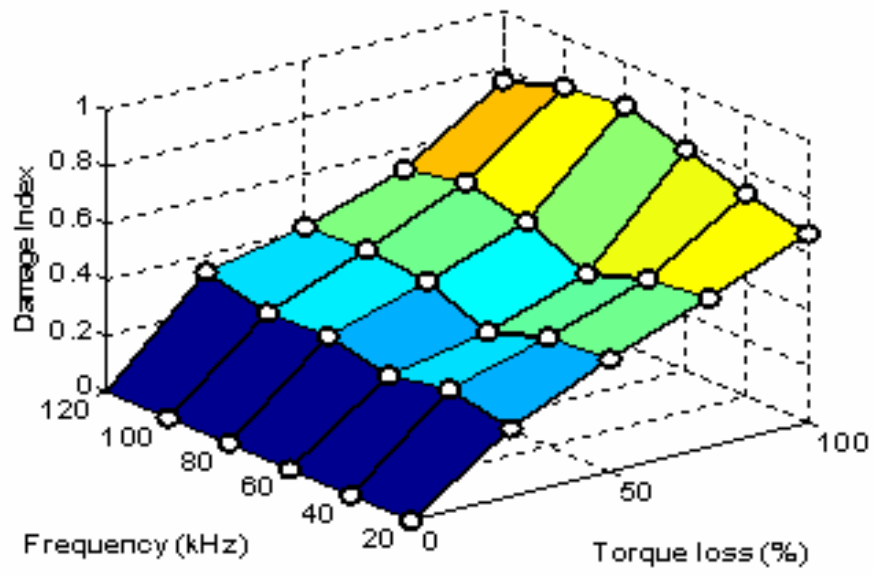

(b) S3 sensor

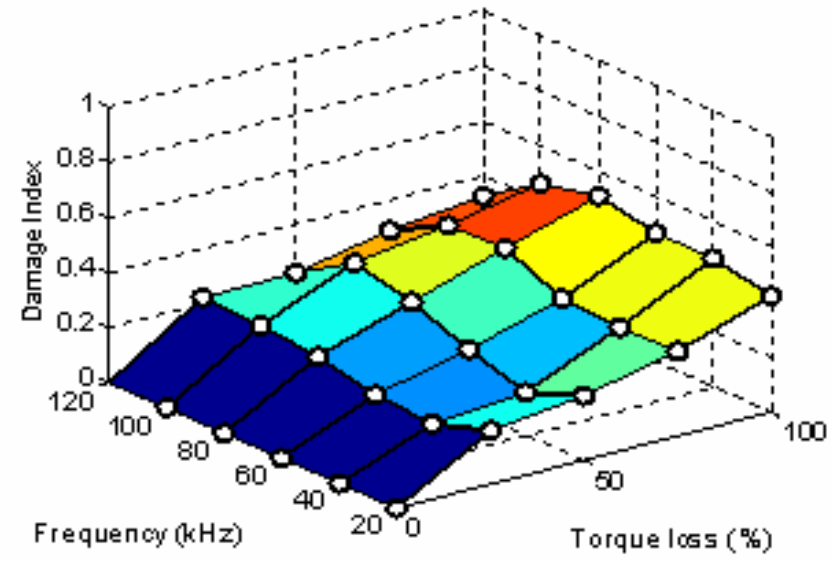

(c) S4 sensor

Figure 3-5-6. Damage index using the signals in time vs. B0 and B1 torque loss and excitation frequency, E2 excitation. 


\subsubsection{Bolt 0 and Bolt 1, Excitation Case III}

Figure 3-5-7 shows the excitation and sensing configuration and indicates the Bolt 0 and Bolt 1 locations of damage due to torque loss. In this excitation case, the origin of actuation was located within the same quadrant as the implemented damage. Figure 3-5-8 presents the damage index using the energy magnitudes due to frequency response $\left(D I_{f}\right)$ at $\mathrm{S} 1, \mathrm{~S} 2$ and $\mathrm{S} 4$ under E3 excitation. Figure 3-5-9 presents the damage index using the signals in time $\left(D I_{t}\right)$ at $\mathrm{S} 1, \mathrm{~S} 2$ and $\mathrm{S} 4$ sensing locations. There was a quantifiable damage index for each sensor over the range of excitation frequencies, which indicates favorable damage detection capability using time and frequency response magnitudes under a dual-bolt damage scenario. The damage index under E3 excitation displays a similar sensitivity to bolt torque loss and excitation frequency as compared to the E1 and E2 excitation configurations, previously shown.

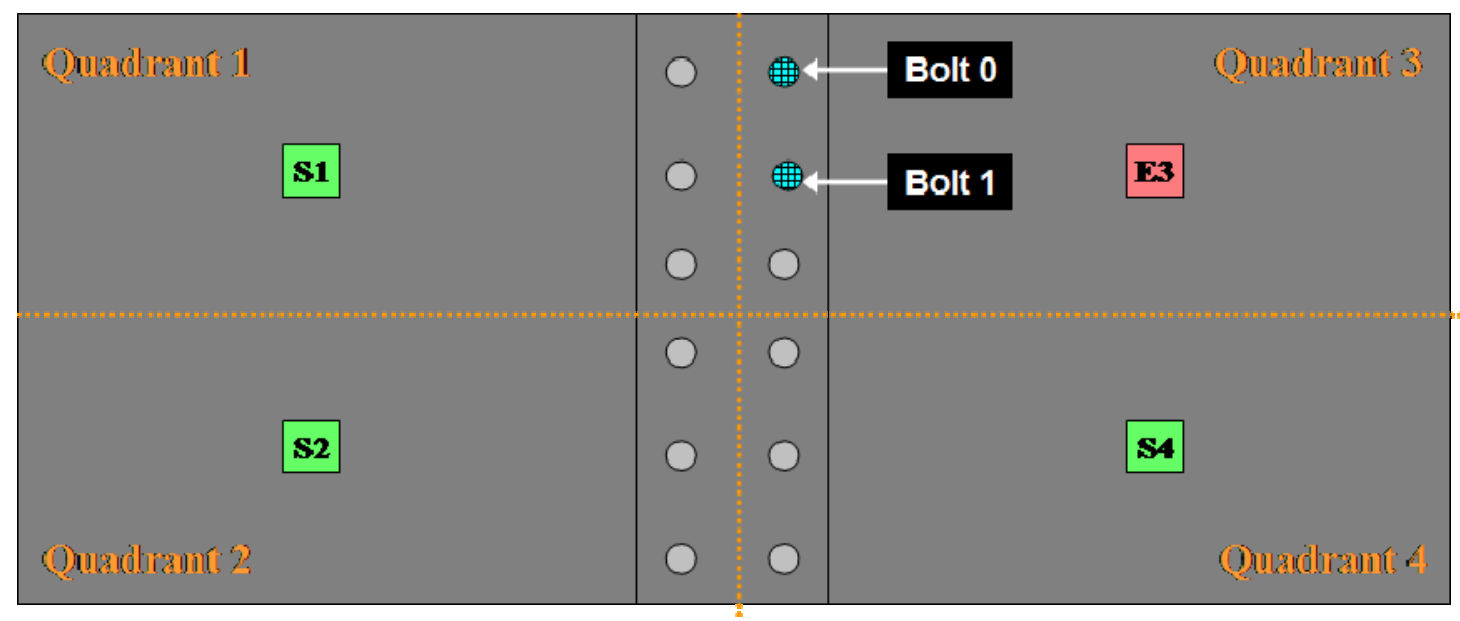

Figure 3-5-7. Excitation Case III, dual Bolt 0 and Bolt 1 variable torque loss. 


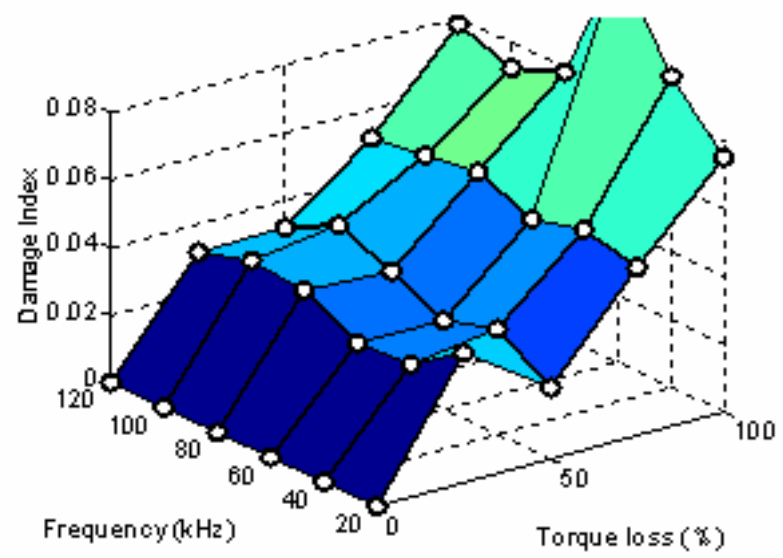

(a) S1 sensor

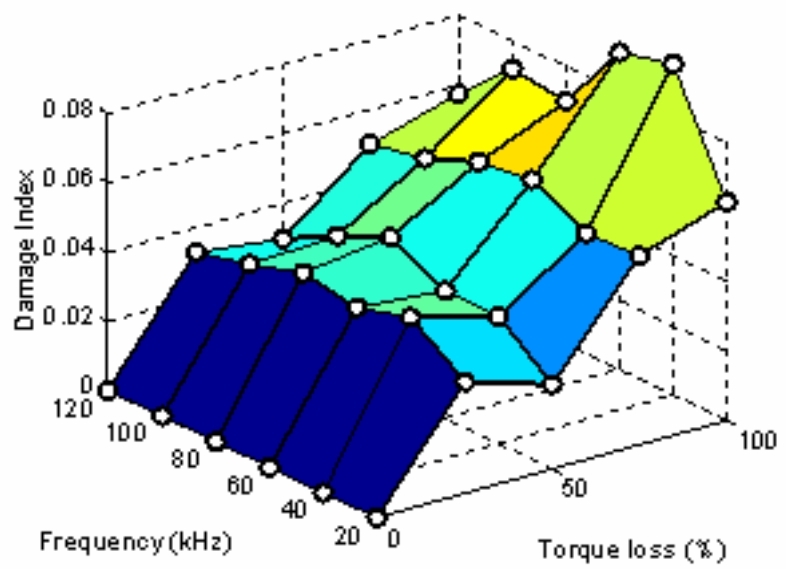

(b) $\mathrm{S} 2$ sensor

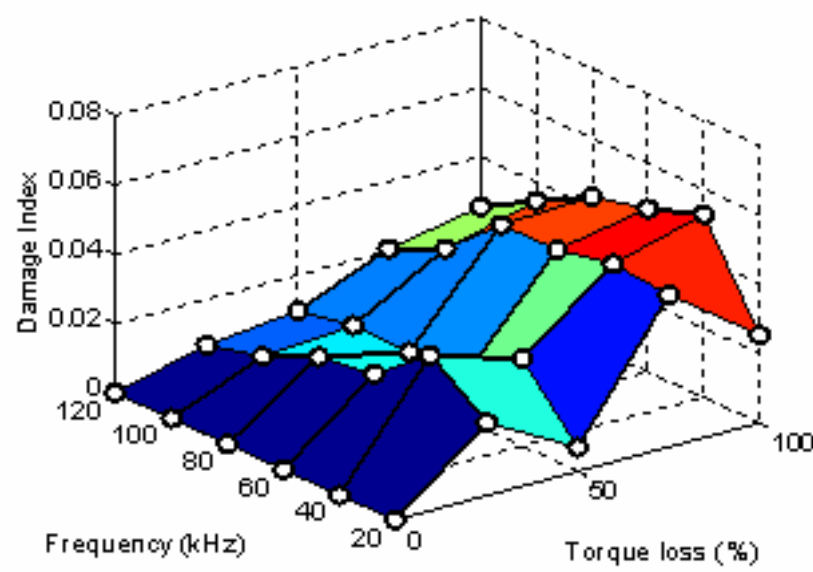

(c) $\$ 4$ sensor

Figure 3-5-8. Damage index using the energy magnitudes in frequency vs. B0 and B1 torque loss and excitation frequency, E3 excitation. 


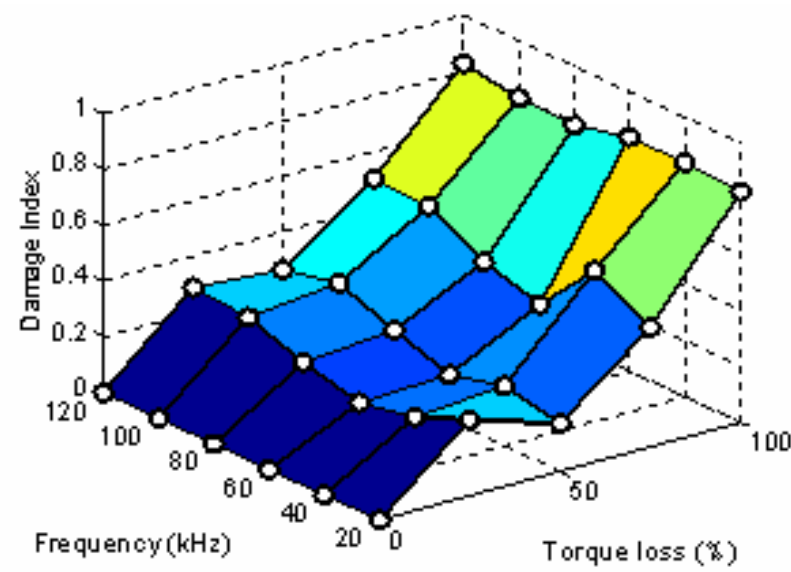

(a) S1 sensor

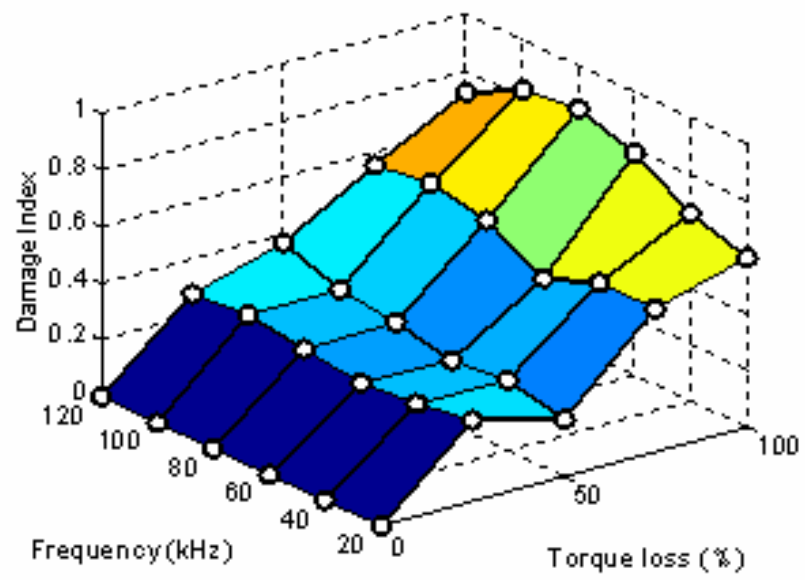

(b) S2 sensor

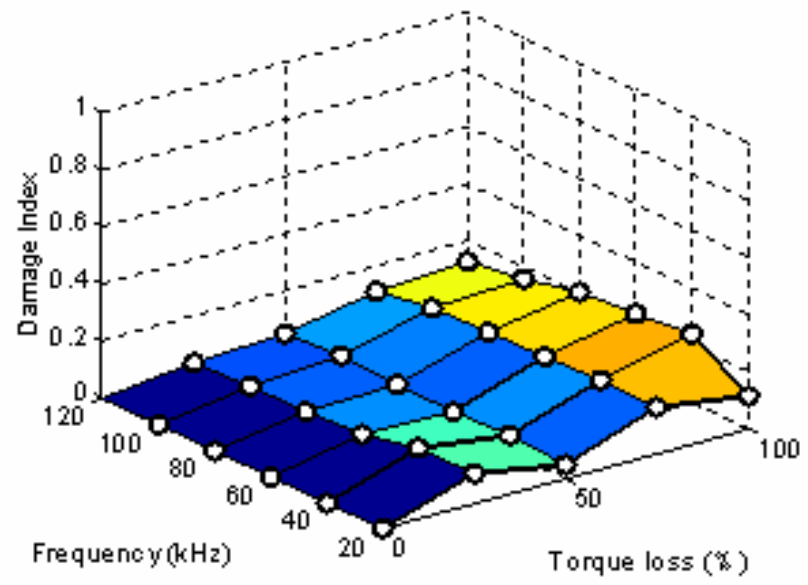

(c) $\$ 4$ sensor

Figure 3-5-9. Damage index using the signals in time vs. B0 and B1 torque loss and excitation frequency, E3 excitation. 


\subsubsection{Bolt 0 and Bolt 1, Excitation Case IV}

Figure 3-5-10 shows the fourth excitation and sensing configuration and indicates the Bolt 0 and Bolt 1 locations of damage due to torque loss. Figure 3-5-11 presents the damage index using the energy magnitudes due to frequency response $\left(D I_{f}\right)$ at $\mathrm{S} 1, \mathrm{~S} 2$ and S3 under E4 excitation. Figure 3-5-12 presents the damage index using the signals in time $\left(D I_{t}\right)$ at $\mathrm{S} 1, \mathrm{~S} 2$ and $\mathrm{S} 3$ sensing locations. The damage indices sensitivity to increasing torque loss remains clearer as compared to the sensitivity due to excitation frequency.

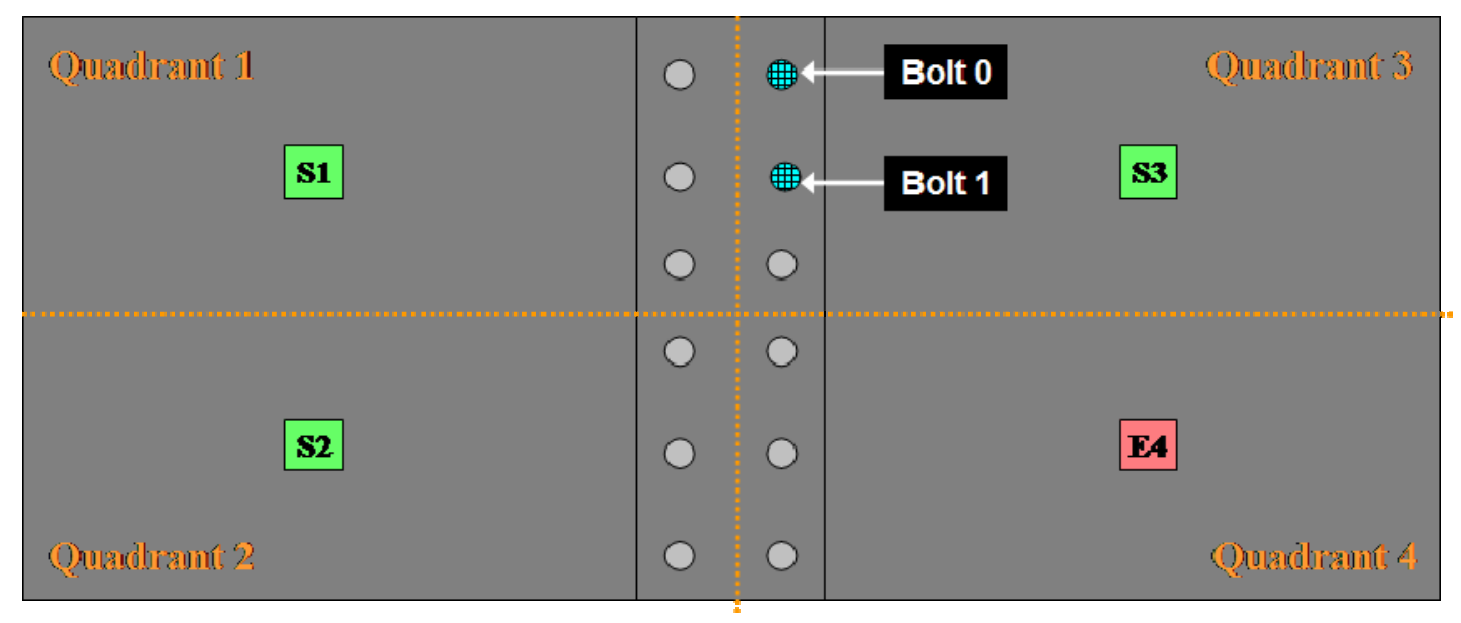

Figure 3-5-10. Excitation Case IV, dual Bolt 0 and Bolt 1 variable torque loss. 


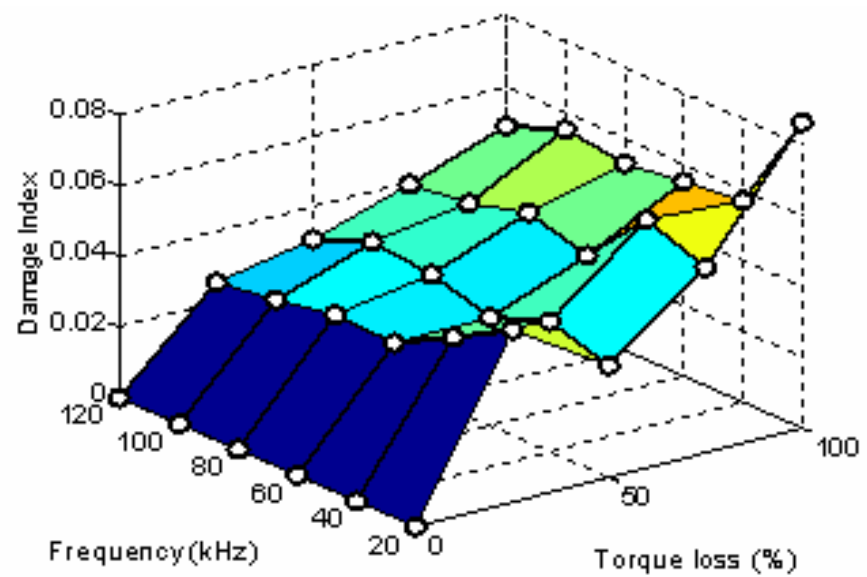

(a) S1 sensor

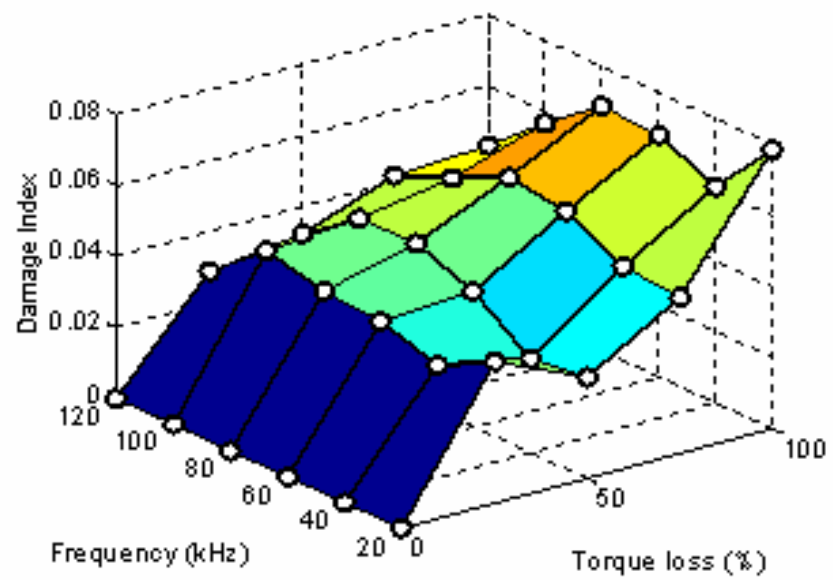

(b) $\mathrm{S} 2$ sensor

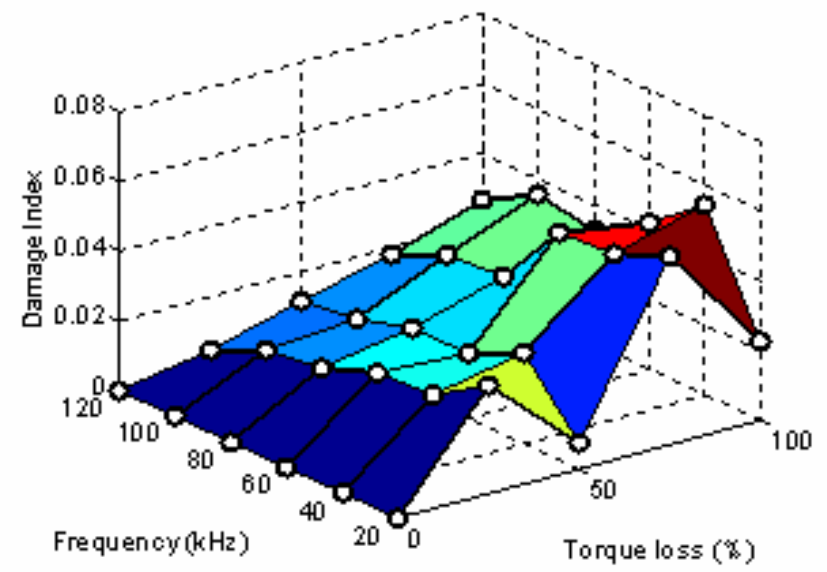

(c) S3 sensor

Figure 3-5-11. Damage index using the energy magnitudes in frequency vs. B0 and B1 torque loss and excitation frequency, E4 excitation. 


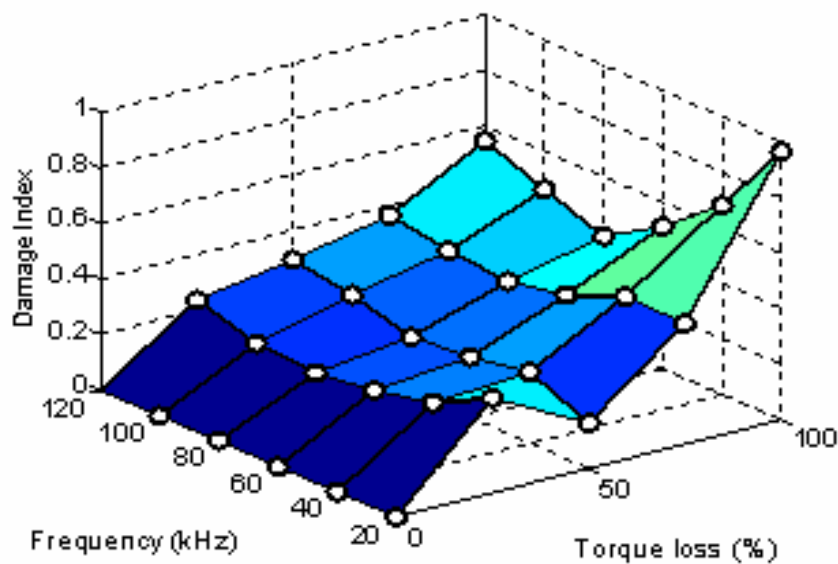

(a) S1 sensor

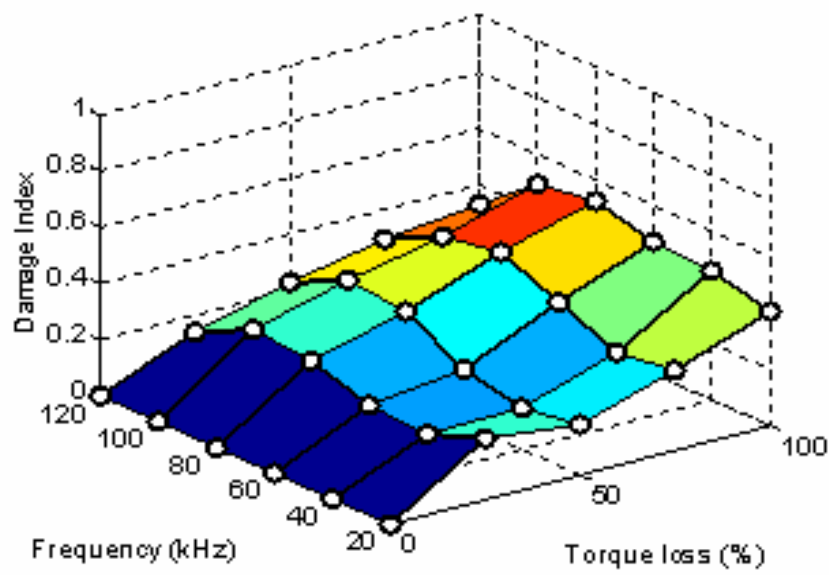

(b) S2 sensor

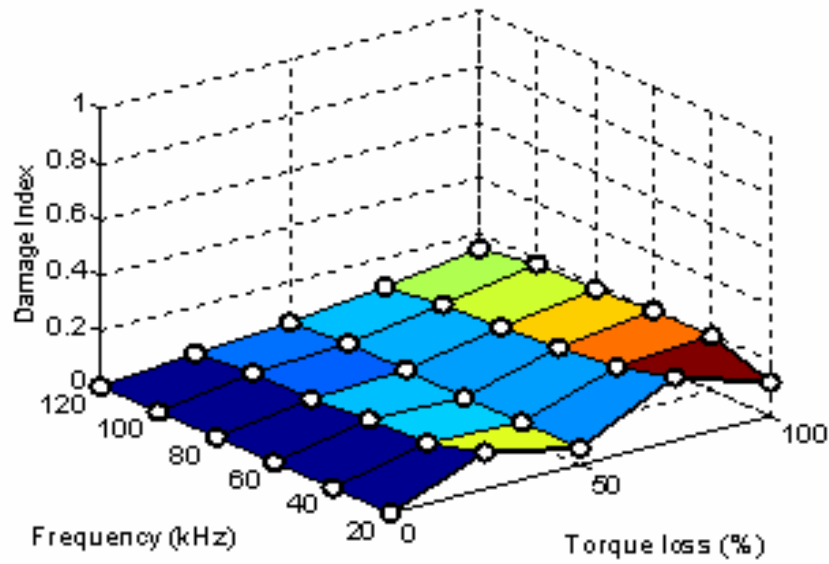

(c) S3 sensor

Figure 3-5-12. Damage index using the signals in time vs. B0 and B1 torque loss and excitation frequency, E4 excitation. 


\subsubsection{Comparison of dual Bolt 0 and Bolt 1 excitation configurations}

Figure 3-5-13 shows the comparison of the normalized average damage index of the composite jointed panel due to dual-bolt torque loss within Bolt 0 and Bolt 1 for each excitation configuration. The Lamb wave method exhibited favorable sensitivity to damage in all excitation cases, as indicated by the average damage index quantities at all values of torque loss. Additionally, the damage indices sensitivity to increasing bolt torque loss was higher than the sensitivity due to increasing excitation frequency; this was the case for all excitation configurations. The ability of the Lamb wave method to localize damage under a dual-bolt damage scenario was examined in the same manner as the single-bolt damage cases. The following analysis was made to provide a rough estimate of the location of damage due to torque loss:

$$
\begin{aligned}
& D I_{E l, S 3}>D I_{E 1, S 4}>>D I_{E l, S 2} \quad \text { Figure 3-5-13 (a) } \\
& D I_{E 2, S 3}>D I_{E 2, S 4}>>D I_{E 2, S 1} \quad \text { Figure 3-5-13 (b) } \\
& D I_{E 3, S 1} \approx D I_{E 3, S 2}>>D I_{E 3, S 4} \quad \text { Figure 3-5-13 (c) } \\
& D I_{E 4, S 1} \approx D I_{E 4, S 2}>>D I_{E 4, S 3} \quad \text { Figure 3-5-13 (d) }
\end{aligned}
$$

These relationships provided an estimation of damage within the area between sensors S1 and S1, thus within Quadrant 1 or Quadrant 3. The average damage index values under this dual-bolt damage scenario are capable of providing insight into the detection and localization of damage due to bolt torque loss within the composite joint. 


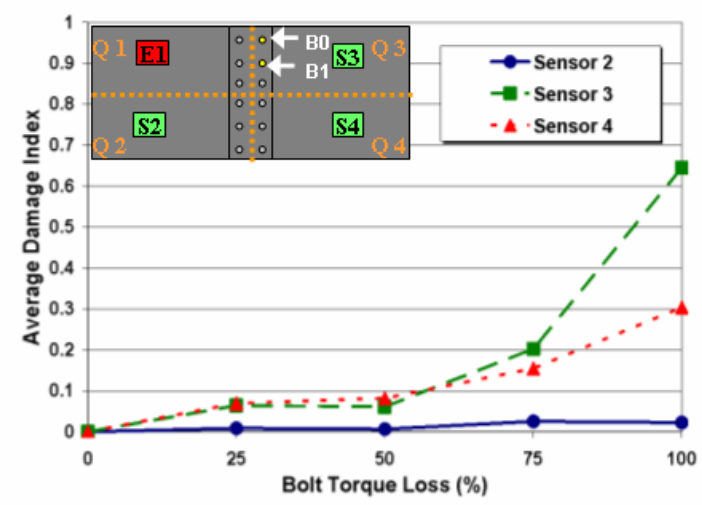

(a) E1 excitation

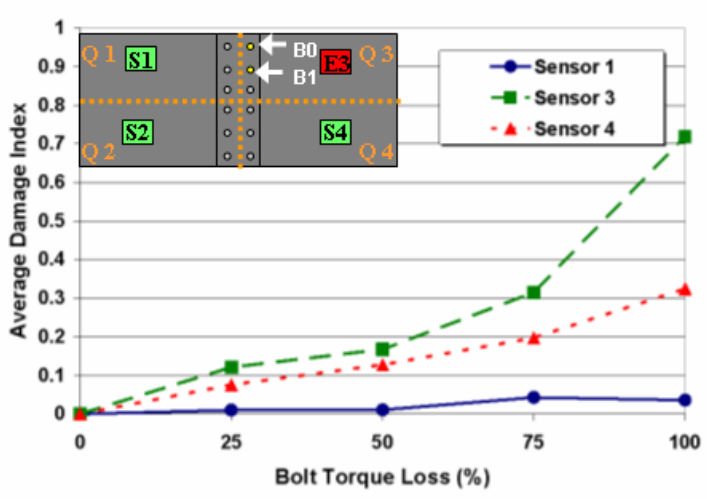

(c) E3 excitation

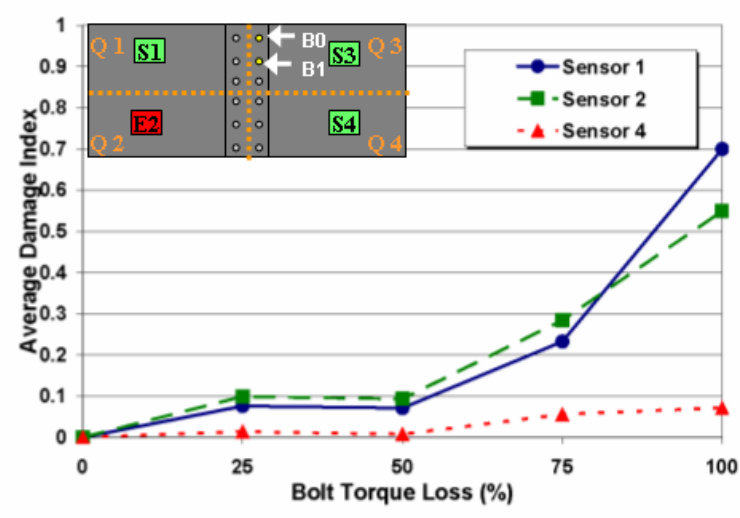

(b) E2 excitation

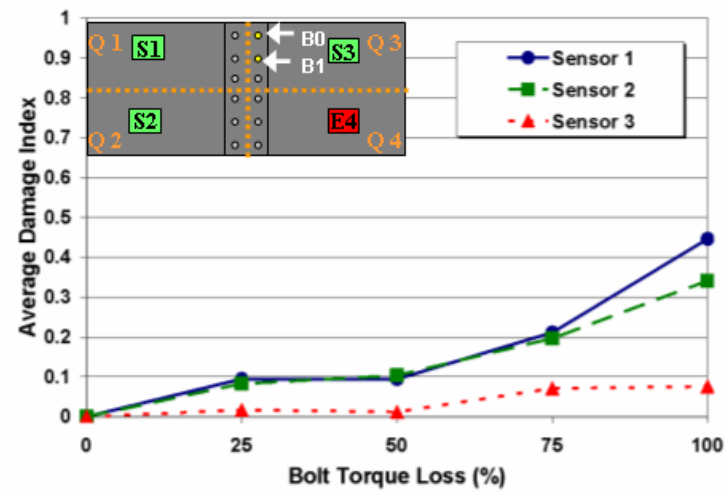

(d) E4 excitation

Figure 3-5-13. Comparison of normalized average damage index, Bolt 0 and Bolt 1 dualbolt torque loss.

To examine these trends in a similar manner to single-bolt damage scenarios in Quadrant 3, a comparison between the information in Figure 3-5-13 (b) and (d) was performed using the sensing information at S3 and S1, respectively. Comparing the $D I_{E 4, S 1}$ and $D I_{E 2, S 3}$ from Figure 3-5-13 (b) and Figure 3-5-13 (d) clearly shows:

$$
D I_{E 2, S 3}>D I_{E 4, S 1}
$$

The information is isolated to show this comparison graphically in Figure 3-5-14. 


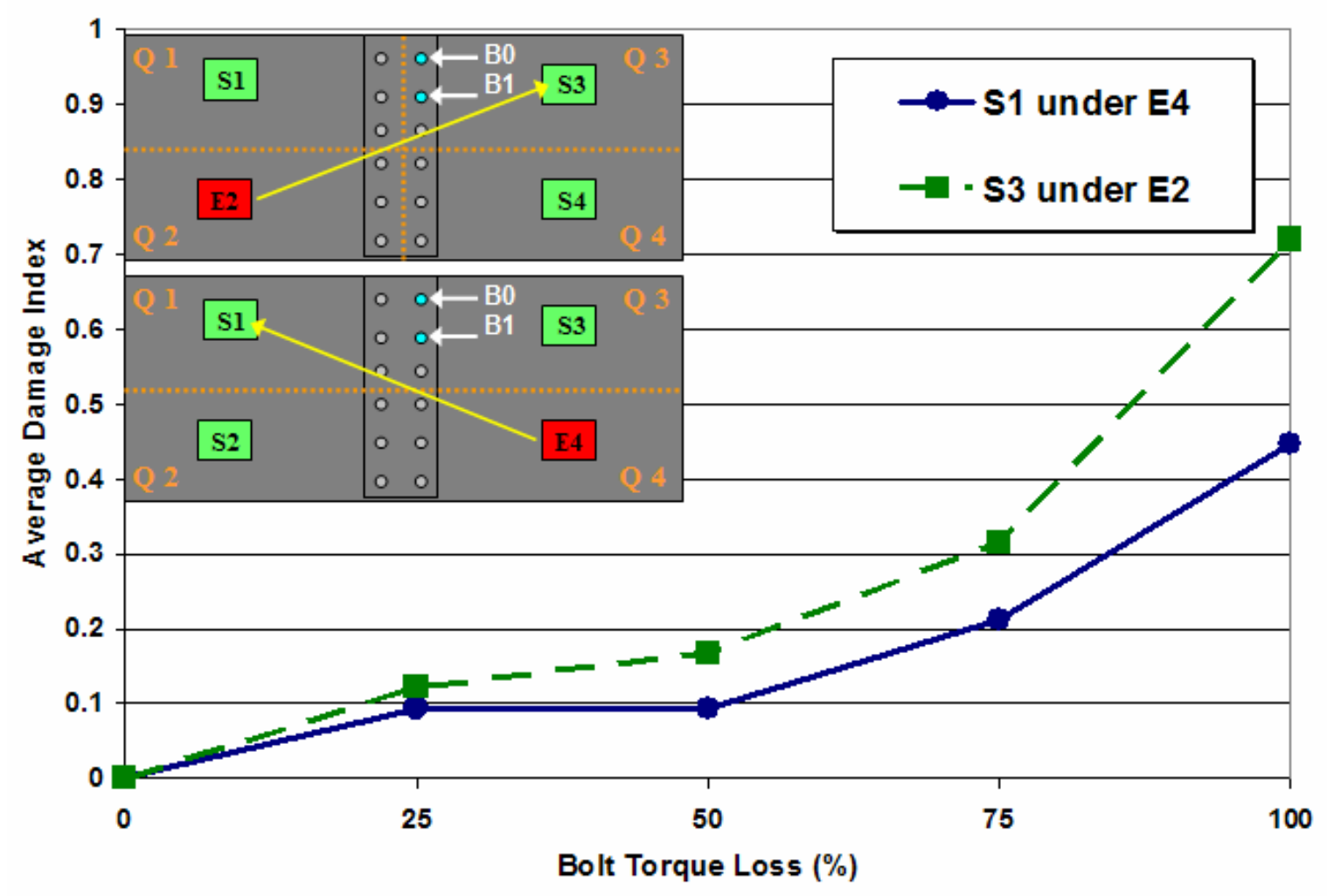

Figure 3-5-14. Comparison of normalized average damage indices, Bolt 0 and Bolt 1 torque loss; $D I_{E 4, S l}$ and $D I_{E 2, S 3}$.

A similar result to the single-bolt damage scenarios, this dual-bolt damage trend indicates a higher damage index within proximity to $S 3$, thus the greater likelihood of damage due to bolt torque loss in Quadrant 3 as compared to Quadrant 1. Figure 3-5-15 shows the shaded area of the highest likelihood of damage. 


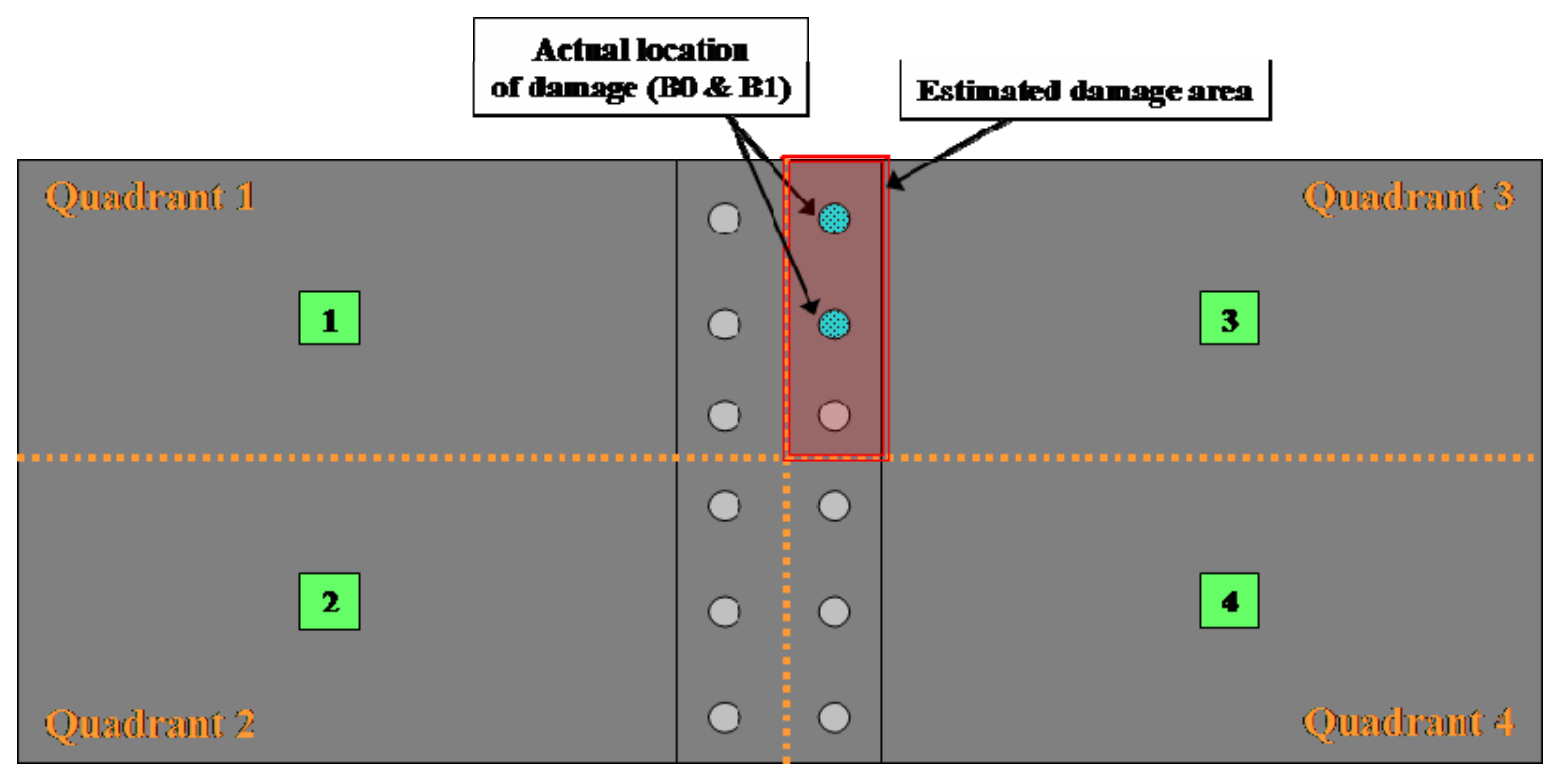

Figure 3-5-15. Estimated damage area due to Bolt 0 and Bolt 1 torque loss.

\subsubsection{Bolt 0 and Bolt 6, Excitation Case I}

Figure 3-5-16 shows the excitation and sensing configuration and indicates the Bolt 0 and Bolt 6 locations of damage due to torque loss. In this scenario, damage was implemented in two different quadrants, Quadrants 2 and 3 on opposite sides of the bolted composite joint. Previously, damage had always been in-between a single bolt sensing pair; in this case, damage was located in-between sensor pair S1 and S3 as well as in-between sensor pair S2 and S4. Figure 3-5-17 presents the damage index using the energy magnitudes due to frequency response $\left(D I_{f}\right)$ at S2, S3 and S4 under E1 excitation. Figure 3-5-18 presents the damage index using the signals in time $\left(D I_{t}\right)$ at $\mathrm{S} 2, \mathrm{~S} 3$ and $\mathrm{S} 4$ sensing locations. For each sensor and over the complete range of excitation frequencies, a quantifiable damage index due to time and frequency responses is shown, indicating favorable damage detection capabilities under this new dual-bolt damage scenario. 


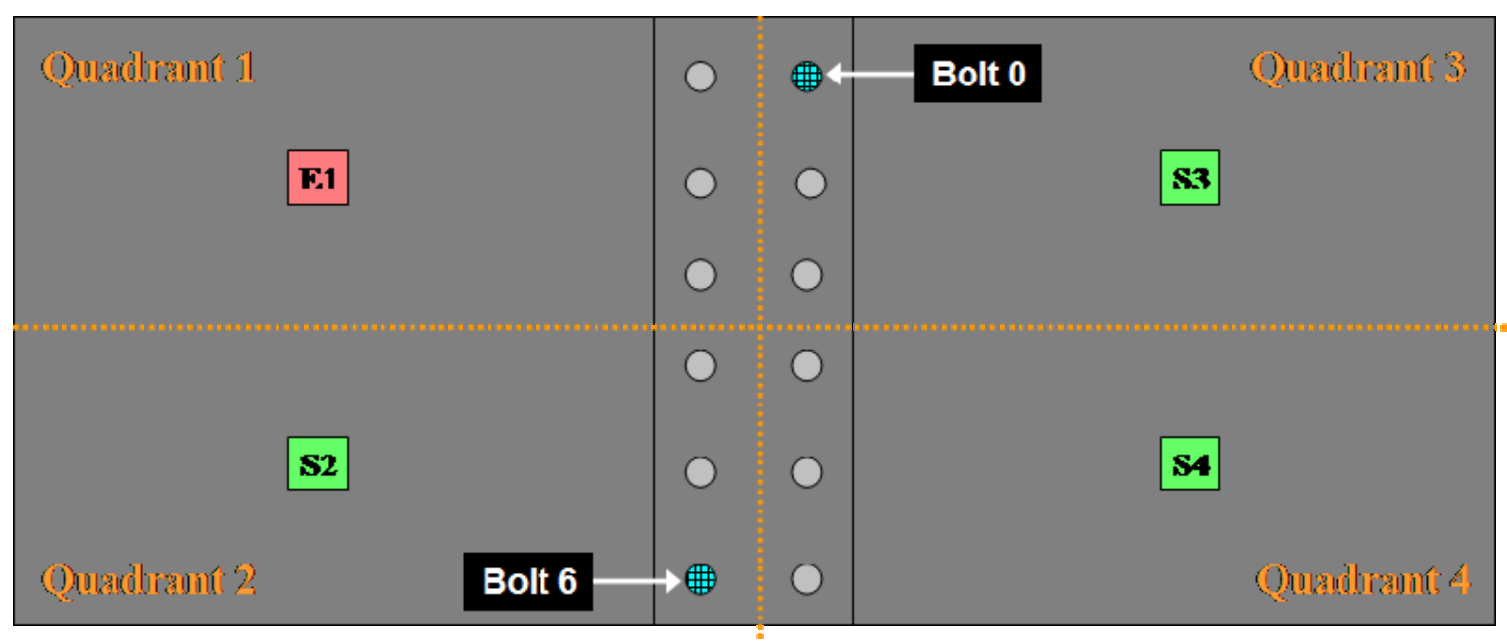

Figure 3-5-16. Excitation Case I, dual Bolt 0 and Bolt 6 variable torque loss. 


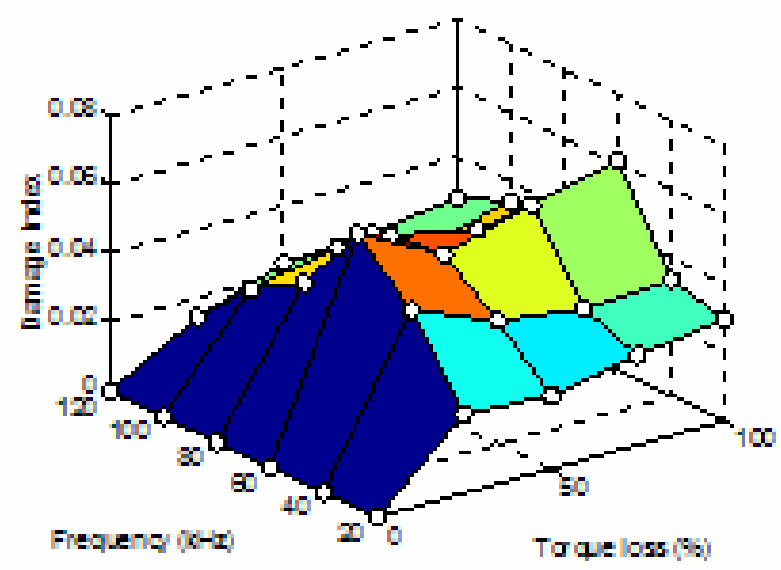

(a) $\mathrm{S} 2$ sensor

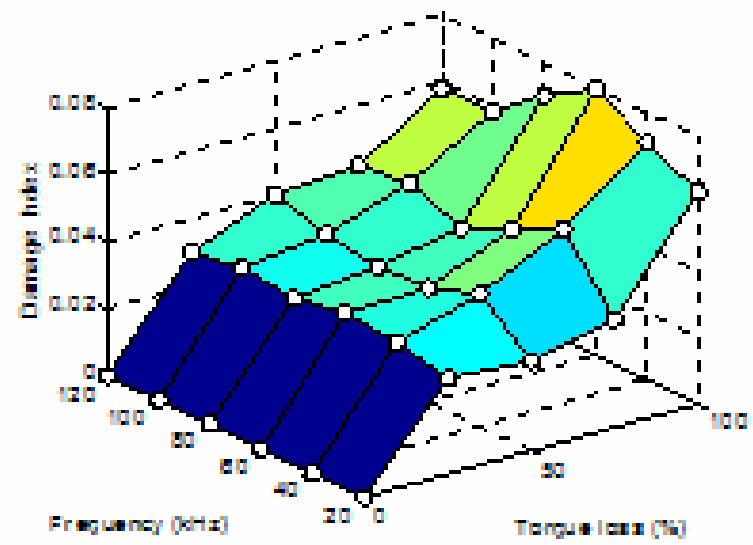

(b) S3 sensor

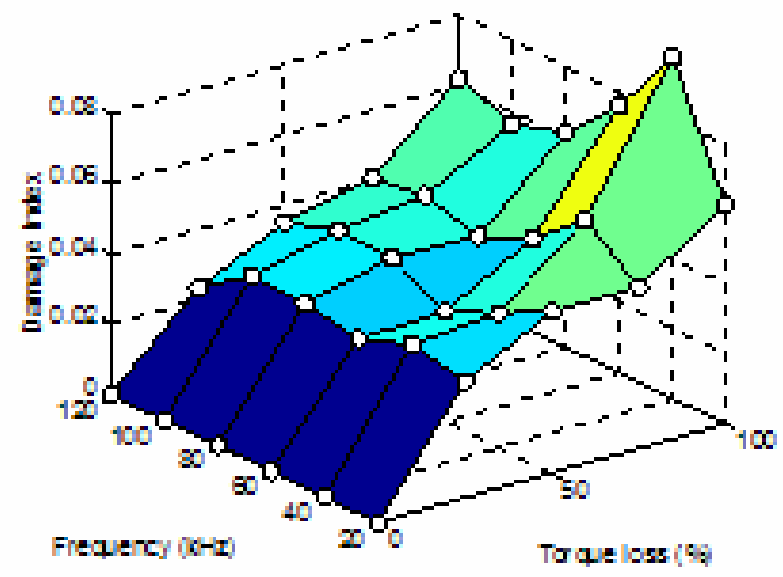

(c) S4 sensor

Figure 3-5-17. Damage index using the energy magnitudes in frequency vs. B0 and B6 torque loss and excitation frequency, E1 excitation. 


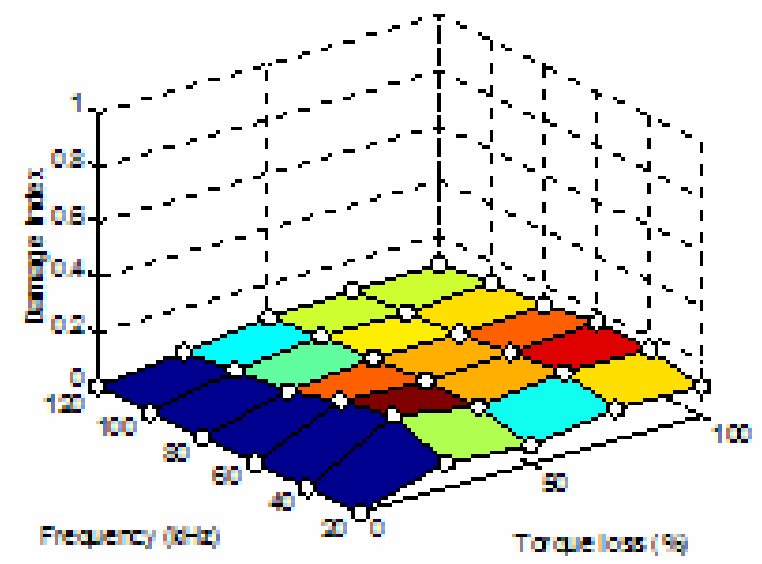

(a) S2 sensor

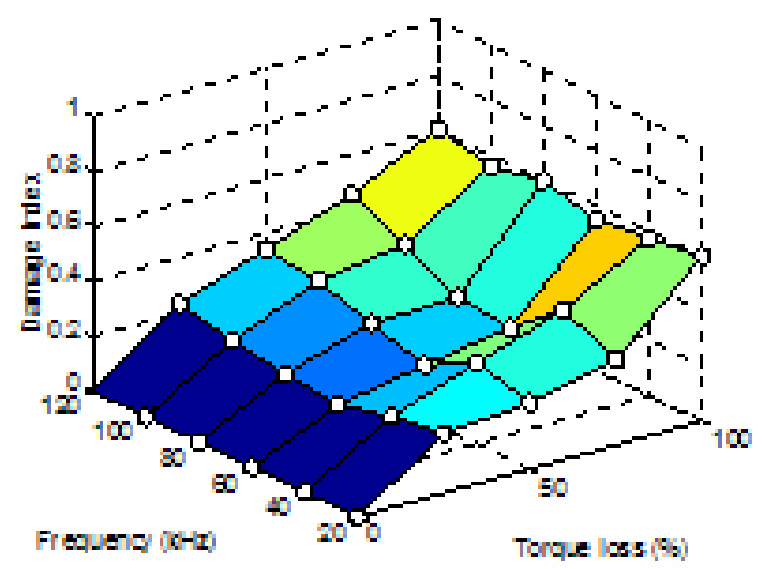

(b) $\mathrm{S3}$ sensor

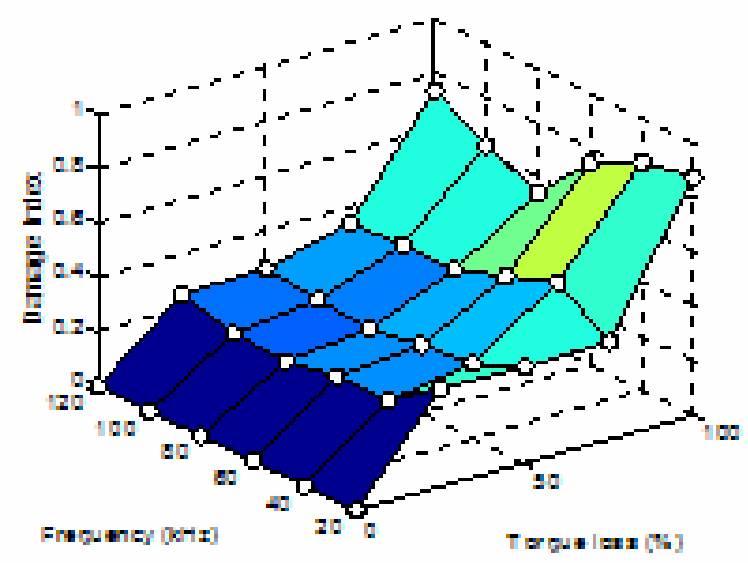

(c) $\mathrm{S4}$ sensor

Figure 3-5-18. Damage index using the signals in time vs. B0 and B6 torque loss and excitation frequency, E1 excitation. 


\subsubsection{Bolt 0 and Bolt 6, Excitation Case II}

Figure 3-5-19 shows the excitation and sensing configuration and indicates the Bolt 0 and Bolt 6 locations of damage due to torque loss. In this scenario, excitation occurred in one of the quadrants of damage, Quadrant 2, close to Bolt 6. Figure 3-5-20 presents the damage index using the energy magnitudes due to frequency response $\left(D I_{f}\right)$ at $\mathrm{S} 1, \mathrm{~S} 3$ and S4 under E2 excitation. Figure 3-5-21 presents the damage index using the signals in time $\left(D I_{t}\right)$ at $\mathrm{S} 1, \mathrm{~S} 3$ and $\mathrm{S} 4$ sensing locations. Quantifiable damage index due to time and frequency responses is shown, indicating favorable damage detection capabilities under a scenario where excitation was present in the same quadrant as damage due to torque loss of Bolt 6. In both plots the damage index trends display a higher sensitivity due to increasing bolt torque loss and a lower sensitivity across the range of excitation frequencies.

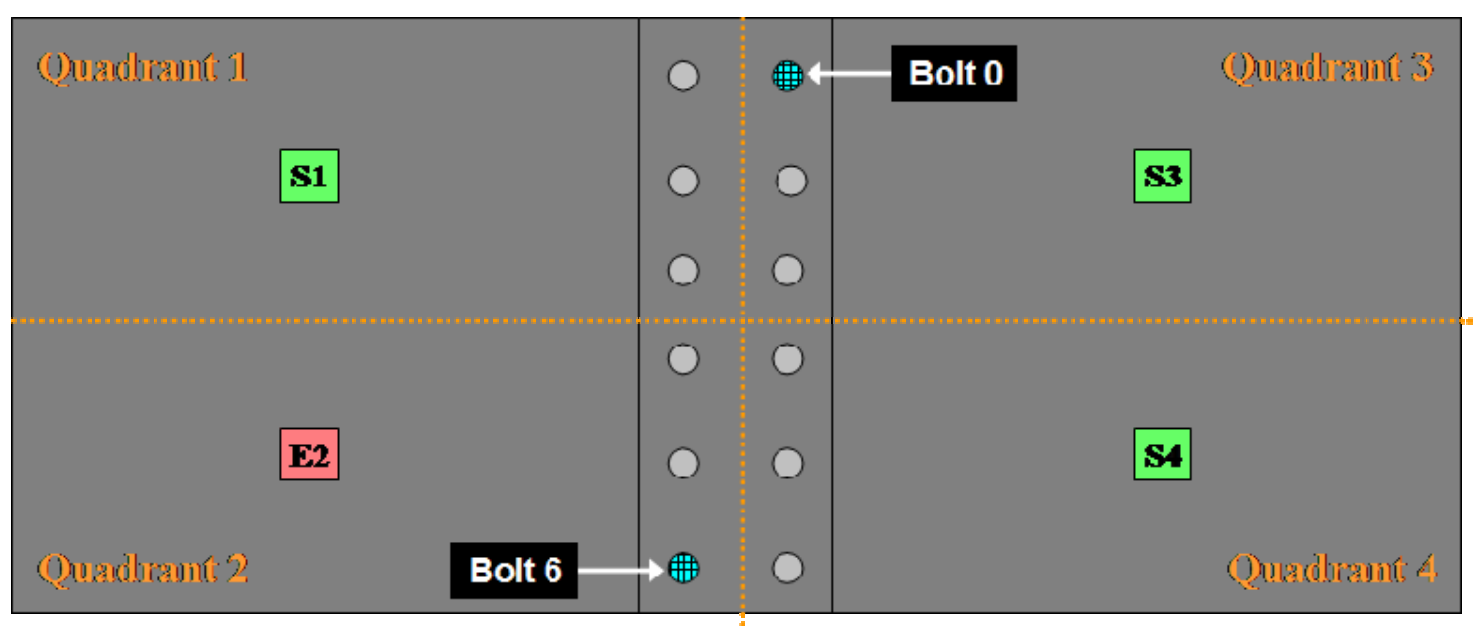

Figure 3-5-19. Excitation Case II, dual Bolt 0 and Bolt 6 variable torque loss. 


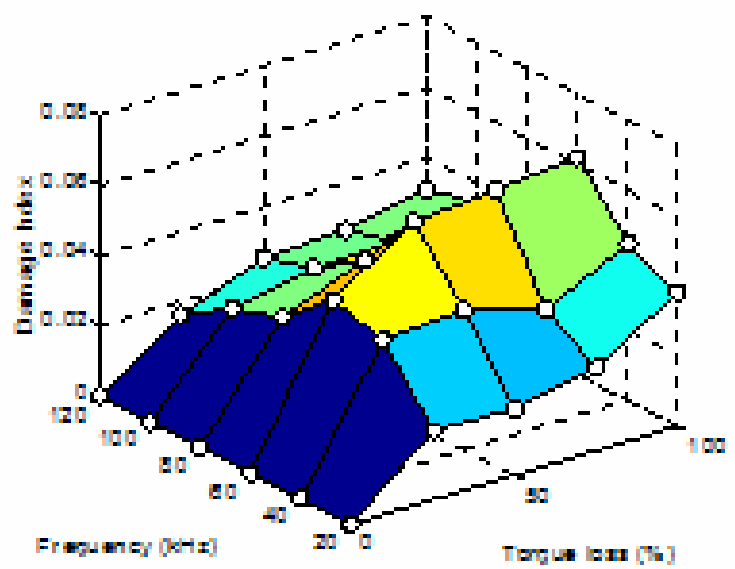

(a) $\mathrm{Sl}$ sensor

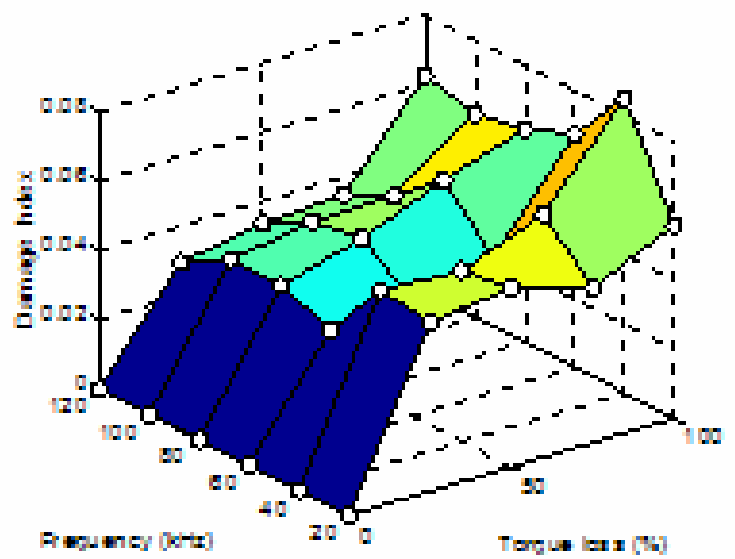

(b) $\mathrm{S3}$ sensor

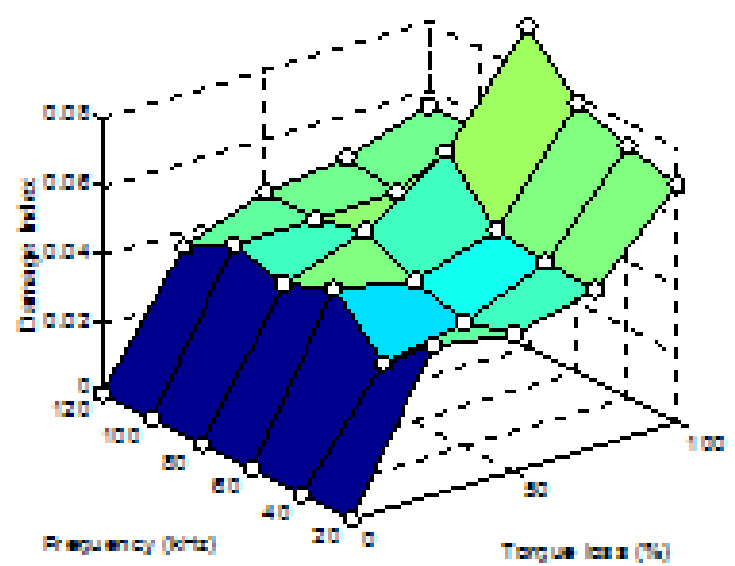

(f) S4 sensor

Figure 3-5-20. Damage index using the energy magnitudes in frequency vs. B0 and B6 torque loss and excitation frequency, E2 excitation. 


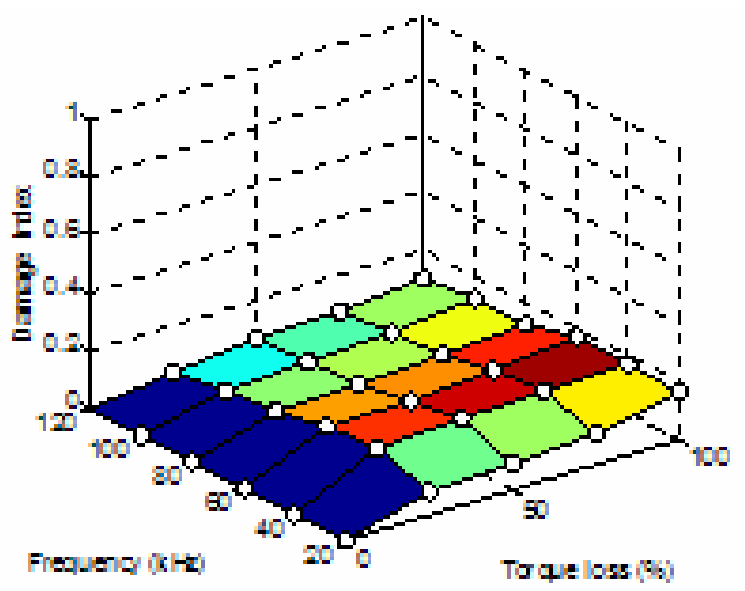

(a) $\mathrm{S} 1$ sensor

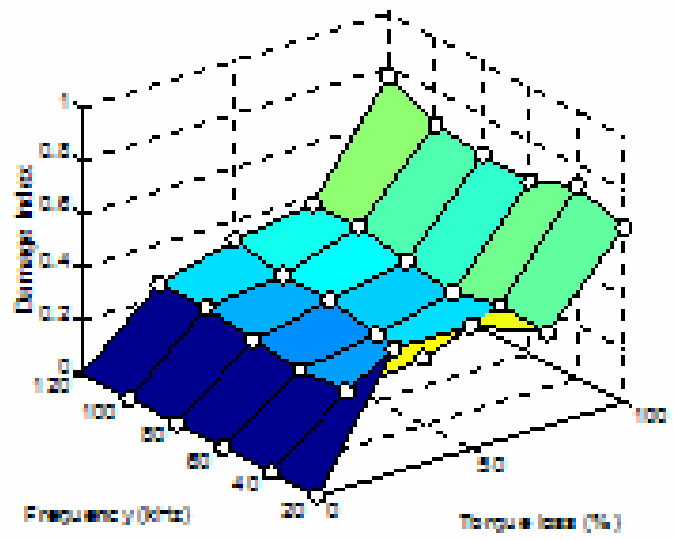

(b) $\mathrm{S} 3$ sensor

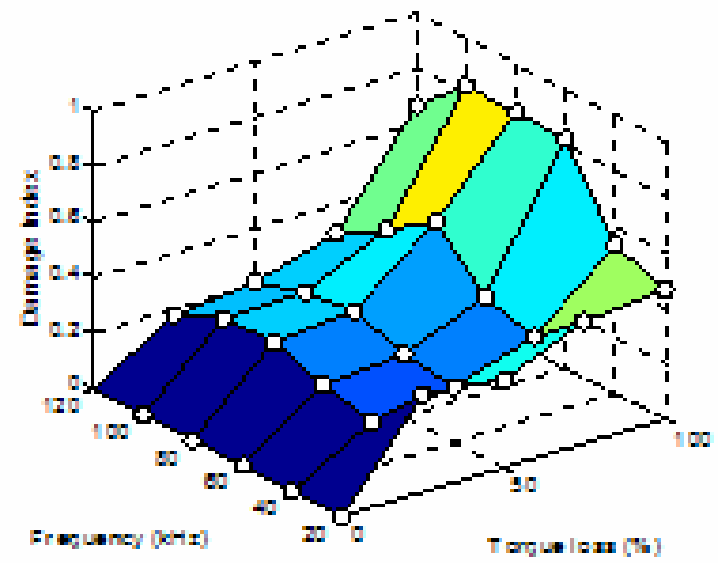

(c) $\mathrm{S} 4$ sensor

Figure 3-5-21. Damage index using the signals in time vs. B0 and B6 torque loss and excitation frequency, E2 excitation. 


\subsubsection{Bolt 0 and Bolt 6, Excitation Case III}

Figure 3-5-22 shows the excitation and sensing configuration and indicates the Bolt 0 and Bolt 6 locations of damage due to torque loss. In this scenario, excitation occurred in another of the quadrants of damage, Quadrant 3, close to Bolt 0. Figure 3-5-23 presents the damage index using the energy magnitudes due to frequency response $\left(D I_{f}\right)$ at $\mathrm{S} 1, \mathrm{~S} 2$ and S4 under E3 excitation. Figure 3-5-24 presents the damage index using the signals in time $\left(D I_{t}\right)$ at $\mathrm{S} 1, \mathrm{~S} 2$ and $\mathrm{S} 4$ sensing locations. As shown previously under the E2 excitation case, quantifiable damage index due to time and frequency responses is shown, indicating favorable damage detection capabilities under a scenario where excitation was present in the same quadrant as damage due to torque loss.

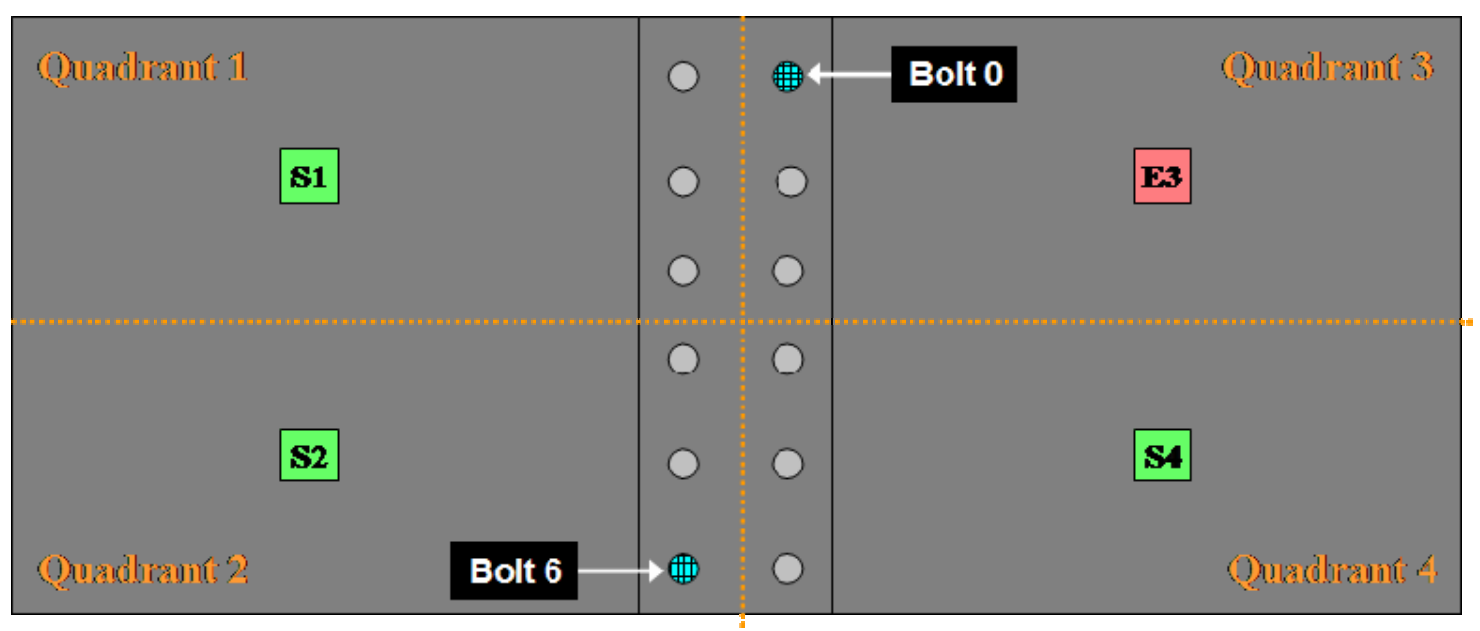

Figure 3-5-22. Excitation Case III, dual Bolt 0 and Bolt 6 variable torque loss. 


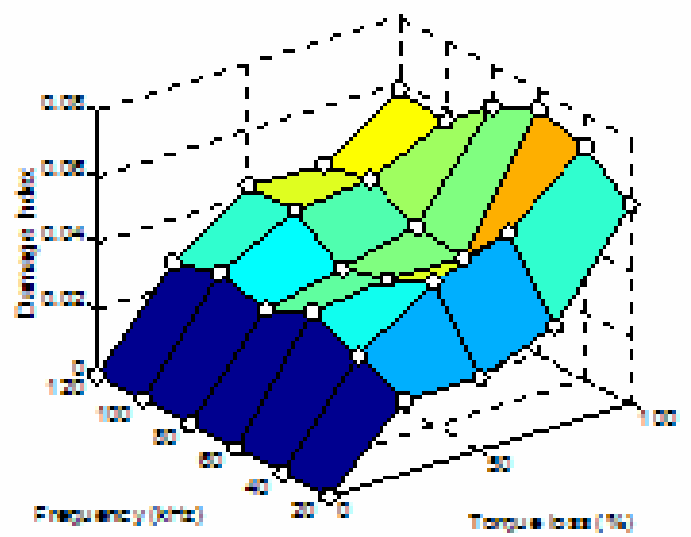

(a) $\mathrm{Sl}$ sensor

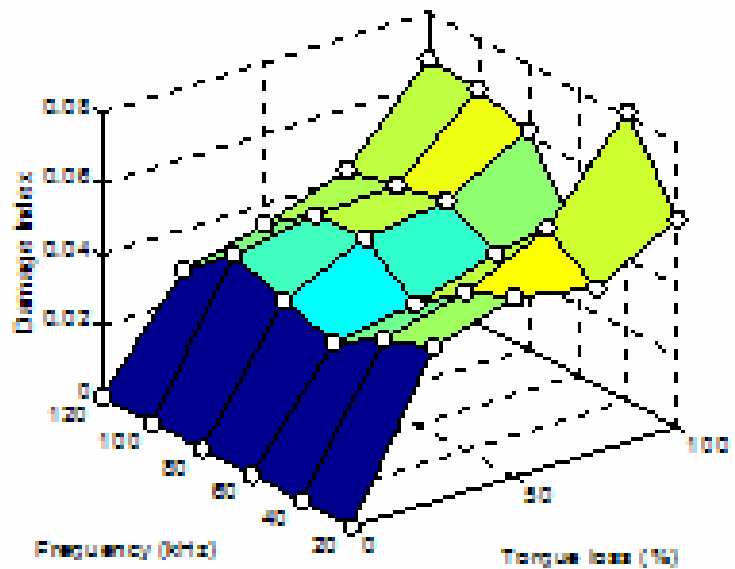

(b) $\mathrm{S2}$ sensor

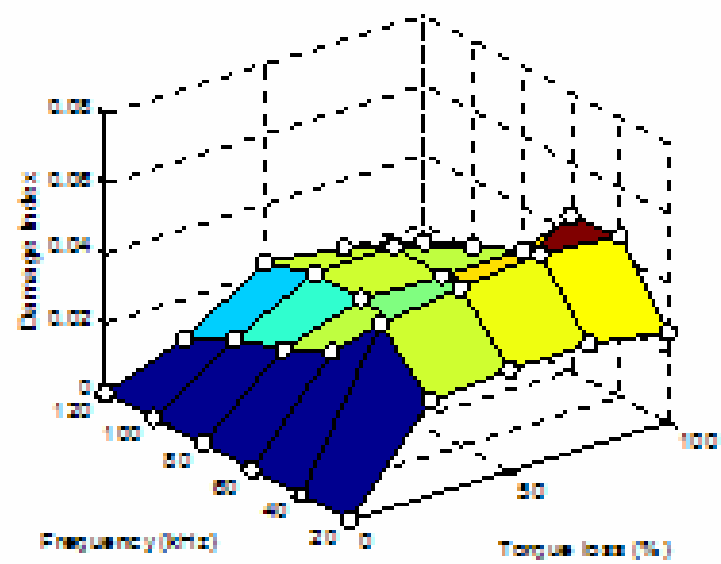

(c) $\mathrm{S} 4$ sensor

Figure 3-5-23. Damage index using the energy magnitudes in frequency vs. B0 and B6 torque loss and excitation frequency, E3 excitation. 


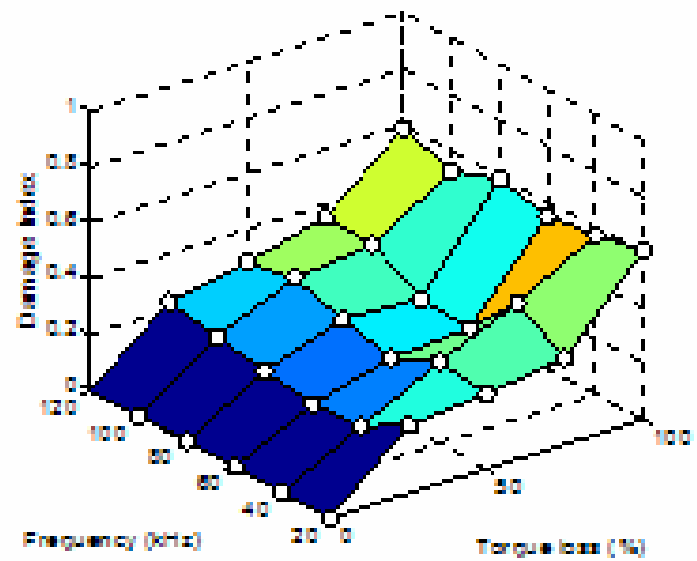

(a) $\mathrm{Sl}$ sensor

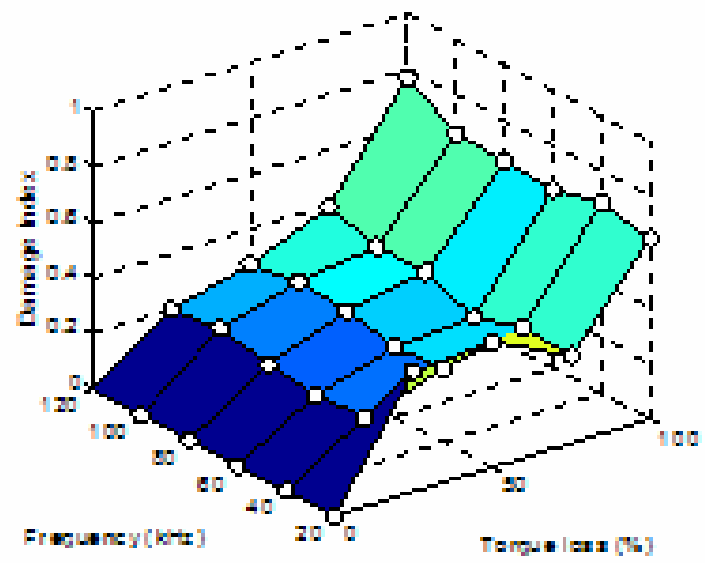

(b) $\mathrm{S} 2$ sensor

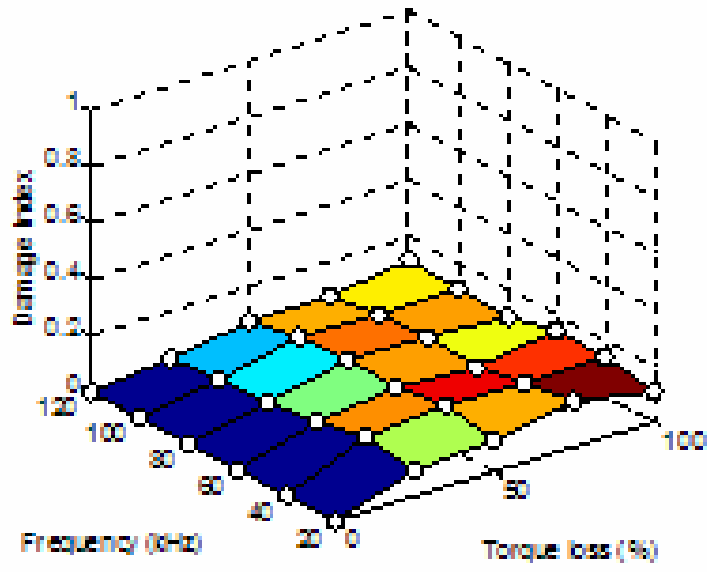

(c) $\mathrm{S} 4$ sensor

Figure 3-5-24. Damage index using the signals in time vs. B0 and B6 torque loss and excitation frequency, E3 excitation. 


\subsubsection{Bolt 0 and Bolt 6, Excitation Case IV}

Figure 3-5-25 shows the excitation and sensing configuration and indicates the Bolt 0 and Bolt 6 locations of damage due to torque loss. Figure 3-5-26 presents the damage index using the energy magnitudes due to frequency response $\left(D I_{f}\right)$ at S1, S2 and S3 under E4 excitation. Figure 43-5-27 presents the damage index using the signals in time $\left(D I_{t}\right)$ at $\mathrm{S} 1, \mathrm{~S} 2$ and $\mathrm{S} 3$ sensing locations. As shown in the three previous excitation cases, there was quantifiable damage index due to time and frequency responses, indicating favorable damage detection capabilities under the fourth excitation configuration case.

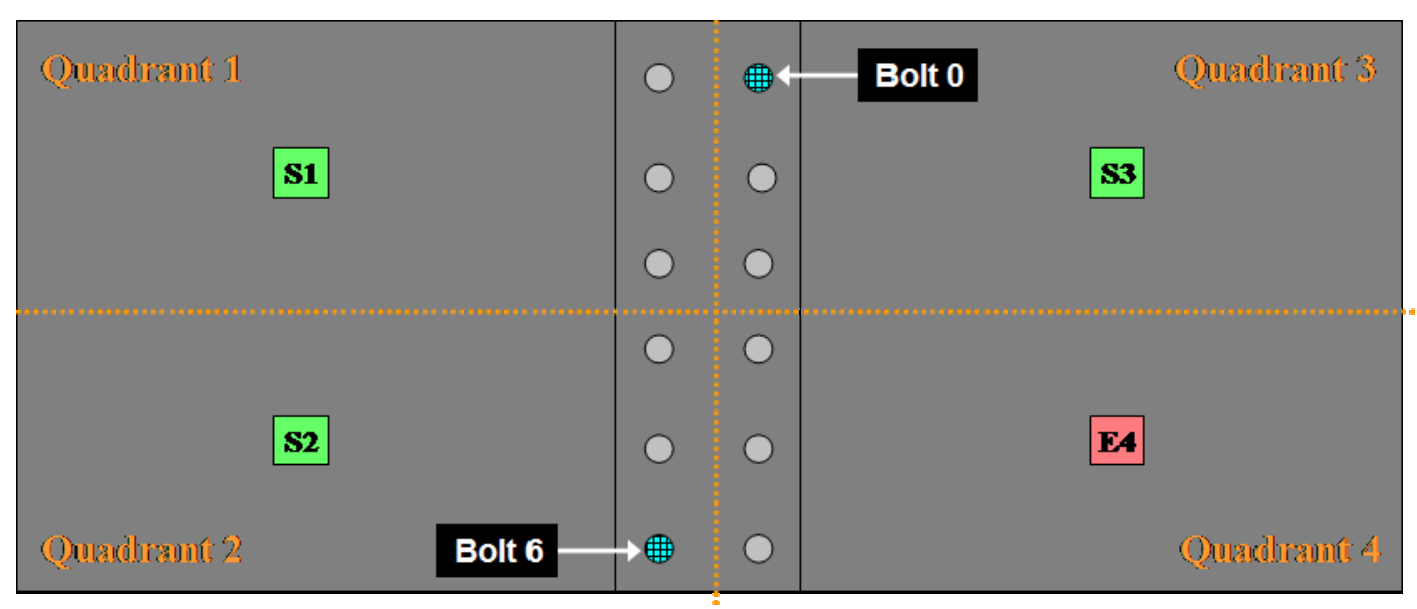

Figure 3-5-25. Excitation Case IV, dual Bolt 0 and Bolt 6 variable torque loss. 


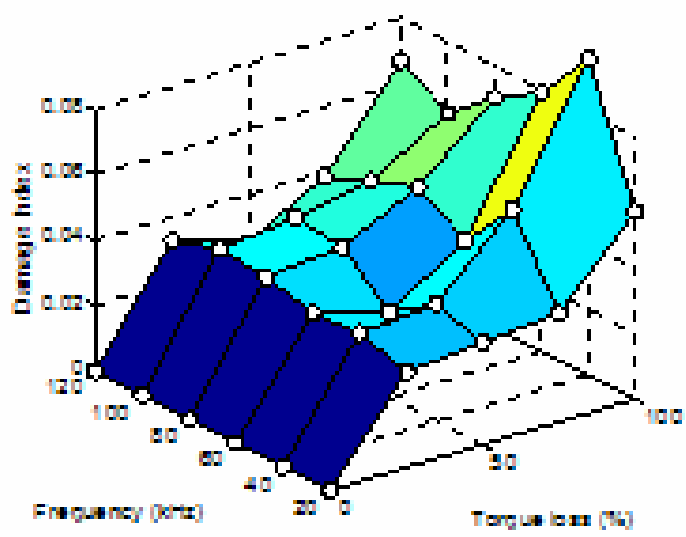

(a) $\mathrm{Sl}$ sensor

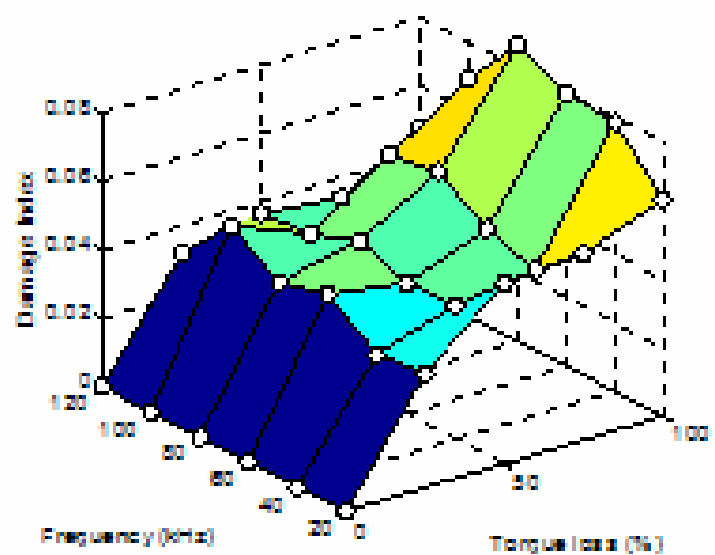

(b) $\mathrm{S} 2$ sensor

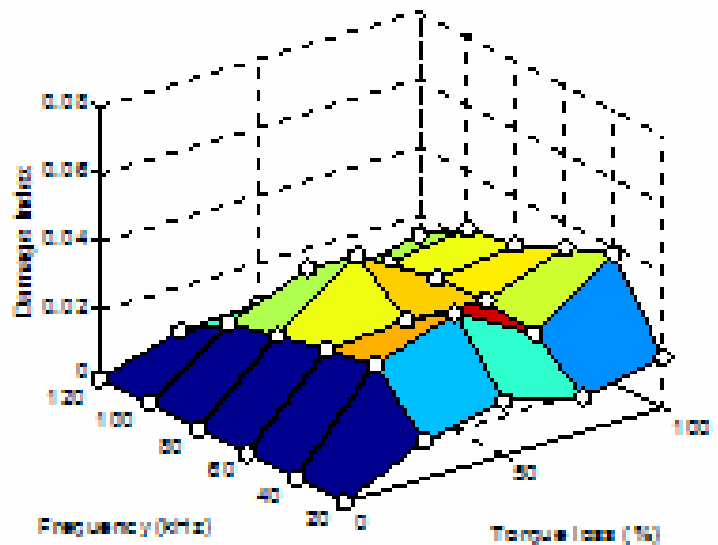

(c) $\mathrm{S} 3$ sensor

Figure 3-5-26. Damage index using the energy magnitudes in frequency vs. B0 and B6 torque loss and excitation frequency, E4 excitation. 


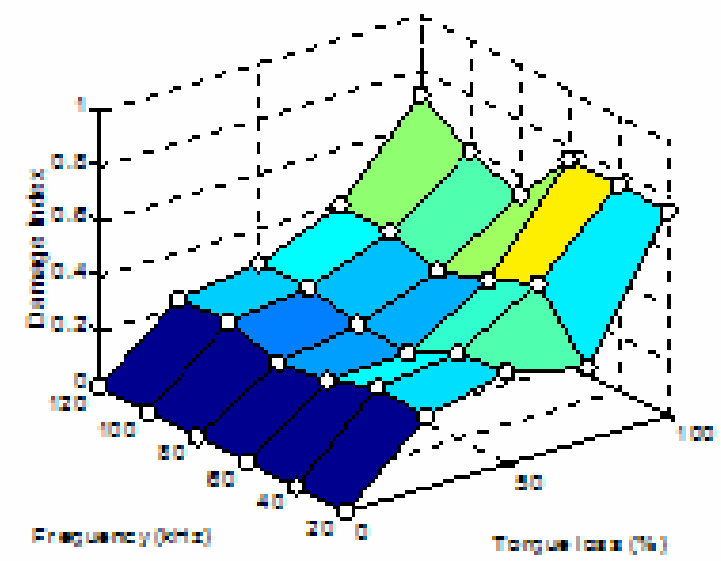

(a) $\mathrm{Sl}$ sensor

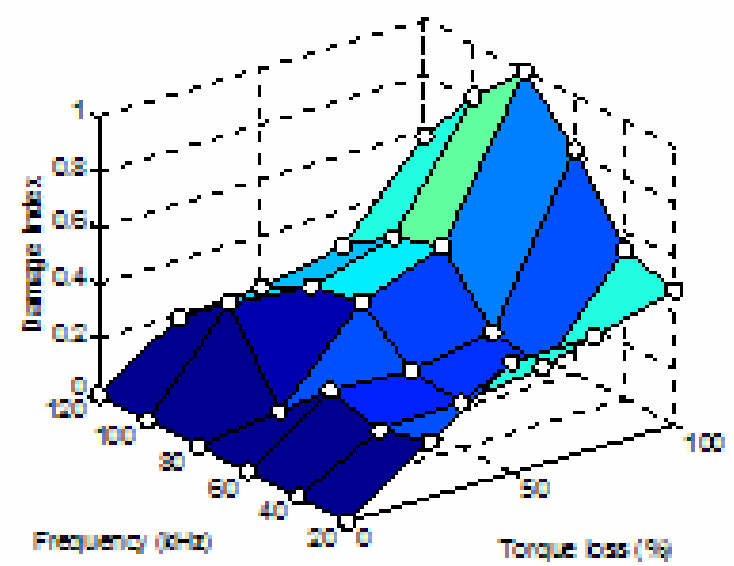

(b) $\mathrm{S} 2$ sensor

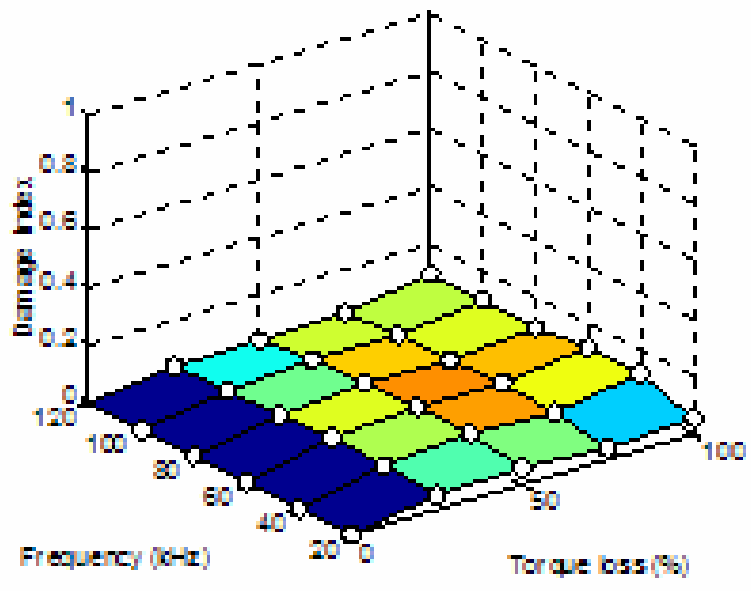

(c) $\mathrm{S3}$ sensor

Figure 3-5-27. Damage index using the signals in time vs. B0 and B6 torque loss and excitation frequency, E4 excitation. 


\subsubsection{Comparison of dual Bolt 0 and Bolt 6 excitation configurations}

The investigate the effects of two damaged bolts within two different quadrants, Figure 3-5-28 shows the comparison of the normalized average damage index of the composite jointed panel due to dual-bolt torque loss within Bolt 0 and Bolt 6 for each excitation configuration. The Lamb wave method exhibited favorable sensitivity to damage in all excitation cases, as indicated by the average damage index quantities at all values of torque loss. In each configuration scenario, the damage indices show a higher sensitivity to changes in bolt torque loss and a lower sensitivity to changes in excitation frequency. The ability of the Lamb wave method to localize damage under a dual-bolt damage scenario was examined in the same manner as the single-bolt damage cases. The following analysis was made to provide a rough estimate of the location of damage due to torque loss:

$$
\begin{array}{ll}
D I_{E 1, S 4} \approx D I_{E 1, S 3}>>D I_{E 1, S 2} & \text { Figure 3-5-28 (a) } \\
D I_{E 2, S 4} \approx D I_{E 2, S 3}>>D I_{E 2, S 1} & \text { Figure 3-5-28 (b) } \\
D I_{E 3, S 2} \approx D I_{E 3, S 1}>>D I_{E 3, S 4} & \text { Figure 3-5-28 (c) } \\
D I_{E 4, S 2} \approx D I_{E 4, S 1}>>D I_{E 4, S 3} & \text { Figure 3-5-28 (d) }
\end{array}
$$

These relationships imply that the damage was located in the common area between sensing locations S2 and S4 and also within the area between sensing locations S1 and S3. From this information alone, all quadrants were likely candidates for damage due to bolt torque loss. 


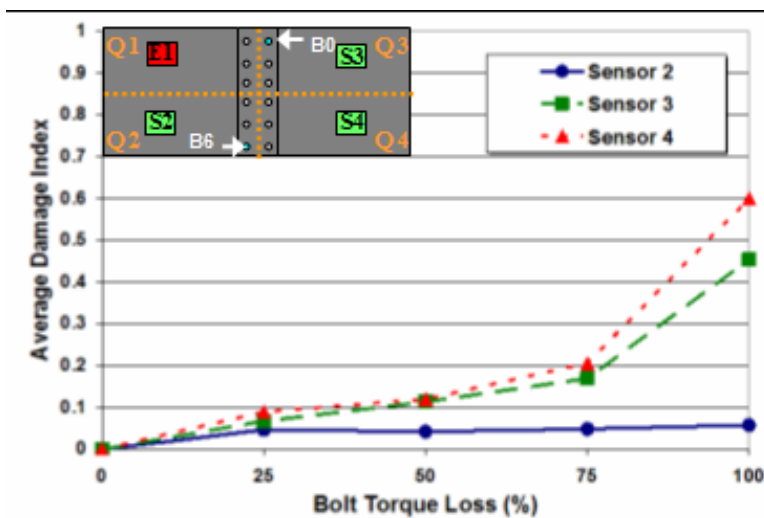

(a) E1 excitation

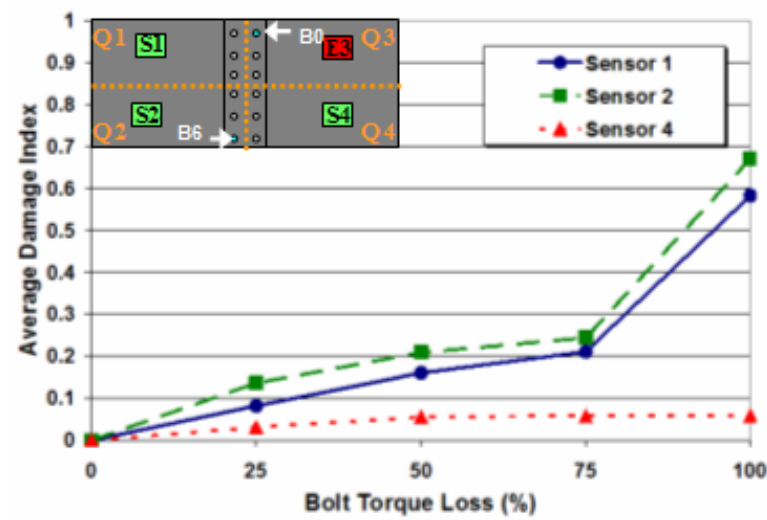

(c) E3 excitation

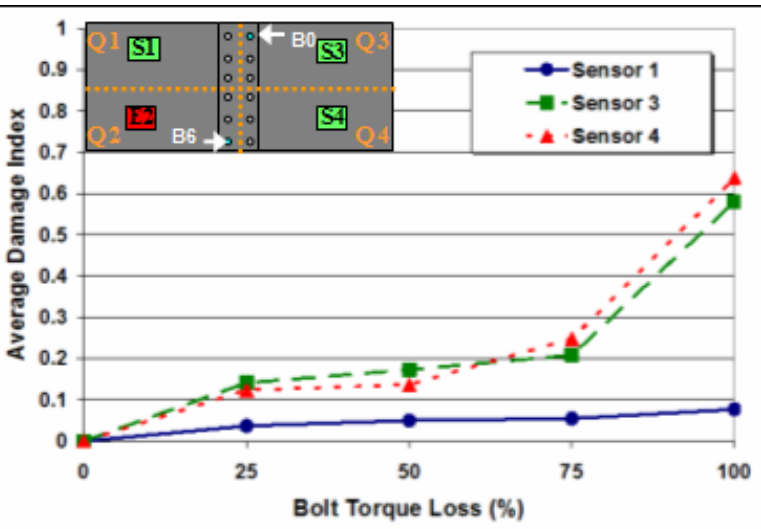

(b) E2 excitation

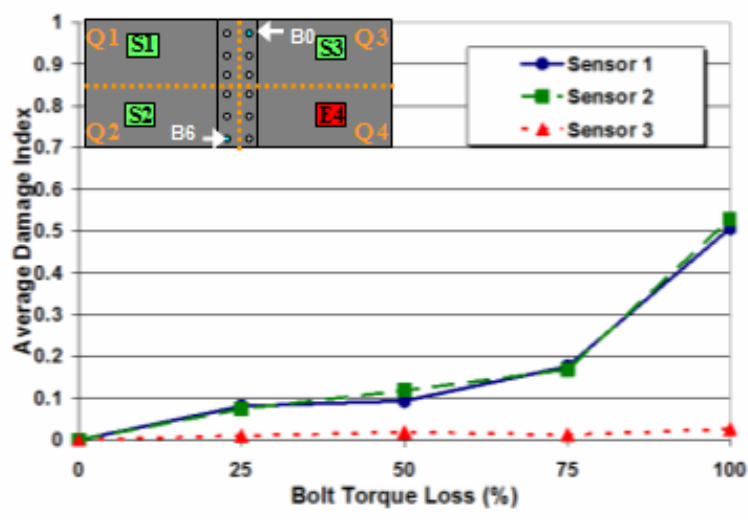

(d) E4 excitation

Figure 3-5-28. Comparison of normalized average damage index, Bolt 0 and Bolt 6 dualbolt torque loss.

To discriminate between Quadrants 1 and 3, these trends were compared in a similar manner to single-bolt damage scenarios in Quadrant 3. The isolation and comparison between the information in Figure 3-5-28 (b) and (d) was performed using the sensing information at $\mathrm{S} 3$ and $\mathrm{S} 1$, respectively. Comparing $D I_{E 4, S 1}$ and $D I_{E 2, S 3}$ from Figure 3-5-28 (b) and Figure 3-5-28 (d) clearly shows:

$$
D I_{E 2, S 3}>D I_{E 4, S 1}
$$

The information is isolated to show this comparison graphically in Figure 3-5-29. 


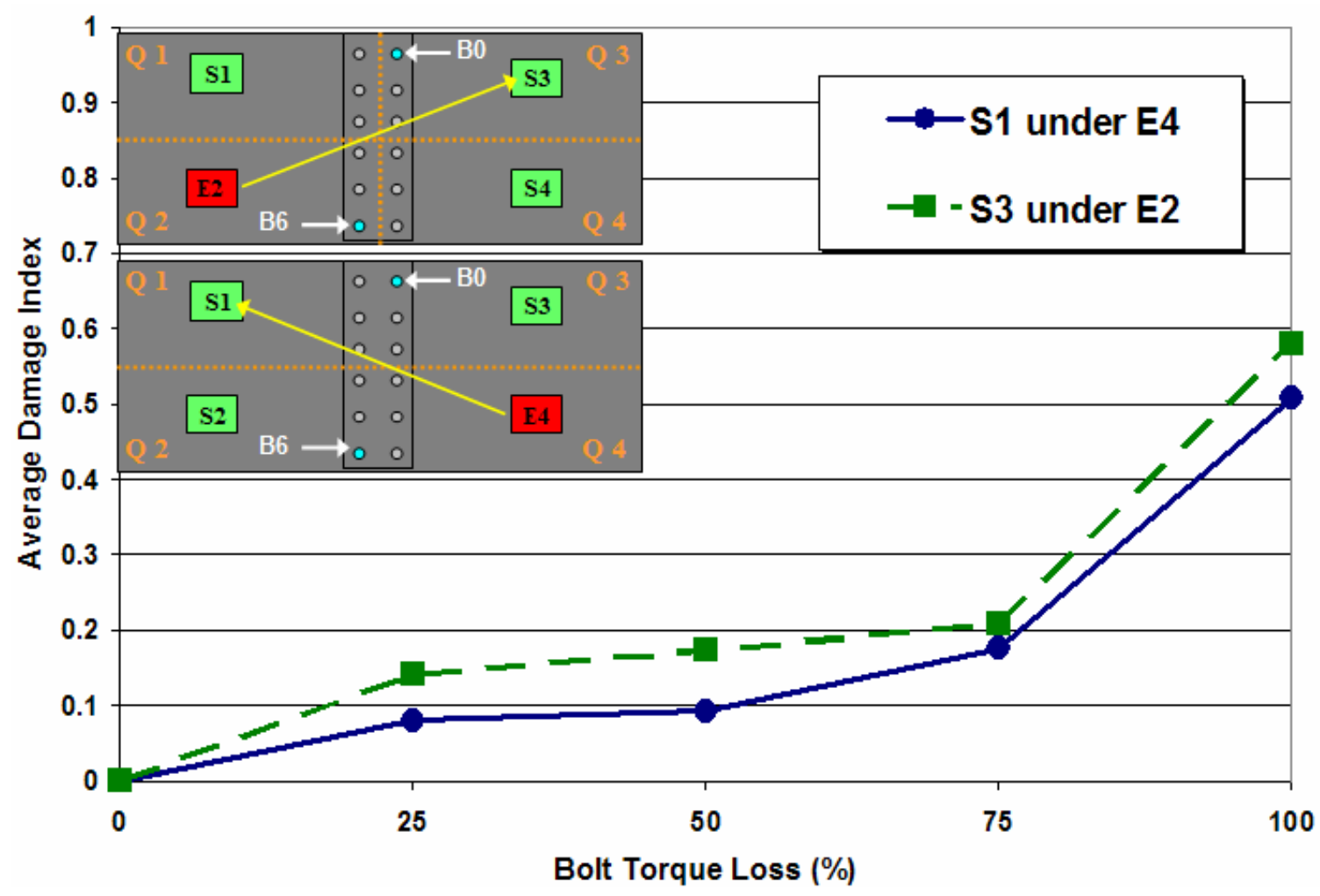

Figure 3-5-29. Comparison of normalized average damage indices, Bolt 0 and Bolt 6 torque loss; $D I_{E 4, S l}$ and $D I_{E 2, S 3}$.

It is necessary to perform a second comparison in an effort to distinguish between damage within Quadrant 2 and Quadrant 4. This analysis is performed in a similar manner to Bolt 6 information in section 3.4.5. A method for distinction between data for possible damage located in-between sensors S2 and S4 comes from the E1 and E3 excitation cases. Figure 3-5-30 shows the comparison of $D I_{E 3, S 2}$ and $D I_{E l, S 4}$ from Figure 3-5-28 (a) and Figure 3-5-28 (c). The information is isolated in Figure 3-5-30 and clearly shows:

$$
D I_{E 3, S 2}>D I_{E 1, S 4}
$$


This indicates damage was likely within Quadrant 2 as compared to Quadrant 4 for the second damaged bolt due to torque loss.

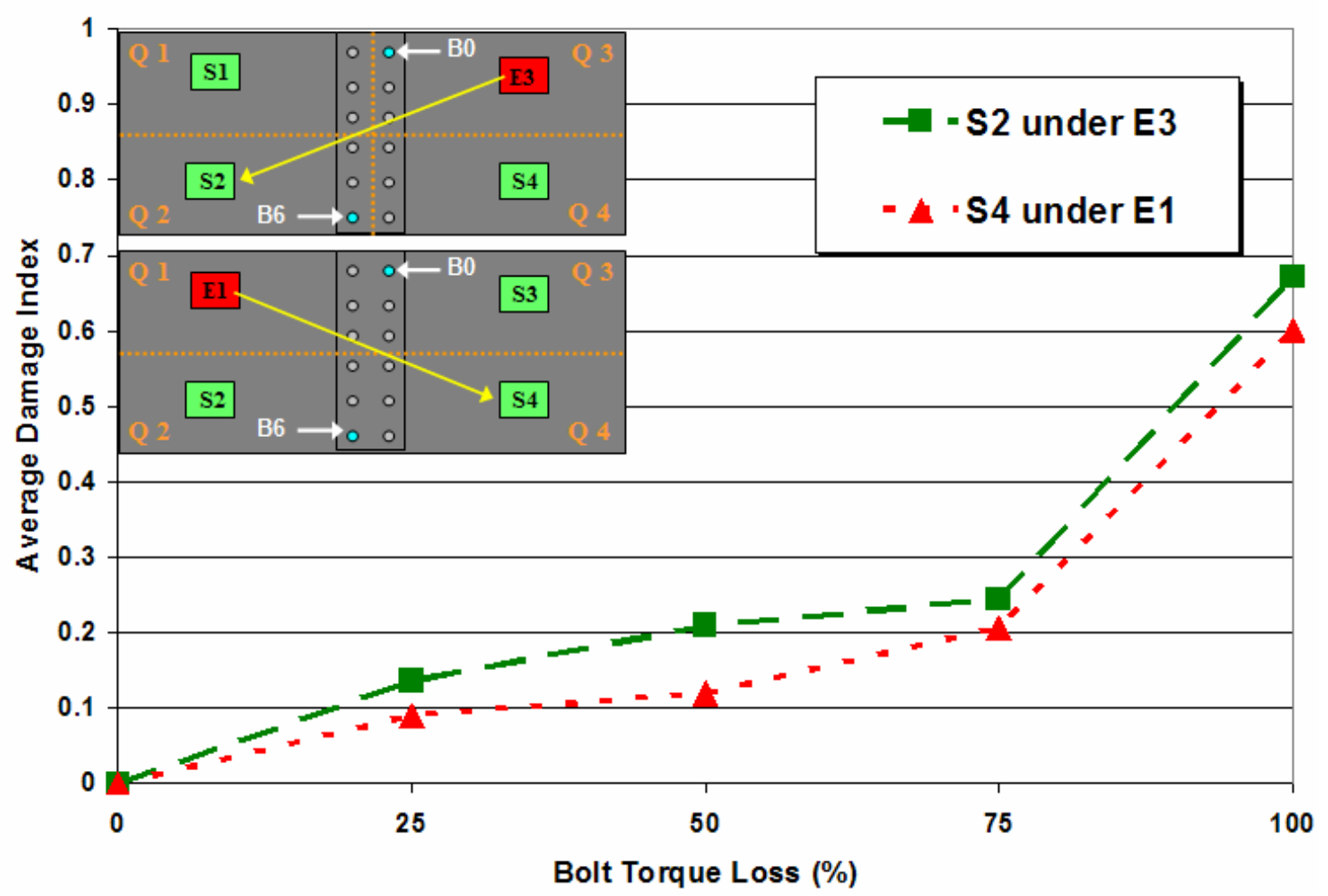

Figure 3-5-30. Comparison of normalized average damage indices, Bolt 0 and Bolt 6 torque loss; $D I_{E 3, S 2}$ and $D I_{E 1, S 4}$.

The average damage index values under this dual-bolt damage scenario are capable of providing insight into the detection and localization of damage due to bolt torque loss within the composite joint. Combining the two relationships indicates a dualdamage scenario present in Quadrant 3 and Quadrant 2. Figure 3-5-31 shows the two shaded areas of the highest likelihood of damage. 


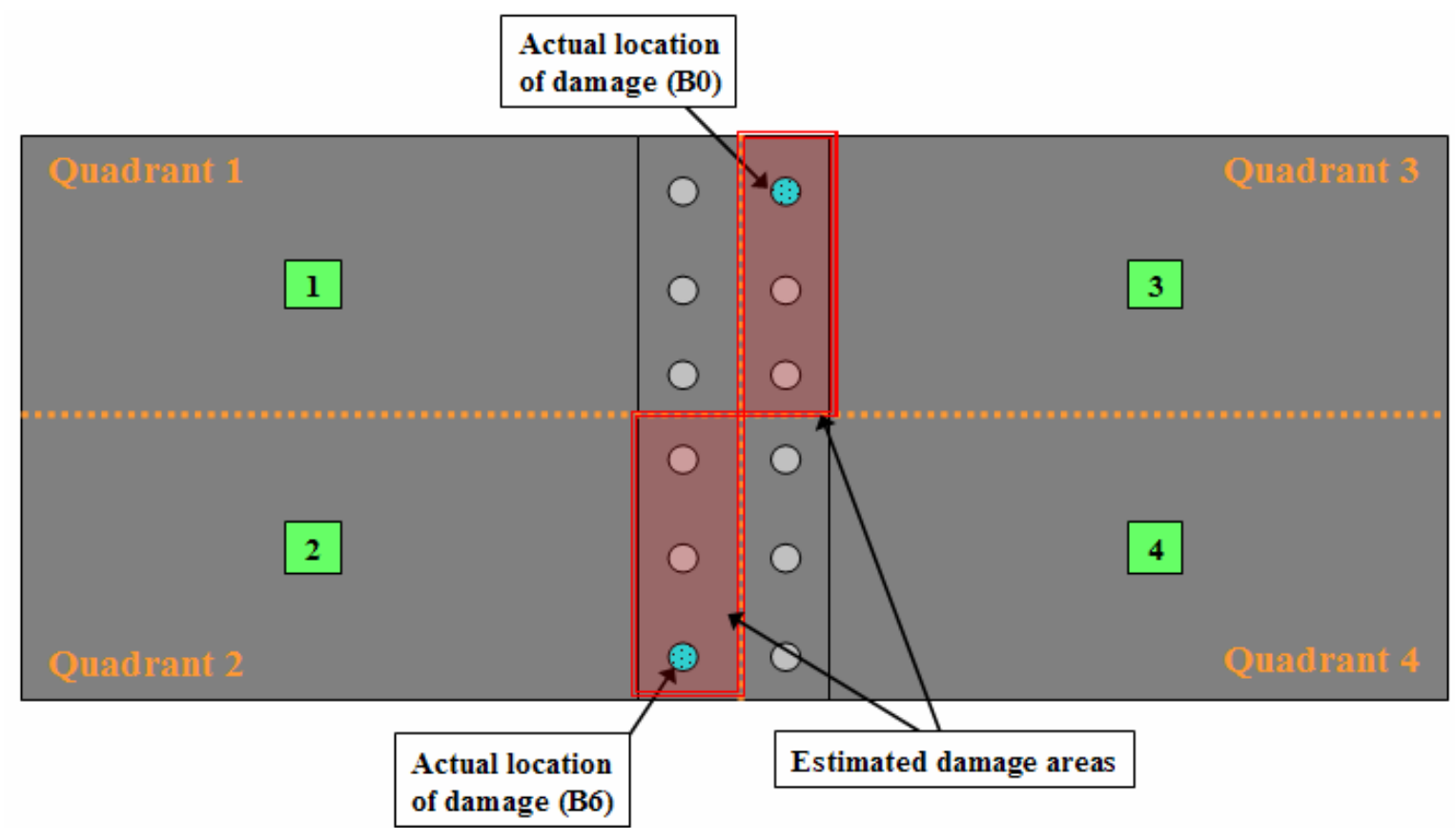

Figure 3-5-31. Estimated damage area due to Bolt 0 and Bolt 1 torque loss.

\subsection{Sensing Variation of Undamaged Composite Jointed Panel over Time}

The normalized voltage response of sensors S1, S3 and S4 at $40 \mathrm{kHz}$ under E2 excitation was compiled during the period of experimentation spanning from October 2007 to February 2008. Figure 3-6-1 shows the normalized time response of each sensor over the time-span of experimentation. 


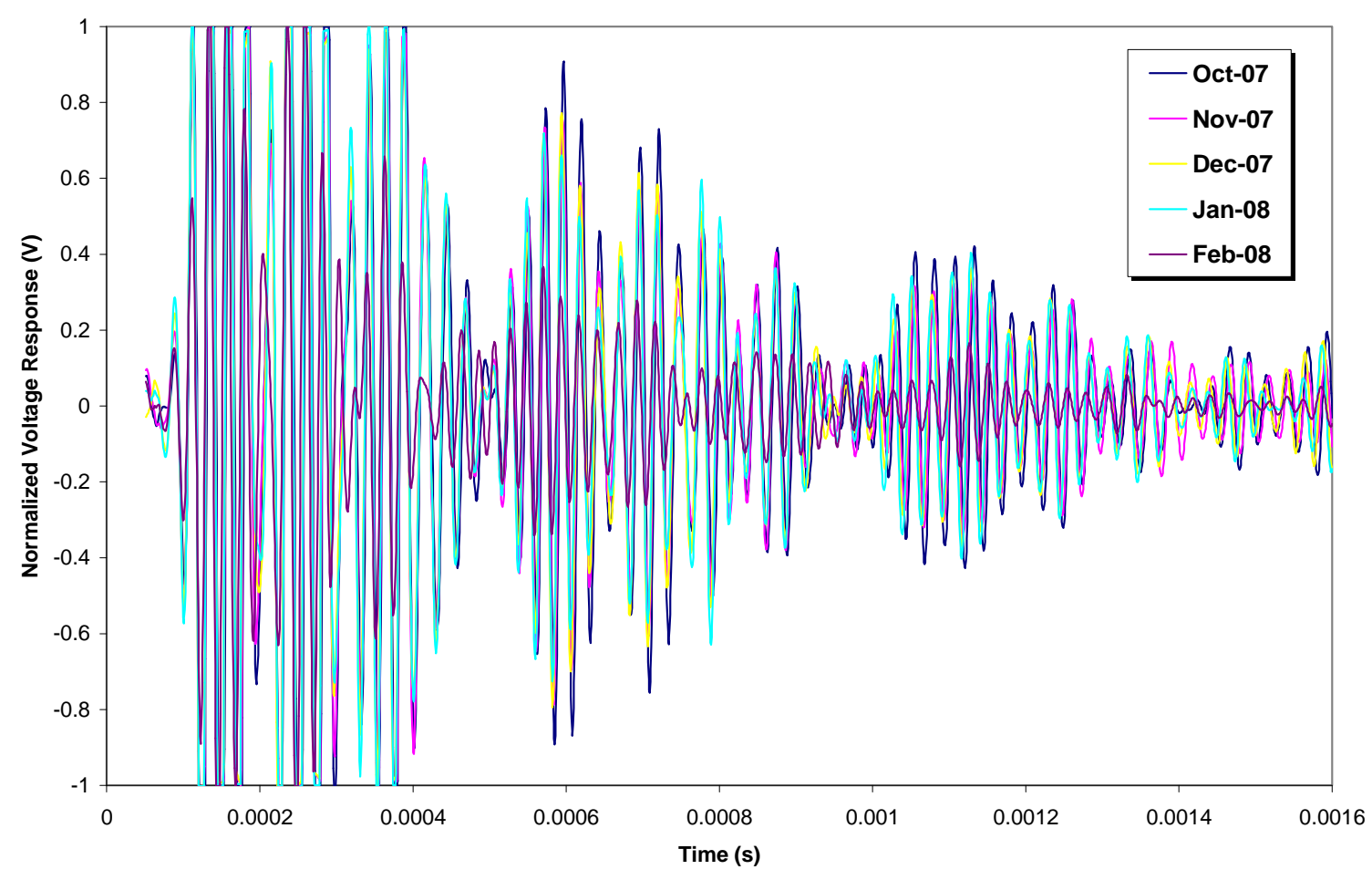

(a) S1 signal

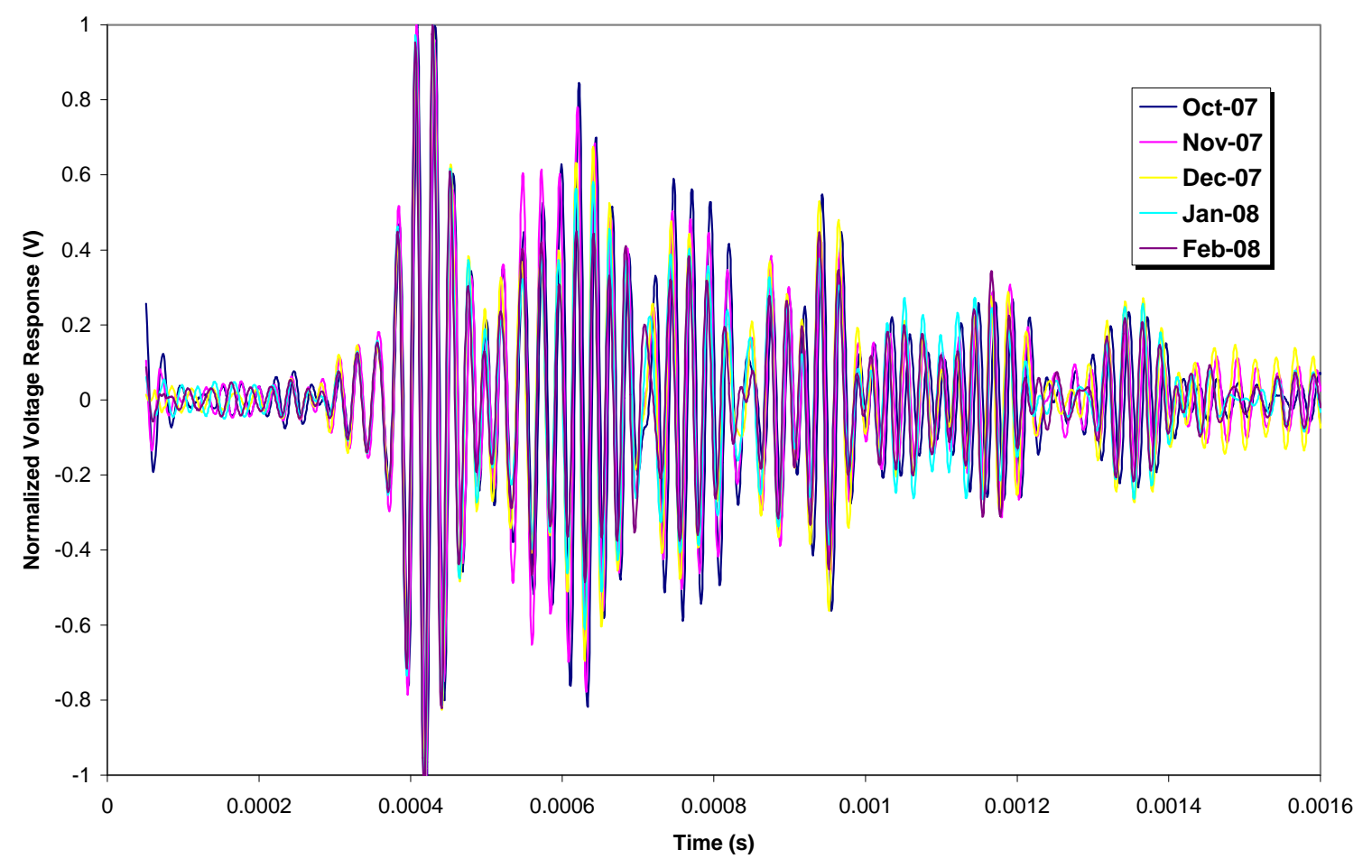

(b) S3 signal 


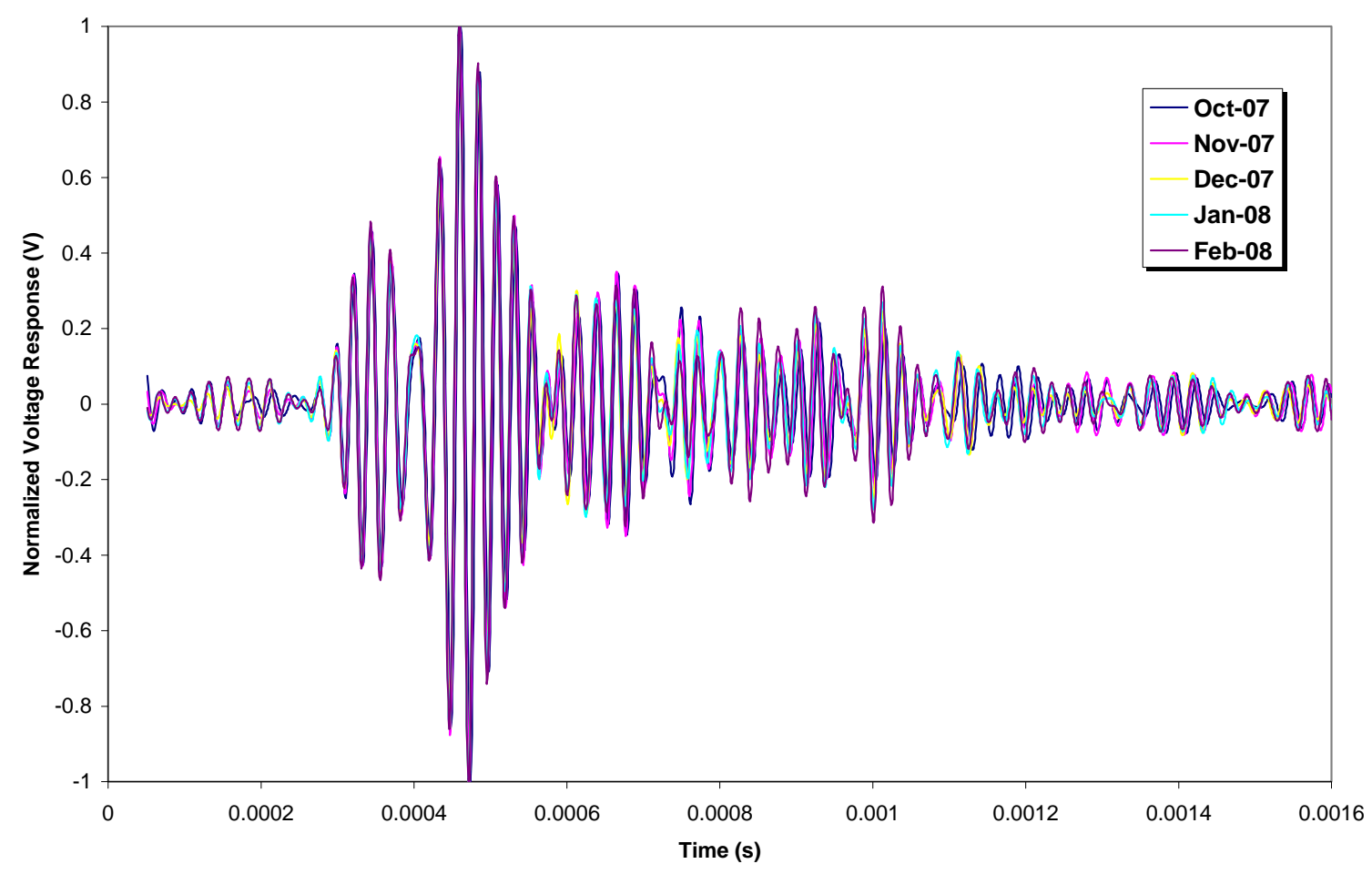

(c) S4 signal

Figure 3-6-1. Normalized baseline time response over five months, E2 $40 \mathrm{kHz}$ excitation.

The voltage response plots nearly coincide for each month of experimentation, demonstrating a slight but noticeable change in undamaged (baseline) time-response information over the duration of experimentation on the composite jointed panel. At each sensor, the plots deviate from one another within 3\%. To further investigate the timeresponse signal deviation over the five month period of experimentation, the maximum time-response from the non-normalized sensing information was compared over all excitation frequencies $(20 \mathrm{kHz}, 40 \mathrm{kHz}, 60 \mathrm{kHz}, 80 \mathrm{kHz}, 100 \mathrm{kHz}$, and $120 \mathrm{kHz})$. Figure 3-6-2 shows the maximum time response values for each of the sensors under the E2 excitation configuration and over a range of excitation frequencies. 


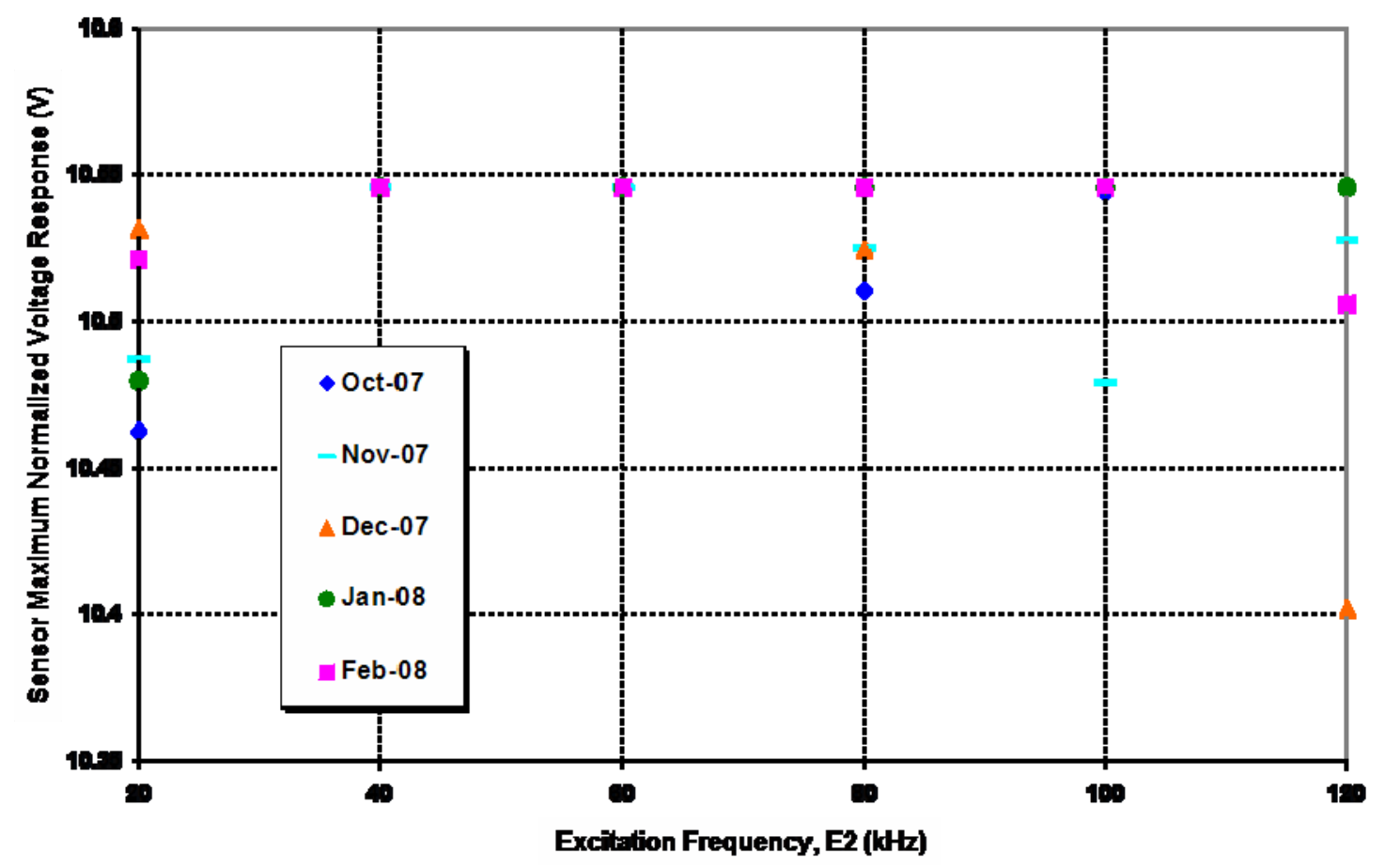

(a) S1 signal

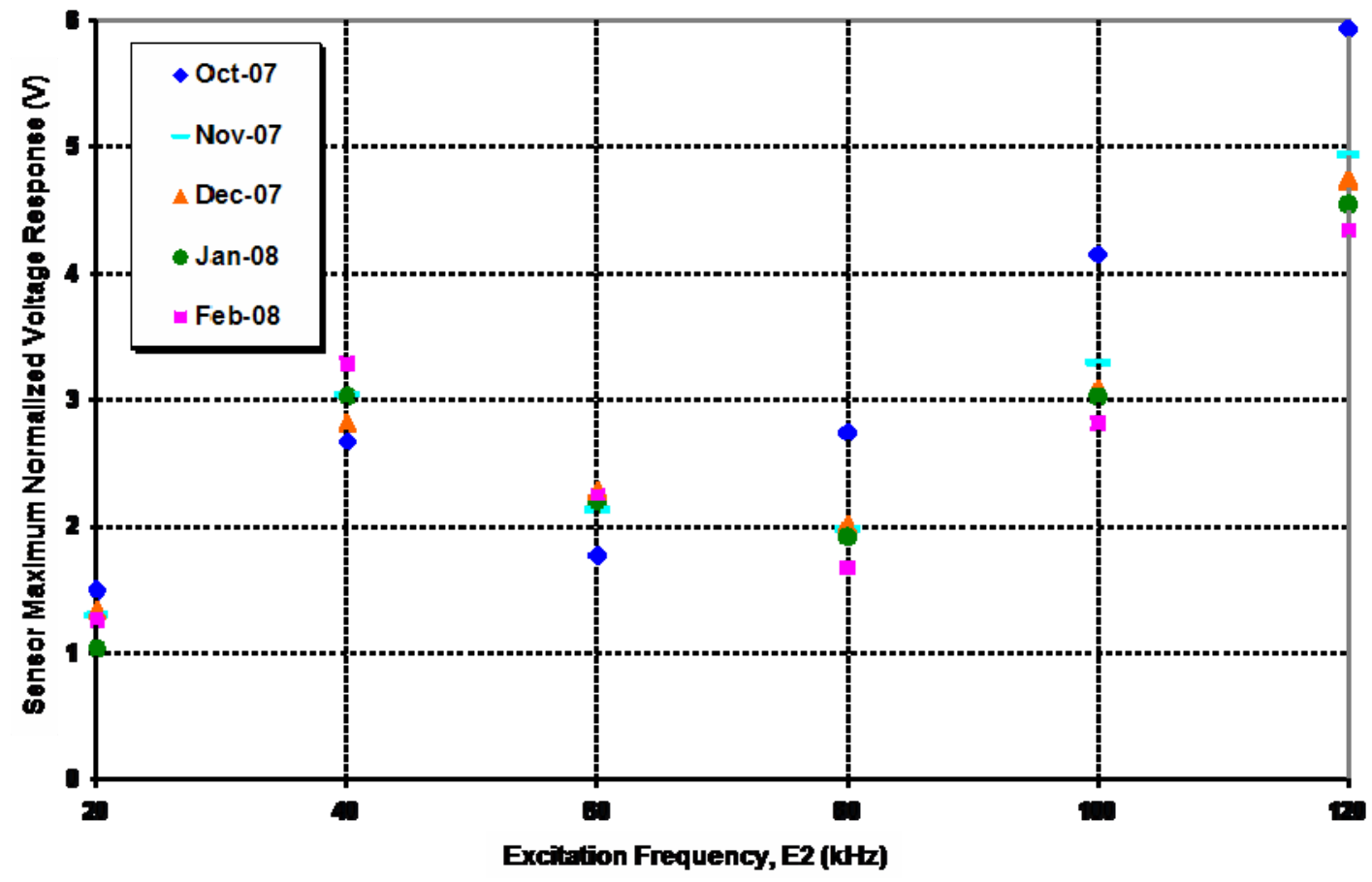

(b) S3 signal 


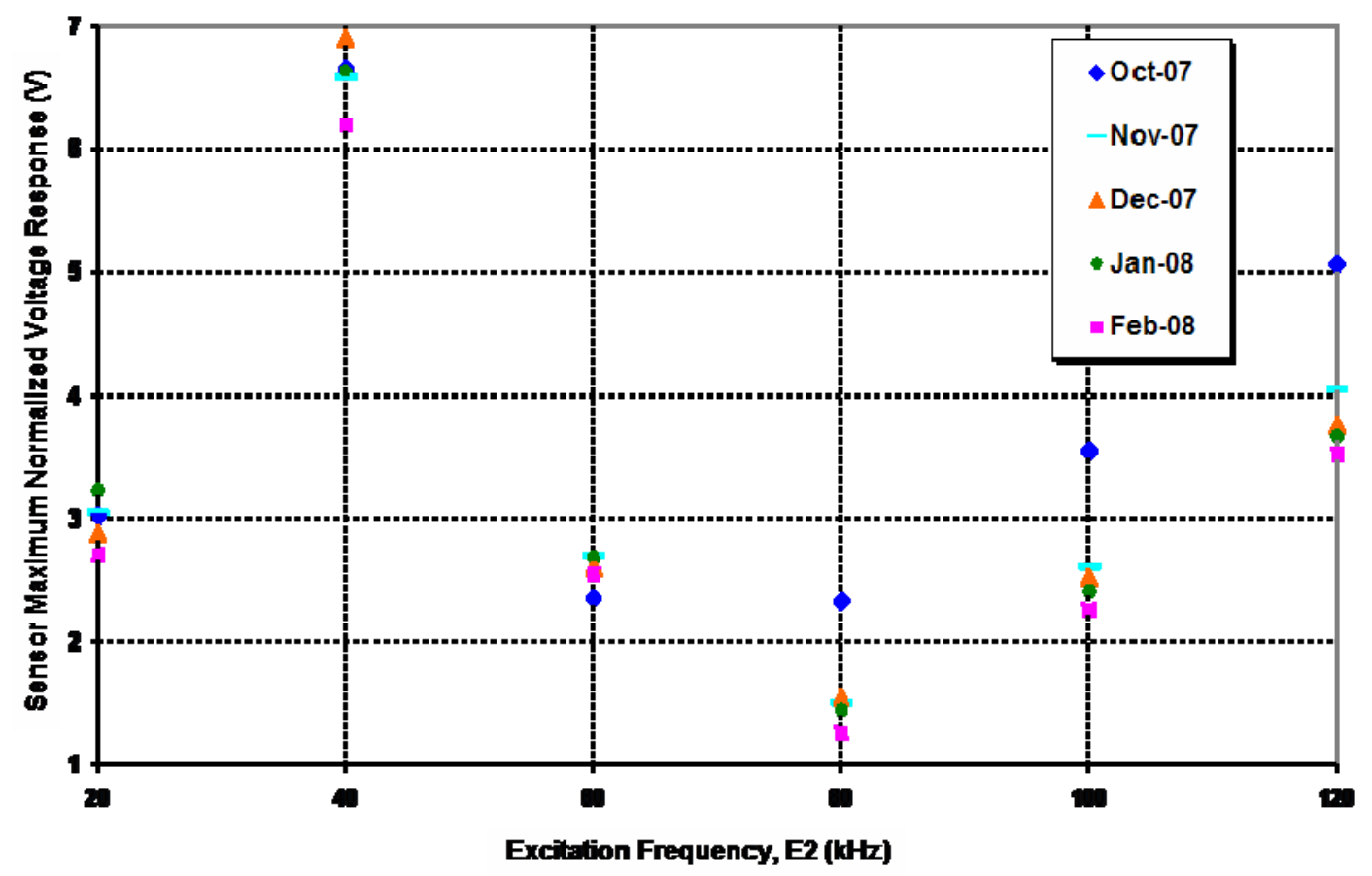

(c) S4 signal

Figure 3-6-2. Normalized baseline voltage response vs. E2 excitation frequency over five months.

The average difference between time-response signals over a range of excitation frequencies and at three sensing locations under an E2 excitation configuration is $4 \%$. Since the damage index relies on an undamaged (baseline) set of time-response information to assess the damage level in the composite jointed panel, it was determined that while the change in sensing response over five months was slight, the fluctuations may have an impact on overall damage index. Using the information from Figure 3-6-1, an average normalized time response for each sensor location was calculated to form an average baseline time-response data set at $40 \mathrm{kHz}$ excitation frequency under E2 excitation configuration. Figure 3-6-3 displays the average normalized baseline plots at each of the three sensing locations. 


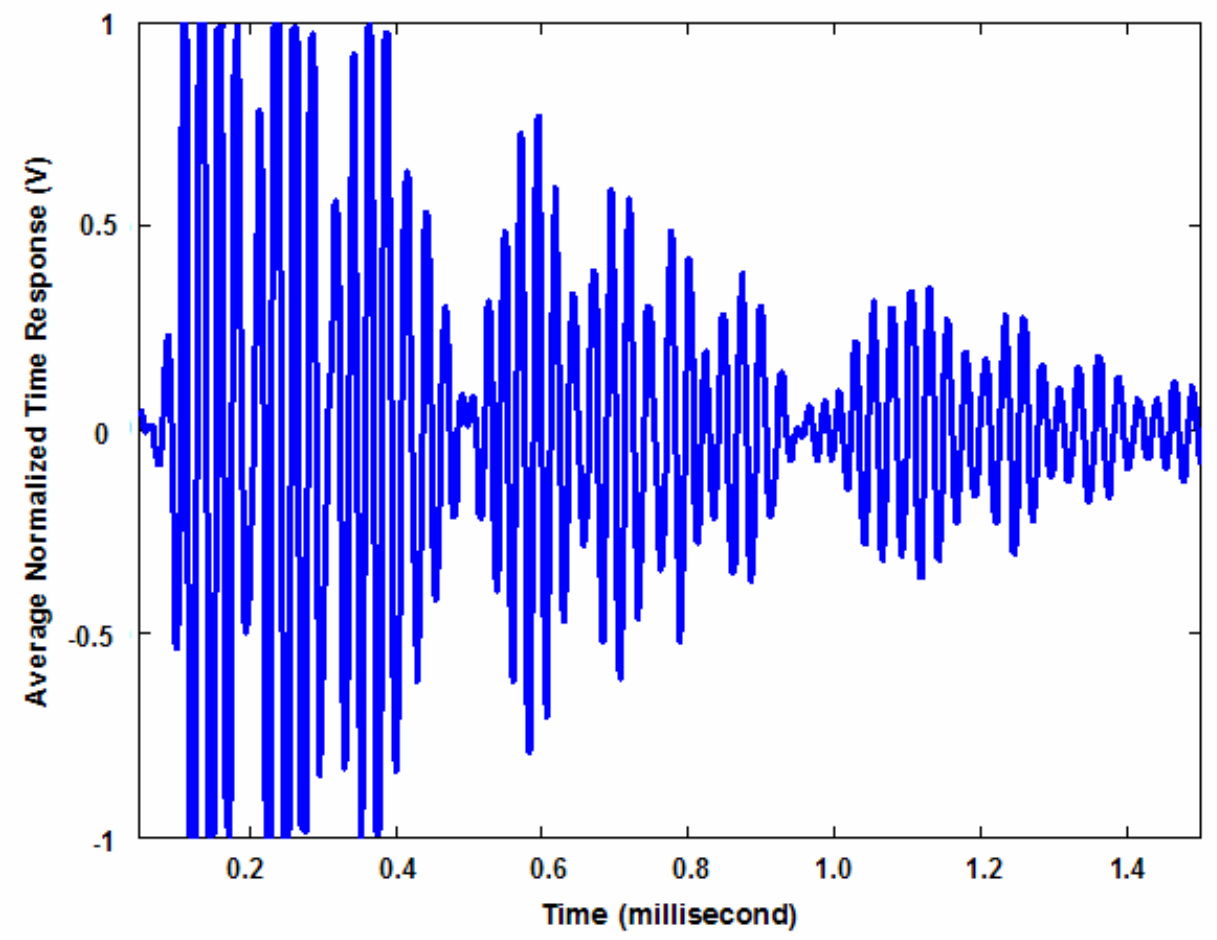

(a) S1 signal

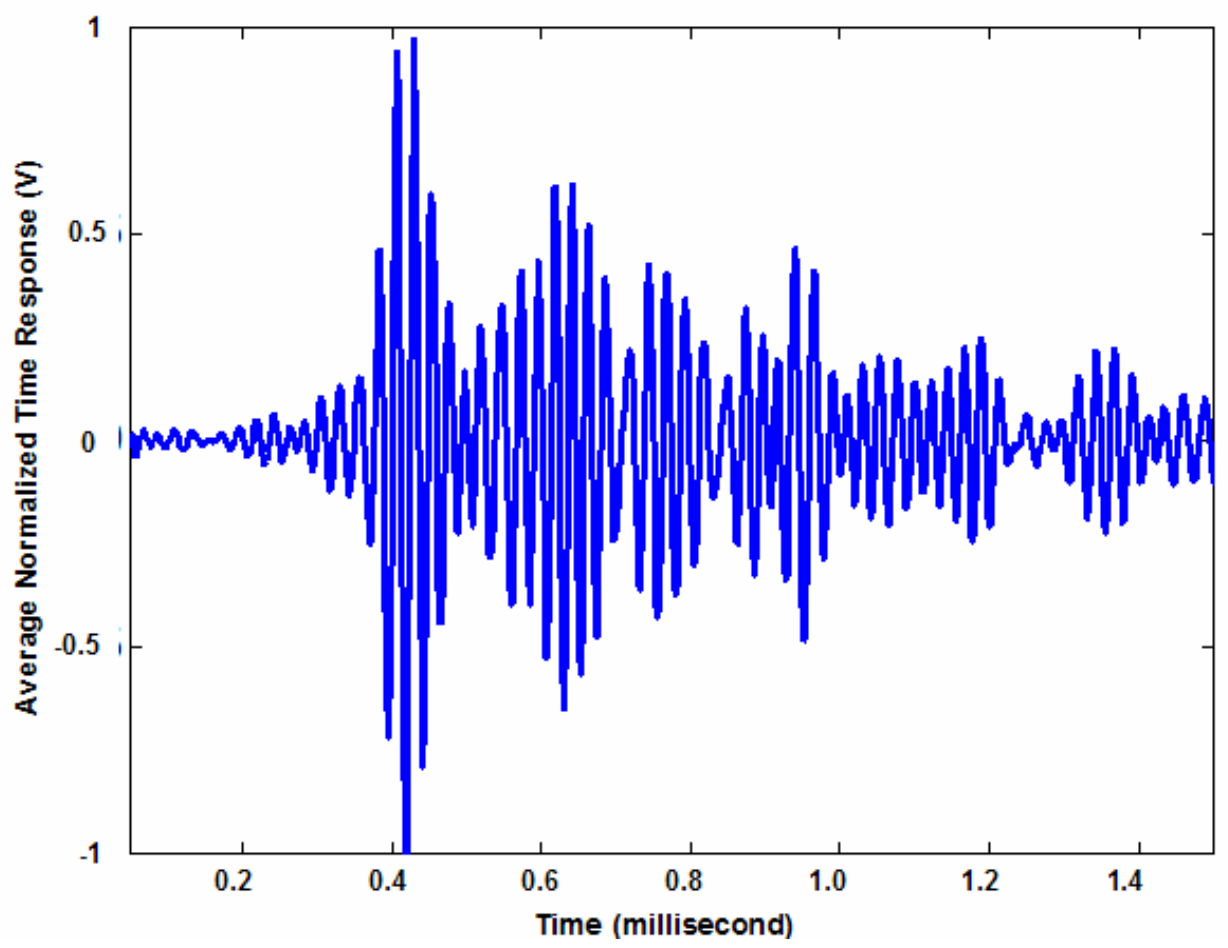

(b) S3 signal 


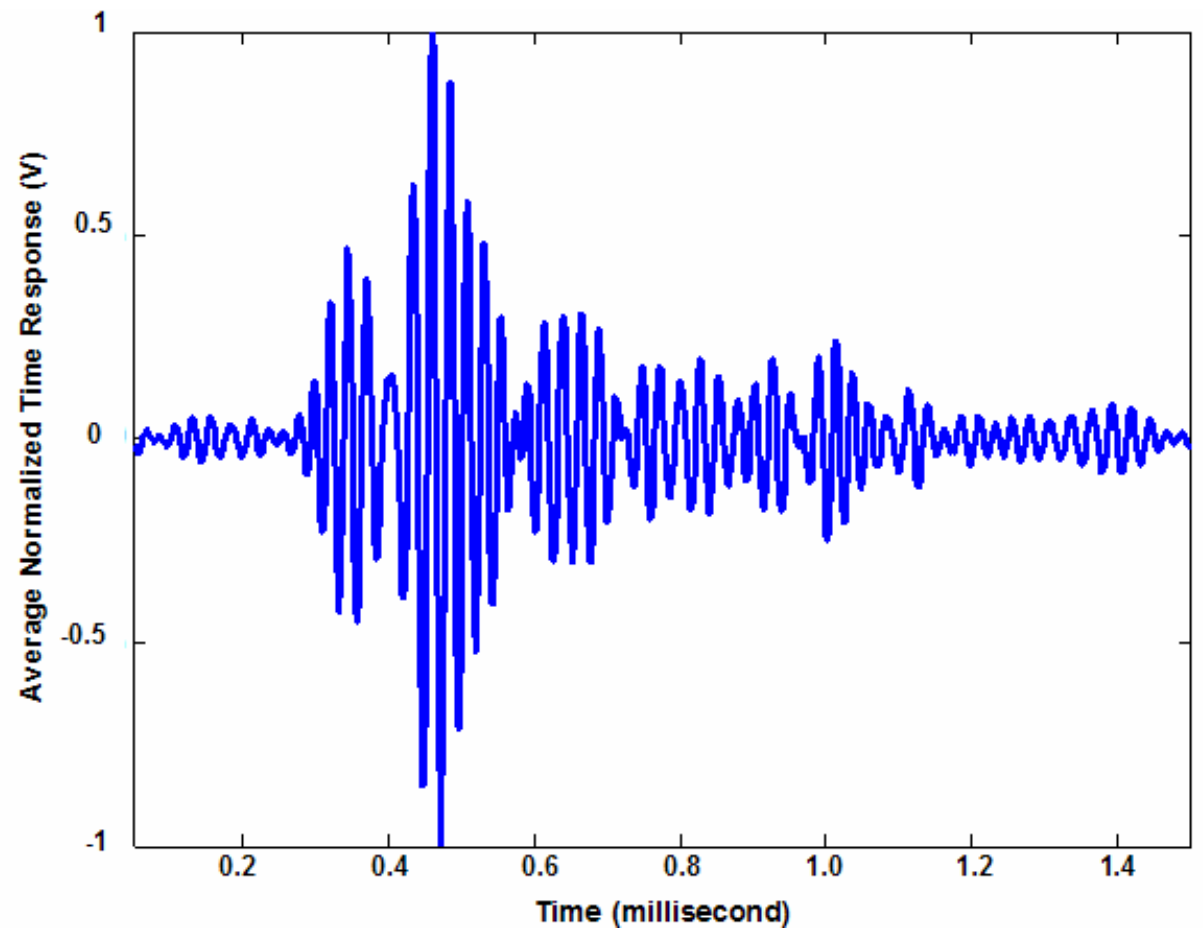

(c) S4 signal

Figure 3-6-3. Average baseline time response, E2 $40 \mathrm{kHz}$ excitation.

\subsection{Sensing Variation due to Temperature Increase of an Active Sensor}

In this chapter, the sensitivity of a piezo-patch to temperature was evaluated to determine the effects on sensing capability. This experimentation was conducted by changing the temperature of the S1 sensor under the E2 excitation case, as explained in Figure 2-7-1. The temperature of S1 was increased beyond ambient temperature $\left(21.7^{\circ} \mathrm{C}\right)$ to $30,40,50,60,70,80^{\circ} \mathrm{C}$. Figure $3-7-1$ presents the temperature sensitivity of the piezopatch sensor by analyzing the maximum time-response. As seen in this figure, at relatively lower excitation frequencies such as $20 \mathrm{kHz}$ and $40 \mathrm{kHz}$, the sensing signal increases with temperature. After $60^{\circ} \mathrm{C}$, the sensing signal decreases as the temperature increases above ambient. For higher excitation frequencies, the sensing voltage 
monotonically decreases as the temperature increases. This phenomenon is more clearly observed in the normalized voltage response, shown in Figure 3-7-2. As the excitation frequency increases, the sensing voltage becomes more strongly dependent on the piezopatch sensor temperature. Under operations which experience a large temperature variance at sensing locations, it would be necessary to compensate for this demonstrated temperature sensitivity of sensors. For the purposes of this research, the temperature conditions under which the composite jointed panel was tested did not experience a shift of more than positive or negative $5^{\circ} \mathrm{C}$. It was deemed unnecessary to compensate the sensor signal response in the damage index calculations under such a slight change in laboratory temperature over the months of experimentation.

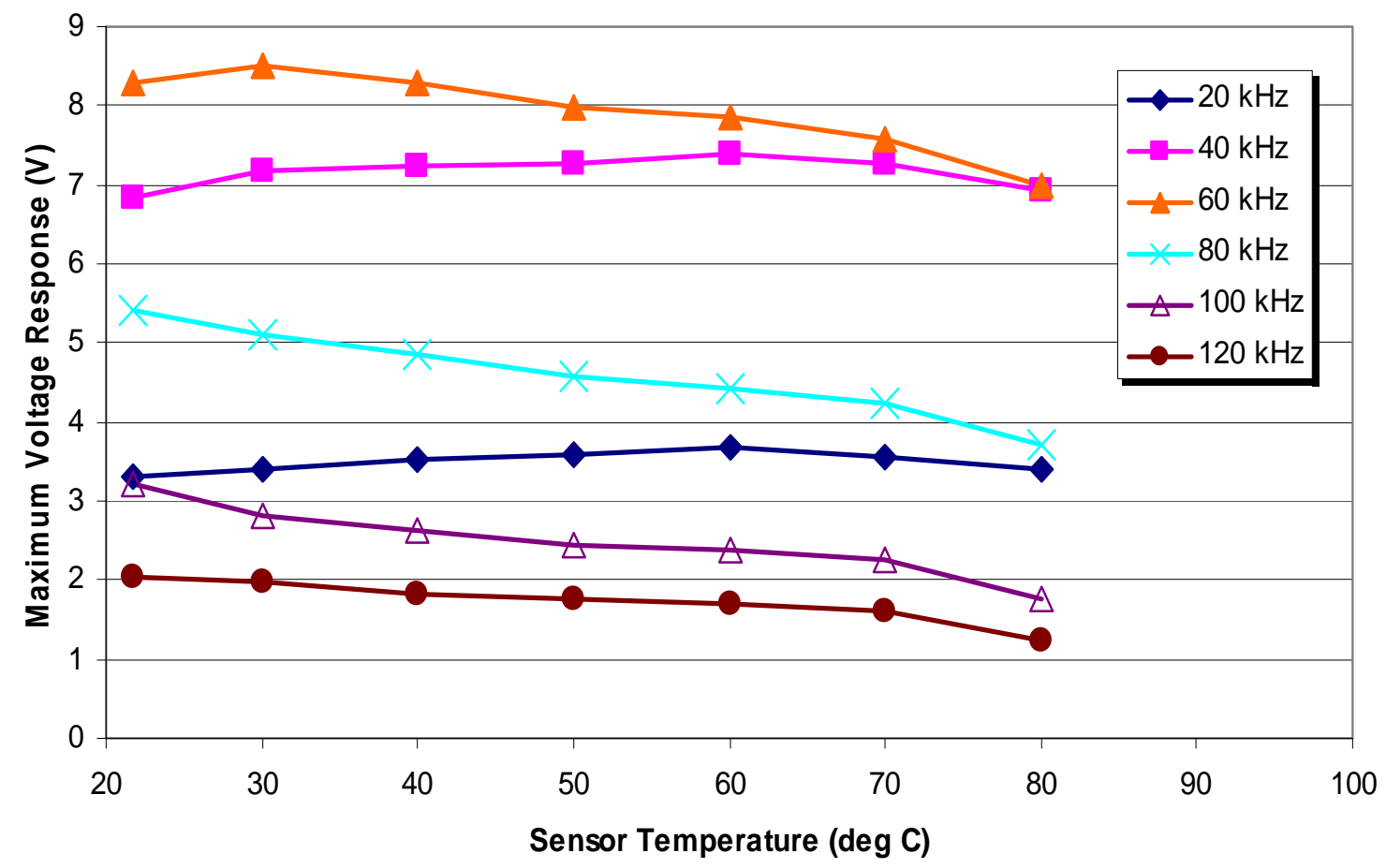

Figure 3-7-1. S1 maximum time response vs. temperature, E2 excitation. 


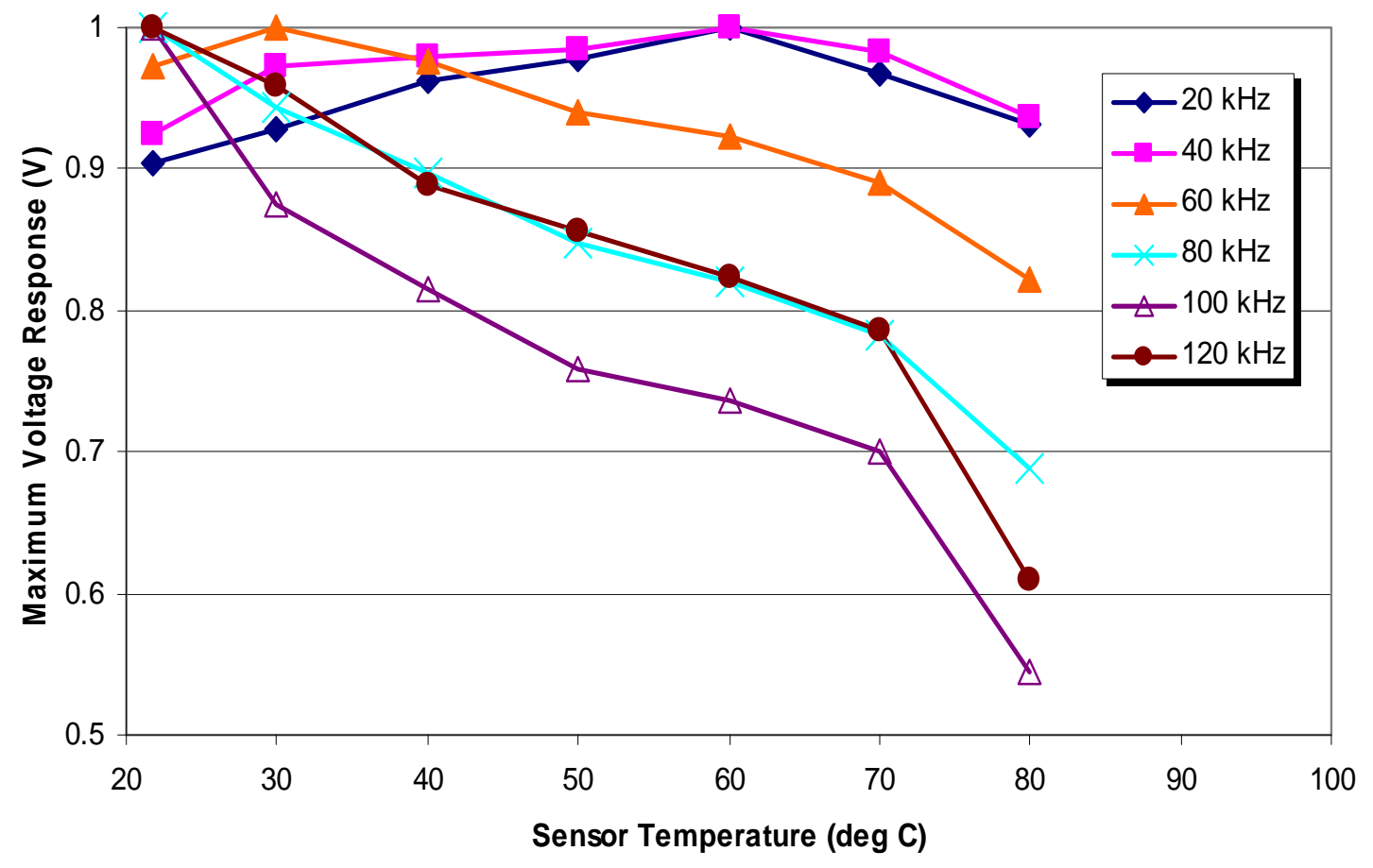

Figure 3-7-2. S1 normalized time response vs. temperature, E2 excitation. 


\section{Chapter 4: Damage Discrimination}

An expert, rule-based discriminator was built in an attempt to exploit pattern recognition of the experimental results to fulfill two main goals in a simple manner:

1) Localize damage in the composite structure's four quadrants.

2) Quantify the damage due to bolt torque loss in a single bolt.

As outlined in the previous chapter, pattern recognition of $D I$ trends provided insight to the localization of damage within a quadrant. In particular, patterns shown in the sideby-side comparison of average normalized damage index over four excitation configurations were the most useful in localizing a single damaged bolt. These plots were previously shown for Bolts 0, 1 and 2 in Figure 3-1-33, Figure 3-2-17 and Figure 3$3-17$, respectively.

\subsection{Damage index pattern recognition}

Upon the completion of all four excitation cases (E1, E2, E3, E4) of a single damaged bolt, the $D I$ was organized into a grid; the $D I$ grid for Bolt $0,100 \%$ torque loss is shown in Table 4-1-1.

Table 4-1-1. DI grid, 100\% B0 torque loss.

\begin{tabular}{|c|c|c|c|c|}
\cline { 2 - 5 } \multicolumn{1}{c|}{} & S1 & S2 & S3 & S4 \\
\hline E1 excitation & -- & 0.04 & 0.53 & 0.41 \\
\hline E2 excitation & 0.09 & -- & 0.68 & 0.40 \\
\hline E3 excitation & 0.59 & 0.45 & -- & 0.06 \\
\hline E4 excitation & 0.55 & 0.25 & 0.09 & -- \\
\hline SUM & 1.14 & 0.70 & 1.21 & 0.81 \\
\hline
\end{tabular}


The shaded cells in Table 4-1-1 indicate $D I$ values from situations where the sensor shares the same composite panel as the origin of excitation signal. For example, excitation at E1 was sensed by S2; this excitation wave does not cross the bolted joint before being sensed at S2. In the previous chapter, it was determined that same-panel excitation/sensing response does not provide insight into the damage level within the joint. It was only in situations where the excitation wave traveled through the joint that a sensing response would provide details into the bolt damage levels. The MATLAB discriminator does not consider these $D I$ values in subsequent calculations and reasoning.

The summation of non-shaded columns in Table 4-1-1 provided insight into the likely damaged area. Comparing the $D I$ sums at each sensor:

$$
D I_{S 3}>D I_{S 1}>D I_{S 4}>D I_{S 2}
$$

As previously stated, a rule of thumb indicates higher likelihood of damage in the vicinity of the sensor with highest DI. From the summations in Table 4-1-1, it was determined that the highest likelihood of damage was geometrically closest to S3, in Quadrant 3. This confirmed the actual location of damage. Damage index grids were compiled for Bolt 0 , Bolt 1, Bolt 2 over all torque loss values $(100 \%, 75 \%, 50 \%$, and $25 \%$ ) to determine whether Quadrant 3 had the highest likelihood of damage. The DI summations were ranked for each grid; nearly all grids confirmed the correct damage quadrant. A single inaccurate ranking occurred for Bolt $1,75 \%$ torque loss; the grid is shown in Table 4-1-2. 
Table 4-1-2. DI grid, 75\% B1 torque loss.

\begin{tabular}{|c|c|c|c|c|}
\cline { 2 - 5 } \multicolumn{1}{c|}{} & S1 & S2 & S3 & S4 \\
\hline E1 excitation & -- & 0.05 & 0.18 & 0.18 \\
\hline E2 excitation & 0.05 & -- & 0.20 & 0.11 \\
\hline E3 excitation & 0.18 & 0.19 & -- & 0.09 \\
\hline E4 excitation & 0.30 & 0.20 & 0.08 & -- \\
\hline SUM & 0.48 & 0.39 & 0.38 & 0.29 \\
\hline
\end{tabular}

Comparing the $D I$ sums at each sensor:

$$
D I_{S 1}>D I_{S 2}>D I_{S 3}>D I_{S 4}
$$

From the summations in Table 4-1-2, it was determined that the highest likelihood of damage was geometrically closest to $\mathrm{S} 1$, in Quadrant 1 . This did not correlate with the actual location of damage in Quadrant 3.

Comparing the $D I$ values in Table 4-1-1 and 4-1-2, there was a noticeable difference in $D I$ magnitude. Table 4-1-2 shows the $D I$ for a $75 \%$ torque loss case, while Table 4-1-1 shows the $D I$ for a total loss in bolt torque. Table 4-1-3 shows the $D I$ grid for Bolt 2, 25\% torque loss.

Table 4-1-3. DI grid, 25\% B2 torque loss.

\begin{tabular}{|c|c|c|c|c|}
\cline { 2 - 5 } \multicolumn{1}{c|}{} & S1 & S2 & S3 & S4 \\
\hline E1 excitation & -- & 0.05 & 0.11 & 0.12 \\
\hline E2 excitation & 0.01 & -- & 0.12 & 0.08 \\
\hline E3 excitation & 0.11 & 0.14 & -- & 0.07 \\
\hline E4 excitation & 0.06 & 0.08 & 0.02 & -- \\
\hline SUM & 0.17 & 0.22 & 0.23 & 0.20 \\
\hline
\end{tabular}


The magnitude of the four $D I$ summations, $D I_{\text {mag }}$ were calculated according to:

$$
\left(D I_{m a g}\right)^{2}=\left(D I_{S U M, S I}\right)^{2}+\left(D I_{S U M, S 2}\right)^{2}+\left(D I_{S U M, S 3}\right)^{2}+\left(D I_{S U M, S 4}\right)^{2}
$$

Table 4-1-4 shows the magnitude calculations for the DI grids in Tables 4-1-1, 4-1-2, and 4-1-3. It was determined that the magnitude of $D I$ is directly proportional to damage severity due to bolt torque loss.

Table 4-1-4. DI $I_{\text {mag }}$; B0 100\% torque loss, B1 75\% torque loss, B2 25\% torque loss.

\begin{tabular}{|c|c|c|c|}
\hline & $\mathrm{B} 0,100 \%$ & $\mathrm{~B} 1,75 \%$ & $\mathrm{~B} 2,25 \%$ \\
\hline DI magnitude & 1.98 & 0.61 & 0.17 \\
\hline
\end{tabular}

\subsection{Level 2: Damage localization algorithm}

The MATLAB discriminator code is an expert-guided, rule-based series of ifstatements that examine the damage index values and rank a specified set of values. The main goal was to localize a damage site according to recognized $D I$ trends. To distinguish between Quadrants 1/3 (top half of the composite panel, in-between sensors S1 and S3) and Quadrants 2/4 (bottom half of the composite panel, in-between sensors S2 and S4) the discriminator focuses on straight-across signals, as illustrated in Figure 4-1-1. This technique was based on the recognized patterns and trends collected during analysis of a single bolt torque loss scenario. This process, as well as the $D I$ grid for a single damage scenario is outlined in section 4.1. This grid was input into the MATLAB discrimination code to exploit the $D I$ trends; a sample input is shown in the Appendix. 


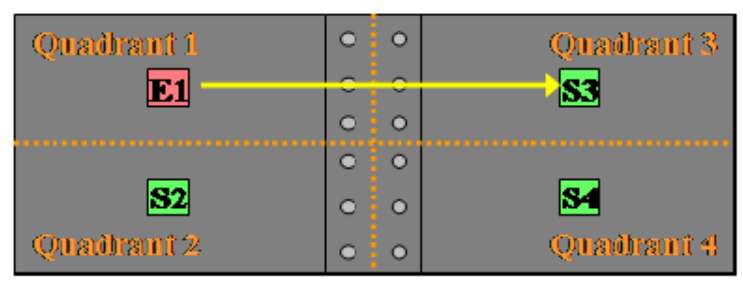

(2) $\mathrm{E1}$ signel, sensod by $\mathrm{s3}$

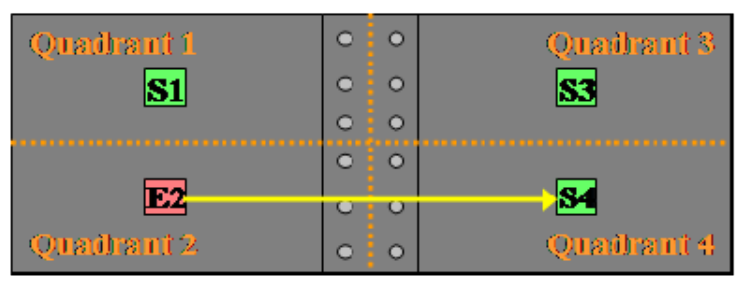

(c)F2 signel, sensed by S4

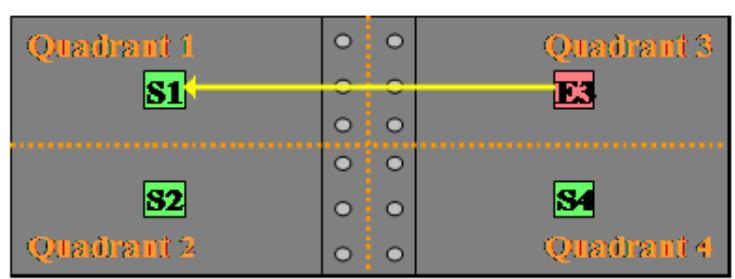

(b) ES signil sensed by 81

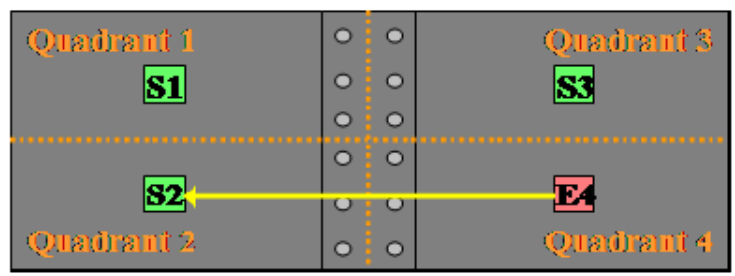

(d) B4 signe-1, sensed by 52

Figure 4-1-1. Path of straight-across excitation.

Figures 4-1-1(a) and 4-1-1(b) show the excitation/sensing path used to calculate $D I_{E 1, S 3}$ and $D I_{E 3, S 1}$. Table 4-1-1 is shown again with highlighted $D I$ information from the excitation/sensing scenarios in Figure 4-1-1(a) and Figure 4-1-1(b). The MATLAB discriminator used these values to localize damage within Quadrants 1 or 3.

Table 4-1-1. DI grid, 100\% B0 torque loss.

\begin{tabular}{|c|c|c|c|c|}
\hline & S1 & 52 & $\mathbf{s 3}$ & 54 \\
\hline E1 excitation & & 0 & 0.53 & 0.41 \\
\hline E2 excitation & 6 & & 0.68 & 0.40 \\
\hline E3 excitation & 0.59 & 0.45 & & $\ldots$ \\
\hline E4 excitation & 0.55 & 0.25 & 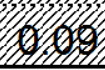 & \\
\hline SUM & 1.14 & 0.70 & 1.21 & 0.81 \\
\hline
\end{tabular}


Similarly, sensing information and damage index values obtained from the experimental configurations in Figures 4-1-1(c) and 4-1-1(d) were used by the discriminator to localize damage within Quadrants 2 and 4. Table 4-1-1 is shown again with highlighted DI information from the excitation/sensing scenarios in Figure 4-1-1(c) and Figure 4-1-1(d).

Table 4-1-1. DI grid, 100\% B0 torque loss.

\begin{tabular}{|c|c|c|c|c|}
\hline & s1 & $\mathbf{S 2}$ & S3 & S4 \\
\hline E1 excitation & HI & 014 & 0.53 & 0.41 \\
\hline E2 excitation & 6 & & 0.68 & 0.40 \\
\hline E3 excitation & 0.59 & 0.45 & f & 0 \\
\hline E4 excitation & 0.55 & 0.25 & 60 & Iffe \\
\hline SUM & 1.14 & 0.70 & 1.21 & 0.81 \\
\hline
\end{tabular}

As a simple rule, a higher damage index represents a higher likelihood of damage present within the vicinity of active sensing. If the damage indices obtained from active sensing agents in Quadrants 1/3 are higher than those obtained from the signals in Quadrants 2/4, the damage is more likely to be present in Quadrants 1 or 3. Using Table 4-1-1 as an example, the $D I$ values highlighted in red indicated a higher likelihood of damage in Quadrants 1 and 3. The MATLAB program examines $D I$ and outputs a simple statement indicating which of the quadrant pairs is most likely to contain the damaged bolt due to torque loss.

Once the discriminator determines which sensor pair the damage lies between, the next goal was the discrimination between the left and right quadrant within the top or bottom half of the composite jointed panel. To discriminate between the left and right 
quadrant, the diagonally-across signals provided insight. Figure 4-1-2 shows the excitation/sensing path of the diagonal excitation.

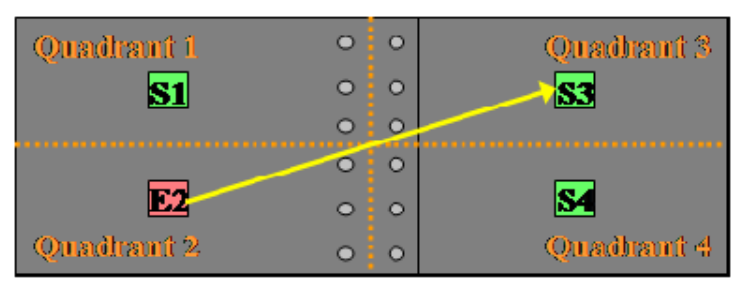

(G) $\mathrm{H2}$ sign-1, senued by 83

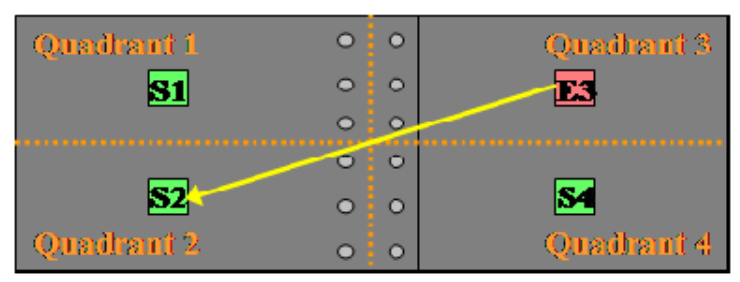

(c) $\mathrm{E3}$ signil, sensed by 52

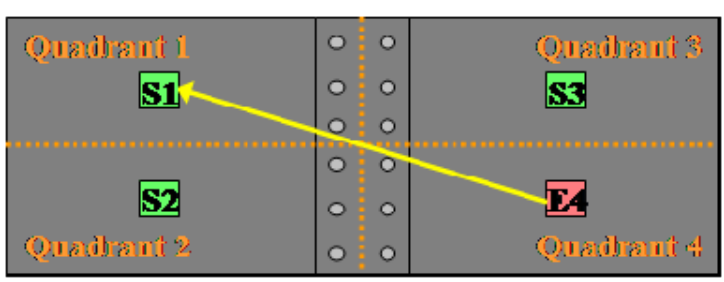

(b) 84 signel, sensed by 81

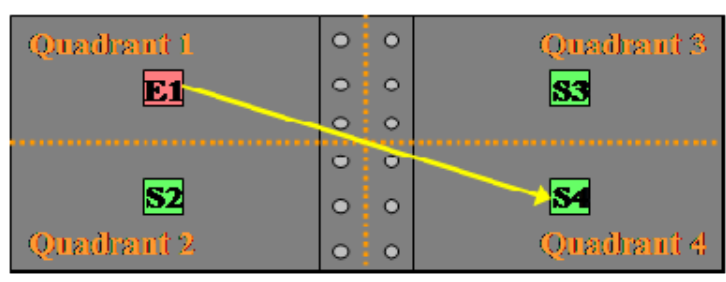

(d) El signil, sensed by S4

Figure 4-1-2. Path of diagonally-across excitation on the composite jointed panel.

An actuator's proximity to damage negates the insight provided from scenarios in Figures 4-1-2(c) and 4-1-2(d); it was shown in previous results that actuators with close proximity to damage yielded damage index trends that were less clear as compared to results from actuators that were further from a known area of damage. For example, the information in Table 4-1-1 indicated damage in-between sensing agents S1 and S3; the most useful sensing information for this possible damage scenario comes from Figures 4-1-2(a) and 4-1-2(b). Figure 4-1-2(a) shows the wave path used to calculate $D I_{E 2, S 3}$; Figure 4-1-2(b) shows the wave path for $D I_{E 4, S I}$. Table 4-1-1 is shown again with these values highlighted. The general rule still applies; a higher damage index implies a greater probability of damage within the sensing vicinity. 
Table 4-1-1. DI grid, 100\% B0 torque loss.

\begin{tabular}{|c|c|c|c|c|}
\hline & s1 & s2 & $\mathbf{S 3}$ & 54 \\
\hline E1 excitation & & 1) & 0.53 & 0.41 \\
\hline E2 excitation & (6) & Ifflla, & 0.68 & 0.40 \\
\hline E3 excitation & 0.59 & 0.45 & VIIIIII & 6 \\
\hline E4 excitation & 0.55 & 0.25 & 0 & SIIIs \\
\hline SUM & 1.14 & 0.70 & 1.21 & 0.81 \\
\hline
\end{tabular}

A higher damage index obtained from the scenario in Figure 4-1-2(a) as compared to the damage index from scenario in Figure 4-1-2(b) would imply the highest likelihood of damage in Quadrant 3. Alternately, a higher damage index obtained from scenario in Figure 4-1-2(b) as compared to scenario in Figure 4-1-2(a) will predict with greater confidence that the damaged bolt lies in Quadrant 1. For this example, $D I_{E 2, S 3}$ was higher than $D I_{E 4, S I}$; thus, the greater likelihood of damage was in Quadrant 3. This agreed with the actual damage location in Bolt 0 for $100 \%$ torque loss.

A graphical method was used to more plainly display the results over all experimental damage scenarios. The $D I$ grids for bolts in Quadrant 3 (Bolts 0, 1 and 2) and Quadrant 1 (Bolt 9, 10, and 11) were used to compile a plot over all torque loss values. As the experimentation and analysis of Bolt 6 indicated, the DI methodology produced symmetrical results; this reasoning allowed the approximation of $D I$ for bolts in Quadrant 1 based on Quadrant 3 damage information, and thus no further timeconsuming experimentation was needed. The results obtained from multiple damage scenarios in Bolt 0 (top bolt in Quadrant 3), Bolt 1 (middle bolt in Quadrant 3), Bolt 2 (bottom bolt in Quadrant 3), Bolt 9 (bottom bolt in Quadrant 1), Bolt 10 (middle bolt in 
Quadrant 1), and Bolt 11 (top bolt in Quadrant 1) were plotted, using the DI from in $D I_{E 2, S 3}$ on the x-axis and the $D I_{E 4, S 1}$ on the y-axis. Figure 4-1-3 shows $D I_{E 2, S 3}$ vs. $D I_{E 4, S 1}$ for all six bolts within Quadrants 1 and 3 for all experimental damage scenarios: 100\% torque loss (circle), $75 \%$ torque loss (square), 50\% torque loss (triangle) and $25 \%$ torque loss (diamond). The open markers represent damage indices from Bolts 9, 10, 11 (Quadrant 1). The closed markers represent damage indices taken from Bolts 0, 1 and 2 (Quadrant 3). The black 45-degree ray graphically represents the separation between areas where Quadrant 3 sensing information is smaller or greater than Quadrant 1 information; a DI point below the threshold indicates a higher likelihood of damage in Quadrant 3, while a DI point above the threshold indicates a higher likelihood of damage in Quadrant 1.

The groupings of DI markers representing 100\% torque loss have a clear separation; the average coordinate location of the two separated groups is plotted as an "X" and "+" and noted for each grouping. As the torque loss values decrease, the separation between Quadrants 1 and $3 D I$ groupings is less clear. Independent of torque loss, each group average lays on the side of the 45-degree ray which correctly corresponds to the damaged quadrant. The successful geometric localization of damage within the composite assembly's four quadrants fulfilled the requirements for a Level 2 prediction method. 


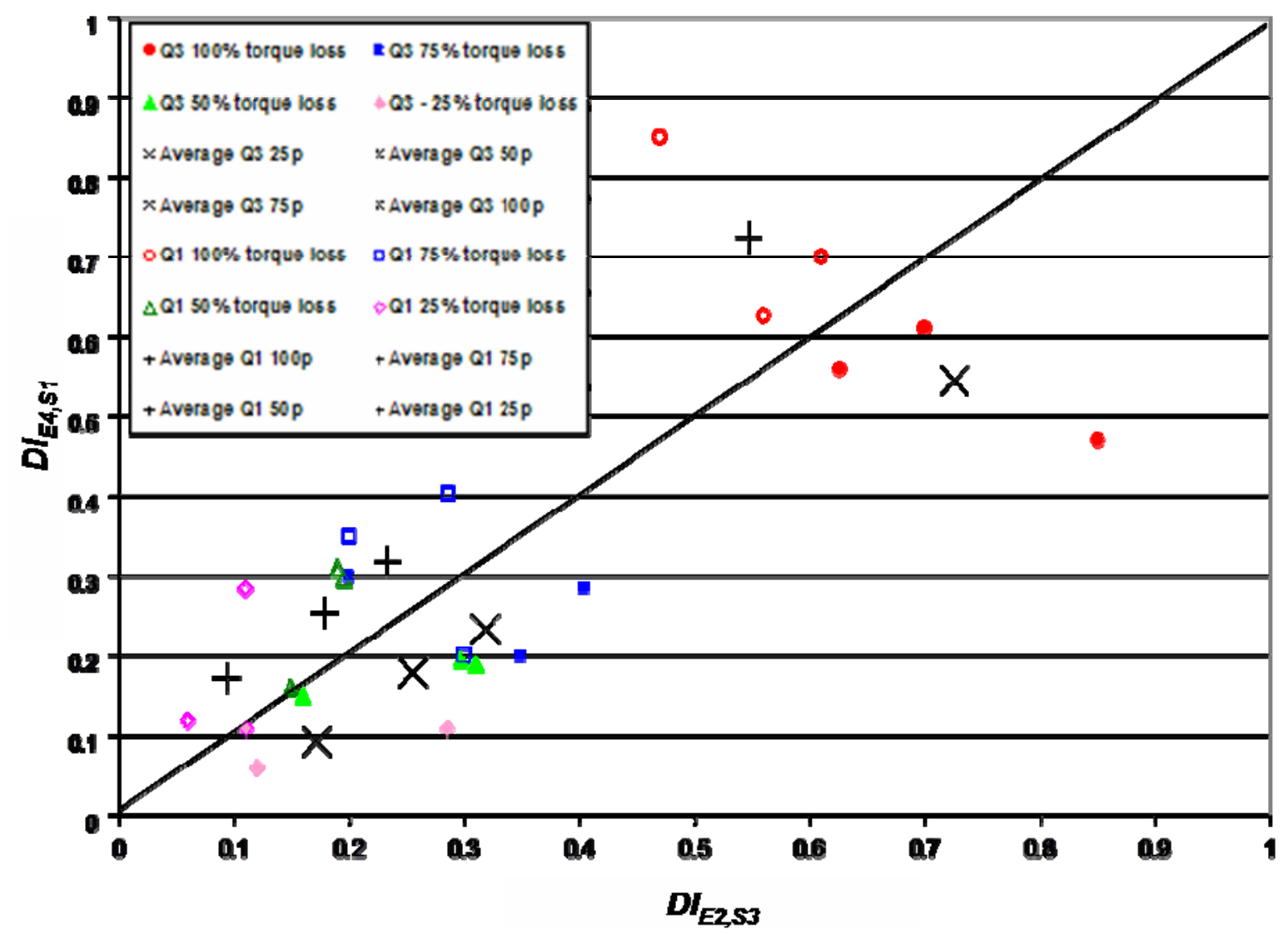

Figure 4-1-3. Quadrant 1 vs. Quadrant 3 damage index sensing information.

\subsection{Level 3: Damage quantification algorithm}

To fulfill a Level 3 prediction method for structural health monitoring of the composite lap-joint assembly, a statistical threshold was implemented to discriminate between torque loss values. Threshold bands were calculated using the groupings' average markers. A Euclidean distance classifier [27] was employed to determine the central radius between the $100 \%$ torque loss and $75 \%$ torque loss average markers. The general distance formula between two points follows:

$$
r_{2,1}=r_{1}+1 / 2\left(r_{2}-r_{1}\right)
$$


This calculation was performed to find the center radius between $100 \%$ and $75 \%$ torque loss groupings, $75 \%$ and $50 \%$ torque loss groupings, and $50 \%$ and $25 \%$ torque loss groupings. Figure 4-1-4 shows the same plot of $D I$ in Figure 4-1-3 with the threshold bands that separate damage levels due to torque loss. A fourth band, shown in grey, separates the graphical area of indistinguishable damage. As outlined in Chapter 4, experimental results indicated quantifiable sensing capability above a $D I_{\text {total }}$ value of 0.1 . It was determined that damage index values below 0.1 would not give clear insight into the damage presence or location within the composite joint.

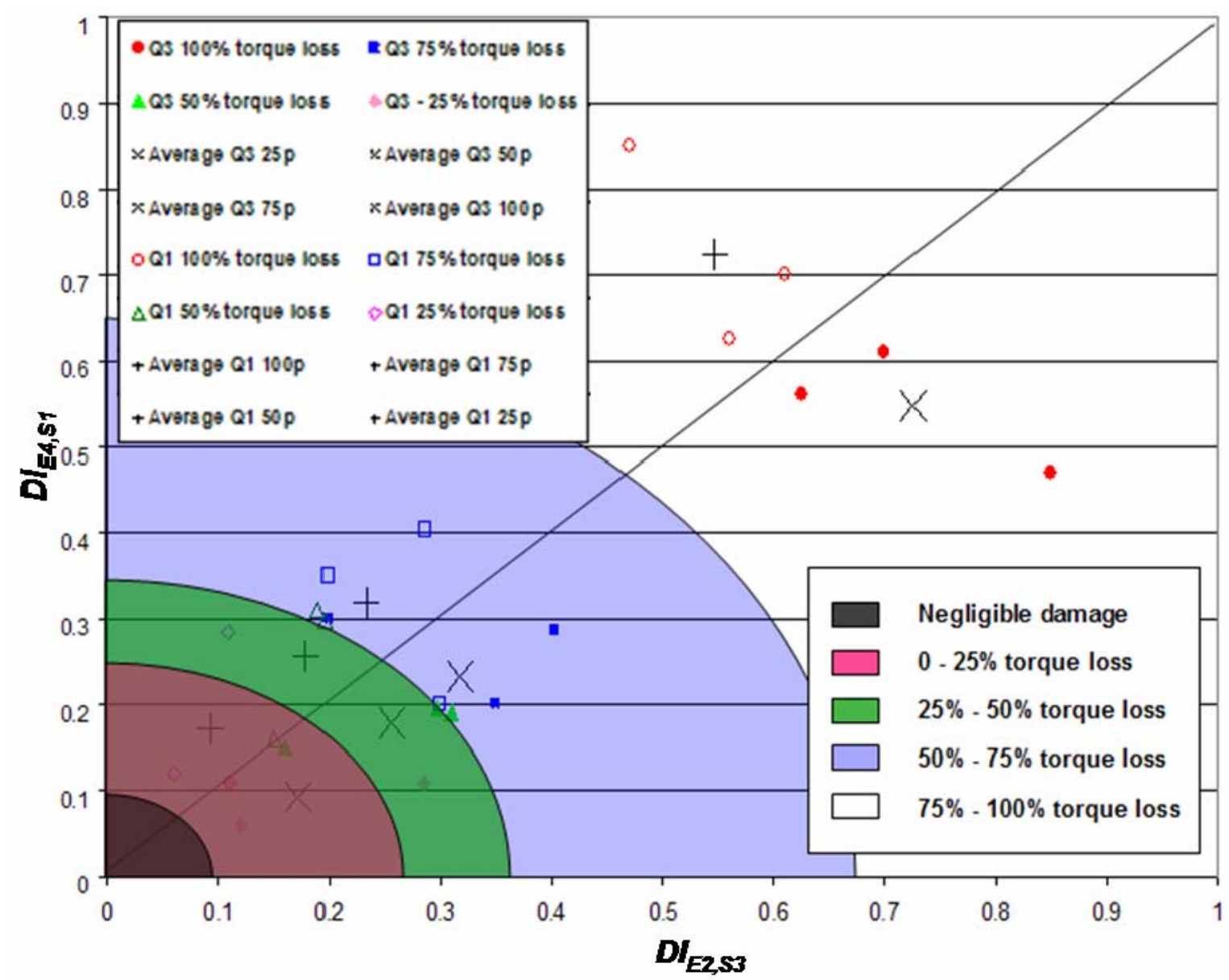

Figure 4-1-4. Quadrant 1 vs. Quadrant 3 damage with damage threshold bands. 
To investigate the confidence of these threshold predictions, a series of validation experiments were performed. This supplemental experimentation was conducted in the same manner as the experimentation described in Chapters 2 and 3. Multiple levels of damage were implemented on all six bolts within Quadrants 1 and 3. The validation run matrix is listed in Table 4-1-5.

Table 4-1-5: Validation experimental run matrix.

\begin{tabular}{|c|c|c|c|}
\hline Run & Damaged Bolt & Damaged Quadrant & Percent torque loss \\
\hline 1 & 9 & 1 & 100 \\
\hline 2 & 10 & 1 & 100 \\
\hline 3 & 11 & 1 & 100 \\
\hline 4 & 0 & 3 & 100 \\
\hline 5 & 1 & 3 & 100 \\
\hline 6 & 2 & 3 & 100 \\
\hline 7 & 10 & 1 & 75 \\
\hline 8 & 2 & 3 & 75 \\
\hline 9 & 9 & 1 & 50 \\
\hline 10 & 0 & 3 & 50 \\
\hline 11 & 11 & 1 & 25 \\
\hline 12 & 1 & 3 & 25 \\
\hline
\end{tabular}

For each validation run, $D I_{E 2, S 3}$ and $D I_{E 4, S 2}$ was plotted on Figure 4-1-4 to observe their location within the prediction bands. The validation run results are shown in Figure 4-1-5. All result markers were located on the correct side of the 45-degree threshold line which verified the actual quadrant of damage. Additionally, all but one of the validation run results was located in the shaded band which correctly corresponded to the torque loss level. One outlier existed with Run 8 which was the result of $25 \%$ torque loss in Bolt 2; this result was found in the $25 \%-50 \%$ torque loss prediction band. It has been previously stated in Chapter 3 that Bolt 2 yielded results that were less clear relative to Bolts 0 and 1, which are also in Quadrant 3. A potential reason for this was because of 
its proximity to Quadrant 4 . Also, since $25 \%$ torque loss is relatively low, it is reasonable to state that the discriminator can predict with higher confidence the location and quantification of damage due torque loss values above $50 \%$. The successful development of a comprehensive localization and damage quantification technique fulfilled the requirements for a Level 3 prediction methodology.

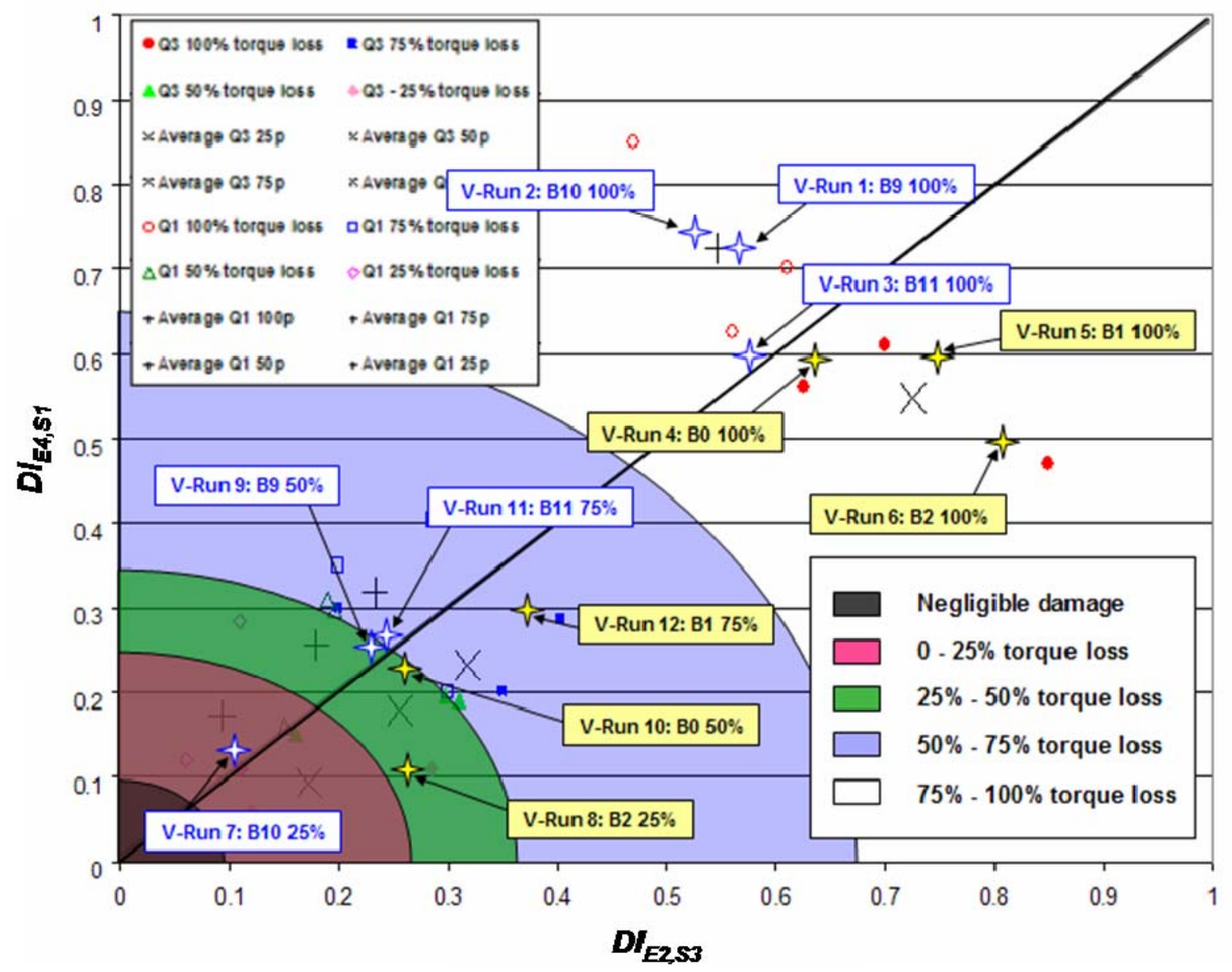

Figure 4-1-5. Quadrant 1 vs. Quadrant 3 discriminator validation run results. 


\section{Chapter 5: Concluding Remarks}

The objective of this research was to utilize the Lamb wave method to formulate a cohesive structural health monitoring methodology for the composite lap-joint assembly. Sensing information was collected to construct a sizeable database of baseline and damage information with respect to single and dual-bolt torque loss over a range of damage levels and multiple locations within the composite joint. Exploitation of this database information along with key features inherent to the structural system allowed for the development of a prediction Level 3 damage diagnostic. To realize this aim, several experimental and analytical elements were employed:

1) The damage index for an individual sensor (Figure 3-1-5 and Figure 3-1-6) displayed a higher sensitivity to bolt torque loss as compared to the sensitivity due to excitation frequency.

2) Damage index information from wave paths which crossed over the joint (Figure 4-1-1 and Figure 4-1-2) provided excellent insight into the damage levels within the joint. Damage indices from waves that did not cross the joint (Figure 3-4-15) provided little insight into the damage present within the bolted joint. This pattern recognition was exploited to attain damage prediction Level 1.

3) The damage index taken from excitation piezos that were far from an implemented damage site provided useful insight toward the localization of damage. Pattern recognition of the damage index as a key feature led to the successful localization of a damaged bolt within one of the 
composite structure's four quadrants. This methodology attained damage prediction Level 2 for the composite jointed system.

4) Plotting total damage index (Figure 4-1-3) yielded a clear separation between results of higher damage levels. As damage due to torque loss decreased (approximately $25 \%$ torque loss), damage index provided the location of damage with less clarity.

5) Total damage index was used to build a rule-based discriminator which accurately predicted the damaged quadrant and quantity of damage due to bolt torque loss in 11 validation cases (Figure 4-1-5). A $12^{\text {th }}$ validation case resulted in an outlier. This discrimination method provided a damage prediction Level 3 for the composite jointed system.

The results of this research indicate a strong potential for using the Lamb wave method along with simple discrimination techniques for damage due to torque loss in the composite lap-joint assembly. Further, the $D I$ proved to be a robust feature for the development of a damage diagnostic up to a Level 3 prediction. This research did not examine types of damage beyond single and dual-bolt torque loss. Extending the Lamb wave method to applications where the composite jointed panel undergoes other damaged scenarios, such as damage due to machined cracks, composite delamination or added mass is suggested as a topic for future investigation. Further, it is suggested that this diagnostic methodology be applied to structures composed of other materials, both composite and isotropic.

Supplemental experimentation was performed to examine the smart sensor array's sensing capability over time. Over the five month duration of this research, the baseline 
sensing information showed a slight variation. The damage index relies entirely the difference between damaged and undamaged sensing information. The idea that even a slight variance in baseline information would affect the overall damage index should not be overlooked. The results in section 3.6 give rise to the opinion that additional investigation should be devoted to understanding this phenomenon and its impact into the use of damage index as a key discrimination feature. The capabilities of the discriminator would be compromised if the damage index were to experience an unpredictable shift due to smart sensor aging. To determine the sensor array's operational capability, a life-span of acceptable use should be quantified.

Additional experimentation examined the sensing capability of the piezo-actuators at elevated temperatures. The results in section 3.7 indicate a shift in time signal response as the sensor temperature was increased. A decrease in voltage response was potentially due to decreased strain as the piezo became malleable at higher temperatures. In this research study, the smart sensor array and composite jointed system did not experience a large temperature variation within the laboratory environment. However, if a similar experimental set-up were used in a laboratory or operational environment which did experience a large temperature variation, the effects of temperature should be investigated further. Since the damage index as a key feature relies entirely on the sensing response, any change may affect the overall damage index, and thus the discrimination capabilities of the diagnostic system. 


\section{Appendix}

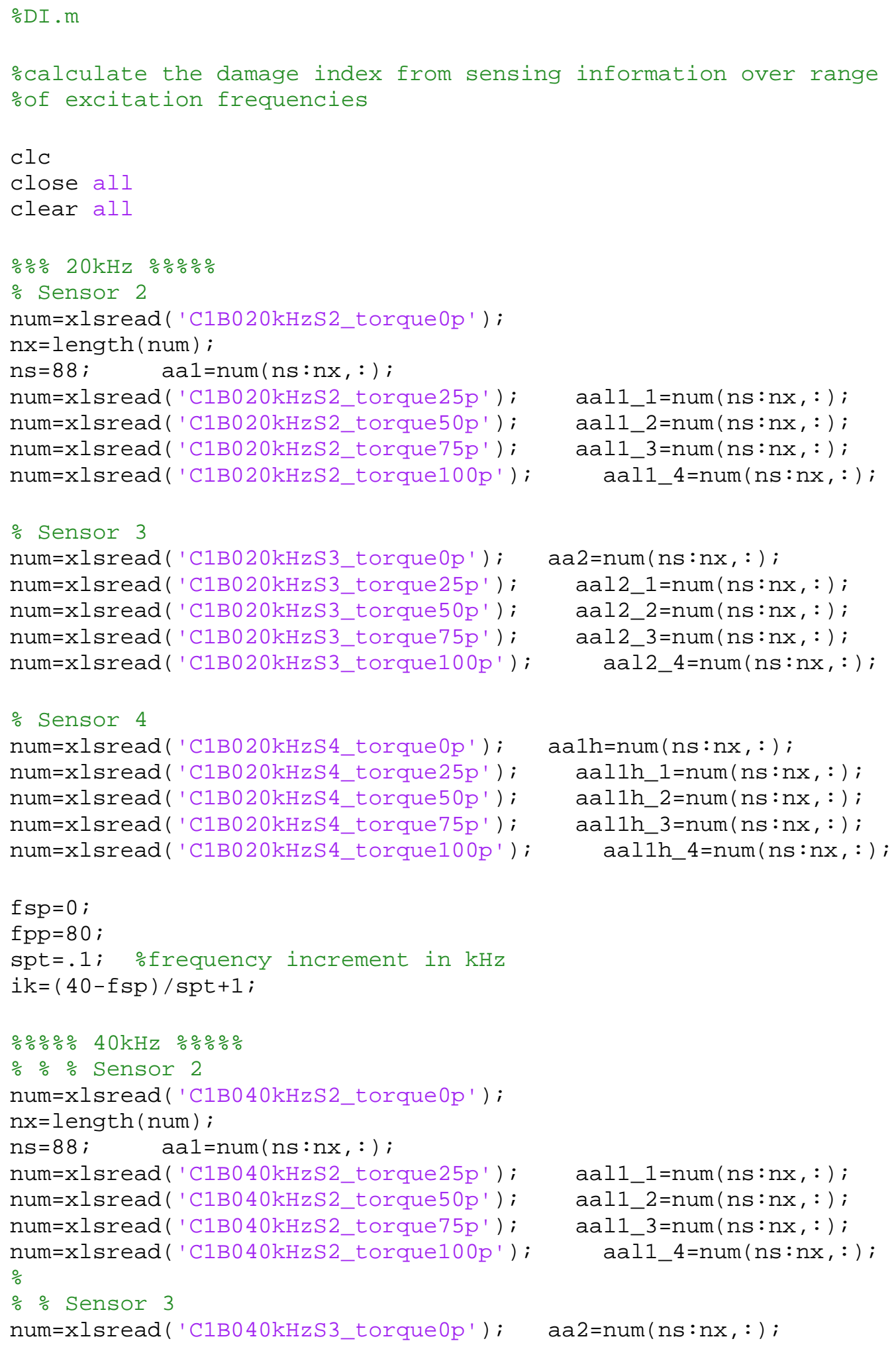




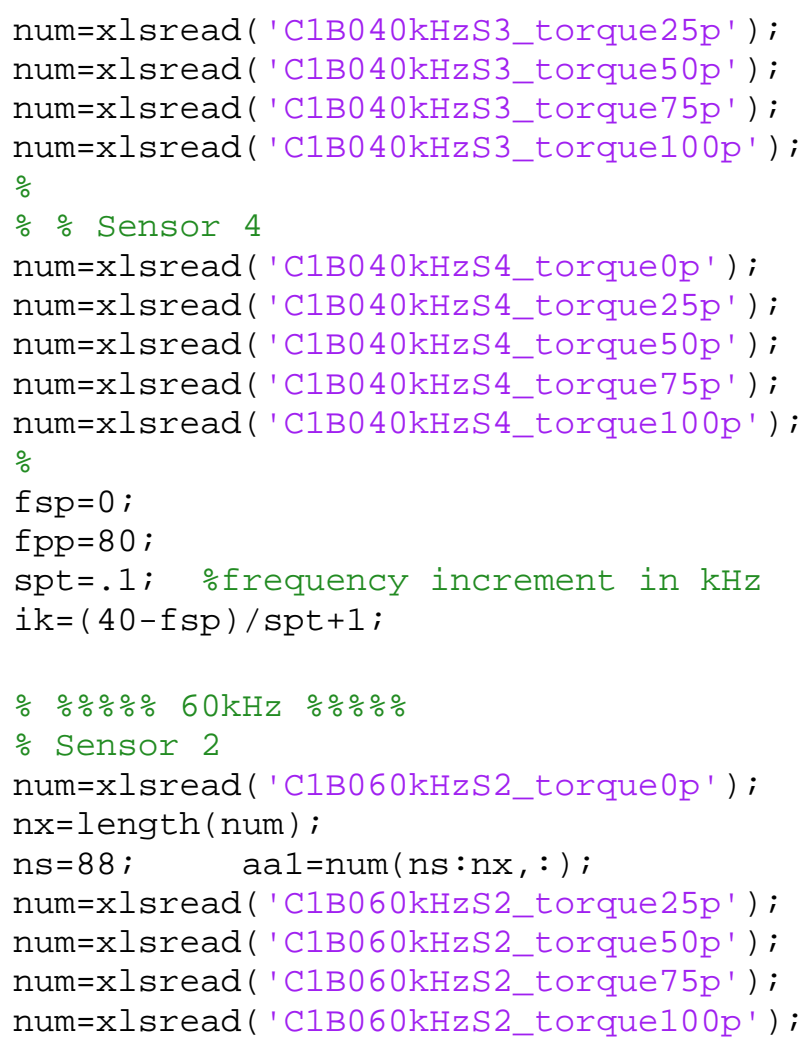

aal2_1=num(ns: nx, : ); aal2_2=num (ns: nx, : ); aal2_3=num (ns: nx, : ); aal2_4=num(ns:nx, : );

aa1h=num(ns:nx, : ); aal1h_1=num(ns:nx, : ); aal1h_2=num(ns:nx, : ); aal1h_3=num(ns:nx, : ); aal1h_4=num(ns:nx,: );

aal1_1=num(ns: nx, : ); aal1_2=num(ns:nx, : ); aal1_3=num $(\mathrm{ns}: \mathrm{nx},:)$; aal1_4=num(ns:nx, : );

aa2=num(ns: nx, : ) ; aal2_1=num(ns:nx, : ); aal2_2=num (ns: nx, : ); aal2_3=num (ns: nx, : ); aal2_4=num(ns:nx, : );

aa1h=num(ns:nx, : ); aal1h_1=num(ns:nx, : ) ; aal1h_2=num(ns: nx, : ); aal1h_3=num (ns: nx, : ); aal1h_4=num(ns:nx, : );

aal1_1=num(ns: nx, : ) ; aal1_2=num (ns: nx, : ); aal1_3=num (ns: nx, : ); aal1_4=num(ns:nx, : ) ; 


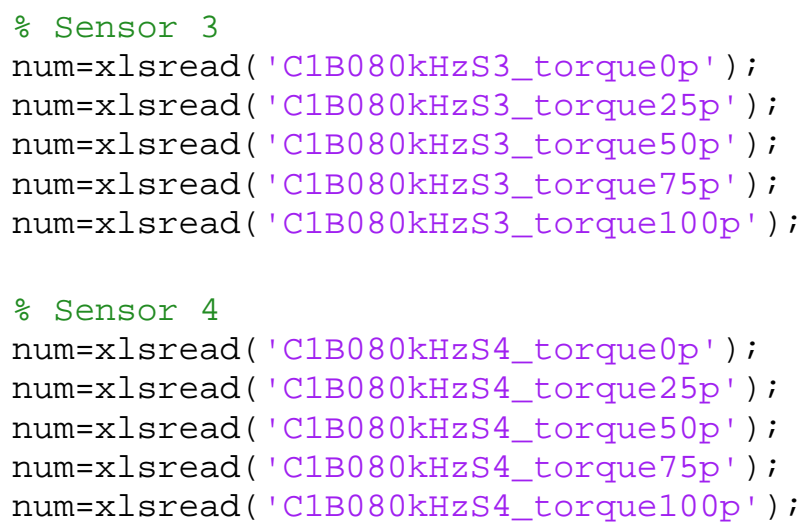

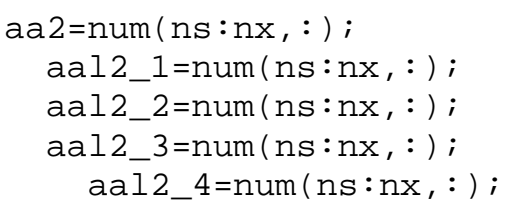

aal1_1=num(ns: nx, : ); aal1_2=num(ns:nx, : ); aal1_3=num (ns:nx, : );

aal1_4=num(ns:nx, : ); aa1h=num (ns:nx, : ); aal1h_1=num(ns:nx, : ); aal1h_2=num (ns:nx, : ); aal1h_3=num (ns:nx, : ); aal1h_4=num(ns:nx, : );

aal1_1=num(ns:nx, : ); aal1_2=num $(\mathrm{ns}: \mathrm{nx},:)$; 


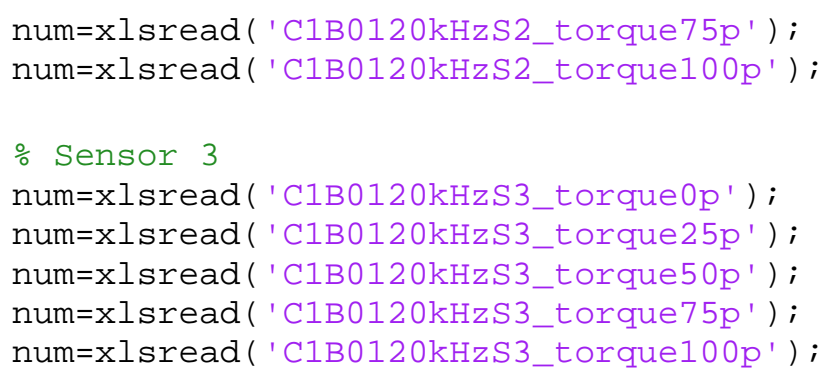


[ssl, ffl, ttl, ppl2_4]=spectrogram(aal2_4(1:kp,2),nfft, ovp, [fsp:spt:fpp] * $1 \mathrm{e} 3, \mathrm{Fs})$;

[ssl, ffl, ttl, pp1h]=spectrogram(aa1h(1:kp,2), nfft, ovp, [fsp:spt:fpp] *1e3, Fs );

[ssl,ffl,ttl,ppl1h_1]=spectrogram(aal1h_1(1:kp,2),nfft,ovp, [fsp:spt:fpp ] $1 \mathrm{e} 3, \mathrm{Fs})$;

[ssl,ffl, ttl,ppl1h_2]=spectrogram(aal1h_2(1:kp,2),nfft,ovp, [fsp:spt:fpp ]*1e3,Fs);

[ssl,ffl, ttl,ppl1h_3]=spectrogram(aal1h_3(1:kp,2),nfft,ovp, [fsp:spt:fpp ] $1 \mathrm{e} 3, \mathrm{Fs})$;

[ssl,ffl, ttl,ppl1h_4]=spectrogram(aal1h_4(1:kp,2),nfft,ovp, [fsp:spt:fpp ]*1e3,Fs);

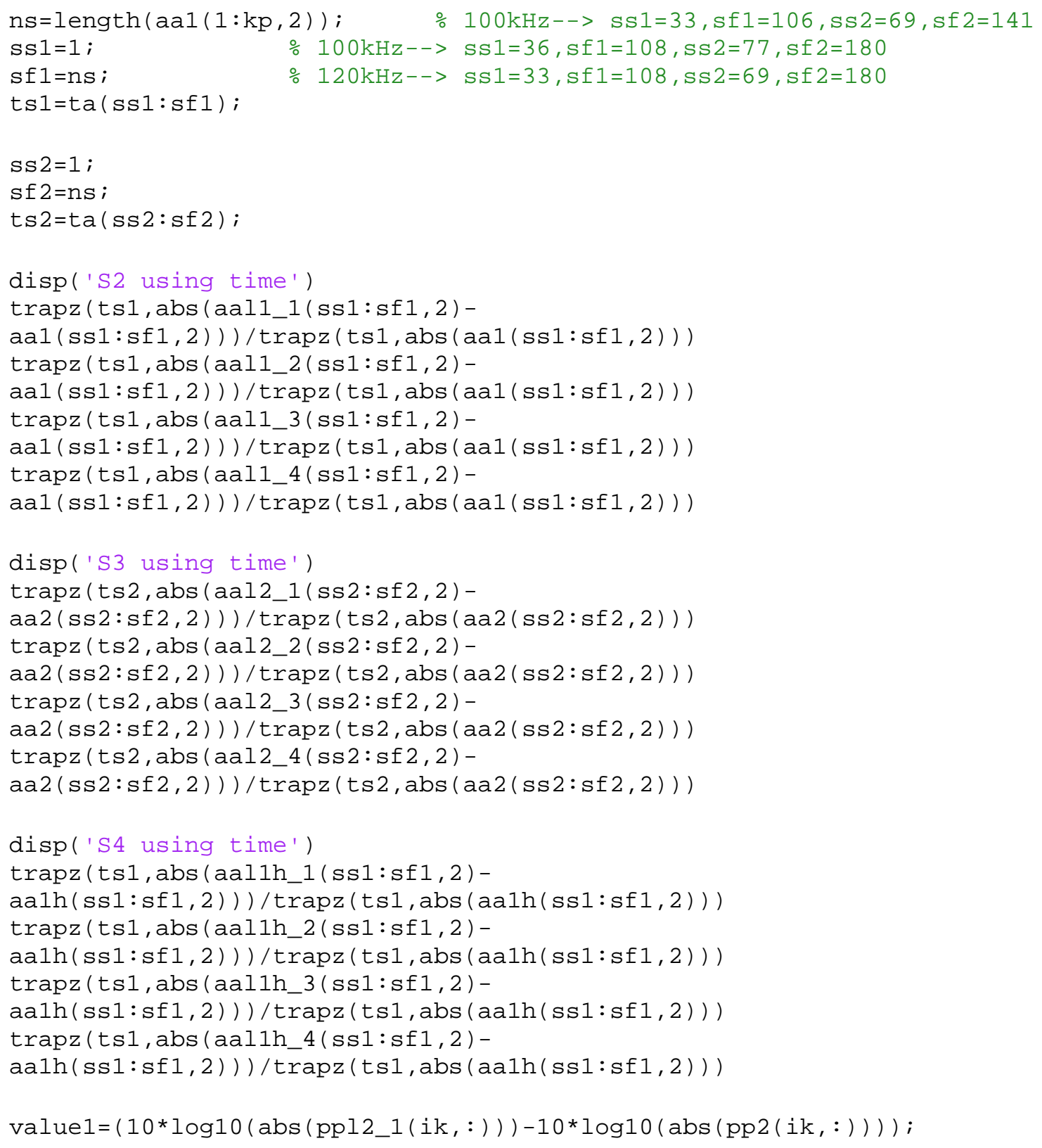


value2 $=\left(10 * \log 10\left(\operatorname{abs}\left(p p 12 \_2(i k,:)\right)\right)-10 * \log 10(\operatorname{abs}(p p 2(i k,:)))\right)$; value3 $=\left(10 * \log 10\left(\operatorname{abs}\left(p p 12 \_3(i k,:)\right)\right)-10 * \log 10(\operatorname{abs}(p p 2(i k,:)))\right)$; value4 $=\left(10 * \log 10\left(\operatorname{abs}\left(p p 12 \_4(i k,:)\right)\right)-10 * \log 10(\operatorname{abs}(p p 2(i k,:)))\right)$;

value1L1=(10* $\left.\log 10\left(\operatorname{abs}\left(p p l 1 \_1(i k,:)\right)\right)-10 * \log 10(\operatorname{abs}(p p 1(i k,:)))\right)$; value2L1= $\left(10 * \log 10\left(\operatorname{abs}\left(p p l 1 \_2(i k,:)\right)\right)-10 * \log 10(\operatorname{abs}(\operatorname{pp} 1(i k,:)))\right)$; value3L1 $=\left(10^{*} \log 10\left(\operatorname{abs}\left(\operatorname{ppl1} \_3(i k,:)\right)\right)-10^{*} \log 10(\operatorname{abs}(\operatorname{pp} 1(i k,:)))\right)$; value4L1 $=\left(10 * \log 10\left(\operatorname{abs}\left(\operatorname{ppl1} \_4(i k,:)\right)\right)-10 * \log 10(\operatorname{abs}(\operatorname{pp} 1(i k,:)))\right)$;

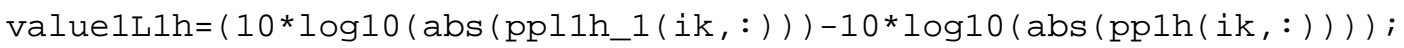
value2L1h $=\left(10^{*} \log 10\left(\operatorname{abs}\left(\operatorname{ppl1h\_ 2}(i k,:)\right)\right)-10^{*} \log 10(\operatorname{abs}(\operatorname{pp} 1 \mathrm{~h}(i k,:)))\right)$; value3L1h $=(10 * \log 10(\operatorname{abs}(\operatorname{ppl1h} 3(i k,:)))-10 * \log 10(\operatorname{abs}(\operatorname{pp} 1 \mathrm{~h}(i k,:))))$; value4L1h $=\left(10^{*} \log 10\left(\operatorname{abs}\left(\operatorname{ppl1h\_ 4}(i k,:)\right)\right)-10^{*} \log 10(\operatorname{abs}(\operatorname{pp} 1 \mathrm{~h}(i k,:)))\right)$;

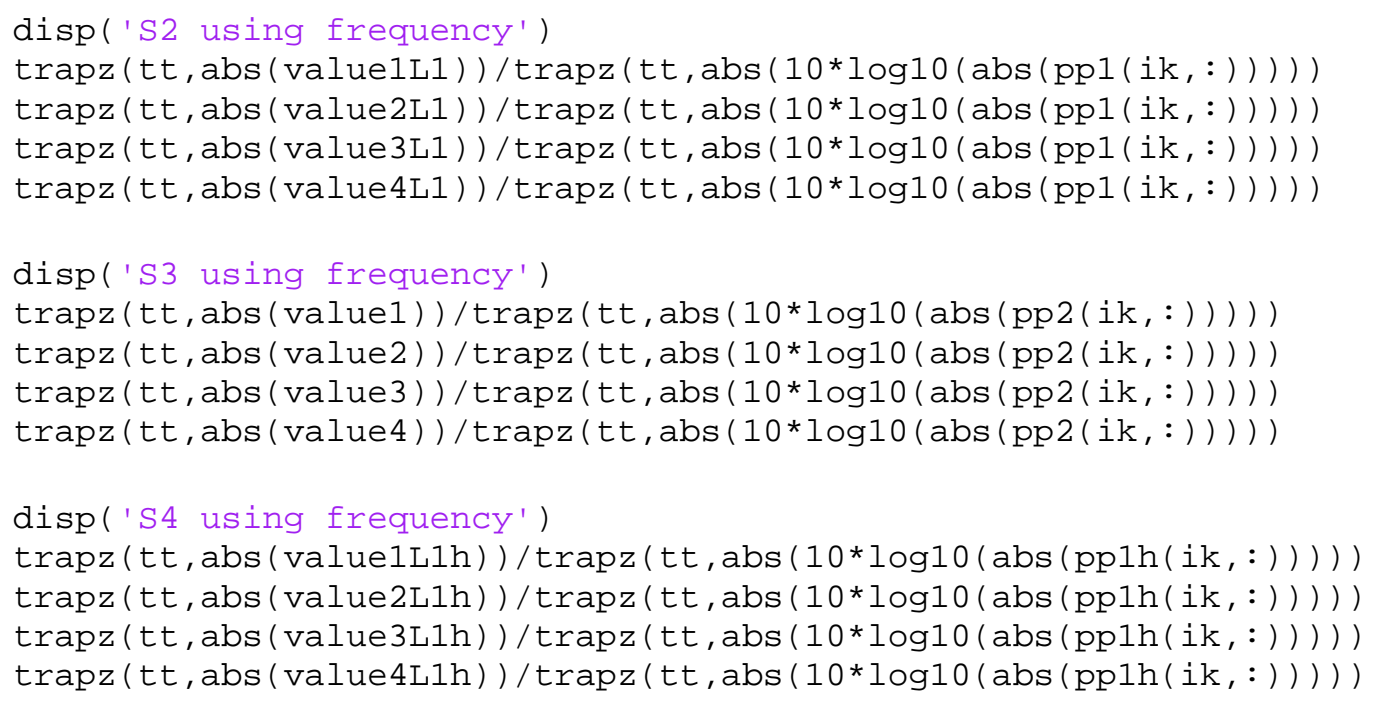



$\odot .4526$
0.507
๑. 3236
๑. 2801
0.3152
0.3565 ;
0.7272
$0.7191 \quad 0.4819$
$\odot .4401$
0.4241
○. 4858];

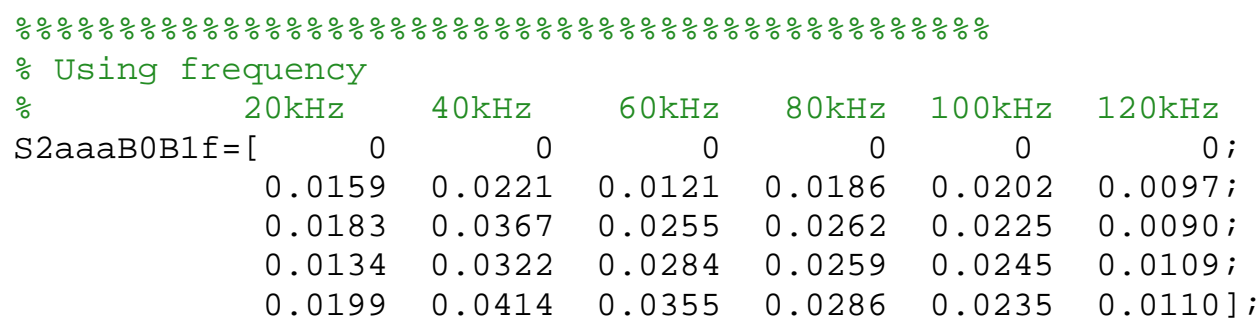

\begin{tabular}{|c|c|c|c|c|c|c|}
\hline S3aaaBOB $1 \mathrm{f}=$ & $\Theta$ & $\odot$ & 0 & 0 & $\odot$ & $0 ;$ \\
\hline & $\odot .0272$ & 0.0394 & 0.0321 & 0.0388 & 0.0326 & 0.0250 \\
\hline & 0.0546 & 0.056 & ๑.0394 & 0.0303 & 0.0424 & ๑. 0288; \\
\hline & 0.0523 & ๑. 0562 & 0.0433 & ๑. 0404 & $\odot .047$ & ๑.0338; \\
\hline & $\odot .0688$ & $\odot .0811$ & $\odot .0716$ & $\odot .0568$ & $\odot .0528$ & ๑. 0541] \\
\hline
\end{tabular}

\begin{tabular}{|c|c|c|c|c|c|c|}
\hline S4aaaBOB $1 \mathrm{f}=$ & 0 & 0 & 0 & 0 & $\odot$ & $\theta$ \\
\hline & $\odot .0242$ & 0.0393 & 0.0278 & $\odot .028$ & $\odot .0309$ & 0.0212 ; \\
\hline & 0.0415 & 0.0528 & 0.041 & 0.0364 & 0.0346 & ๑.0247; \\
\hline & 0.0535 & 0.0481 & 0.0443 & 0.0413 & 0.0383 & ๑.0265; \\
\hline & 0.062 & 0.0744 & 0.0496 & 0.0502 & 0.0451 & $0.0402]$ \\
\hline
\end{tabular}

\% %\%\%\%\%\%\%\%\%\%\%\%\%\%\%\%\%\%\%\%\%\%\%\%\%\%\%\%\%\%\%\%\%\%\%\%\%\%\%\%\%\%

a_x $=\left[\begin{array}{lllll}0 & 25 & 50 & 75 & 100\end{array}\right]$;

a_y $=\left[\begin{array}{llllll}20 & 40 & 60 & 80 & 100 & 120\end{array}\right]$;

a_z1t=[S2aaaBOB1t $(:, 1)$ ' ; S2aaaBOB1t $(:, 2)$ '; S2aaaBOB1t $(:, 3)$ '; S2aaaBOB1t $(:$, $4)^{\prime}$; S2aaaBOB1t $(:, 5)$ '; S2aaaBOB1t $(:, 6)$ '] ; ;

a_z2t=[S3aaaBOB1t $(:, 1)$ ' ; S3aaaBOB1t $(:, 2)$ ' ; S3aaaBOB1t $(:, 3)$ ' ; S3aaaBOB1t $(:$, $4)^{\prime}$; S3aаaBOB1t $(:, 5)$ '; S3aаaBOB1t $(:, 6)$ '] ;

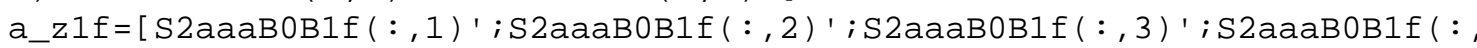
$4)^{\prime}$; S2aаaBOB1f $(:, 5)$ '; S2aaaBOB1f $(:, 6)$ ' $]$;

a_z2f=[S3aаaBOB1f $(:, 1)$ ' ; S3aаaBOB1f $(:, 2)$ ' ; S3aаaBOB1f $(:, 3)$ ' ; S3aаaBOB1f $(:$, $4)^{\prime}$; S3aаaBOB1f $(:, 5)$ '; S3aаaBOB1f $(:, 6)$ ' ] ;

a_z1th=[S4aaaBOB1t $(:, 1)$ ' ;S4aaaBOB1t $(:, 2)$ ' ;S4aaaBOB1t $(:, 3)$ ' ; S4aaaBOB1t ( : ,4) ';S4aaaBoB1t $(:, 5)$ '; S4aaaBOB1t $(:, 6)$ '] ;

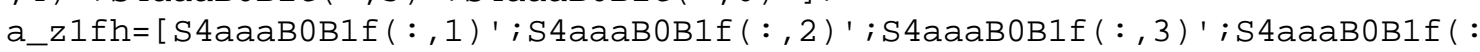
,4) ';S4aaaBOB1f $(:, 5)$ ';S4aaaBOB1f $(:, 6)$ '] ;

figure(31) \%DI_t at S2

set(gca, 'FontSize', 22, ' linewidth', 3, 'fontweight' ', 'demi')

$\mathrm{h}=\operatorname{surf}\left(\mathrm{a} \_\mathrm{x}, \mathrm{a} \_\mathrm{y}, \mathrm{a} \_z 1 \mathrm{t}\right)$;

set(h, 'Marker', 'o', 'MarkerSize' , 15, 'Linewidth ' ,1, 'MarkerFaceColor ', 'aut o')

$\operatorname{axis}\left(\left[\begin{array}{llllll}\odot & 100 & 20 & 120 & 0 & 1\end{array}\right]\right)$

set ( $h$, 'MarkerSize' , 15, 'Linewidth' , 4)

xlabel('Torque loss (\%)')

ylabel('Frequency $\left.(\mathrm{kHz})^{\prime}\right)$

zlabel('Damage Index')

figure(32) \%DI_t at S3

set(gca, 'FontSize', 22, 'linewidth', 3, 'fontweight', 'demi') 


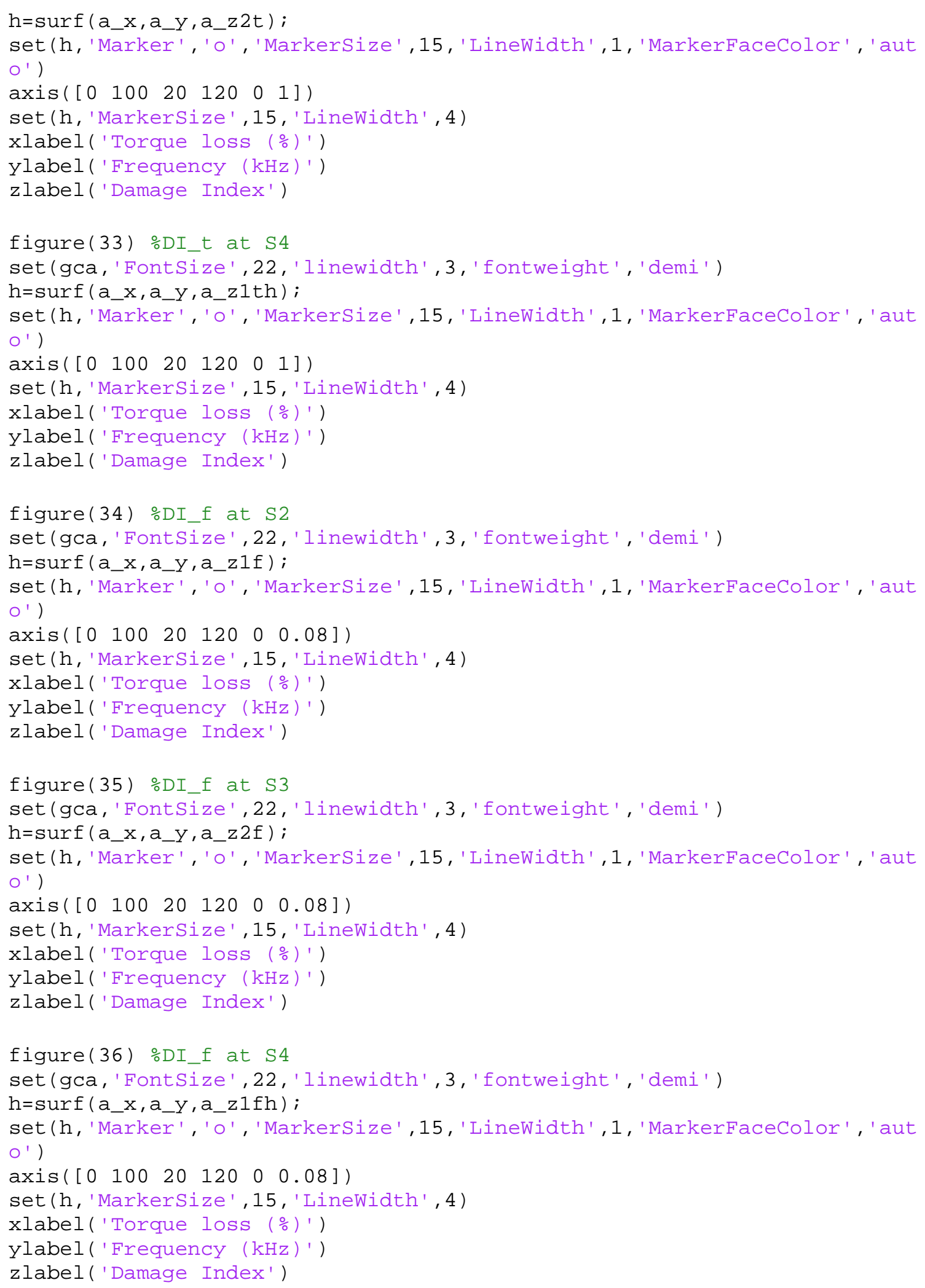




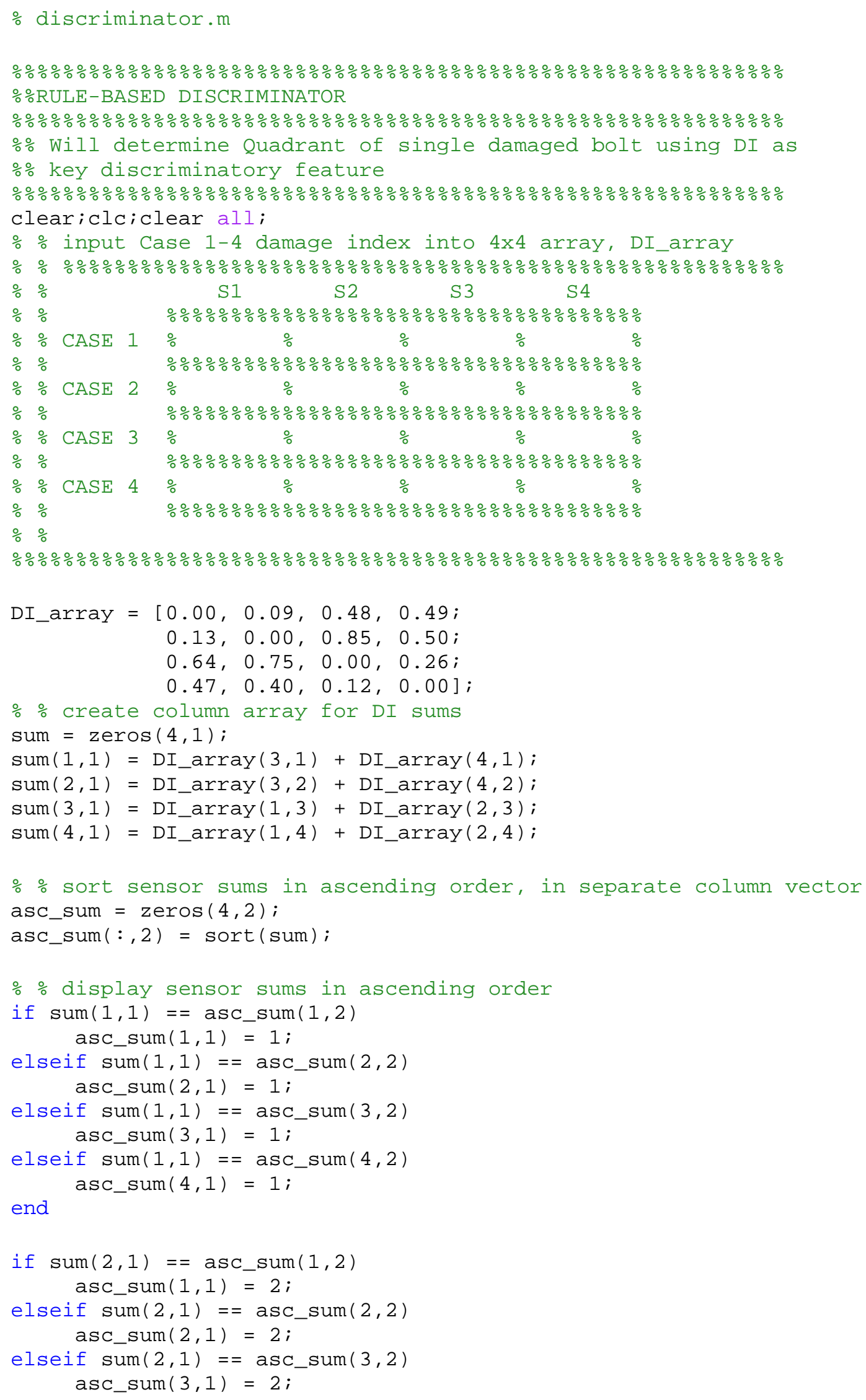




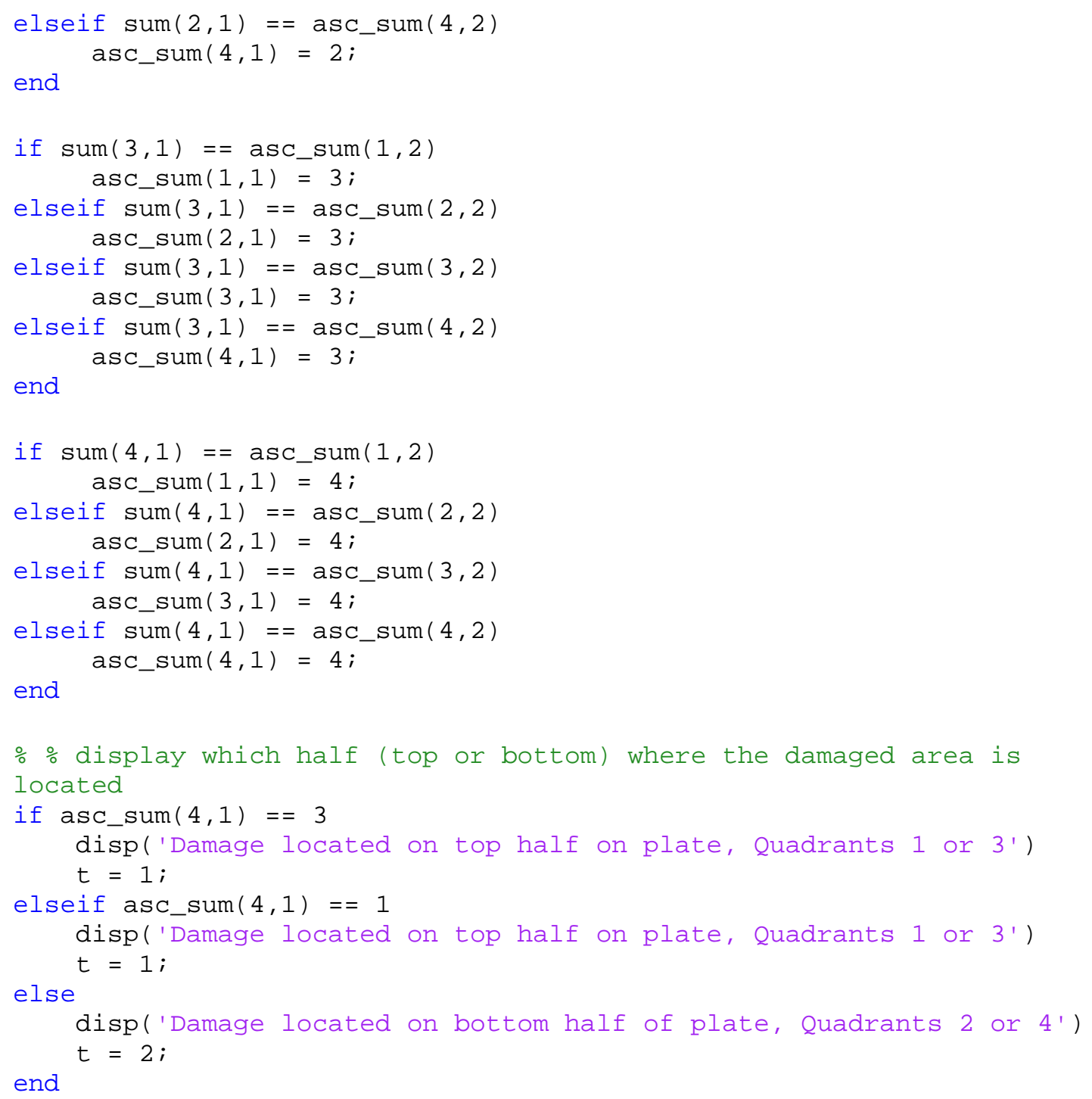




\section{Bibliography}

[1] "Columbia accident investigation report," Tech. Rep. Volume 1, Columbia Accident Investigation Board, 2003.

[2] D. Hunt, S. Weiss, W. West, T. Dunlap, and S. Freemeyer, "Development and implementation of a shuttle modal inspection system," Sound and Vibration, 1990.

[3] Y. Tong, "Literature review on aircraft structural risk and reliability analysis," Tech. Rep. DSTO-TR-1110, DSTO Aeronautical and Maritime Research Laboratory, 2001.

[4] J. Lincoln and R. Melliere, "Economic Life Determination for a Military Aircraft," Journal of Aircraft 36(5), pp. 737-742, 1999.

[5] R. Gopalan and K. Talluri, "The aircraft maintenance routing problem," Operations Research 46(2), pp. 260-271, 1998.

[6] U. Kumar, "New trends in aircraft reliability and maintenance measures," Journal of Quality in Maintenance Engineering 5(4), pp. 287-295, 1999.

[7] W. Marx, D. Mavris, and D. Schrage, "A knowledge-based system integrated with numerical analysis tools for aircraft life-cycle design," Cambridge University Press: Artificial Intelligence for Engineering Design, Analysis and Manufacturing 12, pp. 211-229, 1998.

[8] C. Farrar, D. Nix, T. Duffey, P. Cornwall, and G. Pardoen, "Damage Identification with Linear Discriminant Operators," in Proceedings of the $17^{\text {th }}$ Annual International Modal Analysis Conference, vol. 3727(2), pp. 599-607, 1999. 
[9] A. Rytter, Vibration Based Inspection of Civil Engineering Structures. PhD dissertation, Aalborg University, Department of Building Technology and Structural Engineering, 1993.

[10] S. Doebling, C. Farrar, M. Prime, and D. Shevitz, "Damage identification and health monitoring of structural and mechanical systems from changes in their vibration characteristics: A literature review," Tech. Mem. LA-13070-MS, Los Alamos National Laboratories, 1996.

[11] H. Sohn, C. Farrar, F. Hemez, D. Shunk, D. Stinemates, and B. Madler, "A review of structural health monitoring literature,” Tech. Mem. LA-13976-MS, Los Alamos National Laboratories, 2003.

[12] C. Farrar, S. Doebling, P. Cornwall, and E. Straser, "Variability of modal parameters measured on the Alamosa Canyon Bridge," in Proceedings on the $15^{\text {th }}$ International Modal Analysis Conference, vol. 3089(1), pp. 257-263, 1997.

[13] S. Doebling, C. Farrar, and R. Goodman, "Effects of measurement statistics on the detection of damage in the Alamosa Canyon Bridge," in Proceedings on the $15^{\text {th }}$ International Modal Analysis Conference, vol. 3089(1), pp. 919-929, 1997.

[14] S. Smith and P. McGowan, "Locating damaged members in a truss structure using modal test data: A demonstration experiment," in Proceedings of the $36^{\text {th }}$ AIAA/ASME/ASCE/AHS/ASC Structures, Structural Dynamics and Materials Conference, No. AIAA-89-1291-CP, 1989.

[15] A. Rytter and P. Kirkegaard, "Vibration based inspection using neural networks," in Proceedings of DAMAS 1997, Structural Damage Assessment Using Advanced Signal Processing Procedures, pp. 97-108, 1997. 
[16] O. Loland and J. Dodds, "Experience in developing and operating integrity monitoring systems in north sea," in Proceedings of the $8^{\text {th }}$ Annual Offshore Technology Conference, pp. 313-319, 1976.

[17] S. Kessler, Piezoelectric-Based In-Situ Damage Detection of Composite Materials for Structural Health Monitoring Systems. PhD dissertation, Massachusetts Institute of Technology, Department of Aeronautics and Astronautics, 2002.

[18] J. Tracy and G. Pardeon, "Effect of delamination on the natural frequencies of composite laminates," Journal of Composite Materials 23, pp. 1200-1215, 1989.

[19] D. Sanders, Y. Kim, and N. Stubbs, "Non-destructive evaluation of damage in composite structures using modal parameters," Experimental Mechanics 32(3), pp. 240-251, 1992.

[20] A. Purekar, Piezoelectric Phased Array Acousto-Ultrasonic Interrogation of Damage in Thin Plates. PhD dissertation, University of Maryland, Department of Aerospace Engineering, 2006.

[21] D. Mahapatra and S. Gopalakrishnan, "Spectral finite element analysis of coupled wave propagation in composite beams with multiple delaminations and strip inclusions," International Journal of Solids and Structures 41, pp.1173-1208, 2004.

[22] Y-T. Choi, "Wireless, self-powered, smart sensor system," Interim Report, UMd Acousto-Ultrasonics Task, University of Maryland, 2007.

[23] N. Stubbs and R. Osegueda, "Global non-destructive damage evaluation in solids," Modal Analysis: The International Journal of Analytical and Experimental Modal Analysis 5(2), pp. 67-79, 1990. 
[24] N. Stubbs and J. Kim, "Damage localization in structures without baseline modal parameters," AIAA Journal 34(8), pp. 1644-1649, 1996.

[25] A. Sierra, "Evolution of Fisher discriminants," IEEE Journal 3(1), pp. 747-752, 2001.

[26] R. Fisher, "The use of multiple measurements in taxanomic problems," Annals of Eugenics, Cambridge University Press 7, pp. 179-188, 1936.

[27] S. Raudys and A. Jain, "Small sample size effects in statistical pattern recognition: Recommendations for practitioners," IEEE Transactions on Pattern Analysis and Machine Intelligence 13(3), pp. 252-264, 1991.

[28] J. Galan and R. Abascal, "Numerical simulation of Lamb wave scattering in semiinfinite plates," International Journal for Numerical Methods in Engineering 53, pp. $1145-1173,2002$.

[29] P. Wilcox, M. Lowe, and P. Cawley, "Mode and transducer selection for long-range Lamb inspection,” Journal of Intelligent Material Systems and Structures 12(8), pp. $553-565,2001$.

[30] J. Yang and F. Chang, "Detection of bolt loosening in C-C composite thermal protection panels," Journal of Smart Materials and Structures 15, pp. 591-599, 2006.

[31] H. Wang, C. Hung, and F. Chang, "Bearing failure of bolted composite joints," Journal of Composite Materials 30(12), pp. 128-1313, 1996.

[32] N. Clark and S. Thwaites, "Local phase velocity measurements in plates," Journal of Sound and Vibration 187(2), pp. 241-252, 1995. 
[33] K. Tan, N. Guo, B. Wong and C. Tui, "Experimental evaluation of delaminations in composite plates by the use of Lamb waves," Composite Science and Technology 53, pp. 77-84, 1995.

[34] G. Yen and K. Lin, "Conditional health monitoring using vibration signatures," in Proceedings of the $38^{\text {th }}$ Conference on Decision and Control, pp. 4493-4498, 1999.

[35] R. Ma and H. Fu, "Smart active sensing technique using wavelet analysis method on damage detection of a composite plate," in Proceedings of the 2004 IEEE International Conference on Networking, Sensing and Control, pp. 773-777, 2004.

[36] N. Guo and P. Cawley, "The interaction of Lamb waves with delaminations in composite laminates," Journal of Acoustic Soc. Am. 94(4), pp. 2240-2246, 1993. 